\title{
Wihstutz, Anne [Hrsg.] \\ Zwischen Sandkasten und Abschiebung. Zum Alltag junger Kinder in Unterkünften für Geflüchtete
}

Opladen; Berlin; Toronto : Verlag Barbara Budrich 2019, 240 S.

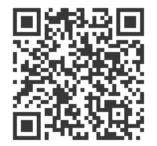

Quellenangabe/ Reference:

Wihstutz, Anne [Hrsg.]: Zwischen Sandkasten und Abschiebung. Zum Alltag junger Kinder in Unterkünften für Geflüchtete. Opladen; Berlin; Toronto : Verlag Barbara Budrich 2019, 240 S. URN: urn:nbn:de:0111-pedocs-182467 - DOI: 10.25656/01:18246

https://nbn-resolving.org/urn:nbn:de:0111-pedocs-182467

https://doi.org/10.25656/01:18246

in Kooperation mit / in cooperation with:

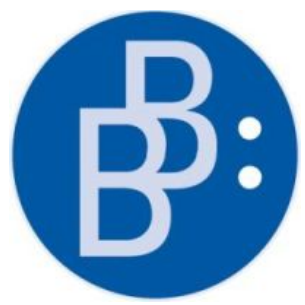

https://www.budrich.de

\section{Nutzungsbedingungen}

Dieses Dokument steht unter folgender Creative Commons-Lizenz: http://creativecommons.org/licenses/by-sa/4.0/deed.de - Sie dürfen das Werk bzw. den Inhalt vervielfältigen, verbreiten und öffentlich zugänglich machen sowie Abwandlungen und Bearbeitungen des Werkes bzw. Inhaltes anfertigen, solange sie den Namen des Autors/Rechteinhabers in der von ihm festgelegten Weise nennen und die daraufhin neu entstandenen Werke bzw. Inhalte nur unter Verwendung von Lizenzbedingungen weitergeben, die mit denen dieses Lizenzvertrags identisch, vergleichbar oder kompatibel sind.

Mit der Verwendung dieses Dokuments erkennen Sie die Nutzungsbedingungen an.

\section{Terms of use}

This document is published under following Creative Commons-License: http://creativecommons.org/licenses/by-sa/4.0/deed.en - You may copy, distribute and transmit, adapt or exhibit the work or its contents in public and alter, transform, or change this work as long as you attribute the work in the manner specified by the author or licensor. New resulting works or contents must be distributed pursuant to this license or an identical or comparable license.

By using this particular document, you accept the above-stated conditions of use.

\section{Kontakt / Contact:}

\section{peDOCS}

DIPF | Leibniz-Institut für Bildungsforschung und Bildungsinformation Informationszentrum (IZ) Bildung

E-Mail: pedocs@dipf.de

Internet: www.pedocs.de

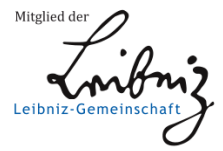


Zwischen Sandkasten und Abschiebung 
Anne Wihstutz (Hrsg.)

Zwischen Sandkasten und Abschiebung

Zum Alltag junger Kinder in Unterkünften für Geflüchtete

Verlag Barbara Budrich

Opladen • Berlin • Toronto 2019 
Bibliografische Information der Deutschen Nationalbibliothek

Die Deutsche Nationalbibliothek verzeichnet diese Publikation in der Deutschen Nationalbibliografie; detaillierte bibliografische Daten sind im Internet über http://dnb.d-nb.de abrufbar.

\section{Finanziert mit freundlicher Unterstützung der Bertelsmann Stiftung}

(C) 2019 Dieses Werk ist beim Verlag Barbara Budrich erschienen und steht unter der Creative Commons Lizenz Attribution-ShareAlike 4.0 International (CC BY-SA 4.0): https://creativecommons.org/licenses/by-sa/4.0/.

Diese Lizenz erlaubt die Verbreitung, Speicherung, Vervielfältigung und Bearbeitung bei Verwendung der gleichen CC-BY-SA 4.0-Lizenz und unter Angabe der UrheberInnen, Rechte, Änderungen und verwendeten Lizenz. www.budrich.de

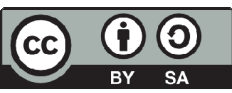

Dieses Buch steht im Open-Access-Bereich der Verlagsseite zum kostenlosen Download bereit (https://doi.org/10.3224/84742222)

Eine kostenpflichtige Druckversion (Print on Demand) kann über den Verlag bezogen werden. Die Seitenzahlen in der Druck- und Onlineversion sind identisch.

$\begin{array}{ll}\text { ISBN } & 978-3-8474-2222-8 \text { (Paperback) } \\ \text { eISBN } & 978-3-8474-1250-2 \text { (eBook) } \\ \text { DOI } & \text { https://doi.org/10.3224/84742222 }\end{array}$

Druck: paper \& tinta, Warschau

Umschlaggestaltung: Bettina Lehfeldt, Kleinmachnow - www.lehfeldtgraphic.de

Titelbildnachweis: Michaela Schultz

Lektorat und Satz: Anja Borkam, Jena - kontakt@lektorat-borkam.de

Lektorat der englischsprachigen Texte: Máiréad Collins, Belfast, UK 


\section{Vorwort}

Unter den nach Deutschland geflüchteten und asylsuchenden Menschen befinden sich viele Kinder. Die UN-Kinderrechtskonvention verpflichtet die deutschen Behörden, diesen Kindern besonderen Schutz und ein menschenwürdiges Leben zu gewährleisten. Das UN-Hochkommissariat für Flüchtlinge, das Deutsche Institut für Menschenrechte und andere Menschenrechtsorganisationen haben immer wieder darauf hingewiesen, dass die Bundesrepublik Deutschland ihren Verpflichtungen trotz mancher Verbesserungen noch immer nicht nachkommt. In verschiedenen Berichten wird dokumentiert, dass geflüchtete Kinder ebenso wie Erwachsene als „Fremde“ rassistischer Diskriminierung und Gewalt ausgesetzt sind, dass sie keine angemessenen Bildungsmöglichkeiten vorfinden und insbesondere in Massenunterkünften von einem kindgerechten sozialen Leben abgeschnitten und in ihren Entwicklungsmöglichkeiten eingeschränkt sind. Doch kaum ist bisher untersucht worden, wie Kinder, die nach einer häufig dramatischen und mit traumatischen Erlebnissen verbundenen Flucht in Deutschland ankommen, selbst ihre Situation erleben und sich darin zurechtfinden. Dies gilt besonders für junge Kinder.

In dem Buch „Zwischen Sandkasten und Abschiebung - Zum Alltag junger Kinder in Unterkünften für Geflüchtete" wird erstmals ein tiefergehender Einblick in den Alltag und das Erleben von geflüchteten Kindern vermittelt. Im Zentrum stehen junge, noch nicht schulpflichtige Kinder, die in sogenannten Erstaufnahmeeinrichtungen bzw. Gemeinschaftsunterkünften untergebracht sind. In einem Forschungsprojekt des Instituts für Innovation und Beratung, INIB e. V., an der Evangelischen Hochschule Berlin und des Instituts für den Situationsansatz an der Internationalen Akademie Berlin haben Sozialwissenschaftlerinnen acht Monate lang Kinder in drei Berliner Sammelunterkünften für geflüchtete und migrierte Menschen begleitet, in denen sie mit ihren Angehörigen leben. Die Autorinnen verstehen ihre ethnografische Studie als ,engagierte Forschung“, die dazu beitragen soll, den Alltag der Kinder aus deren eigenen Perspektive zu verstehen und ihre Situation gemäß der ihnen zustehenden Kinderrechte grundlegend zu verbessern.

Ohne die soziale Marginalisierung, die Verletzlichkeit und die Diskriminierungs- und Gewalterfahrungen der Kinder kleinzureden, kommen diese in den ,dichten Beschreibungen“ des Buches als soziale Subjekte zum Vorschein, die eigene Sichtweisen haben und sich bei verschiedenen Gelegenheiten aktiv mit ihrer prekären Situation auseinandersetzen. Das ethnografische Herangehen und die bei der teilnehmenden Beobachtung praktizierte Empathie der Forscherinnen ermöglicht, die Eigensinnigkeit und das Handlungspotential der Kinder auch aus kleinen Episoden herauszulesen. Das Handeln der Kinder und seine Reichweite werden dabei nicht idealisiert, sondern mit den häufig ein- 
schränkenden und sogar demütigenden Lebensbedingungen in einer relativ isolierten, lagerähnlichen Situation in Beziehung gesetzt; all dies zusätzlich überschattet von bleiberechtlicher Unsicherheit und drohender Abschiebung.

Die Betrachtung der Lebenssituation der Kinder und ihrer Erfahrungen lässt sich nicht ablösen von dem Umstand, dass die in den letzten Jahren gewachsene Zahl der Menschen, die in Deutschland Schutz suchen, mit sich zuspitzenden politischen Kontroversen einhergeht. Ihre Anwesenheit wurde zu einer „Flüchtlingskrise“ hochstilisiert, und die nach Deutschland geflüchteten Menschen werden von Teilen der einheimischen Bevölkerung, angeheizt von rechtspopulistischer Demagogie, zum Sündenbock für eigene Probleme gemacht. Die Absonderung in Massenunterkünften leistet dem Vorschub, da sie die Einheimischen und Geflüchteten spaltet und ein alltägliches Miteinander praktisch unmöglich macht. Auch eine sich als solidarisch und partizipativ verstehende Sozialarbeit stößt unter solchen institutionellen Bedingungen an enge Grenzen.

Gerade mit Blick auf das Erleben und die Identitätsbildung der Kinder ist deshalb migrationspolitisch darauf zu bestehen, dass geflüchtete Menschen ein gleichberechtigtes Leben inmitten der Bevölkerung führen können. Für junge Kinder kommt dabei dem Recht auf frühkindliche Bildung und das Zusammensein mit Kindern verschiedener Herkunft in Kindertageseinrichtungen besondere Bedeutung zu, was allerdings auch erforderlich macht, dass das pädagogische Personal migrationspädagogisch kompetent agiert und geflüchtete Kinder vorbehaltslos unterstützt und fördert. Menschen „,mit Migrationshintergrund" sind zumindest im städtischen Umfeld in Deutschland längst selbstverständlicher Teil des Alltags geworden, und immer mehr Menschen blicken auf transnationale Biografien zurück. Selbst wenn die geflüchteten Menschen in ihre Herkunftsorte zurückkehren wollen und sich eines Tages hierfür eine Möglichkeit ergibt, werden ihre Kinder von den Erfahrungen, die sie in Deutschland machen, beeinflusst sein und vor der Frage stehen, wo und wie sie weiter leben wollen. Die geflüchteten Kinder können damit richtungsweisend transnationale Räume sowie inter- und transkultureller Begegnungen gestalten und dazu beitragen, dass engstirnige Verfechter*innen einer sich als „rein deutsch“ verstehenden homogenen Nationalkultur auf verlorenem Posten stehen.

Das Buch richtet sich gegen das, was die Autorinnen ,doppelte Viktimisierung" nennen. Eine solche sehen sie dann gegeben, wenn die Kinder unter Verweis auf ihre Leidensgeschichte nur als hilflose Opfer gesehen und Maßnahmen unterworfen werden, die zwar ihrem Schutz dienen sollen, sie aber aufs Neue zu Objekten fremdbestimmter Entscheidungen herabwürdigen. Die Autorinnen sehen ausdrücklich in Verletzlichkeit und Handlungsfähigkeit keinen Widerspruch und plädieren für ein Verständnis von Schutz, das die Kinder als handlungsfähige Akteure ernst nimmt und ihnen die Möglichkeit gibt, an allen sie betreffenden Entscheidungen mitzuwirken. Ein solches Verständnis ent- 
spricht dem Gedanken der Unteilbarkeit von Schutz-, Förder- und Partizipationsrechten, wie er in der UN-Kinderrechtskonvention verankert ist.

Die in dem Buch versammelten Beiträge beleuchten neben ethischen und methodologischen Fragen des Forschungsprojekts unterschiedliche Aspekte der Situation geflüchteter Kinder. Nach Reflexionen zum widersprüchlichen Verhältnis von Kinderrechten und Asylpolitik wird sichtbar gemacht, wie sich Kinder in der Gemeinschaftsunterkunft in „kleinen Revolutionen“ eigene Spiel- und Handlungsräume erobern, aber auch, wie sie Gewalt erleben und sich vor ihr zu schützen versuchen. Die einfühlsamen und eindrucksvollen Beschreibungen werden mit migrationspolitischen und kindheitstheoretischen Überlegungen verbunden und geben wichtige Hinweise, wie Kinder unter den Bedingungen, die in Sammelunterkünften herrschen, solidarisch begleitet und ermutigt werden können. Sie machen aber auch deutlich, dass das Leben in diesen Unterkünften nicht länger hinzunehmen ist. Der Band schließt mit sehr bedenkenswerten kinderpolitischen und pädagogischen Empfehlungen, die politischen Entscheidungsträger*innen und Fachkräften in der Betreuung geflüchteter und asylsuchender Kinder neue Orientierungen vermitteln.

Den Forscherinnen ist durchaus bewusst, dass ihr Verhältnis zu den Kindern selbst von ungleicher Macht gekennzeichnet ist und deshalb permanente kritische Selbstreflexion über die eigene Rolle erforderlich ist. Sie sehen sich auch herausgefordert, ihre Forschung daraufhin zu hinterfragen, ob sie tatsächlich den Kindern selbst zugutekommt, und sich mit den Kindern über den Sinn ihrer Forschung zu verständigen. Nach meinem Eindruck ist ein Buch entstanden, in dem die Sichtweisen, Gedanken, Sorgen und Wünsche geflüchteter, in Sammelunterkünften lebender Kinder auf lebendige und nachvollziehbare Weise zum Ausdruck kommen. Ich wünsche ihm viele aufmerksame Leser*innen.

Manfred Liebel 



\section{Inhalt}

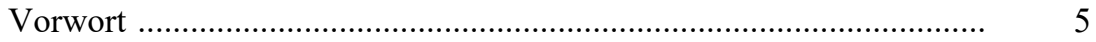

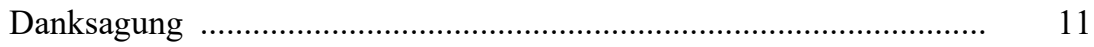

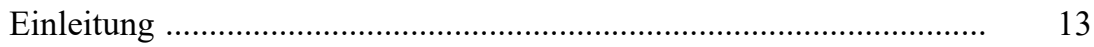

Anne Wihstutz

Das Forschungsprojekt und sein Design .......................................... 25

Anne Wihstutz

Mittendrin und außen vor - Geflüchtete Kinder und die Umsetzung

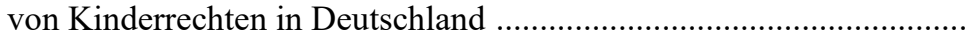

Hoa Mai Trần

Ethisch-reflexive Auseinandersetzungen im Forschungsprozess

Sarah Fichtner \& Hoa Mai Trần

Handlungs-Spiel-Räume von Kindern in

Gemeinschaftsunterkünften

Penelope Scott \& Thi Huyen Trang Le

"It's child's play - or is it?" Play, conflicts and relationship networks among children in a reception centre

Evelyn Schulz-Algie

„MANNO STOPP!“ Das Menschenrecht von jungen Kindern auf

Schutz vor Gewalt in Unterkünften für geflüchtete Menschen

Penelope Scott

Entering the field as researchers and leaving as "aunties":

Field relations with young children and their families in a refugee reception centre

\section{Anne Wihstutz}

Zentrale Erkenntnisse und Empfehlungen 



\section{Danksagung}

An erster Stelle gilt unser Dank den Kindern, Eltern und Mitarbeiter*innen aus den Unterkünften für Geflüchtete für ihre Bereitschaft, sich an der Forschung zu beteiligen. Erst durch sie wurde das Projekt möglich. Auch gilt der Stiftung Deutsche Jugendmarke, dem Paritätischen Landesverband Berlin, der Stiftung Auxilium sowie der Bertelsmann Stiftung Dankbarkeit für die finanzielle Förderung des Forschungsprojekts und der Publikation. Großer Dank gilt dem Berliner Bündnis für Kinder geflüchteter Familien, Willkommen KONKRET für die Impulsgebung sowie für das Vertrauen in die Bearbeitung der Forschungsfrage. Für ihre konstruktiv-kritische Begleitung des Forschungsprozesses als „,critical friends“ gebührt den Kolleginnen der Alice-Salomon Hochschule Berlin, der FH Potsdam, des Berliner Institut für empirische Integrations- und Migrationsforschung (BIM) an der HU Berlin, der Universität Vechta sowie der Hochschule Magdeburg-Stendal unser Dank. Danke auch für die Peer-Reviews und Anmerkungen zu früheren Versionen der Buchbeiträge. Für ihre anregenden Kommentare zum Datenerhebungs- und -interpretationsprozess sei an dieser Stelle auch den Teilnehmer*innen der Forschungswerkstatt des Instituts für Innovation und Beratung (INIB e. V.) und den Supervisorinnen für die Unterstützung während des Forschungsprozesses gedankt. Ein großes Dankeschön gilt zudem all den Kolleg*innen im Institut für den Situationsansatz (ISTA) und im INIB e. V., die hinter den Kulissen den Forschungsund Publikationsprozess sachkundig begleitet haben. Auch der Lektorin und der Designerin des Buchcovers sei an dieser Stelle für ihre kreative Arbeit gedankt. Ganz besonderer Dank gilt jedoch den Projektmitarbeiterinnen Seyran Bostanc1, Dr. Sarah Fichtner, Thi Huyen Trang Le, Hannah Rosenfeld, Evelyn Schulz-Algie, Dr. Penelope Scott, Sonja Stegmaier, Hoa Mai Trần und Clara Zimmer für ihr außerordentliches Engagement sowie auch Petra Wagner vom ISTA für die Koleitung des Projekts. Schließlich danken wir auch Sarah Rögl vom Barbara Budrich Verlag für das entgegengebrachte Vertrauen, ihre Geduld und Unterstützung bei der Veröffentlichung dieser Studie.

Mai 2019

Anne Wihstutz für das Projektteam 



\section{Einleitung}

„The direct experience of conflict-induced migration constitutes the very antithesis of the ideal of childhood as a period of safety and continuity, free of onerous responsibility that has long formed the bedrock of European-American thinking“ (Boyden \& Hart 2007: 246).

\section{Ausgangslage}

In jüngster Zeit erlebt die Welt Flucht- und Migrationsbewegungen, die es in diesem Ausmaß seit dem Zweiten Weltkrieg nicht mehr gegeben hat. Ende 2017 sahen sich weltweit insgesamt 68,5 Millionen Menschen gezwungen zu migrieren. 84 Prozent der Flüchtlinge leben laut dem Hohen Flüchtlingskommissariat der Vereinten Nationen (UNHCR) in Entwicklungsländern (vgl. Sachverständigenrat deutscher Stiftungen für Integration und Migration, SVR 2018). Im Zusammenhang mit dem Bürgerkrieg in Syrien und den anhaltenden Kämpfen in Afghanistan, Irak und Südsudan stieg seit 2010 die Zahl der international Schutzsuchenden (vgl. UNHCR 2017). Die große Mehrheit von 40,3 Millionen flieht als Binnenflüchtlinge innerhalb des eigenen Landes oder in Nachbarländer (vgl. SVR 2017). Von den 25,4 Millionen Flüchtlingen gemäß der Genfer Flüchtlingskonvention sind über 52 Prozent minderjährig (vgl. UNHCR 2018; Engler \& Krause 2018). Von den Flüchtenden weltweit gelangen nur wenige nach Europa, zumeist über das Mittelmeer und die sogenannte Balkanroute. Einen Höhepunkt erreichte die Zahl Schutzsuchender in Europa im Sommer 2015. Allein in Deutschland wurden 890.000 Asylsuchende im Jahr 2015 registriert (vgl. Bundesministerium des Inneren, BMI 2016).

Die europäischen Regierungen reagierten mit Maßnahmen, die die Einreise von Schutzsuchenden und ihre Bewegungsfreiheit in Europa drastisch einschränken sollten: ${ }^{1}$ Die faktische Schließung der Grenzen auf der sogenannten Balkanroute zum 9. März 2016 sowie vor allem das sogenannte Flüchtlings-

1 Eine Verschärfung asylrechtlicher Politik setzte nicht erst mit dem als ,langer Sommer der Migration“" benannten Entwicklungen im Jahr 2015 ein. Bereits seit den 1990er Jahren im Zusammenhang mit dem Zusammenbruch der Sowjetunion und dem damit verknüpften Anwachsen der Asylantragszahlen aus Ost-, Ostmittel- und Südosteuropa ist in Europa eine restriktive asylrechtliche Politik zu beobachten (vgl. Bade \& Oltmer 2005). In Deutschland wurde im Zuge einer politisch und medial geführten Debatte das Recht auf Asyl im Jahr 1993 derart stark eingegrenzt, dass es zu seiner faktischen Fastabschaffung geführt hat (vgl. Weinzierl 2010). 
abkommen zwischen der Europäischen Union und der Türkei am 18. März 2016 führten dazu, dass weniger Schutzsuchende einen EU-Mitgliedsstaat erreichten und Asyl beantragen konnten. Zur verstärkten Sicherung der EU-Außengrenzen wurden die europäische Grenzschutzagentur Frontex eingesetzt und Grenzkontrollen im Schengen-Raum eingeführt, um Schutzsuchende, die in einem Land der EU erstregistriert wurden, daran zu hindern, innerhalb der EU weiterzureisen. Infolge dieser Maßnahmen wurden im Jahr 2017 nur ungefähr halb so viele Asylerstanträge in Europa gestellt wie noch im Jahr zuvor. Laut dem Statistischen Amt der Europäischen Union (Eurostat) beantragten 2017 insgesamt knapp 650.000 Personen erstmalig Asyl in Europa (UNHCR 2018). ${ }^{2}$ In Deutschland wurden 198.300 Erstanträge auf Asyl gestellt, von diesen bezogen sich 6267 (3,2 Prozent) auf Kinder im Alter zwischen vier und sechs Jahren und 46.096 (23,2 Prozent) auf Kinder unter vier Jahren (Bundesamt für Migration und Flüchtlinge, BAMF 2018). Mit anderen Worten: Während im Jahr 2016 in Deutschland noch 9 Asylanträge auf 1000 Einwohner*innen kamen, waren es im Jahr 2017 nur noch 2 Asylanträge pro 1000 Einwohner*innen (vgl. SVR 2018: $9 \mathrm{f}$.); ein deutlicher Rückgang auch gegenüber 2015. Von den ca. 890.000 im Jahr 2015 registrierten Asylsuchenden in Deutschland war knapp die Hälfte (44 Prozent) minderjährig (SVR 2017: 2).

In den Jahren 2015/2016 sahen sich die zuständigen Behörden in Deutschland nicht in der Lage, adäquat zu handeln. Bundesweit kam es bei dem Versuch der Erstregistrierung von Schutzsuchenden zu chaotischen Zuständen. Auf Landes- und kommunaler Ebene stellte die Versorgung der Betroffenen eine große Herausforderung dar (Abgeordnetenhaus 2015). Die Institutionen standen den gestiegenen Anforderungen weitestgehend unvorbereitet gegenüber. Die Situation der Antragsteller*innen in Berlin war katastrophal. Das damals zuständige Landesamt für Gesundheit und Soziales (LaGeSo) konnte über Monate organisatorisch den Bedarfen nicht nachkommen. Hastig wurden Notquartiere aus dem Boden gestampft, Turnhallen kurzerhand in Notunterkünfte umfunktioniert, um Schutzsuchenden ein erstes Obdach zu geben, unter ihnen auch Familien mit Kindern.

Während Flucht und Vertreibung in Europa keine neuen Phänomene sind, ist aktuell eine Dynamik in den Aufnahmeländern zu beobachten, die Ankommende in ,,deserving' refugee“" und ,,undeserving " migrant" (Holmes \& Castañeda 2016: 12) bzw. „erwünschte und unerwünschte“ Gruppen Schutzsuchender teilt (Bundesfachverband unbegleiteter minderjähriger Flüchtlinge, BumF 2016: 4). Die Differenzierung orientiert sich unter anderem an den Herkunftsländern wie den sogenannten sicheren Herkunftsländern (vgl. ebd.). Im Zusammenhang mit den Terroranschlägen vom 11. September 2001 in den USA werden Menschen aus arabischen Ländern bzw. muslimischen Glaubens

2 Im Jahr 2016 wurden in der Europäischen Union ca. 1,2 Millionen Asylanträge gestellt (SVR 2017), ungefähr die Hälfte davon in Deutschland (722.370 registrierte Asylerstanträge, BAMF 2016). 
zunehmend als politische Bedrohung der nationalen Sicherheit verhandelt (vgl. Vitus \& Lidén 2010). Auch Geflüchtete werden in Zusammenhang mit Terrorismus gesetzt (vgl. Pries 2016), wie nach den Terroranschlägen in Paris im November 2015, den Übergriffen auf Frauen in der Silvesternacht 2015 in Köln und dem Terroranschlag auf den Weihnachtsmarkt im Dezember in Berlin 2016 geschehen. Von einem humanitär verhandelten Thema wird die Flüchtlingsthematik zunehmend sicherheitspolitisch diskutiert und mit Begriffen assoziiert, die eher an Naturkatastrophen erinnern oder Bilder von Krieg und Militär nahelegen wie „Flüchtlingsströme“, „Flüchtlingswellen“, „Flüchtlingsfluten“ oder gar „Flüchtlingsinvasionen“, die sich gen Deutschland und Europa bewegen würden (vgl. BumF 2016: 9). Auch werden Metaphern verwendet, die den „Asylmissbrauch“ durch Schutzsuchende nahelegen (Tworek 2018; Holmes \& Castañeda 2016) wie in der Wortschöpfung „Asyltourismus“ durch den bayrischen Ministerpräsidenten Markus Söder (kit-Agentur für verständliche Sprache, 2018). Darüber werden diskursiv Bedrohungsszenarien kreiert, die Ängste und Vorbehalte bei Angehörigen der Mehrheitsgesellschaft gegenüber Schutzsuchenden wachrufen und weitere Einschränkungen im Recht nach sich ziehen.

\section{Flüchtlingsdiskurse}

Mit der Bezeichnung ganzer Gruppen von Menschen als „Flüchtlinge“ wird ihnen ein Sonderstatus zugewiesen, der sie als von der bestehenden Norm abweichend markiert. Ihre Biografien, Interessen sowie ihre unterschiedlichen sozialen, kulturellen, wirtschaftlichen und politischen Hintergründe verschwinden mit dem vereinheitlichenden Begriff Flüchtling. Mit dieser Statuszuweisung verbunden sind soziale Ein-, Ab- und Ausgrenzungsprozesse, die eine ,verrechtlichte Andersstellung“ und daran gebunden eine legitime Ungleichbehandlung Geflüchteter gegenüber der Bevölkerung des Aufnahmelandes begründet und zu ,einer sozialen Trennung zwischen Wir und Anderen (den Fremden)“ führt (vgl. Krause 2016: o. S.). Flüchtlinge werden zu Objekten, über die entschieden wird; die als „Problem“ verwaltet werden. Von Flüchtlingen wird erwartet, dass sie in Vorleistung gehen, sich entsprechend „,integrieren“, um sich der (Leistungs-)Unterstützung durch die Mehrheitsgesellschaft würdig zu erweisen.

In Anbetracht der politischen Instrumentalisierung von Geflüchteten als „Bedrohung“" oder als „Opfer“" (s. u.) ist es ein Anliegen des vorliegenden Buches, die Menschen, die hinter einem solchen „Label“ homogenisierend und abwertend zusammengefasst werden, als handelnde Subjekte zu beschreiben. Folglich sprechen wir uns gegen die Verwendung des Flüchtlingsbegriffs aus 
und machen sprachlich deutlich, dass wir die Personen als eigenständig oder auch kollektiv handelnde Akteur*innen verstehen, die sich mit ihrer Umwelt auseinandersetzen, die versuchen, auf ihre Unterbringungsverhältnisse einzuwirken und vielfältige Wünsche, Erfahrungen und Kompetenzen haben.

In diesem Buch werden die Begriffe Asylsuchende, Schutzsuchende, Geflüchtete und Menschen mit Fluchterfahrung als Synonyme verwandt, unabhängig von den Fluchtgründen und dem tatsächlichen Status der Personen im Aufnahmeland oder ob sie formal als Flüchtlinge nach der Genfer Konvention anerkannt wurden. ${ }^{3}$

\section{Zur Situation junger geflüchteter Kinder und ihrer Familien im Zeitraum 2016-2017 in Deutschland}

Die Lebensverhältnisse von Kindern werden während des Ankommens bzw. während der Transitsituation und ihrer Unterbringung in Massenunterkünften als kindeswohlgefährdend beschrieben (vgl. BumF 2016: 9). „Fast 8000 minderjährige Flüchtlinge leben in Notunterkünften. Die Berliner Sozialverwaltung schafft es nicht, minderjährige Flüchtlinge kindgerecht unterzubringen. Es seien einfach zu viele“ (Jens Anker in: Berliner Morgenpost 17.1.2016).

Geflüchtete Kinder und ihre Eltern unterliegen der Asyl- und Ausländergesetzgebung und den jeweiligen Verordnungen. Damit sind in der Erstaufnahme, -registrierung und -unterbringung zunächst die Länder und nachfolgend die Kommunen für die Unterbringung und Versorgung Geflüchteter in Deutschland zuständig. In einem ersten Überblick der vorhandenen Forschung zum Thema junger Geflüchteter in Deutschland beschreibt das Deutsche Jugendinstitut (DJI), dass Kindern der Raum für Rückzug, Ruhe und Entfaltung von eigenen Interessen in den Unterkünften in der Regel fehle (vgl. Soyer 2014). Auch der Sachverständigenrat deutscher Stiftungen für Integration und Migration (SVR) stellt 2015 fest, dass für Kinder Möglichkeiten fehlten, sich zu bewegen, zu spielen oder sich zurückzuziehen, und der Lärmpegel eine besondere Belastung darstelle. Insbesondere sei fraglich, wie grundlegende Rechte der UN-Kinderrechtskonvention - die seit der Rücknahme des „Ausländervorbehalts" durch die Bundesregierung für alle Kinder und Jugendlichen

3 Der Begriff Flüchtling ist völkerrechtlich über das Abkommen über die Rechtstellung der Flüchtlinge von 1951 (die Genfer Flüchtlingskonvention) und das Protokoll von 1967 definiert. Flüchtling ist demzufolge eine Person, die ,aus der begründeten Furcht vor Verfolgung wegen ihrer Rasse, Religion, Nationalität, Zugehörigkeit zu einer bestimmten sozialen Gruppe oder wegen ihrer politischen Überzeugung“ in einem anderen als dem Herkunftsland nach Schutz sucht (vgl. Artikel 1a, 2 der Genfer Flüchtlingskonvention zitiert nach Krause 2016; vgl. SVR 2018: 1). 
in Deutschland gelten - in Notunterkünften wie Traglufthallen oder Containerdörfern gewährleistet würden (vgl. SVR 2015: 4). Beteiligungsrechte von Kindern werden verletzt durch ihre vielerorts festgestellte Ausgrenzung von lokalen und gesellschaftlichen Teilhabemöglichkeiten (vgl. BumF 2016). In seiner Überblickstudie (Fact-Finding) hebt der Bundesfachverband unbegleitete minderjährige Flüchtlinge e. V. hervor, dass Kinder insbesondere in Erstaufnahmeeinrichtungen und Notunterkünften in kindeswohlgefährdenden Strukturen leben (vgl. ebd.: 9). Die Berücksichtigung des Kindeswohls bzw. der „best interests of the child" ist nicht gegeben. Ebenso ist nicht bekannt, wie viele Kinder aus den Unterkünften Regelangebote und Dienste der Kinder- und Jugendhilfe in Anspruch nehmen (vgl. Peucker \& Seckinger 2014). Verlässliche Zahlen fehlen. In Berlin reicht das vorhandene Angebot der Kindertagesbetreuung insgesamt nicht für den tatsächlichen Bedarf der einheimischen Bevölkerung. Umso schwerer ist der Zugang zu frühkindlicher Bildung für geflüchtete Kinder. Wie es um ihren Gesundheitszustand steht, erfasst eine repräsentative Studie der TU München aus dem Jahr 2015. Nach dieser Studie leiden ca. 22 Prozent der aus Syrien kommenden Kinder und Jugendlichen unter einer Posttraumatischen Belastungsstörung (PTBS) und 16 Prozent unter Anpassungsstörungen. Körperliche Beschwerden wie Karies (63 Prozent) und Atemwegserkrankungen (ca. 25 Prozent) sind häufig. Von den Minderjährigen haben 11 Prozent einen Infekt oder parasitäre Erkrankungen. Jedes zehnte Kind muss akut behandelt werden (vgl. BumF 2016: 13). Doch asyl- und aufenthaltsrechtliche Bestimmungen reduzieren die gesundheitliche Versorgung von Kindern auf Akutbehandlungen.

Neben diesen, das Existenzminimum und die Gesundheit der Kinder bedrohenden Verhältnissen kommt für asylsuchende Kinder und ihre Familien noch die Unsicherheit hinzu, wie sich ihre unmittelbare Zukunft rechtlich gestaltet. Solange ihr Asylantrag nicht beschieden ist, können Familien keine Sicherheit darüber erlangen, ob und wie lange sie bleiben können. Verfahren ziehen sich über Monate hin (BumF 2016: 10).

Diese kurze Schilderung macht deutlich, dass die Verhältnisse nach der Flucht zur besonderen Vulnerabilität von jungen geflüchteten Kindern beitragen. Sie werden, ,in erster Linie als Anhang ihrer Eltern wahrgenommen und behandelt, nicht als eigenständige Persönlichkeiten und Träger eigener Rechte, mit ganz besonderen, kindspezifischen Bedürfnissen“" (Bertold 2014: 10).

\section{Forschung mit geflüchteten Kindern}

Forschung mit Kindern in derart ungeschützten Verhältnissen stellt eine besondere Herausforderung dar. Einige Forschungsergebnisse untermauern die 
besondere Verletzlichkeit geflüchteter Kinder (für einen Überblick in Deutschland vgl. BumF 2016; Klein 2015; DJI 2014). ${ }^{4}$ Dabei dominiert eine erwachsenen-zentrierte Perspektive, die Kinder oftmals per se als Opfer wahrnimmt, ohne dabei die Ambivalenzen und Widersprüchlichkeiten der Unterbringung aus Kindersicht ausreichend zu berücksichtigen (vgl. White 2012). Bis auf einzelne Ausnahmen (für den deutschsprachigen Raum vgl. World Vision 2016, Lewek \& Naber 2016; UNICEF 2017) ist wenig darüber bekannt, wie minderjährige begleitete Geflüchtete ihre Lebensverhältnisse wahrnehmen. Die genannten Studien beziehen sich eher auf die Perspektiven älterer Kinder und Jugendlicher. Die Ansichten und Praktiken von jungen, noch nicht schulpflichtigen Kindern in Massenunterkünften ist bislang ein wenig erforschtes Feld.

\section{Der Aufbau des vorliegenden Buches}

Als qualitativ-ethnografisch konzipierte Studie rückt die vorliegende Publikation das Handeln sehr junger Kinder in den Mittelpunkt des Interesses. Mittels einer explorativen Herangehensweise beschreibt sie, wie und auf welche Weise Kinder ihren Alltag in den Unterkünften leben. Für die Untersuchung der Alltagsorganisation wurden die Interaktionen der Kinder mit anderen Akteur*innen im Feld beobachtet, die institutionellen Praktiken analysiert und die rechtlichen Rahmenbedingungen im europäischen und nationalen Recht sowie übergeordnete internationale Übereinkommen wie die UN-Kinderrechtskonvention hinzugezogen.

\subsection{Fragestellung der Studie}

Die Studie basiert auf der Bearbeitung folgender Fragestellungen, die aus der teilnehmenden Beobachtung im Feld als zentral für das Verständnis vom Alltag junger Kinder in den Unterkünften für Geflüchtete heraus entwickelt wurden.

4 Für einen Überblick der englischsprachigen Literatur vgl. Kaukko et al. 2017. Für die Diskussion von Ambivalenzen und Widersprüchlichkeiten in der Unterbringung geflüchteter Kinder in Europa vgl. für einen Überblick in englischsprachigem Raum wie in Irland White 2012; für Skandinavien z. B. Eastmond \& Ascher 2011; Vitus \& Lidén 2010; Archambault 2012. 


\subsubsection{Raumerfahrungen}

Wie verhalten sich junge Kinder in diesen spezifischen Unterbringungsverhältnissen? Welche Strategien der Aneignung der Orte entwickeln sie? Als Ort wird eine spezifische Ordnung verstanden, die einer momentanen Konstellation von festen Punkten Stabilität verleiht (vgl. de Certeau 2006). Durch Bewegungen, Handlungen und den Faktor Zeit wird aus einem Ort ein Raum. Dieser ist sozial produziert und in seiner Bedeutung mehrdeutig und veränderlich. Raum ist nach de Certeau das Produkt sozialer Aushandlungsprozesse konfligierender und übereinstimmender Interessen und Aktivitäten (ebd.: 345).

Indem die Studie auf die Raumerfahrungen von Kindern fokussiert, rückt deren Umgang mit den spezifischen Orten in den Blick und es kann analysiert werden, was und wie Kinder in der erzwungenen Unterbringungsform ihren Alltag gestalten (können), ob und wie sie sich Raum aneignen, ihn umdefinieren, erweitern und verteidigen. Es geht um die Dimensionen, die Raum für Kinder in diesem spezifischen Kontext der Unterkünfte für Geflüchtete einnehmen kann.

\subsubsection{Handlungsvermögen und Handlungsfähigkeit von Kindern}

Die zweite Fragestellung der Studie fokussiert darauf, wie die soziale Akteur*innenschaft von Kindern in den Unterkünften für Geflüchtete beschrieben werden kann. Wie gestalten Kinder ihren Alltag als Kinder in den Unterkünften, eingebettet in die generationale Ordnung vor dem Hintergrund unsicherer bzw. prekärer Aufenthaltsverhältnisse? Welche Einschränkungen erleben sie, welche Möglichkeiten entwickeln sich, auf welche Ressourcen können Kinder zurückgreifen bzw. für sich mobilisieren und welche Reaktionen bringen ihre Bedürfnisäußerungen in diesem Kontext jeweils hervor? In der Analyse werden die spezifischen Merkmalsausprägungen der Kinder wie ihr Alter, ihr Status in der Geschwisterfolge und ihre Gesundheit berücksichtigt und als spezifische hemmende oder förderliche Einflussfaktoren in der Gestaltung ihres Alltagslebens als ,relationale Agency“ diskutiert (vgl. Bamler et al. 2010; Hungerland \& Kelle 2014). Hinzu kommen die jeweiligen biografischen Erfahrungen, die soziale Eingebundenheit und die materiellen, rechtlichen und kulturellen Verhältnisse sowie die Staatsangehörigkeit des Kindes und seiner Familie. 


\subsubsection{Gewalt und die Reproduktion von Machtverhältnissen}

Die dritte Fragestellung der Studie betrifft die Machtverhältnisse, in die geflüchtete Kinder eingebettet sind. Folgende Fragen entwickelten sich im Zuge der Erhebung: Durch welche Praktiken und Institutionen werden geflüchtete Kinder zu einer besonderen vulnerablen (Kinder-)Gruppe (gemacht)? Wie trägt der Ort der Unterkunft zu spezifischen Machtverhältnissen bei, die sich in Raumrepräsentationen (vgl. Lefebvre 1991) niederschlagen oder materialisieren? Inwiefern ist die Forschungspraxis an der Reproduktion asymmetrischer Machtverhältnisse beteiligt? Und schließlich: Wie positioniert sich die ethnografische Forschung zu den politisch-diskursiv umkämpften Fragen von Schutz, Versorgung und Beteiligung geflüchteter Kinder?

Die Studie ist das Ergebnis eines sich über 20 Monate erstreckenden qualitativ-empirischen Forschungsprojekts in drei Unterkünften verschiedener gemeinnütziger Träger in Berlin. Durchgeführt wurde die Studie von einem interdisziplinär zusammengesetzten Team mit unterschiedlicher sozialer und kultureller Herkunft. Es waren ausschließlich Forscherinnen an der Studie beteiligt, ohne dass dies konzeptionell beabsichtigt gewesen war. Das Sample der Studie umfasst zwei Gemeinschaftsunterkünfte und eine Erstaufnahmeeinrichtung in verschiedenen Bezirken in Berlin. Die Unterkünfte unterschieden sich in der Dauer ihrer Inbetriebnahme sowie in ihrer Größe und Beschaffenheit der Räumlichkeiten. Das Forschungsvorhaben wurde durch die finanzielle Förderung der Bertelsmann Stiftung, der Stiftung Deutsche Jugendmarke, des Paritätischen Landesverbands Berlin und des INIB e. V. ermöglicht.

\subsection{Aufbau des Buches}

Im ersten Teil stellt Anne Wihstutz das Forschungsdesign der Studie als in der sozialkonstruktivistischen Kindheitstheorie und kritischen Migrationsforschung verankert vor. Das Verhältnis von Kinderrechten und Asylpolitik wird in einem weiteren Beitrag von Anne Wihstutz in seiner Widersprüchlichkeit untersucht und als Ausdruck widerstreitender Diskurse über die politische Identität asylbegehrender Kinder diskutiert. Anschließend formuliert Hoa Mai Trần aus ethisch-reflektierter Perspektive Überlegungen zu den empirischen Erfahrungen im Forschungsprojekt.

Der zweite Teil umfasst vier empirisch basierte Beiträge. Sarah Fichtner und Hoa Mai Trần fokussieren in ihrem Beitrag auf Strategien von Kindern in der Aneignung, Verteidigung und Herstellung von Beziehungs-, Spiel- und Handlungsräumen in zwei Gemeinschaftsunterkünften. Über die dichte Beschreibung von drei Fallbeispielen diskutieren die Autorinnen die Handlungs- 
fähigkeit von sehr jungen Kindern als kontextbezogen und situationsbedingt relational. Penelope Scott und Thi Huyen Trang Le untersuchen in ihrem Beitrag die Bedeutung von Spiel bzw. wie Kinder sich in einer Erstaufnahmeeinrichtung Möglichkeiten zu spielen schaffen. Mit Bezug auf Lefebvre (1991) analysieren die Autorinnen, auf welche Weise Kinder an der Produktion von Raum als sozialem Prozess beteiligt sind. In Bezug auf das Erleben von Gewalt stellt Evelyn Schulz-Algie in ihrem Beitrag die Frage, was Gewalt bedeutet und was demnach der Schutz vor Gewalt in dem spezifischen Kontext von Unterkünften für geflüchtete Menschen heißen kann. Penelope Scott beschreibt die emotionalen und ethischen Herausforderungen ihrer Forschung mit Kindern in einer Erstaufnahmeeinrichtung. Dabei stellt sie die Forschungserfahrung als ganzheitlich und nachhaltig herausfordernd dar für das emotionale, wissenschaftlich-analytisch und politische Engagement der Forscherin und weist auf die Bedeutsamkeit dieser Erfahrung für den Erkenntnisgewinn hin.

Im dritten Teil fasst Anne Wihstutz die zentralen Erkenntnisse in Auseinandersetzung mit migrationspolitischen und kindheitstheoretischen Einschätzungen aktueller politischer Entwicklungen zusammen. Im Sinne engagierter Forschung schließt das Buch mit einem Positionspapier, das Forderungen an die politische und pädagogische Zusammenarbeit mit geflüchteten Kindern stellt.

\section{Literatur}

Abgeordnetenhaus (2015): Situation von Kindern, die in Notunterkünften leben. Schriftliche Anfrage der Abgeordneten Marianne Burkert-Eulitz (GRÜNE) vom 15. Dezember 2015, http://marianne-burkert-eulitz.de/wp-content/uploads/2016/01/s17-17618-Notunterkünfte.pdf (Zugriff 1.6.2017).

Archambault, Josée (2012): „It can be good there too": home and continuity in refugee children's narratives of settlement. In: Children's Geographies 10 (1), S. 35-48.

Bade, Klaus \& Oltmer, Jochen (2005): Flucht und Asyl seit 1990. Dossier Grundlagendossier Migration. Bundeszentrale Politische Bildung, 15.3.2005, https:// www.bpb.de/gesellschaft/migration/dossier-migration/56443/flucht-und-asylseit-1990 (Zugriff 9.7.2018).

Bundesamt für Migration und Flüchtlinge (BAMF): Das Bundesamt in Zahlen 2017. Asyl, http://www.bamf.de/SharedDocs/Anlagen/DE/Publikationen/Broschueren/bundesamt-in-zahlen-2017-asyl.pdf?_blob=publicationFile (Zugriff 21.6.2018).

Bundesministerium des Inneren (BMI) (2016): 890.000 Asylsuchende im Jahr 2015. Pressemitteilung 30.9.2016, https://www.bmi.bund.de/SharedDocs/pressemitteilungen/DE/2016/09/asylsuchende-2015.html (Zugriff 15.1.2018). 
Berliner Senat (2017): Pressemitteilung 11.7.2017, https://www.berlin.de/rbmskzl/ aktuelles/pressemitteilungen/2017/pressemitteilung.610650.php (Zugriff 13.7. 2017).

Berliner Senat (2017): Pressemitteilung 3.7.2017, https://www.berlin.de/laf/service/pressemitteilungen/2017/pressemitteilung.608344.phphttps://www.berlin. de/laf/service/pressemitteilungen/2017/pressemitteilung.608344.php download 13.7.2017 (Zugriff 13.7.2017).

Betz, Tanja \& Eßer, Florian (2016): Kinder als Akteure- Forschungsbezogene Implikationen des erfolgreichen Agency-Konzepts. In: Diskurs Kindheits- und Jugendforschung 11 (3), S. 301-314.

Bundesfachverband unbegleiteter minderjähriger Flüchtlinge e. V. (BumF) (2016): Fact- Finding zur Situation von Kindern und Jugendlichen in Erstaufnahmeeinrichtungen und Notunterkünften. Zusammenfassender Bericht November 2015 - Januar 2016. Mirjam Lewek und Tobias Klaus für den BumF e. V., beauftragt vom Deutschen Komitee für UNICEF e. V., Berlin, Februar 2016.

De Certeau, Michel D. (2006): Praktiken im Raum. Raumtheorie. Grundlagentexte aus Philosophie und Kulturwissenschaften, Frankfurt a. M.: Suhrkamp, S. 343353.

Eastmond, Marita \& Ascher, Henry (2011): In the Best Interest of the Child? The Politics of Vulnerability and Negotiations for Asylum in Sweden. In: Journal of Ethnic and Migration Studies 37 (8), S. 1185-1200.

Engler, Marcus \& Krause, Ulrike (2018): Globale Trends: Wachsende Herausforderungen für den globalen Flüchtlingsschutz. Beitrag im Blog NetzwerkFlüchtlingsforschung. https://fluechtlingsforschung.net/globale-trends-wachsendeherausforderungen-fur-den-globalen-fluchtlingsschutz/ (Zugriff 20.6.2018).

Fleischmann, Larissa (2016): 3 comments. Kommentar zum Beitrag Ulrike Krause „Das Label Flüchtling“. In: ZIB- Zeitschrift für internationale Beziehungen, 19.5.2016, https://zib-online.org/2016/05/19/das-label-fluechtling-homogenisierung-und-viktimisierung-durch-eine-globale-labelkonstruktion/ (Zugriff 20.6.2018).

Handelsblatt (2018): Die Flüchtlingspolitik spaltet Merkel und Seehofer erneut, https://www.handelsblatt.com/politik/deutschland/masterplan-vorstellung-verschoben-die-fluechtlingspolitik-spaltet-merkel-und-seehofer-erneut/22671026. html?ticket=ST-1417142-gKQ0LGebLdLB3JfAxfh0-ap2 (Zugriff 23.8.2018).

Holmes, Seth M. \& Castañeda, Heide (2016): Representing the ,European refugee crisis' in Germany and beyond: Deservingness and difference, life and death. In: American Ethnologist 43 (1), S. 12-24.

James, Alan \& Prout, Alan (Hrsg.) (1990): Constructing and Reconstructing Childhood: contemporary Issues in the Sociological Study of Childhood. London: Falmer Press.

James, Alison \& James, Alan (2012): Key concepts in Childhood Studies. Los Angeles: SAGE.

Kaukko, Mervi, Dunwoodie, Karen \& Riggs, Elisha (2017): Rethinking the Ethical and Methodological Dimensions of Research with Refugee Children. In: ZEP- 
Zeitschrift für internationale Bildungsforschung und Entwicklungspädagogik 40 (1), S. 16-21.

Kit-Agentur für verständliche Sprache (2018): Satz der Woche: „Wir müssen endlich unsere Grenzen wirksam sichern. Dazu gehört natürlich die Zurückweisung. Der Asyltourismus muss beendet werden. Deutschland darf nicht endlos auf Europa warten, sondern muss selbstständig handeln. Markus Söder, Twitter, 14.06.2018“", https://www.kit-agentur.de/satzderwoche/ (Zugriff 23.8.2018).

Krause, Ulrike (2016): Das Label ,Flüchtling': Homogenisierung und Viktimisierung durch eine globale Labelkonstruktion. In: ZIB- Zeitschrift für internationale Beziehungen, 19.5.2016, https://zib-online.org/2016/05/19/das-labelfluechtling-homogenisierung-und-viktimisierung-durch-eine-globale-labelkonstruktion/ (Zugriff 20.6.2018).

Lefebvre, Henri (1991): The Production of Space. Oxford: Wiley-Blackwell.

Sachverständigenrat deutscher Stiftungen für Integration und Migration, SVR (2015): Junge Flüchtlinge. Aufgaben und Potenziale für das Aufnahmeland. Kurzinformation des SVR-Forschungsbereichs 2015-2, https://www.svr-migration.de/publikationen/junge-fluechtlinge-aufgaben-und-potenziale-fuer-dasaufnahmeland/ (Zugriff 21.6.2018).

Sachverständigenrat deutscher Stiftungen für Integration und Migration, SVR (2017): Fakten zur Asylpolitik. 1. Halbjahr 2017. 23. August 2017, aktualisierte Fassung, https://www.stiftung-mercator.de/media/downloads/3_Publikationen/ 2017/August/SVR_Fakten_zur_Asylpolitik_Stand_August_2017.pdf (Zugriff 19.6.2018).

Sachverständigenrat deutscher Stiftungen für Integration und Migration, SVR (2018): Fakten zur Asylpolitik 2017. 31. Mai 2018, aktualisierte Fassung, https://www.svr-migration.de/wp-content/uploads/2018/05/SVR_Fakten_zur_ Asylpolitik.pdf (Zugriff 19.6.2018).

Tworek, Heidi (2018): Als ob Flüchtlinge Touristen wären. Süddeutsche Zeitung, 20.6.2018.

UNHCR (2018): Global Trends Forced Displacement in 2017, http://www.unhcr.org/statistics/unhcrstats/5b27be547/unhcr-global-trends-2017.html (Zugriff 20.6.2018).

Vitus, Kathrine \& Lidén, Hilde (2010): The Status of the Asylum-seeking Child in Norway and Denmark: Comparing Discourses, Politics and Practices. In: Journal of Refugee Studies Vol. 23 (1), S. 62-81.

Weinzierl, Ruth (2010): Der Asylkompromiss 1993 auf dem Prüfstand, Berlin: Deutsches Institut für Menschenrechte.

White, Allan (2012): „Every Wednesday I Am Happy“. Childhoods in an Irish Asylum Centre. In: Population Space Place 18, S. 314-326. 



\title{
Das Forschungsprojekt und sein Design
}

\author{
Anne Wihstutz
}

„Es gehört zu den Selbstverständlichkeiten der heutigen sozialwissenschaftlichen Kindheitsforschung, dass Kinder und Kindheiten nicht als natürliche Gegebenheiten betrachtet werden können, sondern durch die gesellschaftlichen Verhältnisse, sozialen Beziehungen und kulturellen Kontexte, in die sie eingebunden sind, wesentlich mitgeprägt werden“ (Liebel 2017: 11).

\section{Einleitung}

Es gehört zu den Grundannahmen der neueren Kindheitswissenschaften, dass Kinder eine eigene Sprache haben, um ihren Erfahrungen und eigenen Sichtweisen Ausdruck zu geben (vgl. z.B. Gandini 2011; Clark \& Moss 2011). Die UN-Kinderrechtskonvention formuliert es als ein Recht von Kindern, die eigene Meinung frei zu äußern und gehört zu werden (Artikel 12 UNKRK). Umso auffallender ist, dass den Erfahrungen und Perspektiven junger Kinder, die mit ihren Eltern in Unterkünften für Geflüchtete untergebracht sind, wenig Gehör geschenkt wird. Die Forschungsliteratur zu den Lebensverhältnissen in Unterkünften fokussiert eher auf die Perspektiven älterer Kinder und Jugendlicher (für den deutschsprachigen Raum vgl. World Vision 2016, Lewek \& Naber 2016; UNICEF 2017). Hier setzt die Studie an, um einen Beitrag zur Schließung dieser Forschungslücke zu leisten. In dem vorliegenden Beitrag stelle ich das Forschungsprojekt vor. Im ersten Kapitel werden die forschungsrelevanten theoretischen Bezüge aus der sozialkonstruktivistischen Kindheitsforschung und der kritischen Flucht- und Migrationsforschung skizziert. Ich definiere Kinder als soziale Akteure, die ihre Kindheit in einer Vielfalt von Lebenslagen und Lebenswelten (mit)gestalten. Mit dem Begriff der doppelten Viktimisierung umreiße ich knapp den Diskurs über junge geflüchtete Kinder. Der Beitrag skizziert das der Forschung zugrundeliegende Verständnis von Alltag im Kontext der spezifischen Verhältnisse der Unterkünfte für Geflüchtete. Im zweiten Kapitel stelle ich die Methodologie der Ethnografie vor, in dem ich sie als spezifische Forschungshaltung und -methode einführe. Ich stelle die Besonderheit der Gleichzeitigkeit von Handlungsinvolviertheit und Datenerhebung heraus. Dieses diskutiere ich als Herausforderung für die For- 
scherinnen im Feld und als Chance für die Verifizierung der Güte der Dateninterpretation. Die Forschung wird als ein Prozess vorgestellt. Im Verlauf von Datenerhebung und -analyse konkretisiert sich die Fragestellung. Die induktiv entwickelte Kategorie Raum als Produkt sozialer Prozesse führe ich in ihren verschiedenen Dimensionen ein. Ich beschreibe kurz das Sample der Studie, das drei Unterkünfte umfasst. Abschließend benenne ich spezifische feldbezogenen Herausforderungen und Grenzen der Studie.

\section{Theoretische Rahmungen}

\subsection{Sozialkonstruktivistische Kindheitstheorie}

Die vorliegende Studie ist verankert in der sozialkonstruktivistischen Kindheitstheorie. Spätestens seit der grundlegenden Publikation von James, Jenks und Prout „Theorizing Childhood“ (1998) ist es Konsens in der Kindheitsforschung, Kinder als Subjekte wahrzunehmen, die eingebettet in spezifische Verhältnisse eigene Ideen und Interpretationen ihrer (Lebens-)Situationen zum Ausdruck bringen (vgl. James et al. 1998; James 2009). Als soziale Akteur*innen setzen Kinder sich von Beginn an mit ihrer Umwelt auseinander. Bereits als Säuglinge sind sie darauf angewiesen, auf ihre (soziale) Umwelt einzuwirken und von dieser in ihren Grundbedürfnissen wahrgenommen zu werden (vgl. Wells 2009). Wie Kinderleben und Kindheit gesellschaftlich verhandelt und gestaltet werden, ist im Kontext historischer, soziokultureller sowie ökonomischer Prozesse und Entwicklungen zu betrachten. Mit der Herausbildung der Moderne hat sich in Europa ein Bild von Kindheit als Schutz- und Vorbereitungsphase, die über spezifische Institutionen abgesichert wird, durchgesetzt (vgl. Wihstutz 2018). Zu ihrer physiologisch bedingten Angewiesenheit auf Schutz und Unterstützung ist es vor allem die strukturelle Abhängigkeit, die die soziale Gruppe Kinder rechtlich, ökonomisch, politisch und sozial als gesellschaftlich marginalisiert positioniert. In dieser Position sind sie mit spezifischen Rechten und Pflichten ausgestattet, die ihre Handlungsspielräume erweitern oder auch einschränken.

Die generationale Ordnung von Gesellschaft bringt die soziale Gruppe der Kinder hervor und sichert das soziale Phänomen „Kindheit“" strukturell ab (vgl. Qvortrup 1994). Als soziale Strukturkategorie sind spezifische Aufgaben- und Problembereiche mit Kindheit verbunden. Dabei gestalten sich die Lebenswelten von Kindern in Verbindung mit ihrem Alter, ihrem Geschlecht, ihrer Gesundheit, ihrer soziokulturellen Herkunft und ihrem Aufenthaltsort heterogen und plural. Als soziokulturelles und historisches „Konstrukt“ ist Kindheit un- 
terschiedlich gestaltet. Kinder als soziale Akteur*innen sind dabei eingebunden in Machtbeziehungen und über Interaktionen mit ihrer Umwelt tragen sie zur Konstitution und Reproduktion sozialer Ungleichheiten bei. So sind Kinder an Prozessen generationalen Ordnens beteiligt, an ihrer Reproduktion und Veränderung, ohne die generationale Ordnung jedoch aufheben zu können. Diese gilt vielmehr als Gesellschaften ordnende Struktur (vgl. Qvortrup 1994; Mierendorff 2010). Bühler-Niederberger hat dafür den Begriff der „Akteurschaft als Kind“" geprägt (Hungerland \& Kelle 2014; Bühler-Niederberger 2011).

„Children from early ages constitute, perpetuate and negotiate normalizing discourses around their identities, and are actively regulating not only their own behaviour accordingly, but also that of others around them" (Robinson \& Jones Diaz, zitiert in Machold 2015: 28).

Identitäten werden in und durch Machtverhältnisse in Diskursen produziert. Sie entwickeln sich in einem Spannungsfeld kontinuierlicher Aushandlungen und sind weder fixiert noch als gleichbleibend vorzustellen, vielmehr tragen Hierarchisierungs- und Diskriminierungsprozesse, Ausschluss- und Inklusionserfahrungen und -strukturen dazu bei, dass Identitäten sich fortwährend weiterentwickeln und somit als ein offener Prozess zu verstehen sind. Statt von einer Identität ist von der Pluralität von Identitäten und der gleichzeitigen $\mathrm{Zu}$ gehörigkeit zu mehreren Gruppen auszugehen, die sich verändern können (vgl. Mecheril 2004; Liebel 2018). Auch als Gruppe können Kinder Interessen haben, die nicht nur gegenüber Erwachsenen unterschiedlich sind, sondern aus ihrem „Flüchtlingsstatus“ heraus gruppenspezifische Bedarfe gegenüber der aufnehmenden Gesellschaft formulieren, die sie mit geflüchteten Erwachsenen teilen und sie von den Interessen einheimischer Kinder unterscheiden.

Im Kontext von Kindheit und Flucht sind die Sicherung von Schutz und Versorgung von Kindern als gesellschaftliche Aufgaben naheliegend. ${ }^{1}$ Doch ein darauf reduziertes Verständnis wird dem veränderten Verständnis von Kindern als eigenständige Rechtssubjekte mit Anspruch auf gesellschaftliche Beteiligung nicht gerecht. Das Verhältnis von Schutz und Partizipation ist spannungsgeladen. Die Kontroverse kreist um Fragen der Voraussetzungen und Bedingungen gesellschaftlicher Partizipation von Kindern und führt schließlich zur Frage nach dem Kindheitsbild bzw. Menschenbild, das implizit im verhandelten Partizipationsverständnis steckt (vgl. Cockburn 2007). Während die UN-Kinderrechtskonvention (UNKRK) als ein Meilenstein in der Geschichte der Anerkennung von Menschenrechten für Kinder gilt, ist mit ihr eine rechtliche Sonderstellung von Kindern verknüpft, die nicht widerspruchs-

1 Die im Jahr 1989 von der Vollversammlung der Vereinten Nationen verabschiedete Konvention zu den Rechten des Kindes (UN-Kinderrechtskonvention, UNKRK) nennt drei grundlegende Prinzipien im Zusammenhang mit den Rechten von Kindern: Beteiligung, Versorgung und Schutz. 
frei diskutiert wird (vgl. Bhabha 2006). ${ }^{2}$ Kritiker*innen monieren, dass die UNKRK im dichotomen Denken der europäischen Moderne verhaftet bleibe. Statt einen ganzheitlichen Ansatz zu verfolgen, reproduziere die UNKRK polare Gegensätze wie im Verhältnis von Kindern und Erwachsenen, Privatheit und Öffentlichkeit, Natur und Kultur, Irrationalität und Rationalität, Abhängigkeit und Unabhängigkeit, Passivität und Aktivität, Inkompetenz und Kompetenz sowie Spiel und Arbeit (vgl. Prout 2005: 10; Liebel 2017). Entsprechend werden Kinder in der UN KRK vorrangig als isolierte Individuen konzeptualisiert, unter Vernachlässigung ihrer sozialen Eingebundenheit und Einbettung in interdependente Beziehungen (vgl. Wihstutz 2017; Pupavac 2001; Liebel 2017). Derart werden die Grautöne und Zwischenräume, Übergänge sowie die heterogenen und vielschichtigen miteinander verwobenen gesellschaftlichen Einflussfaktoren in Bezug auf Kinder und Kindheit nicht ausreichend berücksichtigt. Die Betrachtung des Kontextes, in den Kindheit eingelassen ist, ist für dessen Verständnis und Ausformung grundlegend. So kann es auch nicht die eine „Flüchtlingskindheit“ geben. Vielmehr sind jeweils die konkreten Verhältnisse zu untersuchen, die das Leben von geflüchteten Kindern strukturieren und ihr Handlungsvermögen beeinflussen.

\subsubsection{Agency}

Es ist Konsens in der neueren Kindheitstheorie, dass die (Hervorbringung der) Handlungsfähigkeit von Akteur*innen in Bezug auf konkrete Situationen zu analysieren ist (vgl. James 2009). Handlungsvermögen ist biografisch, strukturell und situational bedingt sowie von verschiedenen Einflussfaktoren wie subjektiven Interessen abhängig. Scherr spricht in dem Maße von Agency, „,wie die biografisch vorgängigen Prozesse, die zur Formierung individueller bzw. kollektiver Dispositionen geführt haben sowie die aktuell vorgefundenen situativen Bedingungen nicht alternativlos festlegen, was als nächstes geschieht.“ (Scherr 2012: 109). Es ist von einer Handlungsfähigkeit der Kinder $\mathrm{zu}$ sprechen, wenn Kinder (als soziale Akteur*innen) eigene Interessen und Bedürfnisse in ihren Entscheidungen, im Handeln zum Ausdruck bringen, die

2 Die UN-Kinderrechtskonvention knüpft Partizipation wie das Recht des Kindes, sich eine Meinung zu bilden und diese frei zu äußern, an seine altersgebundene Fähigkeit und „Reife“ (Artikel 12 Berücksichtigung des Kindeswillens). Einschränkung erfährt die Berücksichtigung des Kindeswillens dadurch, dass dieser, ,angemessen“ zu berücksichtigen ist. Nicht Kinder selbst urteilen über ihre Reife, sondern Erwachsene definieren die Reife und die Angemessenheit kindlicher Fähigkeiten, schließlich haben Kinder die Kinderrechte nicht den Erwachsenen abgerungen oder sind von Kindern erstritten worden (vgl. Cordero Arce 2015). Demzufolge ist die UN-Kinderrechtskonvention auch nur bedingt hilfreich in dem Bestreben, die gesellschaftliche Beteiligung der spezifischen sozialen Gruppe Kinder zu ermöglichen bzw. zu sichern (vgl. auch Liebel 2018). 
als Alternativen zum Bestehenden gedacht werden, und der Verlauf der Situation nicht bereits vorab durch Bedingungen festgelegt ist. Agency ist demnach immer auch zeitlich bzw. temporal eingebettet zu denken. Bei Emirbayer und Mische heißt es diesbezüglich, ,[t]he agentic dimension of social action can only be captured in its full complexity, [] if it is analytically situated within the flow of time" (Emirbayer \& Mische 1998: 963). In der sozialwissenschaftlichen Kindheitsforschung hat sich das theoretische Konzept Agency als Handlungsfähigkeit bzw. Handlungsvermögen durchgesetzt, um den aktiven Beitrag von Kindern an der Gestaltung ihrer sozialen Welten und in der Gesellschaft anzuerkennen (vgl. Betz \& Eßer 2016; Hungerland \& Kelle 2014: 227). Agency von Kindern wird in sozialen Handlungen, Praktiken und Diskursen hervorgebracht.

„Children are and must be seen as active in the construction and determination of their own social lives, the lives of those around them and of the societies in which they live. Children are not just the passive subjects of social structures and processes" (Prout \& James 1990: 8).

\subsection{Flüchtlings- und Viktimisierungsdiskurse}

Der Forschungsgegenstand der vorliegenden Studie umfasst das Leben junger Kinder und ihrer Familien in Unterkünften für Geflüchtete. Eine solche Fokussierung erfordert sowohl konzeptionell als auch hinsichtlich der praktischen Folgen eine Auseinandersetzung mit der zugrunde liegenden Kategorie Geflüchtete und den damit verknüpften Diskursen. In Verbindung mit der jeweiligen aktuellen Ausländer- und Asylpolitik sind die Kriterien für die Zuerkennung des Flüchtlingsstatus definiert. Das Asylsystem schafft Hierarchien unter Schutzsuchenden und unterscheidet zwischen „legitimen Flüchtlingen“ und solchen, die als „Scheinasylanten“" aus wirtschaftlichen Gründen als nicht „echt“ gelten. Wer als „Flüchtling“ wie verhandelt wird, ist entsprechend politisch-rechtlich und sozial konstruiert (vgl. Niedrig \& Seukwa 2010). Als politisch-rechtliches und soziales Konstrukt erfüllt die Kategorie Flüchtling spezifische Funktionen im gesellschaftlichen Miteinander. Diese gilt es im Folgenden knapp zu skizzieren und ihre Bedeutung für das Leben geflüchteter Kinder zu untersuchen.

Aus postkolonialer Perspektive analysieren Niedrig und Seukwa die Bedeutung des Flüchtlingsbegriffs für die Aufnahmegesellschaft sowie für die Betroffenen selbst. „Ein ,echter Flüchtling“ wird als ,hilfloses, unschuldiges Opfer" imaginiert" (Niedrig \& Seukwa 2010: 185). Gendertheoretisch gesprochen sei der „echte Flüchtling“ mit „weiblichen“ Attributen versehen: ,passiv, hilflos, anspruchslos/bescheiden, leidensfähig, zu Anpassung und Unterord- 
nung bereit" (ebd.). Diskursanalytisch beschreiben Niedrig und Seukwa das Verhältnis von Aufnahmegesellschaft und Staat auf der einen Seite und „Flüchtlingen“ auf der anderen Seite als ein Beziehungsdreieck zwischen „Täter - Opfer - Retter“ (ohne dass jede Position immer auch besetzt sein müsse ${ }^{3}$, vgl. Niedrig \& Seukwa 2010). In einer solchen Dreiecksbeziehung funktioniere der Flüchtlingsbegriff "für die national kodierte Selbstdeutung" derart, dass das Aufnahmeland Deutschland sich entweder in der Position des „Retters“ (der „wirklich Verfolgten“) oder des „Opfers“ (von „Asylmissbrauch“) positioniere (Niedrig \& Seukwa 2010: 185).

Im Sinne dieser Dreiecksfigur verkörpert das geflüchtete Kind das unschuldige Opfer schlechthin, und somit bietet sich anderen die Gelegenheit, sich als „Retter" positionieren zu können. Die strukturell und entwicklungsbedingte Abhängigkeit des Kindes als „Kind“ sowie seine gesellschaftliche Positionierung als „Opfer-Flüchtling“ tragen mit zum Diskurs einer doppelten Viktimisierung geflüchteter Kinder bei (vgl. Pupavac 2008). Derart werden sein Handlungsvermögen sowie sein Subjektstatus nicht ausreichend berücksichtigt. Ebenso kann eine Viktimisierung von Geflüchteten im Allgemeinen und von Kindern im Besonderen für die eigene moralische Aufwertung funktionalisiert werden. ${ }^{4}$ Bühler-Niederberger analysiert, wie die Figur des unschuldigen Kindes für diverse religiöse, gesellschaftliche und politische Zwecke instrumentalisiert wird: „Die Macht der Unschuld wird aus den Kindern gewonnen, aber sie ermächtigt andere. Sie hat mit real lebenden Kindern nichts zu tun" (Bühler-Niederberger 2005: 126). An dieser Stelle sei darauf hingewiesen, dass es nicht darum geht, die realen Leidenserfahrungen und Strapazen der Flucht von Kindern zu verharmlosen oder abzustreiten, dass Kinder und ihre Eltern auch Opfer von Krieg und Vertreibung, erzwungener Flucht und Leidende sind. Vielmehr ist es wichtig, die Prozesse von Zuschreibungen und Selbstpositionierungen von Akteur*innen zu erkennen und der Analyse zugänglich zu machen.

In meiner Erläuterung der Dreiecksstruktur von Niedrig und Seukwa fehlt bislang die Figur des Täters. Den beiden Autor*innen zufolge werden unter der Kategorie des Täters ausschließlich jene Geflüchteten diskursiv gefasst, die als ,aktiv, durchsetzungsfähig, fordernd, tatkräftig, auf den eigenen Vorteil bedacht, etc." von der Mehrheitsgesellschaft wahrgenommen werden (Niedrig/Seukwa 2010: 185). Eine Person, die sich nicht wie oben beschrieben als Opfer verhhalte, sei demzufolge kein „echter Flüchtling“, sondern „Schein-

3 Das Beziehungsdreieck wird von Niedrig und Seukwa nicht gegendert.

4 Nach Bühler-Niederberger (2005) hat das unschuldige „Opferkind“ eine Funktion für moralische Höherbewertung der darauf bezogenen ,guten“ Erwachsenen - und damit besteht in der Praxis wenig Interesse daran, die Figuration aufzulösen. Bühler-Niederberger analysiert, wie das Kind ,naturalisiert“ und schlechthin zur moralischen Instanz erhoben und für spezifische andere Interessen in Religion, Zivilgesellschaft und Politik in Anspruch genommen wird (vgl. Bühler-Niederberger 2005). 
asylant" und damit eher „Täter“, der die Hilfsleistungen der aufnehmenden Gesellschaft missbrauche (vgl. Niedrig/Seukwa 2010).

Wenn von einem solchen (diskursiv begründeten) Täter-Opfer-RetterDreiecksverhältnis auszugehen ist, sieht sich die akteur*innenzentrierte Forschung mit Geflüchteten, die geflüchtete Kinder weder romantisierend als Held*innenen (oder Täter*innen) darstellen noch dazu beitragen will, sie auf den Status hilfloser Opfer zu reduzieren (vgl. Kaukko et al. 2017), mit Fragen konfrontiert. Methodologisch steht die Forschung vor der Herausforderung, sensibel die Bedürfnisse und Interessen von Kindern und Eltern in diesen besonderen, verunsichernden Verhältnissen wahrzunehmen, auf diese einzugehen und ihre strukturell bedingte Vulnerabilität anzuerkennen. Vor dem Hintergrund dieser Überlegungen wurden im Forschungsprojekt Methoden der Prozessbeobachtung und -beteiligung gewählt, die versprachen alltägliche Situationen und Handlungsvollzüge der Akteur*innen im Feld für die Forscherinnen am eigenen Leib erfahrbar und in ihrer spezifischen (Handlungs-)Logik nachvollziehbar zu machen. Forschungspragmatisch galt es auf den konkreten Kontext bezogen empirisch zu untersuchen, ob und wie Kinder sich Möglichkeiten erschließen, auf ihre soziale Verhältnisse Einfluss zu nehmen, und welche Faktoren sich dabei als förderlich oder hinderlich zeigen.

\subsubsection{Zum Alltagsbegriff im Zustand der Ausnahme}

Dreh- und Angelpunkt der Studie ist die Alltagsorganisation bzw. der Alltag junger Kinder und ihrer Familien in Erstaufnahmezentren und Gemeinschaftsunterkünften. Nicht nur aus forschungspragmatischen Gründen wurde dieser Fokus gewählt: Aufgrund asyl- und aufenthaltsrechtlicher Bestimmungen sind die Bewegungs- und Aufenthaltsräume Geflüchteter stark eingeschränkt. Für die unter Sechsjährigen ist der Besuch einer Bildungsstätte nicht verpflichtend vorgesehen. Zudem legen Studien nahe, dass der Besuch von Kindertageseinrichtungen für geflüchtete Kinder bundesweit nicht einheitlich geregelt und ein Zugang zu frühkindlichen Bildungseinrichtungen schwierig bzw. kaum gegeben ist (vgl. DJI 2017; GEW 2013). Mit der Fokussierung auf den Ort „Unterkunft" unternimmt die vorliegende Studie den Versuch, den Alltag von jungen geflüchteten Kindern mit ungeklärtem Aufenthaltsstatus zu erheben.

Voß beschreibt Alltag als einen Handlungsmodus, also als ,gewöhnliches“ oder ,normales“ Tun, das von fest- oder feiertäglichem Handeln abgesetzt werden kann (vgl. Voß 2000: 33). Es interessieren die Praxen des Alltags, das Selbstverständliche im Miteinander, wie sich die Akteur*innen durch ihr Handeln im sozialen Gefüge der Unterkunft positionieren. Über das Handeln werden subjektive Situationsdeutungen der Akteur*innen, ihr Welterleben - Voß nennt es Existentialerfahrung (vgl. Voß 2000) - für die Forscherinnen im Feld erfahr- und interpretierbar. Dabei sind das Handeln und die Art und Weise, wie 
Welt erlebt wird, für die Akteur*innen so selbstverständlich und unmittelbar, dass ihr Agieren und Erleben ihnen selbst meist nicht ohne weiteres zugänglich sind. Gerade deshalb ist dieser Modus des Alltäglichen und seine Verankerung im Hier und Jetzt für die wissenschaftliche Analyse so aufschlussreich (ebd.).

Der Fokus auf Alltagshandeln unterstellt die Möglichkeit eines alltäglichen Lebens, in dem sich Routinen im Handeln entwickeln können. In Anbetracht der unklaren und unsicheren Aufenthaltsverhältnisse geflüchteter Kinder und ihrer Eltern muss die Untersuchung zeigen, inwiefern sich ein derartiges Alltagshandeln beobachten lässt. Welche Möglichkeiten der Alltagsgestaltung entwickeln junge Kinder in Verhältnissen, die als Lager (vgl. Agamben 2002), totale Institution (vgl. Goffman 1973) in der Literatur diskutiert werden bzw. allgegenwärtiger Beobachtung (vgl. Foucault 2005) ausgesetzt sind (vgl. Schäfer 2015)? Welche Bedeutung haben Kinderrechte in einer Welt, in dem die Ausnahme zur Norm geworden und von dieser ununterscheidbar geworden ist (vgl. Agamben 2002: 179)? Mit der Fokussierung auf den Alltag von jungen Kindern in Unterkünften für Geflüchtete werden normative Annahmen von Kindheit, wie sie diskursiv in Deutschland verhandelt werden und sich im Recht materialisiert haben, in Bezug auf diese spezifischen Kindheiten untersucht. Die sozioökologische, lebensweltanalytische Perspektive (vgl. Bamler et al. 2010) der Studie verfolgt einen ressourcen- und kompetenzorientierten Ansatz. Die Autorinnen interessieren sich für institutionelle und alltägliche Praxen und Routinen der Akteur*innen und ihre Selbstpositionierungen in diesen konkreten Verhältnissen. Von Forschungsinteresse sind darüber hinaus die Zugänge, die sich Akteur*innen zu gesellschaftlichen Ressourcen und Rechten wie Teilhabe (im Sinne von Zugang zu frühkindlicher Bildung), Versorgung (z. B. Gesundheitsversorgung) und Schutz (vor Gewalt und Diskriminierung) erschließen.

\section{Ethnografische Forschung}

Ethnografie kann nach Hitzler verstanden werden ,als Forschungsprogramm, das darauf abzielt, andere Lebensweisen, Lebensformen, Lebensstile so zu sagen ,von innen' her zu verstehen, d. h. ,fremde Welten' auf ihren Eigen-Sinn hin zu erkunden“. Empirisch ist die Ethnografie, „eben an den Erfahrungen, die Menschen machen“, stark deskriptiv orientiert (Hitzler 2011: 48). Friebertshäuser und Panagiotopoulou (2009) definieren Ethnografie bzw. ethnografische Feldforschung als eine „Forschungstradition, die Menschen in ihrem Alltag untersucht, um Einblicke in ihre Lebenswelten und Lebensweisen zu gewinnen sowie ihre Sinndeutungen und Praktiken kulturanalytisch zu erschließen“" (ebd.: 301). 
Konstitutiv für die Ethnografie sind das Interesse an den „diskursiven und stummen Wissens- und Praxisformen“ (Breidenstein et al. 2013: 7) und eine sozialwissenschaftliche Haltung der Neugier, die von der Offenheit für das Unerwartete geleitet ist. Selbstverständliches und Alltägliches wird in der ethnografischen Herangehensweise „,befremdend“ analysiert (Amann \& Hirschauer 1997: 12). Die Annahme, Gesellschaft „unmittelbar verstehen“ zu können als „vertrauter, verstandener und auch verfügbarer[] Forschungsgegenstand“, gilt als Illusion der Sozialwissenschaft (Bourdieu \& Wacquant 1996: 280). Der Ethnografie geht es darum, soziale Lebenswelten, soziale Praktiken und institutionelle Verfahren empirisch zu erheben, indem die Forschenden sich im Feld regelmäßig und über längere Zeiträume aufhalten und teilnehmend beobachten. Damit entsteht eine Parallelität von Handlungsinvolviertheit und Datenerhebung. Was im Feld, in der Situation eine besondere Herausforderung darstellt, ist gleichermaßen Bedingung, um die Güte oder Qualität der Dateninterpretation rekonstruieren zu können. Anders als in retrospektiv, also Expost-Situationen sind Forschende darauf angewiesen, in situ und in actio Daten zu erheben. Die Forschenden selbst werden zum Forschungsgegenstand. Sie sind personale Aufzeichnungsinstrumente (vgl. Amann \& Hirschauer 1997a: 25). Das birgt Vorzüge gegenüber technischen Aufzeichnungsgeräten, aber auch Einschränkungen (vgl. Flick 1999). Menschen sind vergesslich, und ihre Eigenschaften sind nicht zu standardisieren, aber sie sind flexibel und kommunikativ. Während technische Forschungsinstrumente unbeeindruckt (weiter) dokumentieren, sind Forschende nicht unerschütterbar. Sie verfügen über Empathie. Als Menschen können sie Signale wahrnehmen, die weder über die Kamera noch mit einem Sprachaufnahmegerät in der gleichen Komplexität registriert werden können. Aus ihrem Situationserleben verfügen die Forschenden über Mehr-Wissen. Die teilnehmende Beobachtung ist an die Logik sozialer Prozesse gebunden. Die Forscher*innen selbst, ihr physisches Erleben der Situation (z. B. Riechen, Schmecken, Hören, Sehen, Spüren), ihre Gedanken und Gefühle in der Situation werden zum Gegenstand forschungsethischer Reflexionen. Damit setzen sich die ethnografisch arbeitenden Forscher*innen in einer besonderen Weise dem Untersuchungsfeld aus, das - manchmal fundamental - verunsichern kann (vgl. Amann \& Hirschauer 1997: 19). ${ }^{5}$ Die „Gleichörtlichkeit“" und „Gleichzeitigkeit“ (Breidenstein et al. 2013: 40) von Beobachtung und sozialem Geschehen sind Kernbestandteile ethnografischer Forschung.

„Die Ethnographie hat es stets mit perspektivisch gebrochenen Feldern zu tun, in denen parteiliche Versionen miteinander konkurrieren und sowohl multiple ,Perspektivübernahmen' als auch dezidiert eigenständige Versionen verlangen“ (Amann \& Hirschauer 1997: 23 f).

5 Der konkrete Umgang mit diesen Herausforderungen wird unter anderem in den Beiträgen von Trần und Scott ausführlich diskutiert. 
Es geht in der Ethnografie nicht darum, die Interpretationen, Meinungen und kognitiven Wissensbestände der Forschungsteilnehmer*innen über Interviews im Sinne von Selbstbeschreibungen zu erheben, sondern darum, die in der Praxis eingelagerten Wissensbestände zu erfahren. Es ist die „befremdete“ Beobachterin, die lokales Wissen expliziert (erklärt), weil es den Teilnehmer*innen von Handlungen als inkorporiertes Wissen und selbstverständliche Routine weder in der Situation noch auf Nachfragen sprachlich verfügbar ist. Die Weltsichten der Anderen werden von der Ethnografin als gelebte Praxis erkannt und erklärbar. Dabei handelt es sich um partielle Wahrheiten (,,partial truths" nennt es Clifford, zitiert nach Amann \& Hirschauer 1997: 23) oder um ein Spannungsverhältnis der Perspektiven, denn eine Praxis als Praxis zu erkennen kann nur, wer nicht in die durch sie gestellten Handlungsprobleme involviert ist. Ähnliches gilt für die Befremdung der eigenen Felderfahrungen der Forscherinnen in der vorliegenden Studie im Rahmen von Supervisionssitzungen und im reflektierenden Austausch im Team. Dabei gilt es zu differenzieren zwischen dem Verstehen als Teilnehmerin und als Beobachterin (siehe auch den Beitrag von Scott in diesem Band). Burke nennt es ,perspective by incongruity“ (Burke, zitiert nach Amann \& Hirschauer 1997: 24).

Modus Vivendi der ethnografischen Forschung ist die Reaktivität im Feld. In jedem spezifischen Feld herrscht eine eigene, diesem Feld immanente Ordnung oder auch Soziologik, die spezifische Verhaltens- und Beobachtungsweisen erforderlich machen (vgl. Amann \& Hirschauer 1997: 20).

„Sinnvolle Beobachtungseinheiten, Informationsauswahl und Datentypen können nur zum Teil geplant werden und müssen in Anpassung an die Eigenschaften des Feldes erst herausgefunden werden. Dafür braucht es zunächst den Zugang, den das Feld gewährt (oder nicht), sowie Zeit, sich im Feld zu orientieren“" (Breidenstein et al. 2013: 50).

In diesem Sinne ist die Datenerhebung zunächst aus einem „gleichgültigen“ und ,schielenden“ Blick heraus zu organisieren.

Im Rahmen der Studie zum Alltagsleben bestand die Herausforderung darin, die Soziologik durch die schrittweise Positionierung und „Eichung“ der Ethnografin in der jeweiligen Unterkunft handhabbar und erkenntnisgenerierend nutzbar zu machen. In diesem Sinne ist die Ethnografie auch eher als eine Forschungsstrategie und -haltung zu beschreiben denn als Technik oder geregelte Methode, in immer gleich anzuwendender Verfahrensweise (vgl. Breidenstein et al. 2013: 8).

Die im Feld erhobenen Daten und Notierungen macht die Ethnografin zum Material der soziologischen Analyse und bezieht dabei die sinnlich und selbsterlebte Lebenswirklichkeit mit ein. In der Forschung mit Kindern steht die Protokollierung vor der Herausforderung, dass das Aufgeschriebene und Skizzierte das besondere Interesse der Kinder erwecken kann. Gallacher und Gallagher beschreiben, wie ihre eigene ethnografische Forschung durch Kinder 
partizipativ gestaltet wurde: Kinder eigneten sich Forschungsinstrumente (Notizbücher) an und lenkten die Forschung durch Interventionen in die Richtung, die sie interessierte (vgl. Gallacher \& Gallagher 2008). Gallacher und Gallagher diskutieren die Interventionen der Kinder weniger als Störungen oder Problem, sondern als nicht geplante und höchst effektive Form der Partizipation von Kindern an Forschung.

\subsection{Die empirische Erhebung}

Die Erkenntnisse der vorliegenden Studie beziehen sich auf drei Unterkünfte unterschiedlichen Typs in Berlin. Eltern und Kinder wurden in ihrem Alltag begleitet, während sie auf den Entscheid über ihr Asylgesuch durch die zuständigen Behörden warteten. Die Untersuchung bezieht sich ausschließlich auf Aspekte des Zusammenlebens in den Unterkünften, sie ist insofern räumlich und lokal begrenzt. Die finanzielle Ausstattung des Projekts erforderte die Einschränkung des Feldaufenthalts auf acht Monate sowie die Begrenzung auf die Lokalität der Unterkünfte. Erst die lokale Begrenzung ermöglichte den Ethnografinnen die teilnehmende Beobachtung des Alltags von Kindern und ihren Familien. Im Fokus der Untersuchung stehen Kinder, die noch nicht der Schulpflicht unterliegen und damit ihren Lebensmittelpunkt physisch in den Unterkünften haben.

Die Datenerhebung und Dateninterpretation war derart konzipiert, dass sie im Tandem zwischen einer erfahrenen Ethnografin und einer studentischen Mitarbeiterin durchgeführt werden konnten. Die Tandems waren hinsichtlich der vertretenen beruflichen Disziplinen, ihrer soziokulturellen Herkunft und ihrer Sprachkompetenzen komplementär zusammengesetzt. Eine solche $\mathrm{Zu}-$ sammensetzung der einzelnen Tandems sollte der (Selbst-)Reflexion und Sensibilität für Diskriminierungsstrukturen im Feld dienlich sein und den Blick für die eigenen (forschungsrelevanten) Verstrickungen in Machtverhältnisse öffnen (zur Auseinandersetzung mit der Umsetzung auch forschungsethischer Fragen siehe den Beitrag von Trần ).

\subsection{Das Sample}

Es wurden in Unterkünften unterschiedlicher Betreiber Daten generiert. Das Sample umfasst eine Erstaufnahmeeinrichtung und zwei Gemeinschaftsunterkünfte in innerstädtischer Lage und im Randbezirk von Berlin. Je nach Unterbringungskapazität der Einrichtung lebten zwischen 100 und 515 Personen 
dort. Während des Erhebungszeitraums waren dort insgesamt zwischen 1248 und 1387 Menschen untergebracht. Sowohl allein geflüchtete Erwachsene als auch Familien mit Kindern und Alleinerziehende lebten in den Unterkünften. Das Forschungsprojekt begleitete von Mai 2016 bis Februar 2017 zwölf Kinder im Alter von drei bis sieben Jahren aus sieben Familien. Sie kamen aus dem Iran, aus Afghanistan und Bosnien. Der Aufenthaltsstatus dieser Familien war zum Zeitpunkt der Erhebung unsicher.

\subsection{Zugang und forschende Zusammenarbeit mit Kindern und Eltern}

In der Kontaktaufnahme mit Eltern und Kindern wurde ein offener Zugang für alle interessierten Familien gewählt, ohne Vermittlung durch Mitarbeiter*innen der Einrichtungen. Derart sollte der Eindruck vermieden werden, dass die Forschung eine Auftragsforschung der Unterkunftsleitung oder des Trägers sei. Die Einflussnahme durch (sozialpädagogische) Mitarbeiter*innen auf das Sample sollte somit vermieden werden. Mit Unterstützung externer Sprachmittler*innen wurde Eltern und Kindern das Forschungsprojekt vorgestellt. Potentiell Interessierte erhielten Handzettel in den Sprachen Farsi und Arabisch mit Informationen über das Anliegen, das Ziel und die Vorgehensweise der Forschung, außerdem stellten sich die Personen, die die Forschung durchführen würden, vor. Sowohl schriftlich als auch mündlich wurden Kinder und Eltern darüber aufgeklärt, dass sie jederzeit ihre Mitarbeit im Projekt abbrechen könnten, auch wenn sie zuvor zugesagt hatten. Zudem wurden sie darüber informiert, dass sämtliche Daten pseudonymisiert würden. Zur eigenen Absicherung der Forscherinnen wurde schriftlich und im Gespräch auf die Grenzen der Forschung hingewiesen, wie zum Beispiel keinen Einfluss auf das Asylverfahren oder die Wohnsituation direkt ausüben zu können (siehe den Beitrag von Trần).

Für die vorliegende Studie waren die Identifizierung von Schlüsselpersonen sowie die Entwicklung konkreter unterkunftsbezogener Fragestellungen von der Dynamik im Feld geleitet, nach dem Prinzip „research follows actor“. Nach Niederschrift und erster Dateninterpretation trafen die Forschenden für jede Unterkunft eine Auswahl möglicher teilnehmender Kinder und ihrer Eltern. In Anlehnung an das ökologische Modell von Uri Bronfenbrenner (vgl. Bronfenbrenner 1979) würde vom einzelnen Kind auszugehen und von diesem weitere Kreise zu ziehen sein, so dass Verschränkungen von Mikro-, Mesound Makroebene untersucht werden konnten: Das Datenmaterial der Studie umfasst die Perspektiven von Kindern, Eltern, Heimleitungen, Sozialarbeiter*innen und Kinderbetreuer*innen, dem Sicherheitsdienst und anderen Personen wie technischer Hilfskräfte und Stadtteilmüttern. In der Studie wurden 
die interaktionale Beziehungsebene mit den wichtigsten Bezugspersonen der Kinder (wie Familie, Peers und Mitarbeiter*innen), institutionelle Abläufe und Routinen (Räume und Bewegungen von Kindern), organisationale Vorgaben in den Unterkünften, Diskurse und Rechtsvorschriften, die das Leben geflüchteter Kinder strukturell rahmen, analysiert.

\subsection{Datenerhebung und Dateninterpretation}

Die Forscherinnen setzten akteur*innenzentrierte Methoden in der Zusammenarbeit mit Kindern und anderen Feldakteur*innen wie Mitarbeiter*innen und Bewohner*innen ein. Zur Generierung von Daten wurden neben teilnehmenden Beobachtungen (vgl. Flick 1999), ero-epische Gespräche (vgl. Girtler nach Halbmayer \& Salat 2011) und leitfadengestützte Expert*inneninterviews mit Kindern und Eltern geführt (vgl. Christensen \& Prout 2002: 480; White \& Bushin 2011). Auch nonsprachliche Methoden wurden verwendet (wie bildbasierte und raumorientierte partizipative Methoden). Zur Unterstützung der Kommunikation insbesondere während der Anfangsphase im Feld standen Sprachmittler*innen (für Farsi, Arabisch und Kurdisch) bereit bzw. wurde auf Englisch als gemeinsame Sprache ausgewichen. Das Datenmaterial umfasst teils audiotranskribierte leitfadengestützte Interviews, teils Feldprotokolle aus der teilnehmenden Beobachtung

Der Einsatz besonderer kindzentrierter bzw. nichtschriftbasierter Methoden sollte dazu beitragen, dass insbesondere sehr junge Kinder sich dazu eingeladen fühlten, eigene Ideen, Interessen, ihr Können und ihre Fertigkeiten in den Forschungsprozess einzubringen, ohne sich einem starren Forschungskorsett unterwerfen zu müssen (vgl. Gallacher \& Gallagher 2008; White 2011). Nach White (2011) kann der Einsatz kindzentrierter Methoden dazu beitragen, dass sich gute, das heißt vertrauensvolle Arbeitsbeziehungen mit Kindern, Mitarbeiter*innen und Familien in den Unterkünften entwickeln. Eine derart fundierte Arbeitsbeziehung zwischen den Forschungsbeteiligten ist besonders bedeutsam im Kontext von Flucht und mit den damit zusammenhängenden Faktoren wie Unterbringungsverhältnissen, rechtlichen Unsicherheiten und der allgemein prekären, wenn nicht sogar bedrohlichen Situationen für Kinder (vgl. White 2011; auch White \& Busin 2011). In der Studie eigneten sich die Kinder situationsspezifisch Forschungsinstrumente der Forscherinnen an und ergriffen teilweise Besitz von diesen selbst: Die Kinder umklammerten beispielweise die Beine einer Forscherin und forderten diese auf mitzuspielen (siehe die Beiträge von Fichtner \& Trần, Scott \& Le und Scott). Nach der Auswertung wurden die Erkenntnisse der Dateninterpretation an Eltern und Einrichtungsleitungen anonymisiert und verfremdet rückgespiegelt und zur Diskussion gestellt. Auch dies wurde dokumentiert und fand Eingang in die 
abschließende Interpretation. Zum Dank für ihre Mitarbeit im Forschungsprojekt erhielten die Kinder einen Gutschein.

In Anlehnung an die Grounded Theory erfolgte die Datenerhebung und Datenauswertung in einem wechselseitigen Prozess, der zirkulär oder wellenförmig vorstellbar ist (vgl. Strübing 2002; Breidenstein et al. 2013). Beobachtungen und Interviews sowie informelle Gespräche wurden zeitnah zur Erhebung transkribiert und von der Forschenden bzw. dem Forschungstandem interpretiert. Über das Vorgehen des offenen, axialen und selektiven Kodierens (vgl. Strauss \& Corbin 1996; Breuer et al. 2018), durch die Einnahme einer kontrastierenden Perspektive (vgl. Bollig et al. 2016) im Sinne konstruktivistischer Grounded Theory (vgl. Charmaz 2014) und der Situationsanalyse nach Clarke (2012, 2003) wurden die Daten interpretiert, weiterführende Fragen entwickelt und diese an das bereits erhobene Datenmaterial (erneut) gestellt. Die aus der Dateninterpretation gewonnenen Erkenntnisse konnten im Feld überprüft bzw. durch fokussierte Beobachtungen und erneute Gespräche vertieft werden und damit zu einer sowohl gegenstandsbezogenen als auch zu einer nächst abstrakteren Erkenntnisebene vordringen. Die in unterschiedlichen Unterkünften erhobenen Daten wurden entlang der spezifischen Fragestellung der Gewalterfahrungen miteinander vergleichend analysiert (siehe den Beitrag von SchulzAlgie). ${ }^{6}$

Über die Dauer von acht Monaten konnten die Ethnografinnen in den Unterkünften Prozesse und Entwicklungen beobachten sowie Handlungs- und Organisationsstrukturen aufdecken. Neben der Beobachtung von Sprachentwicklungen und der Beteiligung an der Gestaltung von Beziehungen wurde beobachtbar, dass und wie die wahrgenommene infrastrukturelle Ressourcenknappheit der Einrichtungen (materiell, räumlich, personell, finanziell) eingebettet ist in Ungewissheit und Unsicherheit, die das Feld als solches durchziehen: rechtlich in Bezug auf die Anerkennung des Aufenthaltsstatus der Bewohner*innen sowie in Bezug auf ihre Verweildauer in den Unterkünften; in Bezug auf den Fortbestand der jeweiligen Unterkunft; in Bezug auf die Arbeitsplatzgarantie der Beschäftigten und soziostrukturell in Bezug auf die Befugnisse der Mitarbeiter*innen; in Bezug auf das Zusammenleben mit fremden Menschen ohne gemeinsame Sprache und in Bezug auf die Kontakte außerhalb der Unterkunft. Aus der Dateninterpretation wurde zur Beschreibung der Beziehungsverhältnisse in den Unterkünften der Begriff Rollenkonfusion konzeptionell entwickelt. Darunter sind Rollenverunsicherungen und Grenzüberschreitungen gefasst, die in allen Beziehungsverhältnissen im Feld für die Forscherinnen beobachtbar und erfahrbar waren. In der Studie wurde als zentrale Kategorie die Kategorie Raum entwickelt. Dabei wird Raum als Produkt sozialer Prozesse identifiziert (vgl. Levebre 1991; siehe den Beitrag von Scott \& Le). Als „Beziehungsraum“, „Bewegungsraum“ und „Handlungsraum“ wurden

6 Zum konkreten Vorgehen der Datenanalyse entlang spezifischer Fragestellungen siehe die Beiträge von Fichtner \& Trần und von Scott \& Le) 
drei grundlegende Bedeutungsvariationen herausgearbeitet (siehe den Beitrag von Fichtner \& Trần). Die Strukturiertheit sozialer Prozesse sowie ihre Materialisierung in diesen spezifischen Kontexten konnten als „,gewaltförmig“ und „diskriminierend“ identifiziert werden (siehe die Beiträge von Schulz-Algie und von Wihstutz „Mittendrin und außen vor").

Eine Auswahl an Besonderheiten und Herausforderungen, mit denen das Forscherinnenteam in der Laufzeit des Projekts konfrontiert war, werden im Folgenden abschließend kurz skizziert.

\section{Feldbezogene Herausforderungen der empirischen Forschung}

Die politischen Verhältnisse und die gestiegene mediale Aufmerksamkeit in den Jahren 2015/2016 führten zu einem gewachsenen Interesse an direkten Kontakten für Gespräche mit Geflüchteten in Unterkünften. Der Zugang zu potentiellen Forschungsteilnehmer*innen gestaltete sich als unerwartet zeitintensiv. Im Projekt wurde nach Adressen von Unterkünften über Zeitungsberichte recherchiert, es wurden Netzwerke zur Unterstützung von Geflüchteten kontaktiert und persönliche Beziehungen genutzt. Die staatlich veranlasste Unterbringung Geflüchteter in speziellen Unterkunftsformen führte dazu, dass Anfragen nach direkten Kontakten zu Geflüchteten bei der Heimleitung kanalisiert wurden. Neben Interessebekundungen wurde von Heimleitungen und Mitarbeiter*innen eine Vielfalt an Gründen für die Ablehnung des Forschungsanliegens angeführt: Diese reichten von logistischen Problemen wie der bevorstehenden Auflösung der Unterkunft über die plötzliche Verlegung aller Familien mit Kindern im interessierenden Alter bis hin zu allgemeinen Vorbehalten gegenüber Forschung oder zu Bedenken bezüglich der Forschungsbeteiligung von Kindern im Besonderen. Personalwechsel in Einrichtungen hatte weitere Verzögerungen oder Absagen zur Folge. So konnte das ursprüngliche Vorhaben, die Forschung in Unterkünften mit einer ausgewogenen Trägervielfalt durchzuführen, nur bedingt umgesetzt werden. Über die Schneeballmethode konnten schließlich Kinder und Familien in drei Unterkünften für die Forschung gewonnen werden. Unser Sample umfasst einschränkend ausschließlich Kinder und Familien aus Einrichtungen von Trägern, die dem Forschungsanliegen offen gegenüber gestimmt waren, damit wurden die Betreiber von Unterkünften, Heimleitungen und Mitarbeiter*innen zu Gatekeepern im Verhältnis zwischen unserem Forschungsanliegen und den Geflüchteten. Vor diesem Hintergrund ist grundsätzlich die Frage zu stellen, wer in Forschung repräsentiert wird. Wessen Stimme wird gehört und wessen Perspektiven erhoben? Wer setzt die Agenda, die interessierenden Themen der Forschung? Im 
Rahmen der Studie setzten sich die Forscherinnen mit diesen und anderen Fragen in Bezug auf gesellschaftliche Machtverhältnisse, in die die Forschung eingelassen ist, auseinander (siehe den Beitrag von Trần). Die Forschung mit Geflüchteten ist mit ähnlichen Dilemmata konfrontiert wie andere Vorhaben, die den Kontakt zu Menschen in Krankenhäusern, Gefängnissen oder Schulen, also Menschen in institutioneller Obhut suchen (vgl. Goffman 1973). Deren Sonderunterbringung bzw. Schutzbedürftigkeit kann zu einem Mittel ihrer Unterdrückung werden, wenn dadurch ihre gesellschaftliche Beteiligung in für sie bedeutsamen Bereichen eingeschränkt oder unterbunden wird. Kinder sind in dieser Situation in doppelter Weise schwer zu erreichen: Ihr rechtlicher Status macht die Beteiligung von Kindern an einer Forschung von der Zustimmung ihrer Eltern abhängig. Die Forschung mit Kindern in Unterkünften für Geflüchtete ist darüber hinaus von der Zustimmung anderer Gatekeeper wie den Trägereinrichtungen, Heimleitungen und Mitarbeiter*innen der Unterkünfte abhängig. Aus kinderrechtlicher Perspektive ist jedoch die informierte Einwilligung des Kindes zur Forschungsbeteiligung ebenso bedeutsam.

Im Verlauf des ethnografischen Forschungsprozess zeigte sich, dass und wie die Forschung selbst eine Intervention im Feld darstellt (vgl. auch Breidenstein et al. 2013; Flick 1999). Handlungs- und Situationslogiken im Feld forderten oftmals dazu auf, Position zu beziehen (siehe den Beitrag von Trần). Die Rolle der Ethnografin bedurfte der wiederholten Verhandlung und Klärung im Feld sowie es auch der Überprüfung und Reflexion des eigenen Rollenverständnis in Supervisionssitzungen und im gemeinsamen Austausch mit dem Forschungsteam erforderte ${ }^{7}$ (siehe die Beiträge von Trần und Scott). Insbesondere Missverständnisse und unbeabsichtigte oder auch unbemerkte Verletzungen in der Kommunikationsstruktur galt es als solche zu erkennen und zu klären, da sie die Forschungsbeziehung beeinträchtigen bzw. auch zu ihrem Abbruch führen konnten. Trotz bester Absichten und gewissenhafter Forschung wurden schließlich Daten aus dem Forschungsprojekt für die Veröffentlichung gesperrt. Davon betroffen waren insbesondere Erhebungsdaten mit dem Fokus auf Mitarbeitende in Einrichtungen.

Aus ethischer Perspektive wurden weiterführende Fragen aufgeworfen, ohne diesen jedoch im Rahmen der vorliegenden Studie explizit nachgehen zu können. Diese umfassen zum Beispiel die Informations- und Mitbestimmungskultur in Unterkünften und die Konstitutionsbedingungen des Verhältnisses zwischen Bewohner*innen und Mitarbeiter*innen sowie die Frage nach (externer) Kontrolle einrichtungsinterner Abläufe. Weitestgehend unbestimmt blieb auch die Frage nach dem Charakter oder Typ der Einrichtungen (vgl. Agamben 2002; Goffman 1973; Foucault 2005), um die es sich bei der Sam-

In diesem Zusammenhang sei darauf hingewiesen, dass die Zusammensetzung des Forschungsteams ausschließlich aus Frauen nicht beabsichtigt war. Eine kritische Reflexion dieses Umstandes wurde im Rahmen der Studie nicht vorgenommen. 
melunterbringung von Geflüchteten handelt. ${ }^{8}$ Andere Themen wie zum Beispiel die Rolle von Ehrenamtlichen (vgl. Karakayali 2018; Hamann et al. 2016) werden in der Studie nicht bearbeitet, weil sie aus der Fokussierung auf das einzelne Kind und seinem Alltag nicht zum relevanten Forschungsgegenstand wurden. Nicht ausreichend vertiefend analysiert werden konnte darüber hinaus das Verhältnis zwischen Sicherheitsschutz und anderen nichtpädagogischen Mitarbeiter*innen mit Kindern in den Unterkünften. Darüber hinaus könnte eine intersektional angelegte Untersuchung der Beziehungen unterschiedlicher Mitarbeiter*innengruppen mit geflüchteten Bewohner*innen Schlaglichter auf die Verstrickungen der Einflussfaktoren wie Nationalität, Sprache, Geschlecht und Alter auf die Beschäftigungspolitik und das berufliche Selbstverständnis in der Zusammenarbeit mit Geflüchteten werfen sowie Erkenntnisse über eingelagerte hierarchische Strukturen in diesen Verhältnissen liefern. Eine Analyse aus diskriminierungstheoretischer rassismuskritischer Perspektive steht ebenfalls aus.

\section{Literatur}

Amann, Klaus \& Hirschauer, Stefan (1997): Die Befremdung der eigenen Kultur. Ein Programm. In: Hirschauer, Stefan \& Amann, Klaus (Hrsg.): Die Befremdung der eigenen Kultur. Zur ethnographischen Herausforderung soziologischer Empirie. Frankfurt a. M.: Suhrkamp, S. 7-53.

Bamler, Vera; Werner, Jillian; Wustmann, Cornelia (2010): Lehrbuch Kindheitsforschung. Grundlagen, Zugänge und Methoden. Weinheim/München: Juventa.

Bollig, Sabine, Honig, Michael-Sebastian \& Nienhaus, Sylvia (2016): Vielfalt betreuter Kindheiten. Ethnographische Fallstudien zu den Bildungs- und Betreuungsarrangements 2-4jähriger Kinder. Belval: Université de Luxembourg.

Bourdieu, Pierre \& Wacquant, Loic J. D. (1996): Reflexive Anthropologie. Frankfurt a. M.: Suhrkamp.

Breuer, Franz, Muckel, Petra \& Dieris, Barbara (2018): Reflexive Grounded Theory. Eine Einführung in die Forschungspraxis. 3. vollständig überarbeitete und erweiterte Auflage. Wiesbaden: Springer.

Breidenstein, Georg, Hirschauer, Stefan, Kalthoff, Herbert \& Nieswand, Boris (2013): Ethnografie: Die Praxis der Feldforschung. Konstanz/München: UVK.

8 Eine soziologische Analyse der Unterbringung von Geflüchteten und ihrer Raumaneignungsstrategien wird von Schäfer zur Zeit durchgeführt (vgl. Schäfer 2015; im Zusammenhang mit der aktuellen Frage der Unterbringung Geflüchteter in Deutschland entstehen weitere soziologisch verortete Arbeiten von Bochmann, Annett; Susanne Becker, Christoph Mautz, Anne-Marlen Engler, vgl. DGS 2016). 
Bronfenbrenner, Uri (1979): The Ecology of Human Development: Experiments by Nature and Design. Cambridge, MA: Harvard University Press.

Bühler-Niederberger, Doris (2005): Kindheit und die Ordnung der Verhältnisse. Von der gesellschaftlichen Macht der Unschuld und dem kreativen Individuum. Weinheim/München: Juventa.

Charmaz, Kathy (2014): Constructing Grounded Theory. Second Edition. Los Angeles: Sage.

Christensen, Pia \& Prout, Alan (2002): Working With Ethical Symmetry in Social Research with Children. In: Childhood 9 (4), S. 477-497.

Clark, Alison \& Moss, Peter (2011): Listening to Young Children. The Mosaic Approach. London: National Children's Bureau.

Clarke, Adele (2012): Situationsanalyse- Grounded Theory nach dem Postmodern Turn. Herausgegeben und mit einem Vorwort von Reiner Keller. Wiesbaden: VS Springer.

Clarke, Adele (2003): Situational Analyses: Grounded Theory mapping after the postmodern turn. In: Symbolic Interaction, 26c(4), S. 553-576.

Cockburn, Tom (2007): Reconstructing Children's Agency: Boundaries of Rights and Care. In: Clare Beckett, Owen Heathcote, Marie Macey (Hrsg.): Negotiating Boundaries: Identities, Sexualities, Diversities. Newcastle: Cambridge Scholars Publishing, S. 155-166.

DGS (2016): Geschlossene Gesellschaft. 38. Kongress der Deutschen Gesellschaft für Soziologie. 26. -30. September 2016 Universität Bamberg, https://kongress 2016.soziologie.de/fileadmin/user_upload/DGS_Redaktion_BE_FM/Kongresse/Kongress_2016/dgs2016_Bamberg_Hauptprogramm-Zugriff.pdf (Zugriff 11.10.2018).

Emirbayer, Mustafa \& Mische, Ann (1998): What is Agency? In: American Journal of Sociology 104, S. 962-1023.

Flick, Uwe (1999): Qualitative Forschung. Theorie, Methoden, Anwendung in Psychologie und Sozialwissenschaften. 4. Auflage, Reinbek bei Hamburg: Rowohlt.

Foucault, Michel (2005): Wahnsinn und Gesellschaft. Eine Geschichte des Wahns im Zeitalter der Vernunft. Frankfurt a. M.: Suhrkamp.

Gandini, Lella (2011): Play and the Hundred Languages of Children. An Interview with Lella Gandini. In: American Journal of Play volume 4, number 1, summer 2011 , o.S. http://www.journalofplay.org/issues/4/1/interview/play-and-hundred-languages-children-interview-lella-gandini . (Zugriff 28.1.2019).

Giner, Clotilde (2007): The politics of childhood and asylum in the UK. In: Children \& Society 21, S. 249-260.

Goffman, Erving (1973): Asyle. Über die soziale Situation psychiatrischer Patienten und anderer Insassen. Frankfurt a. M.: Suhrkamp.

Goffman, Erving (1993): Rahmen-Analyse. Ein Versuch über die Organisation von Alltagserfahrungen. Übersetzung von Hermann Vetter. 3. Auflage, Frankfurt a. M.: Suhrkamp.

Halbmayer, Ernst \& Salat, Jana (2011) Qualitative Methoden der Kultur- und Sozialanthropologie. Das ero-eptische Gespräch. Institut für Kultur- und 
Sozialanthropologie, Universität Wien. https://www.univie.ac.at/ksa/elearning/cp/qualitative/qualitative-42.html (Zugriff 27.1.2019)

Hamann, Ulrike, Karakayli, Serhat, Wallis, Mira \& Höfler, Leif Jannis (2016): Koordinationsmodelle und Herausforderungen ehrenamtlicher Flüchtlingshilfe in den Kommunen. Qualitative Studie des Berliner Instituts für empirische Integrations- und Migrationsforschung, hrsg. von der Bertelsmann Stiftung.

Hungerland, Beatrice \& Kelle, Helga (2014): Kinder als Akteure - Agency und Kindheit. Einführung in den Themenschwerpunkt. In: ZSE Zeitschrift für Soziologie der Erziehung und Sozialisation 34 (3), S. 227-232.

James, Allison, Jenks, Chris \& Prout, Alan (1998): Theorizing Childhood. Cambridge: Polity Press.

Karakayali, Serhat (2018): Ehrenamtliches Engagement für Geflüchtete in Deutschland. Ehrenamtliches Engagement für Geflüchtete in Deutschland. Unter Mitarbeit von Mareike Heller. State-of-Research Papier 09, Verbundprojekt ,Flucht: Forschung und Transfer', Osnabrück: Institut für Migrationsforschung und Interkulturelle Studien (IMIS) der Universität Osnabrück / Bonn: Internationales Konversionszentrum Bonn (BICC).

Kaukko, Mervi, Dunwoodie, Karen \& Riggs, Elisha (2017): Rethinking the Ethical and Methodological Dimensions of research with Refugee Children. In: Zeitschrift für internationale Bildungsforschung und Entwicklungspädagogik ZEP 1, S. 16-21.

Lewek, Mirjam \& Naber, Adam (2017): Kindheit im Wartezustand. Studie zur Situation von Kindern und Jugendlichen in Flüchtlingsunterkünften in Deutschland.Köln: UNICEF.

Maragel, Michel \& Manachi, Sandra (2018): The resilience of Syrian refugee children in Lebanon. In: Pace, Michelle \& Sen, Somdeep (Hrsg.): Syrian refugee children in the middle east and europe. Integrating the yound and exiled. London: Routledge, S. 32-45.

Niedrig, Heike \& Seukwa, Henri (2010): Die Ordnung des Diskurses in der Flüchtlingskonstruktion: eine postkoloniale Re-Lektüre. In: Diskurs Kindheits- und Jugendforschung 5 (2), S. 181-193, http://www.budrich-journals.de/index.php/ diskurs/article/view/3793 (Zugriff 11.5.2018).

Pupavac, Vanessa (2001): Misanthrophy without borders: The international Children's Rights Regime. In: Disasters 25 (2). S. 95-112.

Pupavac, Vanessa (2008): Refugee Advocacy, Traumatic Representations and Political Disenchantment. In: Government and Opposition 43 (2), S. 270-292.

Schäfer, Philipp (2015): Das Flüchtlingswohnheim. Raumcharakter und Raumpraxis in der Gemeinschaftsunterkunft. In: sinn-provinz. Kultur-soziologische working papers, Nr. 7, hrsg. von der Universität Leipzig, www.sinnprovinz.unileipzig.de (Zugriff 13.8.2018).

Scherr, Albert (2012): Soziale Bedingungen von Agency. Soziologische Eingrenzungen einer sozialtheoretisch nicht auflösbaren Paradoxie. In: Bethmann, Stephanie, Helfferich, Cornelia, Hoffmann, Heiko \& Niermann, Debora (Hrsg.): Agency. Qualitative Rekonstruktionen und gesellschaftstheoretische Bezüge von Handlungsmächtigkeit. Weinheim/Basel: Beltz Juventa, S. 99-121. 
Seeberg, Marie Louise, Bagge, Cecilie \& Enger \& Tuls André (2009): No Place. Small children in Norwegian asylum-seeker reception centres. In: Childhood 16 (3), S. 395-411.

Strübing, Jörg (2002): Just do it? Zum Konzept der Herstellung und Sicherung von Qualität in grounded theory-basierten Forschungsarbeiten. In: Kölner Zeitschrift für Soziologie und Sozialpsychologie 54 (2), S. 318-342.

Vitus, Kathrine (2010): Waiting Time. The de-subjectification of children in Danish asylum centres. In: Childhood 17 (1), S. 26-42.

Voß, Günter G (2000): Alltag: Annäherungen an eine diffuse Kategorie. In: Klaus Boehnke, G. Günter Voß, Werner Holly, (Hrsg.): Neue Medien im Alltag. Begriffsbestimmungen eines interdisziplinären Forschungsfeldes. Wiesbaden: Springer VS, S. 31-77.

Wells, Karen (2009): Childhood in a Global Perspective. Cambridge \& Malden, MA: Polity Press.

White, Allen (2012): ,Every Wednesday I am happy'. Childhoods in an Irish Asylum Centre. Population, Space and place 18, S. 314-326.

White, Allen \& Bushin, Naomi (2011): More than methods: learning from research with children seeking asylum in Ireland. In: Population, Space and Place 17 (4), S. 326-337.

Wihstutz, Anne (2018) Grundbegriff Kindheit. Eine soziologische Perspektive. In: Kleeberg-Niepage, Andrea \& Rademacher, Sandra (Hrsg.): Kindheits- und Jugendforschung in der Kritik. (Inter-)Disziplinäre Perspektiven auf zentrale Begriffe und Konzepte. Wiesbaden: Springer, S. 91-110. 


\section{Mittendrin und außen vor - Geflüchtete Kinder und die Umsetzung von Kinderrechten in Deutschland}

Anne Wihstutz

„At the heart of the discourse and practice of children's rights lie basic assumptions about the relationship between the young and the nation-state []. Yet, for young people displaced across national borders by armed conflict and political oppression the connections to sovereign states is often far from natural or automatic" (Boyden \& Hart 2007: 237).

\section{Einleitung}

Kinder, die vor Gewalt, Konflikten und anderen ihr Leben bedrohenden Verhältnissen fliehen und Schutz in einem anderen Land suchen, sind darauf angewiesen, in diesem Land Sicherheit zu finden und ihre Bedürfnisse befriedigen zu können.

Minderjährige begleitete geflüchtete Kinder, die Asyl begehren, finden sich inmitten eines rechtlichen Spannungsverhältnisses wieder zwischen national bestimmten Migrations- und Asylrechten und den ,universellen“ Rechten des Kindes der UN-Kinderrechtskonvention. Je nachdem, unter welcher politischen Kategorie asylsuchende Kinder verhandelt werden, unterscheiden sich die politischen und praktischen Folgen für Kinder sehr. Werden sie als „Kinder" diskursiv verhandelt, ist der politisch-rechtliche Bezugsrahmen die UNKinderrechtskonvention bzw. die landes- und kommunalpolitisch verfassten Rechte von Kindern und Jugendlichen wie das Sozialgesetzbuch VIII, Kinderund Jugendhilfe (SGB VIII) in Deutschland. Werden sie hingegen vorrangig als „Asylsuchende“ definiert, werden sie zum Objekt nationalstaatlich orientierter Migrations- und Asylpolitik. In jedem Fall wird ein spezifisches formales Rechtsverhältnis zwischen Staat und dem einzelnen Kind bzw. der spezifischen Gruppe „begleiteter minderjähriger Flüchtlinge“ konstituiert. Mit der Unterzeichnung der UN-Kinderrechtskonvention und der Rücknahme des Vorbehalts gegenüber ausländischen Kindern hat sich die Bundesregierung 
verpflichtet, die Prinzipien und Artikel der Konvention in nationales Recht vorbehaltlos umzusetzen.

Gegenstand dieses Beitrags ist die Frage, welchen Status diese Rechte im Verhältnis zum Prinzip nationaler Souveränität und damit zu einer nationalstaatlich orientierten Asyl- und Migrationspolitik einnehmen. Der Beitrag beleuchtet ambivalente und widersprüchliche Folgen der Bezugnahme auf konfligierende Rechtsdiskurse in Politik und Praxis für die Lebensverhältnisse geflüchteter Kinder. Dabei gehe ich wie folgt vor: im ersten Kapitel werden die allgemeinen asylrechtlichen Bestimmungen zum Zeitpunkt der Durchführung der Studie skizziert. Im zweiten Kapitel werfe ich Schlaglichter auf ausgewählte Bereiche der Lebensverhältnisse asylbegehrender Kinder in Deutschland und diskutiere diese mit Bezug auf die Rechte von Kindern in der UN Kinderrechtskonvention sowie mit Bezug auf relevante aufenthalts- und asylrechtliche Grundlagen. Zur theoretischen Rahmung der Diskussion wird im dritten Kapitel das Konzept der diskursiven Hegemonie nach Mouffe und Laclau (1985) knapp erläutert und schließlich werden im vierten Kapitel diskursive bzw. rechtliche Widersprüche in der Zuweisung der politischen Identität von asylbegehrenden Kindern als „Kinder“ bzw. als „Flüchtlinge“ mit Bezug auf Deutschland und andere ausgewählte europäische Staaten betrachtet. Die Ausführungen zu den rechtlichen Grundlagen beziehen sich, wenn nicht anders ausgewiesen, auf den Zeitraum Mai 2016 bis Februar $2017 .{ }^{1}$

\section{Asylpolitischer Rahmen in Deutschland: system(at)ische Unsicherheiten?!}

Im Zusammenhang mit der schnell anwachsenden Zahl schutz- und asylsuchender Menschen in Deutschland, insbesondere im Jahr 2015, wurden das Asylrecht auf nationaler Ebene und asylrechtliche Verordnungen auf EUEbene mehrmals novelliert. ${ }^{2}$ Die völkerrechtliche Grundlage für länderüberquerende Schutzsuchende ist die Genfer Flüchtlingskonvention. Diese garantiert Geflüchteten grundlegende Rechte im Aufnahmeland (vgl. Genfer Flüchtlingskonvention 1951). Wer Flüchtling ist, darf hiernach nicht in ein Land

1 In diesem Beitrag stehen nicht die handelnden Akteur*innen selbst, also die Kinder und ihre Eltern im Fokus. Eine akteurzentrierte Perspektive nehmen die Beiträge von Scott \& Le, Fichtner \& Trần und Schulz-Algie ein.

2 Die rechtliche und soziale Situation geflüchteter Kinder in Deutschland ist im Zusammenhang mit der Asylpolitik in Europa zu diskutieren. Das Gemeinsame Europäische Asylsystem steht auch aktuell wieder im Zentrum europäischer Reformen (siehe auch Stellungnahme Bundesverband der unbegleiteten Minderjährigen Flüchtlinge (BumF) zur Reform des Gemeinsamen Europäischen Asylsystems, GEAS 2017). 
abgeschoben werden, in dem ihr oder ihm Verfolgung droht (vgl. Boyden \& Hart 2007). ${ }^{3}$ Während die Genfer Flüchtlingskonvention von 1951 noch stark durch die Erfahrungen des Holocaust und des Zweiten Weltkriegs geprägt ist, dominiert im asylpolitischen Diskurs - spätestens seit dem Fall des Eisernen Vorhangs - seit den 1990er Jahren die politische Figur des ,illegitimate asylum-seeker" (Vitus \& Lidén 2010: 65). Das heißt, im politischen Diskurs wird zunehmend unterschieden zwischen schützenswerten und sogenannten rechtswidrigen Asylsuchenden.

Die im Folgenden skizzierten Rahmenbedingungen und asylrechtlichen Veränderungen sind allgemein zu verstehen. Auf die besondere Situation von Kindern wird in einem späteren Kapitel eingegangen.

Nach ihrer Erstregistrierung erhalten Asylbewerber*innen in Deutschland nach dem Asylbewerberleistungsgesetz (AsylbLG) seit März 2015 vorrangig Sachleistungen in Erstaufnahmeeinrichtungen in Form von Essenspaketen, Kleidung und Möbel bzw. Gutscheine zur Sicherung des Grundbedarfs. „Zur Deckung persönlicher Bedürfnisse des täglichen Lebens" steht ihnen ein geringes Bargeld zur Verfügung. Für Kinder im Alter unter sechs Jahren sind monatlich 79 Euro vorgesehen zur Deckung ihres täglichen Bedarfs (§3 AsylbLG). Asylbewerber*innen haben bei Leistungsbezug nach § 3 AsylbLG keinen Anspruch auf eine Krankenversicherung. Das Sozialamt ist für die Sicherstellung der medizinischen Versorgung zuständig. Nur bei akuter Erkrankung und bei Schmerzzuständen ist die erforderliche ärztliche und zahnärztliche Behandlung zu gewähren ( $\S 4$ und 6 AsylbLG) ${ }^{4}$ Dabei wird teilweise von Behördenmitarbeiter*innen und nicht etwa von Ärzt*innen entschieden, ob eine Erkrankung akut genug ist für die Ausstellung der erforderlichen Dokumente zur medizinischen Behandlung. Nach 15 Monaten Aufenthalt in Deutschland können Asylbewerber*innen seit März 2015 unter bestimmten Voraussetzungen gemäß § 2 AsylbLG Leistungen im Umfang der Sozialhilfe ${ }^{5}$

3 Diese Rechte gelten in den Ländern, die die Konvention unterschrieben und ratifiziert haben. Nicht alle Staaten haben die Konvention ratifiziert.

4 Ergänzend zum Asylbewerberleistungsgesetz regelt Artikel 19 EU-Aufnahmerichtlinie 2013/33 soziale und medizinische Mindeststandards für Asylsuchende (mit einer Aufenthaltsgestattung) wie auch die geeignete psychologische Betreuung (vgl. BAfF 2017); González Méndez de Vigo et al. 2017). Ich danke Nerea Gonzalez Mendez de Vigo für die wertvollen Hinweise zu den hier angeführten juristischen Rahmenbedingungen des nationalen und internationalen Asylrechts. Ihre Expertise hat maßgeblich dazu beigetragen, dass mir der komplexe juristische Sachverhalt verständlicher wurde. Sollten Aussagen fehlerhaft oder missverständlich sein, bin ausschließlich ich dafür verantwortlich.

5 Mit dem Gesetz zur Einführung beschleunigter Asylverfahren wurden die 2015 zuvor angehobenen Grundleistungsbeträge für Asylbewerber*innen erneut herabgesetzt. Der Sachverständigenrat für Migration berechnet für eine alleinstehende erwachsene Person, die nicht mehr in einer Erstaufnahmeeinrichtung wohnt, monatlich 354 Euro. Zum Vergleich: Der Arbeitslosengeld-II-Regelsatz, der laut Bundesverfassungsgericht als Existenzminimum gilt, liegt seit 1. Januar 2018 bei 416 Euro (vgl. SVR 2018: 7). 
und eine elektronische Gesundheitskarte beantragen. Im Gegenzug zu dieser als Erleichterung und Verbesserung wahrgenommenen Veränderung wurden der Zuzug und die Antragstellung auf Asyl in Deutschland erschwert.

Seit März 2015 gelten drei Balkanstaaten (Serbien, Bosnien und Mazedonien) als ,sichere Herkunftsstaaten“. Damit haben asyl- und schutzsuchende Menschen aus diesen Ländern kaum Chancen auf Anerkennung. Die Liste der sogenannten sicheren Herkunftsstaaten soll auf weitere Länder ausgeweitet werden. Die Chancen gesellschaftlicher Teilhabe für Asylbewerber*innen aus diesen sogenannten sicheren Herkunftsstaaten sind stark eingeschränkt bzw. ausgeschlossen. ${ }^{6}$ Das Asylverfahrensbeschleunigungsgesetz vom 24. Oktober 2015 führte zu weiteren Einschränkungen: Die Pflicht, in Erstaufnahmezentren $\mathrm{zu}$ wohnen, wurde von maximal drei auf sechs Monate ausgeweitet. ${ }^{7}$ Damit verknüpft ist die Beschränkung der Bewegungsfreiheit auf den zugewiesenen Landkreis (Residenzpflicht), das absolute Arbeitsverbot und das Verbot, eine eigene Wohnung zu beziehen. Statt wie bisher drei Monate auf Sachleistungen angewiesen zu sein, verlängert sich die Bezugszeit auf sechs Monate, was insbesondere für Kinder, Kranke, z. B. Allergiker*innen, und Schwangere zu Problemen führt. Nach dem AsylbLG ist der zustehende minimale Barbetrag für den persönlichen Bedarf in Erstaufnahmeeinrichtungen teilweise oder ganz durch Sachleistungen zu ersetzen. Diese Regelung kann auch auf Gemeinschaftsunterkünfte ausgedehnt werden. Nach der Unterbringung in Erstaufnahmezentren werden Schutzsuchende während des Asylverfahrens in Gemeinschaftsunterkünften untergebracht. Der Familiennachzug für Personen, denen subsidiärer Schutz ( 4 Abs. 1 AsylG) zuerkannt wurde, wurde mit der Gesetzesnovellierung für zunächst zwei Jahre ausgesetzt (s. u.). ${ }^{8}$

Personen, bei denen ein ,rechtmäßiger und dauerhafter Aufenthalt zu erwarten ist" (vgl. GGUA 2016: 2), sind in „Erstaufnahmeeinrichtungen plus“ (EAE + ) „mit deutlich erweitertem Funktionsumfang“ aufzunehmen (Senat Berlin 2016: 13). Demgegenüber sind Personen mit geringen Chancen auf

Nach $§ 45 a$ Abs. 2 AufenthG heißt es zum Beispiel, dass der Zugang zu neuen berufsbezogenen Deutsch-Sprachförderangeboten ,,ausgeschlossen [ist] für einen Ausländer, der eine Aufenthaltsgestattung nach dem Asylgesetz besitzt und bei dem ein dauerhafter und rechtmäßiger Aufenthalt nicht zu erwarten ist. Bei einem Asylbewerber, der aus einem sicheren Herkunftsstaat nach $\S 29$ a des Asylgesetzes stammt, wird vermutet, dass ein rechtmäßiger und dauerhafter Aufenthalt nicht zu erwarten ist" (GGUA 2016: 3).

7 Die neue Koalitionsregierung aus SPD, CDU und CSU hat im Koalitionsvertrag die Einrichtung von sogenannten AnKER-Zentren aufgenommen: Der Prozess der Erstregistrierung, der Asylprüfung und gegebenenfalls die Abschiebung sollen gebündelt in diesen Zentren abgewickelt werden. Die Verweildauer in diesen Zentren kann bis zu 24 Monaten umfassen (vgl. Koalitionsvertrag 2018).

8 Im Koalitionsvertrag wurde der Familiennachzug für subsidiär Geschützte mit Beginn 1. August 2018 auf 1000 Personen pro Monat eingeschränkt. Der Familiennachzug war ein Kernthema der Auseinandersetzung in den Koalitionsverhandlungen (vgl. Süddeutsche Zeitung, 18.1.2018; Pro Asyl 2015). 
Anerkennung ihres Schutzgesuches ( $§ 30 \mathrm{a}$ AsylG) seit Oktober 2015 in sogenannten Ausreisezentren ( $\$ 5$ Abs. 5 AsylG) unterzubringen. Die Entscheidung darüber ist Ländersache (vgl. ebd.). Die soziale, ökonomische und kulturelle Beteiligung der Menschen - wozu auch der Zugang zu Sprachkursen für Erwachsene und der Besuch von Kindertagesstätten für junge Kinder zählen wird dadurch erheblich eingeschränkt bzw. unmöglich. Neben den Verhältnissen der Unterbringung beeinträchtigt das Warten die psychische und physische Gesundheit der so Untergebrachten (vgl. Lewek \& Naber 2017; siehe auch Wihstutz „Zentrale Erkenntnisse und Empfehlungen“).

Nach dieser kurzen Skizze des allgemeinen asylpolitischen Rahmens wird im Folgenden auf die besondere Situation von Kindern in Deutschland eingegangen.

Ich analysiere die rechtliche Lage geflüchteter Kinder aus zweierlei Perspektive, die ihre politische Zuschreibung einmal als „Kinder“ und einmal als „Asylsuchende“ fokussiert. Als Ausgangslage wird auf die fehlende verfassungsrechtliche Verankerung von Kinderrechten in Deutschland verwiesen, anschließend werden Schlaglichter auf ausgewählte Lebensverhältnisse minderjähriger Geflüchteter und die Umsetzung ihrer Kinderrechte geworfen. In einem weiteren Schritt werden diese aus der Perspektive des Asylrechts knapp beleuchtet.

\section{Kinderrechte auf dem Vormarsch?!}

Im Zusammenhang mit (Bundestags-)Wahlen zeigen sich Politiker*innen gerne Kindern und ihren Rechten zugewandt. Vor der Bundestagswahl 2017 wurde wiederholt der Vorstoß unternommen, die Rechte von Kindern zu stärken. Bislang kommen Kinder im Grundgesetz ,lediglich als Objekte elterlicher Verantwortung vor", so die Direktorin des Deutschen Instituts für Menschenrechte, Beate Rudolf, in der Pressemitteilung des Instituts anlässlich des Internationalen Tags der Kinderrechte am 20. November 2016 in Berlin (Deutsche Liga für das Kind 2016: 68). „Die Zeit ist reif“, schreibt die Deutsche Liga Für Das Kind, dass 25 Jahre nach Inkrafttreten der UN-Kinderrechtskonvention in Deutschland die Kinderrechte im Grundgesetz verankert würden (Deutsche Liga für das Kind 2016: 68). ${ }^{9}$ Befürworter*innen der Aufnahme von Kinder-

9 Die Forderung, Kinderrechte ins Grundgesetz aufzunehmen, ist darauf gerichtet, Kindern in Deutschland in der Verfassung ein eigenes Grundrecht auf bestmögliche Förderung (Bildung) und ein gleiches Grundrecht auf Partizipation, also auf Teilnahme wie auch Anhörung in allen Entscheidungen, die sie selbst betreffen, aufzunehmen. Diese Forderung bezieht sich auf die in der UN-Kinderrechtskonvention verbrieften Rechte des Kindes (Artikel 3, 28, 12 KRK) (vgl. Peschel-Gutzeit 2015: 8). 
rechten ins Grundgesetz monieren, dass Kinder in der deutschen Verfassung nicht ausdrücklich als Rechtssubjekte benannt werden, sondern als Anhängsel ihrer Eltern. Mit der Aufnahme der Kinderrechte ins Grundgesetz werde ein Gegengewicht gegenüber dem elterlichen Erziehungsrecht geschaffen. Trotz der Anerkennung der eigenständigen Rechtsträgerschaft von Kindern durch das Bundesverfassungsgericht ${ }^{10}$ zeige sich dieses nicht durchgängig in der Rechtsprechung und Rechtswirklichkeit für Kinder. Es gelte, die Rechtsträgerschaft eines jeden Kindes anzuerkennen, um zentrale Rechte wie den Maßstab des Kindeswohls (oder ,the best interests of the child“, wie es im Englischen heißt), das Verbot der Diskriminierung, das Recht auf Entwicklung und das Recht auf Schutz vor Gewalt vorrangig bei allen Maßnahmen, die Kinder betreffen, zu berücksichtigen (Artikel 3 Satz 1 UNKRK).

Gegner*innen einer verfassungsrechtlichen Verankerung der Kinderrechte betonen, die bestehenden Rechte seien bisher nur unzureichend umgesetzt worden, denn das Grundgesetz umfasse jeden Menschen, egal wie alt er sei (vgl. Wapler 2015a). Dabei konstatiert Wapler, dass die Perspektive und die Willensäußerung von Kindern nur schrittweise entsprechend ihrer sich entwickelnden Autonomiefähigkeit zu berücksichtigen seien und nach geltendem Recht ,immer [...] unter dem Vorbehalt des ,objektiven Wohls““ stünden. ,[D]ie paternalistische Entscheidung Dritter über die Belange der Kinder [ist] der Regelfall“" (Wapler 2015b: 265). Kritisch merkt Liebel (2018) aus ethischer bzw. soziologischer Perspektive dazu an, dass es hinsichtlich des Gleichberechtigungsanspruchs von Kindern erforderlich sei ,zu fragen, wie [der Machtasymmetrie zwischen Erwachsenen und Kindern] entgegengewirkt werden könnte“, ohne sie als ,,anthropologische Konstante“ im Sinne eines ,behutsamen Paternalismus“ in rechtsethischen Diskursen zu verfestigen (Liebel 2018: 70).

Ich diskutiere im Folgenden, wie sich die fehlende verfassungsmäßige Verankerung der UN-Kinderrechte auf die Lebensverhältnisse der Kinder auswirkt, die mit ihren Eltern das Asylverfahren durchlaufen. Dabei werfe ich Schlaglichter auf ausgewählte Lebensbereiche der jungen Kinder, wie auf ihre Unterbringung und Versorgung sowie ihren Zugang $\mathrm{zu}$ frühkindlicher Bildung.

Staaten, die die UN-Kinderrechtskonvention ratifiziert haben, sind dazu verpflichtet, geeignete Gesetzgebungs- und Verwaltungsmaßnahmen sowie andere Maßnahmen zu ergreifen, um die in der Konvention enthaltenen Rechte umzusetzen (Artikel 4 UNKRK). Das bedeutet, dass Regierungen verantwortlich dafür sind, die Prinzipien und Bestimmungen der Kinderrechtskonvention in der nationalen Rechtsordnung anzuwenden und umzusetzen. In der Kinder-

10 Bereits im Jahr 1968 hatte das Bundesverfassungsgericht anerkannt, dass das Kind ,ein Wesen mit eigener Menschenwürde und einem eigenen Recht auf Entfaltung seiner Persönlichkeit im Sinne der Artikel 1 Abs. 1 und Artikel 2 Abs. 1 GG ist" (BVerfG, 29.07.1968- 1 BvL 20/63; 1 BvL 31/66; 1 BvL 5/67; vgl. Peschel-Gutzeit 2015: 8). 
rechtskonvention lassen sich drei Gruppen von Rechten unterscheiden, oftmals die drei Ps genannt (im Englischen ,participation“, ,protection“ und ,provision“" oder Beteiligung, Schutz und Förderung bzw. Versorgung). Aus dem Zusammenspiel von Artikel 12 UNKRK, dem Recht, gehört zu werden, und Artikel 3 Absatz 1 UNKRK geht hervor, dass nicht nur bei der Ermittlung des Kindeswohls die Sicht des Kindes eine große Rolle spielt, sondern auch die Beteiligung von Kindern als integraler Bestandteil zu verstehen ist, unter dem Begriff der Vorrangigkeit der,, best interests of the child" (vgl. General Comment Nr. 14 2013). Danach beschreibt Artikel 3 ein Recht, ein Prinzip und eine Vorgehensweise (vgl. Liebel 2017). Insofern stellt die UN-Kinderrechtskonvention für alle Kinder ein zentrales Dokument dar, das den Staat zur umfassenden Umsetzung ihrer Rechte verpflichtet. In diesem Sinne gelten Kinderrechte als universell.

\subsection{Widersprüchliche Praxis: mangelnde Kinderrechte von asylsuchenden Kindern bei Unterbringung, Versorgung und frühkindlicher Bildung}

Begleitete minderjährige geflüchtete Kinder werden mit ihren Eltern oder Sorgeberechtigten zunächst in Erstaufnahmeeinrichtungen untergebracht. Diese Institutionen liegen in der Verantwortung der Länder. ${ }^{11}$ Nach dem Wechsel in eine Gemeinschaftsunterkunft wechselt die Verantwortlichkeit in die Hände der Kommunalverwaltung. Eine Mitsprache, in welchem Bundesland oder an welchem Ort sie untergebracht werden, haben Kinder und Eltern nicht. Existierende Mindeststandards von Gemeinschaftsunterkünften betreffen überwiegend die Ausstattung und räumliche Beschaffenheit der Gebäude (vgl. Wendel 2014). Unterschiede gibt es in Bezug auf die Fläche, die für Kinder anzurechnen ist, die Sicherheitsvorkehrungen wie abschließbare Toiletten und Duschen, Kinderzimmer und Hausaufgabenzimmer.

Nach der EU-Richtlinie 2013/33 zur Aufnahme schutzsuchender Personen ist Minderjährigen in den Räumlichkeiten und Unterbringungszentren nach Artikel 23 Absatz 3 ,Gelegenheit zu Freizeitbeschäftigungen einschließlich altersgerechter Spiel- und Erholungsmöglichkeiten“ zu geben. Aktivitäten im Freien sollen möglich sein (vgl. Richtlinie 2013/33). ${ }^{12}$ Eine genauere Defini-

11 Familien mit minderjährigen Kindern unterstehen in Fragen der Unterbringung und Versorgung in primärer Verantwortung den nach Landesrecht für das Asylbewerberleistungsgesetz zuständigen Behörden, in der Regel sind das die Sozialämter.

12 Bis zum 20. Juli 2015 hätte Deutschland seine asyl- und aufenthaltsrechtlichen Gesetze den europäischen Vorgaben anpassen müssen. Da die Richtlinien nicht umgesetzt wurden, hat die im Aufnahmeland zuständige Behörde, in Deutschland das Bundesamt für 
tion fehlt. Andere Faktoren, die die Qualität des „Wohnens“ in den Unterkünften maßgeblich beeinflussen (können) - wie das Verhältnis zur Heimleitung und zu den Sozialarbeiter*innen - werden von den Mindeststandards nur indirekt erfasst, wie über den Betreuungsschlüssel und die Qualifikationsanforderungen an das Personal (vgl. Wendel 2014: 40). Eine an Standards orientierte Qualitätsanforderung für das Personal der Kinderbetreuung fehlt, auch der Bezug auf den Umgang mit dem Wachschutz bleibt vage. Im Unterschied zu anderen Einrichtungen, in denen sich Kinder für einige bis mehrere Stunden am Tag aufhalten, gilt für Unterkünfte nach dem Asylgesetz (§ 44 Abs. 3 AsylG) eine Betriebserlaubnis nach $\S 45$ SGB VIII nicht. Auch das Beschwerdemanagement, das im Jugendhilfebereich verpflichtend ist ( $\$ 8 \mathrm{~b}$ Abs. 2 Satz 2 SGB VIII; § 45 Abs. 2 Satz 3 SGB VIII), ist für diese Einrichtungen gesetzlich nicht vorgesehen.

In den Jahren 2015 und 2016 verbrachten Familien teilweise bis zu über zwölf Monaten in Notunterkünften wie Zelten und Turnhallen, da eine Unterbringung in Erstaufnahmeeinrichtungen und Gemeinschaftsunterkünften staatlicherseits nicht gewährleistet werden konnte (vgl. Lewek \& Naber 2017). Damit werden zentrale in der UN-Kinderrechtskonvention aufgeführten Rechte nicht umgesetzt: Insbesondere geht es dabei um das Recht auf Ruhe, Freizeit und Spiel und die Beteiligung an sozialem und kulturellem Leben (Artikel 31 UNKRK), das Recht auf Entwicklung (Artikel 6 Abs. 2 UNKRK), das Recht auf Bildung (Artikel 28 Abs. 1 UNKRK) und kulturelle Entfaltung (Artikel 29 Abs. 1 Nr. 1 UNKRK), das Recht auf die Pflege der kulturellen Identität, der Religion und die Verwendung der eigenen Sprache (Artikel 30 UNKRK).

Die Gewährung dieser Rechte ist für geflüchtete Kinder umso bedeutsamer, da sie auch in Erstaufnahmezentren und Gemeinschaftsunterkünften oftmals keinen eigenen Raum und keine Privatsphäre haben und die Unterkünfte schwerlich sichere Orte für Kinder darstellen (siehe die Beiträge von Fichtner \& Trần, Scott \& Le und von Schulz-Algie). Darüber hinaus sind die Unterkünfte oftmals sozialräumlich isoliert, insofern sie in Industriegebieten oder in anderen schwer zugänglichen Gegenden mit wenig soziokultureller oder verkehrstechnischer Infrastruktur liegen (vgl. DJI 2014).

Hinsichtlich des im Grundgesetz verankerten besonderen Schutzes der Familie (Artikel 6 GG) ruft die staatliche Unterbringungs- und Versorgungspraxis gegenüber asylsuchenden Kindern und ihrer Eltern Fragen bezüglich des leitenden Familienverständnisses auf. Nach geltendem Verfassungsrecht ist

\footnotetext{
Migration und Flüchtlinge (BAMF) am 20.7.2015 einen „Leitfaden zur unmittelbaren Anwendung" herausgegeben. Die EU-Aufnahmerichtlinie (Richtlinie 2013/33) und die EU-Verfahrensrichtlinie (VLR) bestimmen, dass in dem Aufnahmeland die zuständige Behörde, in Deutschland das BAMF, zu prüfen habe, ob die Asylantragsteller*innen gemäß Artikel 24 Abs. 1 VRL besondere Verfahrensgarantien benötigen. Für die Feststellung der besonderen Schutzbedürftigkeit nach Artikel 21 und 22 der Aufnahmerichtlinie sieht das BAMF hingegen die Landesbehörden in der Pflicht.
} 
die Pflege und Erziehung der Kinder das natürliche Recht der Eltern und die zuvörderst ihnen obliegende Pflicht (Artikel 6 GG). Erst bei Verletzung dieser Pflicht darf der Staat in Ausübung seines Wächteramts in das Elternrecht eingreifen. Wie asylsuchende Eltern ihrer Pflege- und Erziehungsverantwortung unter den gegebenen asylrechtlich bestimmten Verhältnissen überhaupt nachkommen können, bleibt hingegen offen.

In Anbetracht dieser Verhältnisse gewinnen die unterstützenden Funktionen der Leistungen nach dem Kinder- und Jugendhilfegesetz, SGB VIII, wie etwa die Angebote frühkindlicher Bildung, eine besondere Bedeutung. Der $\mathrm{Zu}-$ gang für asylsuchende Kinder zu Bildungseinrichtungen, Ruheräumen und Freizeitangeboten muss aus einer kinderrechtlichen Perspektive prioritär verfolgt werden. Derart wird ein elementarer Beitrag zur Verwirklichung der Grundbedürfnisse und von Kinderrechten auf Förderung, Erziehung, Bildung und Spielen geleistet (vgl. Meysen et al. 2016: 430). ${ }^{13}$ Obwohl die Datenlage der Länder unvollständig ist, lässt sie erkennen, dass nur eine Minderheit der Kinder aus Not- und Gemeinschaftsunterkünften und Erstaufnahmezentren frühkindliche Bildungsangebote wahrnimmt. ${ }^{14}$ Um überhaupt von diesen Möglichkeiten der Förderung sowie ihren Rechtsansprüchen zu erfahren, sind geflüchtete Eltern auf Unterstützung und Beratung durch kompetente Personen wie Sozialarbeiter*innen oder versierte ehrenamtliche Unterstützung angewiesen (vgl. DJI 2016).

Für Berlin hat die zuständige Senatsverwaltung unter anderem über das Landesprogramm Integrationslots*innen Beratung und Unterstützung von Geflüchteten im Masterplan Integration und Sicherheit (2015-2017) verankert. Zusätzlich zu vorhandenen Angeboten geben Integrationslots*innen (muttersprachliche) Orientierung bei Behördengängen, bei der Suche nach Sprachkursen und weiteren Beratungsangeboten. ${ }^{15}$

Berichte aus der Praxis sowie unsere Beobachtungen im Rahmen der Studie lassen darauf schließen, dass eine flächendeckende, strukturelle Verankerung ergänzender (muttersprachlicher) Beratungsangebote in den Unterkünften noch aussteht. Auch ist die Zusammenarbeit zwischen externen Einrich-

13 Für die Diskussion, ob sich der Geltungsbereich des SGB VIII auch auf geflüchtete Kinder und ihre Eltern erstreckt, vgl. Meysen et al. 2016.

14 Die Kommunen verfügen oftmals nicht über aktuelle und belastbare Statistiken über geflüchtete Kinder in Kindertagesstätten (vgl. für Berlin Fachgespräch 2017; für einen bundesweiten Überblick s. DJI 2016).

15 Im Rahmen des Masterplans wurden in Berlin in jedem Bezirk zusätzlich 0,5 Stellen der Erziehungs- und Familienberatung (EFB) als Leistung aus dem $§ 28$ SGB VIII für die aufsuchende Arbeit in den Unterkünften eingerichtet. Damit sollen Familien „im Prozess der Integration“ unterstützt und bei individuellen und familienbezogenen Problemen und Bedarfslagen identifiziert und Clearingprozesse angeregt sowie Betroffene zu Fachstellen weitervermittelt werden (vgl. Senatsverwaltung 2016: 19 f.). Der Bedarf wird als weitaus höher eingeschätzt als mit einer 0,5-Stelle pro Bezirk abzudecken wäre (vgl. Fachgespräch Juli 2017). 
tungen der frühkindlichen Bildung und Unterkünften nicht ausreichend über das Engagement einzelner Mitarbeiter*innen hinausgehend, gesichert. Ohne den Anspruch, repräsentative Aussagen zu treffen, deuten die Ergebnisse unserer Studie an, dass der Zugang zu frühkindlicher Bildung für geflüchtete Kinder sehr heterogen organisiert ist: von der täglichen, stundenweisen Betreuung in einer externen Kindertagesstätte, über eine unregelmäßige, stundenweise Betreuung durch sogenannte Kinderbetreuer*innen in den Unterkünften bis hin zum vollständigen Fehlen der Betreuung von Kindern in Unterkünften. $\mathrm{Zu}$ einer ähnlichen, die Unterbringung und den Zugang zu (frühkindlichen) Bildungseinrichtungen von geflüchteten Kindern in den Bundesländern umfassenden Einschätzung kommt die Bundesarbeitsgemeinschaft für Kinder- und Jugendschutz in ihrem Dossier „Geflüchtete Kinder“ (vgl. BAG 2016). Vielfach fehlen in den Unterkünften Kinderbetreuungsangebote ebenso wie $\mathrm{Zu}$ gänge zu Sprachförderung, Schule und Ausbildung bzw. vorbereitende Angebote (vgl. ebd.). Neben den großen Entfernungen und mangelnden Angeboten führen auch die unterschiedlichen landesgesetzlichen Regelungen des Rechtsanspruchs auf Bildung (Kita- und Schulbesuch) zu großen Unterschieden in der Umsetzung und im Zugang geflüchteter Kinder zu ihren Rechten.

Darüber hinaus ist fraglich, inwiefern Mitarbeiter*innen in den Unterkünften über Angebote frühkindlicher Förderung im Umfeld der Unterkünfte und über die rechtlichen Ansprüche geflüchteter Kinder und ihrer Eltern informiert sind, insbesondere wenn Mitarbeiter*innen nicht frühpädagogisch qualifiziert sind.

Die eingeschränkten Möglichkeiten gesellschaftlicher Teilhabe, von Schutz und Versorgung asylsuchender Kinder, wie ich sie oben beschrieben habe, stellt die Umsetzung der universellen Kinderrechte für alle Kinder in Deutschland in Frage. Trotz nachträglicher vorbehaltloser Ratifizierung der UN-Kinderrechtskonvention im Jahr 2010 unterscheidet die Jugend- und Familienministerkonferenz der Länder (2016) noch immer zwischen Kindergruppen:

„Flüchtlinge mit guter Bleibeperspektive sollen von Anfang an positive Lebensperspektiven in Deutschland erhalten. Vor diesem Hintergrund sehen die Ministerinnen und Minister, Senatorinnen und Senatoren für Familien, Kinder und Jugend der Länder in dem gesetzlichen Auftrag der Kinder- und Jugendhilfe, für ein gelingendes Aufwachsen der jungen Menschen Sorge zu tragen, eine Chance, im Zusammenwirken mit anderen Hilfesystemen einen wichtigen Beitrag zur Unterstützung und zur Integration junger Flüchtlinge und ihrer Familien zu leisten. Alle Maßnahmen und Angebote, die zu einer verbesserten Integration beitragen können, sollen gut zugänglich sein“ (JFMK 2016: 5, Hervorhebung AW).

Während Geflüchtete mit „Bleibeperspektive“ eine Förderung erhalten sollen, werden all jene Kinder, Familien und Jugendliche, die den Kriterien einer „,sicheren Bleibeperspektive“ nicht entsprechen, von einem ,gelingende[n] 
Aufwachsen“ „von Anfang an“ (ebd.) ausgeschlossen. Diese offen diskriminierende Politik widerspricht nicht nur der völkerrechtlichen Konvention der Kinderrechte, in der das Recht eines jeden Kindes auf Nichtdiskriminierung festgeschrieben ist. Der Begriff der Bleibeperspektive koppelt die Teilhabechancen von Kindern und Eltern und die Bedingungen ihres Aufwachsens an einen nicht eindeutig definierten Begriff, der von politischer Willkür zeugt und selbst einem Wandel unterliegt. ${ }^{16}$

Zusammenfassend kann festgestellt werden, dass die Kinderrechte in Deutschland für asylsuchende Kinder nur eingeschränkt gelten, und ausländerrechtliche Vorbehalte, trotz ihrer Rücknahme im Jahr 2010, nach wie vor politisch wirkmächtig sind. ${ }^{17}$

Da die Lebensumstände von Asylbewerber*innen in Deutschland über das Asylgesetz und das Asylbewerberleistungsgesetz (bzw. das SGB XII bei anerkannten Asylantragsteller*innen) geregelt sind, untersuche ich im folgenden Abschnitt, inwiefern die Kinderrechte in diesen gesetzlichen Grundlagen Eingang finden.

\subsection{Kinder im deutschen Asylrecht und -alltag}

Schutzsuchende Minderjährige sowie Alleinerziehende und Familien mit Kindern gelten nach der EU-Aufnahmerichtlinie als besonders schutzbedürftig (Richtlinie 2013/33/EU). Jedes EU-Mitgliedsland ist verpflichtet, die besonderen Bedürfnisse dieser Personen schon bei der Registrierung im Aufnahmeland zu ermitteln und zu beurteilen. So sind Eltern und Kinder möglichst frühzeitig an spezielle Stellen weiterzuleiten, die sie fachlich beraten und die Behörden bei der Festlegung der individuellen Bedarfe unterstützen. Die Schutz-

16 Innerhalb der Gruppe der Kinder wird nicht nur zwischen einheimischen und neu zugewanderten unterschieden. Im asylrechtlichen Diskurs wird weiterhin differenziert zwischen ,guten“, also legitimen Schutzsuchenden und solchen, die diesen Status aus anderen, folglich nicht legitimen Gründen anstrebten und damit kein Recht zu bleiben hätten (vgl. Goodman 2007; Pries 2016). Zu letzterer Gruppe werden auch diejenigen gezählt, die aus sogenannten sicheren Herkunftsländern kommen, einen Antrag stellen und kaum Chancen auf Anerkennung haben. Die Missachtung der Rechte dieser Kinder, wie sie beispielsweise in sogenannten Ausreisezentren von Kindern erfahren werden, findet noch wenig öffentliche Aufmerksamkeit (vgl. Hildegard-Lagrenne Stiftung 2016; für einen Überblick in Großbritannien, Norwegen, Irland und anderen Ländern s. Themenheft Zeitschrift Children \& Society, Nr. 21, 2007, vol. 21, issue 4; Boyden \& Hart 2007: 242).

17 Mit der beabsichtigten Einführung von sogenannten AnkER-Einrichtungen (Aufnahme-, Entscheidungs- und Rückführungseinrichtungen) laut Koalitionsvertrag von CDU/CSU und SPD wird eine Verschlimmerung der Verhältnisse für Kinder und Jugendliche befürchtet (vgl. BKSF-Stellungnahme 17.1.2018). 
bedürftigkeit ist im Rahmen der EU-Richtlinie nicht (bzw. nicht unmittelbar) aus den Fluchtgründen herzuleiten, sondern begründet durch die persönlichen Dispositionen der Antragsteller*innen. In Bezug auf Kinder ist es insbesondere ihr Alter, ${ }^{18}$ das sie im Vergleich zu anderen Asylsuchenden verletzlicher macht (vgl. Schuster 2014).

Aus dieser besonderen Schutzbedürftigkeit von Kindern werden dementsprechend besondere Rechte und Bedürfnisse von Kindern abgeleitet (s. u.). Im Folgenden untersuche ich, wie und ob diese in der asylrechtlichen Praxis Umsetzung finden. Dabei beschränke ich meine Analyse auf die Zeit während des Asylverfahrens, auf die Unterbringung und Versorgung der Kinder sowie ihren Anhörungsprozess und die Möglichkeiten der Familienzusammenführung.

\subsubsection{Unterbringung: Gemeinschaft in Gemeinschaftsunterkünften?}

Die Feststellung der Schutzbedürftigkeit bei Antragstellung auf Asyl sollte sofort dazu führen, dass auch begleitete Kinder kindgerecht untergebracht werden. Nach dem Grundgedanken der EU-Richtlinie (2013/33/EU des Europäischen Parlaments und des Rates der Europäischen Union) ist „ein menschenwürdiges Leben in allen Phasen der Aufnahme zu ermöglichen" (vgl. Schuster 2014: 238 f.). Diese Richtlinie wird ergänzt durch Landesaufnahmegesetze und Aus- und Durchführungsverordnungen. Die Beschreibung von Mindeststandards für die Unterbringung begleiteter geflüchteter Kinder der einzelnen Bundesländer lässt auf eine sehr weite Definition schließen, was jeweils als Ermöglichung eines menschenwürdigen Lebens zu verstehen ist (vgl. Wendel 2014).

Die Situation in den Gemeinschaftsunterkünften stellt sich für Familien mit Kindern als herausfordernd und anstrengend dar.

„Da das gemeinsame Wohnen nicht freiwillig geschieht und zwischen den Betroffenen weder verwandtschaftliche noch von vornherein Freundschaftsbeziehungen bestehen, wird das Leben auf engem Raum in der Regel in Mehrbettzimmern und die gemeinsame Nutzung von Sanitär- und Kücheneinrichtungen sowie - wenn vorhanden - Gemeinschaftsräumlichkeiten als demütigend und belastend empfunden“ (Wendel 2014: 39). (Siehe auch die Beiträge von Scott \& Le, Fichtner \& Trần und Schulz-Algie.)

18 Mit der Bezugskategorie Alter wird ein entsprechender Entwicklungsstand des Kindes angenommen, der rechtlich kodifiziert ist. Davon unabhängig gilt, dass das individuelle Kind über Fähigkeiten, Fertigkeiten und Wissen verfügen kann, die seine rechtlich fixierten Möglichkeiten überschreiten. 
Eltern sind um die Sicherheit ihrer Kinder besorgt, auch weil oftmals eine gemeinsame Sprache fehlt, um sich über Gewohnheiten und Bedürfnisse zu verständigen (vgl. Beauftragte für Flüchtlings-, Asyl- und Zuwanderungsfragen des Landes Schleswig-Holstein zitiert nach Wendel 2014: 39).

\subsubsection{Exkurs Lager (,,camp“) oder Unterkunft (,, site“)?}

Der Begriff Lager, für sich alleinstehend oder in Verbindung mit „Flüchtlingslager", wird in der deutschsprachigen aktuellen Literatur nicht mehr verwendet. Nach eigener Recherche in einer bekannten Suchmaschine wird der Begriff „Flüchtlingslager“ in wissenschaftlichen Abhandlungen und Berichten auf Unterbringungsformen außerhalb Deutschlands bezogen (eigene Recherche Februar 2017). ${ }^{19}$

Mesovic (2014) verweist darauf, dass der Lagerbegriff noch in den 1980er Jahren geläufig war zur Kennzeichnung einer abschreckenden, repressiven Flüchtlingspolitik. Mit der Einführung des Begriffs Gemeinschaftsunterkunft sollte die Assoziation mit dieser Politik entschärft werden (vgl. Mesovic 2014: 7). ${ }^{20}$ Als Gemeinschaftsunterkunft beschreibt das Bundesrecht ein Gebäude, in dem mehrere Personen untergebracht sind und die Räumlichkeiten nicht für andere Zwecke anderweitig zum Wohnen oder Übernachten vermietet werden dürfen (vgl. Wendel 2014: 9). Die gemeinsame Nutzung von Toiletten und Duschen ist charakteristisch für ein „Wohnheim“. Im Zusammenhang mit der Unterbringung - ein Begriff, der aus dem Bereich der Fürsorge für Obdachlose, körperlich und geistig Beeinträchtigte entlehnt ist - begründet die Unterkunft ein Verwaltungsverhältnis zu den sich dort Aufhaltenden. Der Umgang hat „Objektverwaltungscharakter“ (ebd.).

Die Nichtregierungsorganisation Pro Asyl spricht im Zusammenhang mit der Unterbringung von Geflüchteten auch von Bevormundung: Geflüchtete werden zugewiesen, sie können sich weder den Ort der Unterbringung aussuchen noch haben sie ein Mitspracherecht, mit wem sie die Unterkunft teilen. Auch werden eigene Netzwerke, Verwandtschaftsbezüge außerhalb der Kernfamilie bei der Unterbringung nicht berücksichtigt. Mit dem Gesetz zur Einführung beschleunigter Asylverfahren, auch Asylpaket II genannt ist eine Wohnsitzauflage auch für anerkannte Asylbewerber*innen eingeführt worden.

19 Der Begriff Lager wird in Bezug auf den Zweiten Weltkrieg, Vertriebene, Displaced Persons, Kriegsgefangene, Konzentrationslager und später Ost-West-Übergangslager in Deutschland verwendet (vgl. Beer 2014; siehe auch Bochmann 2017 für eine begriffstheoretische Analyse im außereuropäischen Raum).

20 Nach Wendel wurde der Begriff Gemeinschaftsunterkunft historisch zum ersten Mal im Asylverfahrensgesetz von 1982 verwendet. Zur Auswahl standen damals noch andere Begriffe wie Sammelunterkunft oder Sammellager. Mit dem Begriff der Gemeinschaft sind eher positive Konnotationen verbunden, so dass Wendel eine Intention hinter der Begriffswahl vermutet (vgl. Wendel 2014). 
Damit behält der Staat seine regulatorische Funktion und Kontrolle auch über die Anerkennung von Asylbewerber*innen hinaus, ungeachtet realer Arbeitsund Wohnmöglichkeiten am zugewiesenen Ort für anerkannte Asylbewerber*innen.

In Berlin wurden ,,mobile Unterkünfte für Flüchtlinge“ (MUF), auch ,tempohomes" genannt, in Form von Containern aufgestellt. Bereits der Name deutet an, dass es sich hier um eine besondere, provisorische Wohnform für Geflüchtete handelt. Die Lebensdauer von solchen Containern gibt der Hersteller mit drei Jahren an. Die Zuweisung besonderer Wohnformen für ausgewählte Personengruppen widerspricht dem Grundgedanken eines integrativen Miteinanders. Die Entwicklung solcher Containerdörfer entspricht auch nicht der Schaffung bezahlbaren Wohnraums mit langfristiger Perspektive für die wachsende Gruppe derer, die auf bezahlbaren Wohnraum angewiesen sind, unabhängig von ihrem Aufenthaltsstatus als Einheimische oder Zugezogene.

\subsubsection{Versorgung}

Im Rahmen des Asylbewerberleistungsgesetzes ist die medizinische Versorgung von Kindern (und anderen besonders Schutzbedürftigen) auf eine Akutversorgung beschränkt. ${ }^{21}$ In Berlin besteht seit 2009 eine Kooperation mit dem Berliner Netzwerk für besonders Schutzbedürftige. Im Rahmen dieser Kooperation sollen auch die individuellen Bedarfe zum Beispiel geflüchteter Kinder berücksichtigt werden. Das tatsächliche Angebot wird dem (angenommenen) Bedarf an psychotherapeutischer Hilfe für geflüchtete Kinder im Jahr 2016 jedoch nicht gerecht (vgl. Senatsverwaltung 2016). Vor diesem Hintergrund fällt besonders ins Gewicht, dass Träger von Unterkünften für Geflüchtete nicht im Rahmen der Mindeststandards dazu verpflichtet sind, traumatherapeutisch oder traumapädagogisch geschultes Personal, wie es für die traumasensible Beratung und Begleitung von Kindern und Eltern erforderlich wäre, einzustellen. ${ }^{22}$

\subsubsection{Anhörung im Asylverfahren}

Kinder werden in Deutschland erst mit Vollendung ihres 14. Lebensjahres zu ihren Fluchtgründen angehört. Bis zu diesem Alter können Eltern bzw.

21 Alle anderen den Katalog des AsylblG übersteigende Leistungen sind im Vorfeld bei der Leistungsbehörde einzeln zu beantragen, wie beispielsweise Hilfsmittel (Rollstuhl, Brille o. Ä.) oder psychotherapeutische Diagnostiken und Behandlungen ( $\$ 6$ AsylbLG) (Senatsverwaltung 2016) (s. o.).

22 Nach § 6 Abs. 2 AsylbLG sind Dolmetscher*innen und Sprachmittler*innen für Betroffene bereitzustellen. 
rechtliche Vertreter*innen der Minderjährigen eine schriftliche Stellungnahme zu den kindspezifischen Fluchtgründen abgeben (vgl. BAMF DA-Asyl, Familieneinheit $§ 14$ a AsylVfG Stand 01/2016). Die Mitwirkungspflicht von Kindern in der Asylanhörung setzt erst mit diesem Alter ein. Nach der UN-Kinderrechtskonvention ist es das Recht eines jeden Kindes in allen Angelegenheiten, die es betreffen, angehört zu werden (Artikel 12 UNKRK wie auch Artikel 24 EU-Grundrechtecharta). ${ }^{23}$ Die EU-Verfahrensrichtlinie (Artikel 14 EU-Verfahrensrichtlinie 2013/32/EU) schreibt entsprechend eine kindgerechte Durchführung der Asylanhörung vor (vgl. Meysen et al. 2016: 22) ${ }^{24}$ Nach den allgemeinen Bestimmungen des Artikel 5 EU-Richtlinie sind alle Antragsteller*innen über die im Rahmen der Aufnahme gewährten Vorteile sowie über die damit zusammenhängenden Beratungsmöglichkeiten zu informieren. Das muss in einer Sprache erfolgen, „die der Antragsteller versteht oder von der vernünftigerweise angenommen werden darf, dass er sie versteht" (Schuster 2014: 237). Folglich ist über die Information spezifischer Fluchtgründe hinaus, die in der Person des Kindes begründet liegen und die für die Asylantragstellung bedeutsam sein können, auch in einer Sprache bzw. in einer Art und Weise zu beraten, die dem Verständnis des Kindes gerecht wird.

Für die qualifizierte Anhörung von Kindern fehlt es den Behörden jedoch an Personal. Auch sind nicht ausreichend Mitarbeiter*innen zugegen, um mit Eltern und Kindern zu beraten, inwieweit eine Anhörung des Kindes sinnvoll ist (vgl. Bertold 2014). Gemessen an der EU-Verfahrensrichtlinie handeln die zuständigen Behörden nicht angemessen. Das grundsätzliche Recht von Kindern auf Partizipation in dieser für sie zentralen Angelegenheit wird damit in Deutschland nicht zufriedenstellend umgesetzt.

Auch ein weiterer für das Leben von Kindern zentraler Bereich, das $\mathrm{Zu}-$ sammenleben mit ihren Familienangehörigen, soll im Zusammenhang mit der asylrechtlichen Politik und den Rechten von Kindern abschließend kurz betrachtet werden.

23 Eine Anhörung von Kindern zu ihren persönlichen Fluchtgründen ist in Deutschland für die Altersgruppe bis fünf Jahre in der Regel nicht vorgesehen. In Einzelfällen drohender Abschiebung ist „Gelegenheit zur Stellungnahme zu eventuellen eigenen Asylgründen des Kindes einzuräumen“ (BAMF 2016, S. 5). Für Minderjährige gilt mit der Asylantragstellung ihrer Eltern oder eines Elternteils auch ihr Antrag auf Asyl gestellt, auch ohne ausdrücklichen Antrag (Antragsfiktion).

24 In Schweden werden Kinder im Asylverfahren angehört. Die Praxis der individuellen Anhörung von Kindern wird zunehmend auch kritisch gesehen (vgl. Eastmond \& Asher 2011). Seit 2016 sind auch in Schweden asylpolitische Restriktionen in Kraft getreten, die die Situation grundlegend verändert haben (vgl. Parusel 2017). 


\subsubsection{Familienzusammenführung}

Asylbegehrende Geflüchtete, die ihre direkten Familienangehörigen, wie Eltern, minderjährige Kinder und Ehegatten nach Deutschland holen wollen, haben seit der Asylnovelle 2015 keine Möglichkeit mehr dazu, wenn ihnen nur subsidiärer Schutz zuerkannt wurde (s. u.). ${ }^{25}$ Die Koalitionsregierung aus CDU/CSU und SPD führt in ihrer Begründung des Gesetzes zur Aussetzung des Familiennachzugs für subsidiär Schutzberechtigte explizit das Ziel an, darüber die staatliche Steuerung und die Reduzierung vom Zuzug von Flüchtlingen sowie die asylrechtliche Verfahrensbeschleunigung zu erreichen (vgl. BTDrucksache 18/7538: 1). Damit widerspricht die gesetzlich geregelte Aussetzung des Familiennachzugs vom Grundsatz her dem Leitgedanken der vorrangigen Beachtung des Kindeswohls nach Artikel 3 UN-Kinderrechtskonvention, auf das in der Begründung der Gesetzgebung jeweils Bezug genommen werden muss. Es widerspricht auch dem Grundgesetz, das die Familie unter besonderen Schutz stellt (Artikel 6 GG).

Aus kinderrechtlicher Perspektive ist bei jeder Entscheidung, die die Interessen eines Kindes betrifft, die Meinung des Kindes zu berücksichtigen (Artikel 12 UNKRK), so auch der Kommentar des UN-Kinderrechteausschuss (vgl. WD 2016: 6, Fußnote 10). Das Kindeswohl (Artikel 3 UNKRK) ist in jedem Fall vorrangig bei behördlichen Entscheidungsprozessen zu berücksichtigen und als wesentliche Leitlinie der nationalen Gesetzgebung zu verstehen, die es bestmöglich nicht nur umzusetzen, sondern auch stets in der Realisierung zu verbessern gilt (vgl. WD 2016: 6). Darüber hinaus ist der Familiennachzug über EU-Recht bzw. nationales Recht in einem komplexen System geregelt und von zahlreichen Kriterien abhängig ( $§ 27 \mathrm{f}$. AufenthaltsG). Die Entscheidung liegt im Ermessen der Behörden, die die Anträge ,unvoreingenommen prüfen und nach objektiver Sachlage entscheiden" müssen (vgl. Wissenschaftlicher Dienst 2016: 5).

25 Seit dem 1.8.2018 dürfen im Rahmen der Neuregelung zum Familiennachzug der subsidiär Schutzberechtigten maximal 1000 Angehörige pro Monat zu ihren engsten Angehörigen nachziehen. 


\section{Kampf um die diskursive Hegemonie - geflüchtete Kinder im Spannungsfeld von Kinderrechten und Asylpolitik}

Die Betrachtung ausgewählter Lebensbereiche asylbegehrender Kinder und ihrer Familien in Deutschland hat gravierende Rechtsverletzungen, Schwachstellen und Widersprüche in der aktuellen Asylpolitik aufgezeigt.

Mit Bezug auf die von Laclau und Mouffe verfasste Theorie hegemonialer Diskurse (Laclau \& Mouffe 1985) gehe ich nachfolgend der Frage nach, wie derartige Widersprüchlichkeiten und Rechtsverletzungen zu verstehen sind. ${ }^{26}$ Hierbei interessiert insbesondere die Frage, wie „politische Identitäten“ (vgl. Vitus \& Lidén 2010) bzw. Identitäten von Subjekten (vgl. Reckwitz 2006) an den Bruchstellen konfligierender Diskurse (neu) hergestellt werden. Welche „Identität" wird asylsuchenden Kindern im Wettstreit zwischen Kinderrechtsdiskurs und Asylrechtsdiskurs zugeschrieben? Welchen Stellenwert nimmt die UN-Kinderrechtskonvention im migrationspolitischen und asylrechtlichen Diskurs ein? Entlang dieser Fragen wird im Folgenden die Figur des asylbegehrenden Kindes diskutiert.

Nach Laclau wird die Auseinandersetzung um die Fundierung der Gesellschaftsordnung als ein offener und unabgeschlossener Konflikt geführt, in deren Zuge sich neue soziale und politische Einflusssphären entwickeln. Anders als bei Marx sei der Kampf um Hegemonie nicht nur durch die objektiven ökonomischen Verhältnisse und die Klassenpositionen bestimmt, sondern eher als kontingent, mit offenem Ende, zwischen konkurrierenden Diskursen und Antagonismen zu verstehen (vgl. Vitus \& Lidén 2010: 63). Der diskursive Kampf um die Bestimmung der Identitäten von Subjekten sei Ausdruck des Ringens um die gemeinsame Ordnung der sozialen Verhältnisse. Gemeint ist die hegemoniale Auseinandersetzung über die Grundlagen einer gemeinsamen Identität und zentraler gesellschaftlicher Orientierungen (vgl. Schäfer 2016). Hiernach produzieren Diskurse eine spezifische Sinnwelt, eine „Ordnung der

26 Die poststrukturalistische Kulturtheorie von Laclau basiert auf der Marxismusanalyse Antonio Gramscis und seiner Theorie der Hegemonie und dem Dekonstruktivismus von Jacques Derrida (vgl. Reckwitz 2006: 340). Laclau hebt die kategoriale Unterscheidung von Diskurs und Praxis auf. Über den gesellschaftlichen Diskurs wird die Identität von Subjektpositionen festgelegt. In dem Versuch, hegemoniale Projekte wie den Neoliberalismus gesellschaftlich durchzusetzen, wird regelmäßig versucht, nur eine bestimme Ordnung gesellschaftlicher Verhältnisse als möglich zu präsentieren, zentral ist hierbei die Identität von Subjektpositionen (Reckwitz 2006: 343). Aus der Perspektive der Kritischen Kulturtheorie Laclaus ist das, was die moderne Sozialtheorie das „Soziale“ oder die „Gesellschaft“ nennt, ,nichts anderes als eine Agglomeration von Diskursen“ (ebd.: $341)$. 
Dinge", in der den Dingen erst eine bestimmte Bedeutung zugeschrieben und ein bestimmtes Handeln möglich wird. ${ }^{27}$ Im Zentrum konkurrierender Diskurse stehen demnach jeweils unterschiedliche Fragen der politischen Identität. Diese „Identitätsdiskurse“ ringen um Hegemonie. Die eigene Perspektive beansprucht eine hegemoniale Positionierung, also gesellschaftliche Definitionsmacht gegenüber anderen Perspektiven und den sich daraus eröffnenden praktischen Möglichkeiten (vgl. Schäfer 2016: 94). Nach Laclau und Mouffe ist es das Verhältnis von Universalität und Besonderheit bzw. Partikularismus, das in und zwischen diesen Identitätsdiskursen verhandelt wird (vgl. Laclau \& Mouffe 1985). Es geht also m.E. darum, welche Werte und ,identities“ (vgl. Vitus \& Lidén 2010: 64) transzendieren, also losgelöst von Zeit und Raum Gültigkeit beanspruchen können, gegenüber jenen, die an spezifische Verhältnisse, an Ort und Zeit gebunden und damit als separat zu betrachten und den allgemeingültigen, „universellen“ Werten als unterlegen verhandelt werden. Da hegemoniale Diskurse Allgemeingültigkeit und Alternativlosigkeit beanspruchen, projizieren sie das radikal Andere nach außen und markieren es als nicht dazugehörig. Gleichwohl sind sie existentiell auf das Andere, das Nichtdazugehörige, das Partikulare angewiesen, um sich von diesem abgrenzen zu können (vgl. Reckwitz 2006: 345; Laclau 1996). Darin begründe sich die unauflösbare gegenseitige Bedingtheit antagonistischer Diskurse.

In Bezug auf die Figur des asylsuchenden Kindes beschreiben Vitus und Lidén einen „struggle“ der Diskurse mit folgenden antagonistischen Positionen:

„The category [asylum seeking child] on the one hand refers to the political identity of , asylum-seeker' created through antagonistic struggles about inclusion in or exclusion from the nation state, i. e. questions of nationality and national citizenship. On the other hand, the category , children ' refers to the political identity of ,the child' created through discursive struggles about children's vulnerability, followed by the claim for childrens' rights “ (Vitus \& Lidén 2010: $65) \cdot{ }^{28}$

Die Figur ,asylum-seeking child“ (vgl. Vitus \& Lidén 2010) ist demnach Produkt und Ausdruck anhaltender politischer, sozialer und historisch kontextbezogener diskursiver Aushandlungsprozesse zwischen der politischen Identität „Kind“ und der politischen Identität „Asylbewerber*in“, ohne dass eine der politischen Zuschreibungen einen Ausschließlichkeitsanspruch erheben könn-

27 Laclau spricht von der ,strukturellen Totalität“" von Diskursen als einem spezifischen System von Unterscheidungen (vgl. Reckwitz 2006: 341).

28 Die Kategorie asylbegehrendes Kind verweist einerseits auf die politische Figur der Asylbewerber*innen. Mit ihr verknüpft sind die widerstreitenden politischen Auseinandersetzungen um Fragen von (nationalstaatlich begründeter) Zugehörigkeit bzw. Ausgrenzung. Auf der anderen Seite wird über die Kategorie Kind auf die diskursiv geführten Auseinandersetzungen um die Vulnerabilität von Kindern und die daran anschließenden eingeforderten Kinderrechte Bezug genommen. 
te; vielmehr ist die Figur hochambivalent. In ihrer politischen Zuschreibung als ,asylsuchendes Kind“ wird eine dritte „Identität“ kreiert, die nicht in den definierten politischen Identitäten von „Flüchtling“ oder „Kind“ aufgehen kann.

Nach dieser knappen theoriegeleiteten Skizze diskutiere ich nun diskursive Positionierungen asylbegehrender Kinder anhand empirischer Studien aus Schweden, Norwegen, dem Vereinigten Königreich und Dänemark und weite damit den Rahmen auf die in Europa geführten Diskurse zu Kinderrechten und „Flüchtlingen“" aus.

Es gibt nicht „den“ europäischen „Flüchtlingsdiskurs“. Vielmehr werden Geflüchtete diskursiv unterschiedlich verhandelt (so z. B. als Opfer von Krieg und Gewalt, als Gefährdete mit besonderem Schutzanspruch, als Gefährdung für die aufnehmende Gesellschaft). Gemeinsam ist diesen Diskursen, dass das Individuum in der „Masse der Flüchtlinge“ untergeht, keine Biografie und keine individuellen Charakterzüge hat (vgl. Emcke 2016; Holmes \& Castañeda 2016). Im politischen und medialen Diskurs werden seit den 1990er Jahren Asylbewerber*innen als Gruppe oder „Strom“ und „Masse“ verhandelt und allenfalls auf „objektivierte juristische Fälle“ reduziert (vgl. Vitus \& Lidén 2010).

Demgegenüber haben schutzsuchende geflüchtete Kinder einen entsprechenden Anspruch als Subjekte und individuelle Rechtsträger*innen über die UN-Kinderrechtskonvention. Wer angesichts der asylpolitischen Gemengelage jedoch politisch unter die Kategorie Kind fällt - mit entsprechend garantierten Rechten - ist vor diesem Hintergrund, auch für Deutschland (s. u.) nicht so eindeutig, wie der Titel der Publikation von Unicef „In erster Linie Kinder“ nahelegen möchte (vgl. Berthold 2014).

Für Dänemark und Norwegen untersuchen Lidén und Vitus die Frage, wie politische Identitäten von asylbegehrenden Kindern an den Bruchstellen antagonistischer Diskurse konstruiert werden (vgl. Vitus \& Lidén 2010). Obwohl beide Länder die UNKRK ratifiziert haben, werden asylsuchende Kindern unterschiedliche „Identitäten“ diskursiv zugewiesen.

Für Dänemark stellen die Autorinnen fest, dass der ausländerrechtliche Diskurs gegenüber den Kinderrechten für asylsuchende Kinder dominiert. Ähnlich wie in Deutschland wurde in Dänemark die UNKRK ratifiziert, jedoch nicht in die nationale soziale oder asylrechtliche Gesetzgebung eingegliedert, mit weitreichenden Folgen für asylbegehrende Kinder: Asylsuchende Kinder können zwar die Schule besuchen, haben aber anders als dänische Kinder keinen Anspruch auf Schulkredit. Im Asylprozess werden sie nicht individuell angehört und haben keine Möglichkeit, individuell aus humanitären Gründen einen Aufenthalt zu beantragen (vgl. Vitus \& Lidén 2010: 78). Asylbegehrende Kinder werden als Rechtsträger*innen in Verbindung mit der Einheit der Familie und damit immer im Zusammenhang mit ihrer Familie in der Ausländergesetzgebung (Danish Alien Act) gesehen. 
Anders in Norwegen: Das Land versteht sich als Vorreiter für die Belange von Kindern und in der Umsetzung von Partizipationsrechten von Kindern. Im Jahr 2003 wurde die Kinderrechtskonvention im norwegischen Menschenrechtsgesetz (Norwegian Human Rights Act) implementiert. Seither müssen sämtliche Gesetze angepasst werden an die vom UN-Kinderrechtsausschuss herausgestellten fundamentalen Prinzipien (,framework") und konkrete Artikel der UNKRK. Dies hat nicht nur zu Gesetzesänderungen geführt. Insbesondere die Frage nach den „best interests of the child“, das Recht auf Beteiligung von Kindern und die Rechte von asylbegehrenden Kindern führte zu öffentlichen Debatten, bis hin zur Berücksichtigung kindbezogener Verfolgung als Asylgrund. Berücksichtigt werden muss auch die Qualität der Bindungen des asylbegehrenden Kindes zum Land Norwegen, so dass aufgrund der emotionalen Bindung des Kindes an das Aufnahmeland auch seine Familie ein Aufenthaltsrecht erhalten kann. Entsprechend des Prinzips, bei knappen Ressourcen denjenigen zu helfen, die am meisten darauf angewiesen sind, können eher Kinder aus humanitären Gründen ein Aufenthaltsrecht zugesprochen bekommen als Erwachsene. Nach Vitus und Lidén setzt Norwegen die Kinderrechte für asylbegehrende Kinder um: Die Kinder besuchen die reguläre Schule, ihre Rechte werden im Asylanhörungsprozess gewahrt und wenn das Kriterium der entwickelten Bindung an die norwegische Gesellschaft (nach dreijährigem Aufenthalt) hinzugezogen wird, haben sie Aussicht auf einen Aufenthaltstitel (vgl. Vitus \& Lidén 2010).

Allerdings können konträr zu dieser kinderrechtsbezogenen Asylrechtslage die kindbezogenen Aufenthaltsgründe komplett entfallen, wenn Eltern bei der Einreise oder Asylantragstellung falsche Angaben machen. Das Kind wird dann trotz eigener Aufenthaltsberechtigung gemeinsam mit den Eltern abgeschoben. Das kinderrechtlich bestimmte Asylrecht wiegt demzufolge weniger als das allgemeine Asylrecht in Norwegen. In der Begrifflichkeit von Partikularem und Universellem nach Laclau stellt das im Kind begründete Aufenthaltsrecht die besondere Situation, das Partikulare dar, das dem universellen, dem allgemeinen Asyl- und Migrationsrecht unterliegt. Damit entsteht in diesem Fall die Situation, dass Kindern weder die Rechte der UN-Kinderrechtskonvention zuteilwerden noch eigenständige Asylrechte (vgl. Vitus \& Lidén 2010: 78). Unter derartigen Bedingungen entsteht eine neue politische „Identität": die Kategorie der ,anderen Kinder" mit unklarem bzw. widersprüchlichem Rechtsbezug.

„While Norway has the political intentions of taking responsibility for treating asylum-seeking children as ,children' in the terms defined in the CRC, both practical difficulties and political priorities make living up to these intentions difficult" (Vitus \& Lidén 2010: 78).

In Schweden ist den Behörden seit 2006 Interpretationsspielraum zwischen Rechtslage und Rechtspraxis eingeräumt worden in der Umsetzung der 
Kinderrechte mit widersprüchlichen Folgen für begleitete asylsuchende Kinder (Eastmond \& Asher 2011: 1188). Entgegen der im Vorfeld der Einführung des neuen Ausländerrechts geführten Debatten ${ }^{29}$ beziehen sich die schwedischen Ausländerbehörden in 71 Prozent der Fälle auf das Kindeswohl in ihrer Entscheidung den Asylantrag abzulehnen (demgegenüber wird nur in 17 Prozent der positiven Aufenthaltsentscheidungen mit dem Kindeswohl argumentiert). Das Prinzip das Kindeswohl in allen Entscheidungen zu berücksichtigen wird nach Lundberg angesichts dieser Zahlen wohl eher zur Legitimierung von Ablehnungen genutzt. (vgl. Eastmond \& Asher 2011: 1195). ${ }^{30}$

\begin{abstract}
„While the principle of ,the best interest of the child" has been given an increasingly prominent place in Swedish Aliens Law and Migration Board guidelines, in practice children's voices and their own individual grounds are rarely given corresponding weight, even though, as a semantically flexible concept, ,the child's best interest' would, in principle allow consideration of a broad range of different and individual situations. At the structural level, these discrepancies reflect the position of asylum-seeking children at the intersection between two opposing principles 'the nation state's interest in controlling its borders and the universalized rights of children as enshrined in the CRC. Thus the Swedish Aliens Act placed greater emphasis on children's best interest but also made clear that children's interests remain subordinated to the state's need to regulate immigration" (Eastmond \& Ascher 2011: 1195) ${ }^{31}$.
\end{abstract}

Für Großbritannien beschreibt Giner (2007), dass auf asylsuchende Kinder in zwei unterschiedlichen rechtlichen Rahmen Bezug genommen wird: Demnach werden Asylbewerber*innen vorrangig im Kontext der Sicherung nationaler Grenzen in Großbritannien verhandelt. Ihnen begegnet institutionalisiertes Misstrauen, ihr Zugang zu wohlfahrtsstaatlichen Angeboten wird eingeschränkt und in Einzelfällen werden sie sogar verhaftet. Im Gegensatz dazu wird die ,politische Identität" von Kindern als ,,inclusive childhood“ verstanden und der Diskurs auf der Grundlage der Kinderrechte mit Schutzanspruch für alle Kinder geführt (vgl. Giner 2007: 249). Nach Vitus und Lidén werden damit Aspekte der Sorge (,care") und Entwicklung von Kindern angesprochen (vgl. Vitus \& Lidén 2010: 63), Konzepte, die dem europäischen Kindheitsbild der Moderne entlehnt sind (vgl. Wihstutz 2018).

29 Im Vorfeld der Einführung des neuen Ausländerrechts im Jahr 2006 wurde argumentiert, dass die Prüfung des Asylgesuchs von Kindern gegenüber einem Antrag von Erwachsenen wohlwollender auszufallen habe, um ,the best interests of the child“ vorrangig zu berücksichtigen (vgl. Eastmond \& Asher 2011: 1194).

30 Bis 2015 galt die Asylpolitik Schwedens als relativ „großzügig“: Gemessen an seiner Bevölkerungsgröße nahm Schweden mehr Asylbewerber*innen auf als andere EUStaaten. Seit 2016 sind auch in Schweden asylpolitische Restriktionen in Kraft getreten, die die Situation grundlegend verändert haben (vgl. Parusel 2017).

31 Vgl. auch Liebel 2018 zur Kritik an der paternalistischen Interpretation des Prinzips des Kindeswohls der UN-Kinderrechtskonvention. 
Die Auslegungen ausländerrechtlich und kinderrechtlich relevanter nationaler Gesetze durch die Behörden sind auch in Deutschland für begleitete asylantragstellende Kinder nicht eindeutig. Das behördliche Handeln hat sich sowohl am Aufenthaltsgesetz (neuer $\S 104$ Abs. 13 AufenthG) als auch an der Maßgabe der UN-Kinderrechtskonvention zu orientieren. ${ }^{32}$ Dabei verfolgen beide im Kern unterschiedliche Ziele (vgl. WD 2016: 10). Bei der Novellierung des Aufenthaltsgesetzes hält sich der Gesetzgeber nicht an seine mit der Ratifizierung der UN-Kinderrechtskonvention eingegangene Verpflichtung, neue Gesetze in Bezug auf das Kindeswohl nach Artikel 3 UNKRK zu begründen. Im Zusammenhang mit der Aussetzung des Familiennachzugs fällt auch auf, dass auf den Widerspruch zum Grundgesetz nicht eingegangen wird und damit der Verstoß billigend in Kauf genommen wird (vgl. WD 2016: 5, Fußnote 6). Neben dem Grundgesetz, das die Familie unter den besonderen Schutz der staatlichen Ordnung stellt (s. o.), steht die Regelung zur Aussetzung des Familiennachzugs auch im Widerspruch zu Artikel 8 der Europäischen Menschenrechtskonvention, dem Recht auf Familiennachzug im Rahmen des Rechts auf Achtung des Familienlebens und Artikel 24 Abs. 3 der EU-Grundrechtecharta (,Jedes Kind hat Anspruch auf direkte Kontakte zu beiden Elternteilen"). Die Aussetzung des Familiennachzugs für Personen mit subsidiärer Schutzanerkennung widerspricht darüber hinaus grundsätzlich dem Prinzip der UN-Kinderrechtskonvention des ,best interests of the child“ (vgl. Committee on the Rights of the Child 2013).

Aus einschlägigen Studien ist bekannt, dass die Familie für die Gesundheit und für die Bewältigung von Flucht und des fremden Alltags in der Aufnahmegesellschaft eine sehr hohe Bedeutung für Kinder und Eltern hat (vgl. Fegert et al. 2017; Maragel \& Manachi 2018). Aufgrund ihrer besonderen Vulnerabilität und Abhängigkeit haben geflüchtete Kinder ein Recht, mit ihren Eltern vereint zu werden (Artikel 10 UNKRK). ${ }^{33}$ Die Rechte von Erwachsenen wiegen jedoch ungleich schwerer als die eines Kindes, beispielsweise in Bezug auf das Aufenthaltsrecht: Wie am Beispiel Schweden erläutert wurde, wird der Aufenthaltstitel des Kindes den Rechten der Eltern untergeordnet. Die Familie wird gemeinsam abgeschoben, auch wenn das Kind einen eigenständigen Aufenthaltstitel erworben hat (vgl. Vitus \& Lidén 2010). Für die USA beschreibt Bhabha (2006) eine ähnliche Problematik: Der Aufenthaltstitel der Kinder als

32 In Deutschland wenden Verwaltungen und Gerichte entsprechende Rechte unterschiedlich an (vgl. WD 2016: 9). Auf die unterschiedliche Anerkennungs- und Abschiebepraxis zwischen den Bundesländern weist z. B. die Studie von Riedel und Schneider (2017) hin. Die Autor*innen weisen nach, dass das Asylrecht in Deutschland nicht einheitlich in den Bundesländern umgesetzt wird. Nach Riedel und Schneider unterscheiden sich die Anerkennungsquoten so systematisch, dass diese Praxis als gravierende Ungleichbehandlung zu benennen ist (vgl. Riedel \& Schneider 2017).

33 Der Europäische Gerichtshof hat jüngst in einem Urteil das Recht von minderjährigen Geflüchteten bekräftigt, ihre Eltern im Rahmen vom Familienzusammenführung nachzuholen (Zeit online 12.4.2018). 
„citizens“ und die Mitbestimmungsrechte von Kindern sowie ihr Recht, mit der Familie vereint zusammenzuleben, werden in dem Moment hinfällig, in dem Eltern abgeschoben werden. Als Minderjährige haben sie nicht das Recht, für sich bzw. für ihre Familien zu sorgen.

„Family unity and familiy reunification are seen as adult entitlements, perks, rewards for good parenting or for responsible compliance with immigration laws. They are not treated as children's rights []. It [migration policy] considers children possessions of adults. Children's citizenship is in effect inchoate“ (Bhabha 2006: 1530).

Wie widersprüchlich die Rechte von Kindern auch in Deutschland gesellschaftlich diskutiert werden, zeigt eine repräsentative Forsa-Umfrage im Auftrag des Deutschen Kinderhilfswerks (spiegel online 2017). Mehr als die Hälfte der Deutschen (52 Prozent) stimmt der Aussage zu, dass Flüchtlingskinder nicht von Anfang an die gleichen Möglichkeiten wie deutsche Kinder haben sollten. Demgegenüber sind 42 Prozent der Befragten dafür, dass geflüchtete Kinder die gleichen Zugänge zu Bildung erhalten sollten. Der Umfrage zufolge hat sich binnen eines Jahres die überwiegend positive Einstellung gegenüber den Bedürfnissen von Flüchtlingskindern verschlechtert. Nur bei den unter 30Jährigen stimmt eine Mehrheit (58 Prozent) für eine Gleichbehandlung der Kinder (vgl. ebd.). ${ }^{34}$ Das heißt, der Anspruch „,universeller Gültigkeit“ der Kinderrechte für alle Kinder in diesem Land ist aktuell kein allgemeingültiger Grundkonsens in der deutschen Gesellschaft. Vielmehr verweisen die Umfragewerte auf konkurrierende Überzeugungen bezüglich der Rechte und Möglichkeiten geflüchteter Kinder.

Die Behörden stehen vor dem Dilemma, bei geltender Rechtslage zwischen (aufenthalts-)gesetzeskonformen und (UN-Kinderrechts-)Konventionskonformen Entscheidungen treffen zu müssen. Lewek und Naber (2016) ziehen in ihrem „Lagebericht zur Situation der Flüchtlingskinder in Deutschland“ das Fazit, dass die Versorgung und Begleitung von Kindern mit und ohne Familienangehörige oftmals zufällig erfolge. Ausländerrechtliche Kriterien wie das Herkunftsland und die damit vermutete Bleibeperspektive scheinen in vielen Fällen dem Kindeswohl übergeordnet zu werden (vgl. Lewek \& Naber 2016: 2).

Die Selbstbeschreibung als kinderfreundliches Land, das sich für die Kinderrechte und den Zugang von Kindern zu wohlfahrtsstaatlichen Angeboten

34 Nach Parteien differenziert ist eine Mehrheit von Unions- und FDP-Anhänger*innen (52 und 59 Prozent) für ungleiche Behandlung, bei der SPD 41 Prozent und bei der Linken 40 Prozent, bei den Grünen 25 Prozent. 93 Prozent der AfD Anhänger*innen sind für die Ungleichbehandlung, 7 Prozent hatten keine Meinung dazu. Bei der Frage des Familiennachzugs sprachen sich 42 Prozent der Befragten dafür aus, dass Eltern und minderjährige Geschwister der Kinder nach Deutschland kommen sollen dürfen (vgl. www.spiegel.de 20.6.2017; Pressemitteilung DKHW 19.6.2017). 
wie zum Beispiel Bildung und Gesundheit einsetzt, hindert Regierungen nicht, Maßnahmen zu erlassen, die die Rechte und Interessen von Kindern in der unbestimmten Grauzone zwischen Asyl- und Kinderrechten verletzen. Vielmehr trägt die auf Abschreckung und Schließung der Grenzen ausgerichtete Asylund Migrationspolitik nationalstaatlicher Regierungen dazu bei, die Rechte und Lebensbedingungen schutzsuchender Kinder und ihrer Familien im Aufnahmeland akut zu verschlechtern (vgl. Boyden \& Hart 2007; Zeit online 2017).

\section{Zusammenfassung und Ausblick}

Die exemplarische Skizze asylrechtlicher Diskurse in Deutschland, Dänemark, Norwegen, Schweden und Großbritannien stellt heraus, dass schutz- und asylsuchende Kinder vorrangig als Asylbewerber*innen verhandelt und im Zweifelsfall ihre verbrieften Rechte als „Kinder“ den Interessen nationalstaatlicher Migrationspolitik untergeordnet werden (vgl. Boyden \& Hart 2007; Eastmond \& Asher 2011; Giner 2007; Goodman 2007). Dies ist selbst in Norwegen der Fall, einem Land, das als Vorreiter in der Umsetzung kinderrechtlicher Gesetze mit Verfassungsrang gilt (vgl. Vitus \& Lidén 2010; Lidén \& Rust 2007).

Laclau und Mouffe (1985) definieren den diskursiven Streit als notwendigen Ausdruck einer radikal verstandenen Demokratie (vgl. Nonhoff 2007), in der um die hegemoniale Position gerungen werde und gerungen werden müsse. Die hegemoniale Durchsetzung universeller Werte gegenüber den partikularen geht zurück auf diskursive Auseinandersetzungen, Verhandlungen und Anfechtungen in allen Bereichen der Gesellschaft, so auch in Politik, Medien, Wissenschaft und Zivilgesellschaft. In einer ,radikal" verstandenen Demokratie gibt es demnach keinen ,neutralen“ Standpunkt, der im „Außen“ die Richtigkeit oder Falschheit des Arguments beurteilen könnte. Vielmehr seien praktische Verstrickungen in der Auseinandersetzung zu benennen und anzuerkennen (vgl. Schäfer 2016: 97).

In Bezug auf die politische Figur asylsuchender Kinder in Deutschland ist die fehlende Verankerung der Kinderrechte im Grundgesetz, wie oben dargelegt, folgenreich. Doch auch ein rechtlich verankerter Vorrang des Kindeswohls/-interesses wie in Schweden und Norwegen garantiert gegenüber asylsuchenden Kindern nicht die tatsächliche Berücksichtigung des Kinderrechtsprinzips, die Berücksichtigung ihrer Interessen (Kindeswohl, Artikel 3 UNKRK) in der Praxis. Wie meine Ausführungen zum Verhältnis von Asylrecht und Kinderrechten gezeigt haben, dominieren nationalstaatlich orientierte Interessen und Werte im Diskurs auch hier. Vor diesem Hintergrund ist die Frage nach dem Stellenwert der Kinderrechte in den gegebenen aktuellen 
gesellschaftlichen Verhältnissen zu stellen. Von welchem Kinderbild wird ausgegangen? Was bedeuten Partizipation, Schutz und Versorgung, wenn diese wie oben diskutiert nicht für schutzsuchende Kinder gewährleistet werden?

Die Erfahrungen von Kindern mit Gewalt und Krieg und der erzwungenen Flucht weltweit passen nicht in das Ideal von Kindheit, das, als Phase der Sicherheit und Entwicklung, frei von der Last der Verantwortung imaginiert wurde und lange Zeit insbesondere im europäisch-(nord-)amerikanischen Denken verankert war. Die Fluchterfahrungen konstituieren regelrecht eine Antithese zu diesem Kindheitsideal (vgl. Boyden \& Hart 2007: 246). Wie ich in diesem Beitrag dargestellt habe, sind es auch die spezifischen Verhältnisse und Prozesse im aufnehmenden Land, die die Vulnerabilität asylsuchender Kinder mit ihren Familien nicht nur bedingen, sondern sogar verschärfen können. ${ }^{35}$ Die aktive Beteiligung von Kindern, ihre Interessensäußerungen und ihre soziale Eingebundenheit sind stärker als bisher auch rechtlich zu berücksichtigen. ${ }^{36}$ Wie ich am Beispiel der Diskussion um die Aufnahme von Kinderrechten ins Grundgesetz angedeutet habe, ist ein solches Verständnis von (Kinder-)Rechten durchaus auch als ambivalent zu verstehen und nicht widerspruchsfrei, was den Schutz, die Beteiligung und Versorgungsrechte von Kindern anbelangt (vgl. Bhabha 2006).

Im Diskurs um die Rechte geflüchteter Kinder zeigt sich, wie sich eine Gesellschaft selbst definiert.

„Wie Deutschland mit den Herausforderungen der Flüchtlingswanderung des Jahres 2015 umgeht, entscheidet nicht nur über die unmittelbare Zukunft der direkt betroffenen Menschen, sondern auch über das längerfristige Selbstverständnis der Gesellschaft" (Pries 2016: 8).

Mit der Skizzierung des Spannungsverhältnisses zwischen Kinderrechten und Asylrecht und -politik wurde der Versuch unternommen, die Implikationen sowie Widersprüchlichkeiten der Rechte asylbegehrender Kinder aufzuzeigen. Damit verknüpft ist der Anspruch, auf die aus kinderrechtlicher und damit aus

35 Damit soll nicht das Leid und der Schrecken der Erfahrungen von Kindern vor und während der Flucht verharmlost werden oder ihre Schutzbedürftigkeit in Zweifel gezogen werden. Unter den gegebenen bestimmten Bedingungen ist es für Kinder und Eltern unter Umständen erforderlich, sich als Opfer zu präsentieren, um Zugang zu ansonsten verwehrten Ressourcen zu erlangen (vgl. Boyden \& Hart 2007: 246 Bühler-Niederberger 2011; Beitrag von Fichtner \& Trần).

36 Ihre aktive Rolle und soziale Einbettung gilt es stärker kontextbezogen zu reflektieren, wie während des Asylprozesses und bei der Bewältigung im Alltag im Aufnahmeland (vgl. White; für einen Überblick der Diskussion Boyden \& Hart 2007: 246). So lautet eine Kritik an der UN-Kinderrechtskonvention, dass sie am individualistischen Kindheitsbild (der westlichen Moderne) orientiert ist und den Kontext gelebter Kindheiten nicht ausreichend berücksichtigt, wie beispielsweise die Interessen der Kinder als soziale Akteur*innen mit spezifischen Aufgaben und Verpflichtungen im Rahmen von Familie und Gemeinschaft (vgl. Wihstutz 2017; Liebel et al. 2008: 431). 
menschenrechtlicher Perspektive unzureichend gesicherten Lebensverhältnisse begleiteter junger asylsuchender Kinder aufmerksam zu machen und schließlich auf normative Handlungsorientierungen (vgl. Pries 2016) von Politik und Gesellschaft einzuwirken (siehe auch den Beitrag von Wihstutz „Zentrale Erkenntnisse und Empfehlungen“).

\section{Literatur}

BAJ-Bundesarbeitsgemeinschaft Kinder- und Jugendschutz (2016): Geflüchtete Kinder und Jugendliche. Dossier 1/2016, https://www.bag-jugendschutz.de/ PDF/Dossier-gefluechtete_Kinder.pdf (Zugriff 15.2.2017).

Beer, Mathias (2014): Die deutsche Nachkriegszeit als Lagergeschichte - Zur Funktion von Flüchtlingslagern im Prozess der Eingliederung. In: Bispinick, Henrik \& Hochmuth, Katharina (Hrsg.) Flüchtlingslager im Nachkriegsdeutschland. Migration, Politik, Erinnerung. Berlin: Ch.Links Verlag, S. 47- 72.

BKFS-Stellungnahme (2018): BKFS-Stellungnahme zu den Sondierungsergebnissen von SPD und CDU/CSU für eine Regierungskoalition. Bundeskoordinierung Spezialisierter Fachberatung gegen sexualisierte Gewalt in Kindheit und Jugend (BKFS), http://www.bundeskoordinierung.de/de/article/84.koalitionsverhandlungen.html, (Zugriff 18.1.2018).

Bochmann, Annett (2017): Soziale Institution Lager: Theoretische Grundlagen, Flüchtlingslager und die Macht der lokalen Mikrostrukturen. In: Lessenich, Stephan (Hrsg.): Geschlossene Gesellschaften. Verhandlungen des 38. Kongresses der Deutschen Gesellschaft für Soziologie in Bamberg 2016, http://publikationen.soziologie.de/index.php/kongressband_2016/article/view/480 (Zugriff 17.8.2018).

Boyden, Jo \& Hart, Jason (2007): The Statelessness of the World's children. In: Children \& Society 21,Nr. 4 (2007), S. 237-248.

BT-Drucksache 18/7538 (2017): Gesetzentwurf der Fraktionen der CDU/CSU und SPD. Entwurf eines Gesetzes zur Einführung beschleunigter Asylverfahren, http://dip21.bundestag.de/dip21/btd/18/075/1807538.pdf (Zugriff 13.10.2017).

Bundesamt für Migration und Flüchtlinge (BAMF) (2017): DA-Asyl. 25.4.2017. Familieneinheit., https://www.proasyl.de/wp-content/uploads/2015/12/DAAsyl-April-2017.pdf (Zugriff 14.1.2019)

Bundesfachverband unbegleitete minderjährige Flüchtlinge e. V. (BumF) (2017): Zur Reform des Gemeinsamen Europäischen Asylsystems (GEAS), https:// www.nds-fluerat.org/wp-content/uploads/2017/10/GEAS_BumF_Stellungnahme.pdf (Zugriff 11.6.2018).

Bundesfachverband unbegleiteter minderjährige Flüchtlinge e. V. (BumF) (2016): Fact-finding zur Situation von Kindern und Jugendlichen in Erstaufnahmeeinrichtungen und Notunterkünften. Zusammenfassender Bericht November 2015Januar 2016. Mirjam Lewek und Tobias Klaus für den BumF e. V., beauftragt 
vom Deutschen Komitee für UNICEF e. V., https://www.b-umf.de/images/ UNICEF_BUMF_FactFinding_Fl\%C3\%BCchtlingskinder.pdf (Zugriff 19.6. 2018).

Bundesverfassungsgericht (BVerfG): BVerfG, 29.07.1968, Aktenzeichen $1 \mathrm{BvL}$ 20/63; 1 BvL 31/66; 1 BvL 5/67; Leitsätze. https://opinioiuris.de/entscheidung/1563 (Zugriff am 14.1.2019).

Bundesweite Arbeitsgemeinschaft der psychosozialen Zentren für Flüchtlinge und Folteropfer (BAfF): Leitfaden zur Beantragung einer Psychotherapie für Geflüchtete, www.baff-zentren.org/wp-content/uploads/2017/03/BAfF_Arbeits hilfe_Therapiebeantragung.pdf (Zugriff 12.10.2017).

Bundeszentrale für Politische Bildung (bpb) (2013): Vor zwanzig Jahren: Einschränkung des Asylrechts 1993, http://www.bpb.de/politik/hintergrund-aktuell/160780/asylkompromiss-24-05-2013 (Zugriff am 15.1.2018).

Children \& Society (2007) vol.21, issue 4, The International Journal Of Childhood And Children's Services, National Children's Bureau, Oxford : Wiley-Blackwell

Committee on the Rights of the Child (2013): General comment No. 14 (2013) on the right of the child to have his or her best interests taken as a primary consideration (art. 3, para. 1), https://www2.ohchr.org/English/bodies/crc/docs/GC/ CRC_C_GC_14_ENG.pdf (Zugriff 14.10.2018).

Deutsche Liga für das Kind (2016): Wir sind da! Kinder aus Krisenregionen. Eine Herausforderung für unsere Gesellschaft. Dokumentation der Jahrestagung 2016. In: Frühe Kindheit. Die ersten sechs Jahre. Zeitschrift der Deutschen Liga für das Kind in Familie und Gesellschaft e. V.

Deutsches Jugendinstitut (2016): Flüchtlingskinder in der Kindertagesbetreuung. Ergebnisse der DJI-Kita-Befragung „Flüchtlingskinder“ zu Rahmenbedingungen und Praxis im Frühjahr 2016. Autor*innen: Benjamin Baisch, Kilian Lüders, Christiane Meiner-Teubner, Birgit Riedel, Antonia Scholz, https://www. dji.de/fileadmin/user_upload/bibs2017/Fluechtlingskinder_in_Kindertagesbetreuung.pdf (Zugriff 12.6.2018).

Deutsches Kinderhilfswerk (2017): Pressemitteilung vom 19.6.2017, https://www. dkhw.de/presse/pressemitteilungen/presse-details/repraesentative-umfrage-imauftrag-des-deutschen-kinderhilfswerkes-zum-weltfluechtlingstag-2017-integ/ (Zugriff 23.8.2018).

Emcke, Carolin (2016): Gegen den Hass. Frankfurt a. M.: Fischer.

Engel, Anne Marlen (2018): Flüchtlingslager als Orte des Ausnahmezustands? Eine rechtssoziologische Untersuchung. Assoziierte Forschung an der HU Berlin, https://www.rewi.hu-berlin.de/de/lf/ls/bae/assoziierte-Forschung/annemarlen-engler (Zugriff am 17.8.2018).

Fegert, Jörg. M., Diehl, Claudia, Leyendecker, Birgit \& Hahlweg, Kurt (2017): Aus Kriegsgebieten geflüchtete Familien und ihre Kinder: Entwicklungsrisiken, Behandlungsangebote, Versorgungsdefizite. Kurzgutachten des Wissenschaftlichen Beirats für Familienfragen beim Bundesministerium für Familie, Senioren, Frauen und Jugend. Berlin. 
Genfer Flüchtlingskonvention (1951): Abkommen über die Rechtstellung von Flüchtlingen. https://www.fluechtlingskonvention.de/ (Zugriff 11.6.2018).

Gesetz zu dem Haager Übereinkommen vom 19. Oktober 1996 über die Zuständigkeit, das anzuwendende Recht, die Anerkennung, Vollstreckung und Zusammenarbeit auf dem Gebiet der elterlichen Verantwortung und der Maßnahmen zum Schutz von Kindern. Stand 25. Juni 2009 (KSÜ).

GGUA (2016): Bleibeperspektive: Kritik einer begrifflichen Seifenblase. GGUAgemeinnützige Gesellschaft zur Unterstützung Asylsuchender e. V., https:// ggua.de/fileadmin/downloads/tabellen_und_uebersichten/bleibeperspektive.pdf (Zugriff 1.4.2019).

Giner, Clotilde (2007): The Politics of Childhood and Asylum in the UK. In: Children \& Society, 21, Nr. 4 (2007), S. 249-260.

González Méndez de Vigo, Nerea, Schriefers, Silvia \& Hager, Nina (2017): Arbeitshilfe zur Beantragung der Kostenübernahmen von Therapie mit minderjährigen Geflüchteten und jungen Volljährigen, http://www.baff-zentren.org/wpcontent/uploads/2017/02/BumF_BAfF-Arbeitshilfe-Therapie-Jugend.pdf (Zugriff 14.10.2018).

Goodman, Simon (2007): Constructing Asylum Seeking Families. In: Critical Approaches to Discourse Analysis Across Disciplines, 1 (1) (2007), S. 36-50.

Jugend- und Familienministerkonferenz (JFMK) (2016): Öffentliches Protokoll 2./3. Juni 2016, https://www.jfmk.de/pub2016/JFMK_2016_Protokoll.pdf (Zugriff 16.10.2017).

Laclau, Ernesto \& Mouffe, Chantal (1985): Hegemony and Socialist Strategy: Towards a Radical Democratic Politics. London: Verso.

Lewek, Mirjam \& Naber, Adam (2017): Kindheit im Wartezustand. Studie zur Situation von Kindern und Jugendlichen in Flüchtlingsunterkünften in Deutschland. Köln: UNICEF.

Liebel, Manfred (2018): Paternalismus im Namen des Kindeswohls. Auf dem Weg zu einer Provinzialisierung der Kinderrechte? Rezensionsaufsatz. Friederike Wapler (2015): Kinderrechte und Kindeswohl. Eine Untersuchung zum Status des Kindes im Öffentlichen Recht, Heft 76/2018. In: Sozialwissenschaftliche Literaturrundschau, Heft 76, 2018, S. 64-72.

Liebel, Manfred (2018): Welfare or Agency? Children's Interests as Foundation of Children's Rights. In: International Journal on Children's Rights, 26 (2018), S. $1-31$.

Liebel, Manfred (2017): Kinderbewegungen im Globalen Süden als Bürgerschaft von unten. In: Silke Jakob (Hrsg.): Engagierte Jugendliche in der Gesellschaft. Bürgerschaft und Engagement in einer globalisierten Welt. Opladen: Barbara Budrich, 2017, S. 43-68.

Liebel, Manfred, Nnaji, Ina \& Wihstutz, Anne (2008): Abschlusserklärung des Ersten Welttreffens arbeitender Kinder, Kundapur 1996. In: diess. (Hrsg.): Kinder. Arbeit. Menschenwürde. Internationale Beiträge zu den Rechten arbeitender Kinder. Frankfurt a. M./London: IKO, S. 431.

Meysen, Thomas, Beckmann, Janna \& González Méndez de Vigo, Nerea (2016): Flüchtlingskinder und ihre Förderung in Tageseinrichtungen und 
Kindertagespflege, Rechtsexpertise im Auftrag des Deutschen Jugendinstituts, https://www.dji.de/fileadmin/user_upload/bibs2016/Meysen_et_al_expertise_kitazugang_fluechtlingskinder_2016.pdf (Zugriff 13.10.2017).

Meysen, Thomas, Beckmann, Jana \& Nerea Gonzalez Mendez de Vigo (2016): Zugang begleiteter ausländischer Kinder zu Leistungen der Kinder- und Jugendhilfe nach der Flucht. In: NVwZ 7, S. 427-431.

Nonhoff, Martin (2007): Diskurs, radikale Demokratie, Hegemonie - Einleitung. In: ders. (Hrsg.): Diskurs - radikale Demokratie - Hegemonie. Zum politischen Denken von Ernesto Laclau und Chantal Mouffe. Bielefeld: Transkript, S. 724.

Parusel, Bernd (2017): Asylpolitische Restriktionen und ihre Folgen am Beispiel Schweden, https://fluechtlingsforschung.et/asylpolitische-restriktionen- undihre-folgen-am-beispiel-schweden/ (Zugriff 21.8.2018).

Peschel-Gutzeit, Lore (2015): Kinderrechte ins Grundgesetz? Pro: Mehr als eine abstrakte Forderung: Kindergrundrechte würden die gesellschaftliche Bedeutung der Kinder endlich klar ausdrücken. In: DJI Impulse: Kinderrechte zwischen Anspruch und Wirklichkeit. Warum die Umsetzung ein schwieriger Prozess ist, Ausgabe 3, S. 8.

Pries, Ludger (2016): Migration und Ankommen. Die Chancen der Flüchtlingsbewegung. Frankfurt a. M./New York: Campus.

Pro Asyl (2015): Aussetzung des Familiennachzugs wird Frauen und Kinder in den Tod schicken, https://www.proasyl.de/news/aussetzung-des-familiennachzugswird-frauen-und-kinder-in-den-tod-schicken/ (Zugriff 14.10.2018).

Reckwitz, Andreas (2006): Ernesto Laclau. Diskurse, Hegemonien, Antagonismen. In: Moebius, Stephan \& Quadflieg, Dirk (Hrsg.): Kultur. Theorien der Gegenwart. Wiesbaden: VS Verlag für Sozialwissenschaften, S. 339-349.

Richtlinie 2013/33/EU des Europäischen Parlaments und des Rates der Europäischen Union vom 26. Juni 2013 zur Festlegung von Normen für die Aufnahme von Personen, die internationalen Schutz beantragen, Abl. L180/96 vom 29. Juni 2013.2.

Riedel, Lisa \& Schneider, Gerald (2017): Dezentraler Asylvollzug diskriminiert: Anerkennungsquoten von Flüchtlingen im bundesdeutschen Vergleich, 20102015. In: Politische Vierteljahresschrift 58 (1), S. 21-48, https://www.nomoselibrary.de/10.5771/0032-3470-2017-1-23/dezentraler-asylvollzug-diskriminiert-anerkennungsquoten-von-fluechtlingen-im-bundesdeutschen-vergleich2010-2015-jahrgang-58-2017-heft-1?page=1 (Zugriff 17.5.2017).

Schäfer, Alfred (2016): Hegemonie. In: Mecheril, Paul (Hrsg.): Handbuch Migrationspädagogik. Weinheim: Beltz, S. 90-105.

Schuster, Anna-Lena (2014): Ein besonderes Bedürfnis - Das Beurteilungserfordernis des Artikel 22 der Aufnahmerichtlinie, http://www.asyl.net/fileadmin/user_upload/beitraege_asylmagazin/Beitraege_AM_2014/AM2014_7_8_beitragschuster.pdf (Zugriff 24.2.2017).

Spiegel (2017): Mehrheit der Deutschen gegen gleiche Rechte für Flüchtlingskinder, http://spiegel.de/lebenundlernen/schule/fluechtlinge-mehrheit-der- 
deutschen-gegen-gleiche-rechte-fuer-fluechtlingskinder-a-1152883.html (Zugriff 20.6.2016).

Süddeutsche Zeitung (2018): SPD will beim Familiennachzug nachverhandeln. 18. Januar 2018, https://www.sueddeutsche.de/politik/migration-fluechtlinge-asylpolitik-spd-familiennachzug-1.3831141 (Zugriff 14.10.2018).

SVR (2017): Fakten zur Asylpolitik. Kurz und Bündig. 1. Halbjahr 2017, https:// www.svr-migration.de/wp-content/uploads/2017/08/SVR_Fakten_zur_Asylpolitik.pdf (Zugriff 15.1.2018).

SVR (2018): Fakten zur Asylpolitik 2017. https://www.svr-migration.de/wp-content/uploads/2018/05/SVR_Fakten_zur_Asylpolitik.pdf (Zugriff 19.6.2018).

UNICEF (о. J.): Konvention über die Rechte des Kindes, https://www.unicef. de/blob/9364/a1bbed70474053cc61d1c64d4f82d604/d0006-kinderkonventionpdf-data.pdf (Zugriff 14.6.2017).

Wapler, Friederike (2015a): Kinderrechte ins Grundgesetz? Starke Kinderlobby statt Gesetzeslyrik: Was Kindern wirklich hilft, ist eine Politik, die ihre bestehende Rechte konsequent umsetzt. In: DJI Impulse „Kinderrechte zwischen Anspruch und Wirklichkeit. Warum die Umsetzung ein schwieriger Prozess ist" Ausgabe 3, S. 9.

Wapler, Friederike (2015b): Kinderrechte und Kindeswohl. Eine Untersuchung zum Status des Kindes im Öffentlichen Recht. Tübingen: Mohr Siebeck.

Wendel, Kay (2014): Unterbringung von Flüchtlingen in Deutschland. Regelungen und Praxis der Bundesländer im Vergleich. Hrsg. vom Förderverein ProAsyl e. V., Frankfurt am Main, https://www.proasyl.de/wp-content/uploads/2014/09/ Laendervergleich_Unterbringung_2014-09-23_02.pdf (Zugriff 21.8.2018).

Wihstutz, Anne (2018) „Grundbegriff Kindheit“ - Eine soziologische Perspektive. In: Rademacher, Sandra \& Kleepage-Niepage, Andrea (Hrsg.): Kindheit Sammelband „Kindheits- und Jugendforschung in der Kritik“. Wiesbaden: VS, S. 91-110.

Wihstutz, Anne (2017) From Objects of care to citizens- young carers' citizenship. In: Warming, Hanne/Fahnoe, Kristian (eds). Lived Citizenship on the Edge of Society. Rights, Belonging, Intimate Life and Spatiality. Cham: Palgrave Macmillan - Springer Nature, S. 175- 198.

Wissenschaftlicher Dienst (2016): Deutscher Bundestag WD 2-3000 - 026/16, Vereinbarkeit der Regelungen des Asylpakets II betreffend die Aussetzung des Familiennachzugs für unbegleitete minderjährige Flüchtlinge mit der UN-Kinderrechtskonvention (UNKRK), https://sehrgutachten.de/bt/wd2/026-16-vereinbarkeit-der-regelungen-des-asylpakets-ii-betreffend-die-aussetzung-des-familiennachzugs-fuer-unbegleitete.txt)quelle, (Zugriff 14.6.2017).

Zeit online (2017): Mehr als 65 Millionen Menschen weltweit auf der Flucht. 19. Juni 2017, http://www.zeit.de/gesellschaft/zeitgeschehen/2017-06/fluechtlingesyrien-zahl-rekordhoch-unher (Zugriff 15.1.2018).

Zeit online (2018): Deutschland verstößt bei Familiennachzug wohl gegen EURecht. 12. April 2018, https://www.zeit.de/politik/deutschland/2018-04/familienzusammenfuehrung-fluechtlinge-minderjaehrige-eugh-urteil (Zugriff 14.10. 2018). 


\title{
Ethisch-reflexive Auseinandersetzungen im Forschungsprozess
}

\author{
Hoa Mai Trần
}

Ethische Fragen sind essentieller Bestandteil wissenschaftlicher Auseinandersetzungen und unabdingbares Gütekriterium von Forschung. Forschungen sind eingebettet in gesellschaftliche Diskurse und finden nicht im „luftleeren“ Raum statt, sondern in einem gesellschaftlichen und dynamischen Kontext. ${ }^{1}$ Vor diesem Hintergrund stellt das Forschungsfeld besondere Anforderungen an die konkrete Ausgestaltung von Forschung und eröffnet eine kritische Auseinandersetzung mit vielseitigen ethischen Fragestellungen und ihren Ambivalenzen. Dieser Beitrag fußt auf den Erfahrungen und Überlegungen des durchgeführten qualitativ-ethnografischen Forschungsprojekts zum Alltagserleben junger Kinder in Unterkünften für geflüchtete Menschen.

Ziel dieses Beitrags ist es, Facetten der durchgeführten Forschung zu reflektieren und ihre Bedeutung für die Forschungspraxis deutlich zu machen. Die im Forschungsprozess entstandenen Irritationen und Fragen werden mit forschungsethischer Relevanz dargelegt und kontextualisiert.

Nach einer Einführung und definitorischen Annäherungen an Forschungsethik, werden verbreitete Prinzipien allgemein geltender Richtlinien als Grundlage eingeführt. Daran anschließend wird reflexive Subjektivität als wesentlicher Grundbaustein ethischer Überlegungen hervorgehoben. Im Anschluss werden Herausforderungen angesprochen, die sich aus dem Blickwinkel von Forschung in Machtverhältnissen ergeben. Entlang der Krise der Repräsentation werden Strukturen der Wissensproduktion in den Blick genommen. Ethische Grundsätze werden bezogen auf den konkreten Forschungskontext und in ihrer Ambivalenz als Dilemmata diskutiert. Anschließend wird die Rolle der Forscherin im Feld beschrieben. Aus den ethischen Spannungsverhältnissen im Feld wird die Grundannahme ,,do no harm“ is not enough““ (Hugman et al. 2011: 1271) in ihrer Konsequenz auf das Forschungsprojekt übertragen. Neben selbstkritischer Reflexion des Handelns der Forscherinnen ${ }^{2}$ und ihrer Positionierung im Feld, wird der Versuch unternommen, möglichst

1 Für die theoretische und gesellschaftliche Verankerung des Forschungsprojekts siehe den Beitrag von Wihstutz „Das Forschungsprojekt und sein Design“.

2 Es wird in diesem Beitrag in der feldbezogenen Auseinandersetzung von Forscherinnen gesprochen, da sich im Forschungsteam alle Personen als weiblich identifizieren und sich der Beitrag grundlegend auf ihre Erfahrungen im Forschungsprozess bezieht. 
vielseitige Mehrwerte für und mit den Forschungsteilnehmer*innen sicherzustellen. Dies kann als engagierte Forschung verstanden werden. Die Ausführungen dieses Beitrags erheben keinen Anspruch auf Vollständigkeit und sind vielmehr als Anregungen zu verstehen, sich mit Forschungsethik in verschiedenen Aspekten auseinanderzusetzen und damit zu einer verantwortungsbewussten und reflexiven Forschungspraxis beizutragen.

\section{Einführung in Forschungsethik}

Mit Forschungsethik in der Sozialforschung werden in diesem Beitrag primär Prinzipien und Regeln verbunden, welche nach der Gestaltung der Beziehungen zwischen der forschenden Person und der in der Untersuchung einbezogenen Menschen (Forschungsteilnehmer*innen) fragen (vgl. Hopf 2004: 589 ff.). Ethische Prinzipien sind durch rechtliche Anforderungen (z. B. Datenschutzgesetze auf Bundes- und Landesebene), Persönlichkeitsrechte, theoretische, methodologische und politische Fragen von Wissenschaft unter anderem in Bezug zu handlungsorientierter Ethik und Ethikkodizes gekennzeichnet (vgl. von Unger et al. 2014: 2). Zusammenfassend sind es folgende oft benannte Grundprinzipien und Grundsätze, die gegenüber den Forschungsteilnehmer*innen als relevant gelten (vgl. Alderson \& Morrow 2011: 17, 31, 100 ff.; DGfE 2010; DGS 2014; DGV 2009; Guillemin \& Gillam 2004: 270; Hopf 2004: 589-597; Hugman et al. 2011: 1275; von Unger et al. 2014: 2, 20):

- die Einhaltung der Persönlichkeitsrechte genauso wie Verantwortung gegenüber individuellen und kollektiven Interessen durch rechtliche Orientierungen (z. B. an Menschenrechten und Kinderrechten);

- die Sicherstellung von Anonymität und Vertraulichkeit als Wahrung der Integrität der Forschungsteilnehmer*innen, welche ebenfalls die Informationsweitergabe oder auch den kontrollierten Datenzugang im Sinne des Datenschutzes betrifft;

- das Verfahren der informierten Einwilligung durch die Sicherung von Freiwilligkeit der Teilnahme und das Recht zur freien Entscheidung darüber wie auch die Transparenz in der Kommunikation von Zielen, Inhalten und Methoden des Forschungsvorhabens gegenüber Forschungsteilnehmer*innen;

- das Prinzip der Nichtschädigung durch Risikoabwägung als die Aufklärung und Antizipation möglicher Risiken und negativer Konsequenzen für Individuen oder Gruppen, die keinen Nachteilen ausgesetzt werden. Ziel ist es, Schäden möglichst zu vermeiden, gering zu halten oder, falls aufgetreten, zu minimieren. 
Diese allgemein formulierten Prinzipien dienen maßgeblich als Richtlinien. Sie bedürfen in ihrer Umsetzung einer Kontextualisierung als „fallbezogene Interpretation" (von Unger et al. 2014: 18) und werden im Beitrag aus empirischer Perspektive verhandelt. Eine mögliche Differenzierung stellt die Aufgliederung von prozeduraler und praktischer Ethik dar. Dabei bezieht sich prozedurale Ethik auf formale Grundsätze und gilt als relativ kontextunabhängig. Sie ist durch Richtlinien gekennzeichnet, die innerhalb von Gremien, Kommissionen, Begutachtungen und Kodizes festgelegt sind. Fragen praktischer Ethik ergeben sich aus den Herausforderungen der Forscher*innen in der Forschungspraxis. ${ }^{3}$ Eine Reduzierung von Forschungsethik auf ethische Richtlinien oder auch rechtliche Anforderungen kann durch ausbleibende Kontextsensibilität als Verengung gelten. Forschungsethik hat „in erster Linie etwas mit sozialer Verantwortung zu tun" (von Unger et al. 2014: 32), welche zu jedem Zeitpunkt der Forschung zum inhaltlichen Gegenstand der Auseinandersetzung werden kann:

„Empirisch Forschende müssen in diesem Kontext Entscheidungen über Verfahren und Prozesse treffen, die weitreichende Konsequenzen für das Leben anderer Menschen nach sich ziehen können. Diese Entscheidungen verlangen Abwägungen, Begründungen und damit eine Reflexivität, die den Kern dessen ausmacht, was [...] unter Forschungsethik verstanden wird" (von Unger et al. 2014: 16).

Forschungsethische Fragen verlangen mehr nach Abwägungen, weniger nach einer Vorgabe spezifischer Positionen oder einer vorgegebenen Handlungsmoral und -vorlage. ${ }^{4}$ Das Handeln ${ }^{5}$ der Forscher*in wird als angemessen oder unangemessen im konkreten Kontext begründet, entsprechend realisiert und nachvollziehbar gemacht. Forschungsethik kann als selbstkritische Verantwortung der Forscher*innen und als Selbstverpflichtung betrachtet werden (von Unger et al. 2014: 2). ${ }^{6}$ Die angesprochene Kontextabhängigkeit bezieht

3 Sie entstehen aus dem konkreten Forschungskontext und können auch als Mikroethik verstanden werden (vgl. Block et al. 2013: 70; Guillemin \& Gillam 2004: 261, 269).

4 Dabei ist die philosophische Herkunft von Ethik als die Auseinandersetzung mit dem „guten Leben“ an normative Referenzsysteme gebunden (Brumlik 2017: 18). Bezogen auf Forschungsethik kann äquivalent die Bearbeitung und Aushandlung ,guter“ Forschung eingeschlossen werden.

5 Unter Forschungshandeln wird das Tun, Dulden oder Unterlassen als Aktivität der Forscher*in im gesamten Forschungsprozess von Fragestellung zu Forschungsdesign, Erhebung und Auswertung bis hin zur Publikation und Öffentlichkeitsarbeit eingeschlossen.

6 Diese lassen sich je nach Forschungsansatz in qualitativer und/oder qualitativer Forschung und jeweiligen Themengebiet auch verschieden stellen. Sie sind mit ihrer Antizipation und Anwendung im Konkreten als voraussetzungsvoll zu betrachten und an verschiedenen Anforderungen und Kompetenzen gebunden. 
sich als ,relationale Ethik“7 (Kaukko et al. 2017: 16) auf die Beziehungen zwischen den Forscher*innen und dem Forschungsfeld samt beteiligter Akteur*innen. Sie schließt die Auseinandersetzung mit gesellschaftlich-politischen Diskursen ein. Gegenstand reflexiver Überlegungen können bereits die Themenwahl, die Zielsetzung des Vorhabens oder das Forschungsdesign betreffen. Weiterhin kann das Ad-hoc-Handeln im Feld, die Erhebungsphase, die Datengenerierung und -auswertung bis hin zur Publikation und Verwertbarkeit der Forschungsergebnisse Bestandteil ethischer Überlegungen sein.

\section{Subjektivität und Interaktivität der Forscher*in im Feld}

In der Ethnografie werden Forscher*innen im Feld zum Erhebungsinstrument. Sie erleben das Geschehen leibhaftig mit allen Sinnen (vgl. Stodulka 2014: 180). Die forschende Person besitzt soziale, kulturelle, historische als auch äuBerliche und persönliche Merkmale und tritt mit diesen in das Forschungsfeld ein. Diese Eigenschaften treten auch mit den beteiligten Akteur*innen in Wechselwirkung (vgl. Breuer 2004: Abs. 3, 22). In der Praxis des hier vorgestellten Forschungsprojekts stehen die Perspektiven der jeweiligen Forscherin in Beziehung zu persönlichen Merkmalen und ihrem wissenschaftsdisziplinären Hintergrund. Im Forschungsteam arbeiteten Sozialanthropologinnen, Ethnologinnen, Sozialwissenschaftlerinnen, Sozialarbeiterinnen und Kindheitspädagoginnen interdisziplinär und komplementär in Forschungstandems zusammen. Die Zusammenstellung der Tandems von weißen ${ }^{8}$ Forscherinnen und Forscherinnen of Color ${ }^{9}$ ist im Feld bedeutsam geworden. Forscherinnen of Color wurden beispielsweise nach ihrer Herkunft gefragt und auch den Bewohner*innen der Unterkunft zugeordnet. Eine solche Klassifizierung erfuhren weiße Forscherinnen entlang ,,natio-ethno-kultureller Zugehörigkeitsordnungen“"(Castro Varela \& Mecheril 2010: 41) nicht. Als mögliche Einschrän-

„Relational ethics mean that the process of taking and giving back can be blurry; both the refugee child and the researcher enter a process of giving, taking, teaching and learning while seeking appropriate, supportive and productive ways of working towards a shared goal" (Kaukko et al. 2017: 19).

8 Der Begriff weiß bezieht sich auf die soziale, politische und gesellschaftliche Position entlang rassistischer Strukturen als herrschende Normalität, die historisch begründet und mit Privilegien verbunden ist (Piesche \& Arndt 2011: $192 \mathrm{f}$.).

9 Abgeleitet von der Begrifflichkeit People of Color, welche sich auf Menschen bezieht, die Fremdzuschreibungen in einer weißen Dominanzgesellschaft erleben und als nicht zugehörig markiert werden. Ihre Position wird als minorisiert und durch Rassismuserfahrungen geprägt beschrieben (Ha et al. 2007: 12). 
kung kann auch der Umstand gedeutet werden, dass die Forscherinnen selbst keine Fluchterfahrung erlebt haben. Schlussfolgernd beforschten sie eine Lebensrealität, die nicht ihrer eigenen entspricht. Der Erfahrungs- und Vergleichshorizont als Nichtgeflüchtete kann auch die Gefahr des Missverstehens und -interpretierens in sich tragen (vgl. Block et al. 2013: 71). Weiterhin trug eine intersektionale Zusammensetzung zur Sensibilität gegenüber Diskriminierungen bei, denen Forscherinnen und Akteur*innen im Feld ausgesetzt waren. Die komplementär angelegte Analyse der Zusammensetzung von Forschungsteams kann in dieser Hinsicht nicht nur in der Erhebung, sondern ebenfalls in der Auswertung als erkenntnisförderlich betrachtet werden. Die Forscherinnen protokollierten die Daten beispielsweise durch die Beschreibung von Personen. Dabei führte die (Nicht-) Benennung wahrgenommener äußerlicher oder zugeschriebener Merkmale als einhergehende sozial hergestellte Differenz im Forschungsteam zu Diskussionen. So herrschte im Team kein klarer Konsens darüber, ob es nötig ist ein Kind als männlich oder weiblich zu beschreiben und ab wann Merkmale wie religiöse Symbole oder Hautfarbe bedeutsam werden.

Eine Forscherin assoziierte mit der Verwendung von Nummerierungen und Abkürzungen im Pseudonymisierungsprozess Praktiken in Konzentrationslagern, da der Einsatz von Ziffern an Stelle des Namens von Personen als entmenschlichend und objektivierend wahrgenommen wurde. Temporär verwendete Pseudonyme wurden daher durch Selbstpositionierungen der Akteur*innen im Feld oder ihren institutionellen Funktionen ersetzt.

Die Forscherin tritt nicht nur in das Feld ein, sondern ihre Präsenz, ihr Auftreten und ihre äußerlichen Merkmale lösen im Gegenüber Reaktionen aus. Diese Interaktivität ist als wechselseitige Bezugnahme mit Akteur*innen begreifbar. Dies bezieht sich nicht nur auf zwischenmenschliche Beziehungen, sondern vermittelt sich darüber hinaus durch Erhebungsmethoden. Forschung kann als Intervention betrachtet werden, da sie das soziale Geschehen verändert. Eltern reagierten abgeschreckt auf die Tätigkeit des Protokollierens der Forscherinnen, so dass von weiteren Notizen zu Beginn des Kennenlernens abgesehen wurde. Bei Kindern stießen Notizen teilweise auf großes Interesse, wobei Forscherinnen aufgefordert wurden das Geschriebene vorzulesen. Kinder kommentierten die Notizen, ergänzten Punkte, die ihnen wichtig waren oder sie malten etwas dazu und nahmen dadurch Einfluss auf die Datenerhebung. ${ }^{10}$ Die Präsenz der Forscherin im Feld durch teilnehmende Beobachtung ist bereits als Interaktion und Intervention im Mikrokontext zu verstehen. Gleichzeitig hatten Akteur*innen im Feld Einfluss auf das Handeln der Forscherinnen. Sie konnten entscheiden an welchen Orten (nicht) geforscht wurde und unter welchen Bedingungen, Möglichkeiten und Grenzen die geplante

$10 \mathrm{Zu}$ methodisch und methodologischen Aspekten und Anforderungen in der Forschung mit und über Kinder und Kindheiten und der konkreten Ausgestaltung siehe den Beitrag von Wihstutz „Das Forschungsprojekt und sein Design“. 
Forschung stattfand. So rannten Kinder in andere Räume, woraufhin die Forscherin folgen musste. Sie verweigerten oder forderten sich Tätigkeiten ein und trugen maßgeblich zur Forschungssituation bei. Eltern bestimmten auch über das Gesprächsthema, den Ort des Aufenthalts sowie die stattfindenden Aktivitäten im Feld. Das Handeln im Feld war geprägt von Kreativität, Flexibilität und der Adaption von Methoden an verschiedene Forschungsteilnehmer*innen und situativen Gegebenheiten. Nicht zuletzt waren die konkreten räumlichen Bedingungen und institutionellen Reglementierungen verschiedener Unterkünfte für die Erhebungsphase entscheidend. Festzuhalten ist, dass der Standort und die Perspektive der Forscherin subjektgebunden sind. Jede Erkenntnis konstituiert sich zeitlich gebunden, räumlich verortet und wird durch wechselseitige soziale Bezugnahme durch soziale Akteur*innen hervorgebracht.

Die Anerkennung des Subjektstatus der Forscherinnen, genauso wie der Akteur*innen im Feld, und der daran anschließenden Interaktivität und Standortgebundenheit ist grundlegend, um den Prozess der Hervorbringung von Wissen als Konstruktionsprozess von Forschung nachzuvollziehen. Diese Beziehungen sind darüber hinaus bedeutsam für die ethische und reflexive Auseinandersetzung mit der eigenen Forschungspraxis (vgl. Guillemin \& Gillam 2004: 262). Die Reflexion über die eingenommene(n) Rolle(n), konkreten Verhaltensweisen, methodischen Entscheidungen und Interpretationsfolien der Forschenden haben das Potential, Selbstverständliches der eigenen Praxis zu hinterfragen, Haltungen sichtbar zu machen sowie Probleme, Irritationen und Befindlichkeiten zu thematisieren. Der Erfahrungs- und Perspektivenaustausch ist Voraussetzung, um einen Raum zu schaffen, in dem über ethische Fragen in der Forschungspraxis nachgedacht werden kann (vgl. Christensen \& Prout 2002: 477). Der Austausch innerhalb der Forschungstandems im Forschungsteam und mit Akteur*innen im Feld erwies sich als bedeutsam, um feldbezogenen Anforderungen zu begegnen, zu einer Rollenklärung beizutragen und eigenes Handeln kritisch zu reflektieren. Im Verlauf des Forschungsprojekts wurden regelmäßig interdisziplinäre Forschungswerkstätten zur Diskussion besucht sowie Supervisionssitzungen in Einzel-, Tandem- und Teamkonstellationen in Anspruch genommen.

\section{Forschen in Machtverhältnissen}

Neben verschiedener natio-ethno-kultureller Zugehörigkeiten im Forschungsteam sind weitere soziale Positionierungen beispielsweise entlang von Fluchtstatus (Geflüchtete - Nichtgeflüchtete), Generation (Kinder - Erwachsene), Geschlecht (männlich - weiblich - nicht binär) als kategoriale Bestimmungen entlang gesellschaftlicher Ungleichheiten forschungsethisch relevant. Diese 
sind durch weitere Differenzmerkmale wie sozialer Herkunft, gesundheitlicher Verfassung, nationaler Zugehörigkeiten erweiterbar. Die sozialen Positionierungen sind jenseits ihrer Dichotomien und als verschränkt zu verstehen. Ihre Differenzlinien werden durch hegemoniale Ausprägungen als Herrschaftsund Machtverhältnisse wirksam (vgl. White \& Bushin 2009: 328 f.). „Kind sein" als sozial-konstruierte Kategorie ist eine gesellschaftlich hergestellte generationale Unterscheidung. Diese impliziert die Abweichung von der Norm („Erwachsen sein“). Kindern wird fehlende Mündigkeit und Entwicklungsbedürftigkeit zugeschrieben. Auf Grund ihres eingeschränkten rechtlichen Status sind sie in hohem Maße von ihren Eltern abhängig. Durch die Kombination sozialer Kategorien, wie junge Menschen mit Fluchterfahrung, verschränken sich soziale Positionen. In ihrer Konsequenz sind sie Ausdruck von Benachteiligung und Privilegierung, in denen ex- und inkludierende Mechanismen wirksam werden. Damit werden Teilhabemöglichkeiten benachteiligten Personengruppen verwehrt, über die bevorzugte Personengruppen verfügen. Geläufige Diskriminierungsformen sind als machtkritische Lesart beispielsweise Sexismus, Klassismus, Rassismus, Ableismus, Linguizismus oder Adultismus, die nicht nur als individuelle Erfahrungen, sondern als komplexe, dynamische und historisch gewachsene Machtverhältnisse in der Gesellschaft verstanden werden können ${ }^{11}$.

11 Sogenannte ,-ismen“ drücken Diskriminierungsmechanismen aus. Diese sind an Praktiken der Abwertung, Benachteiligung und Ausgrenzung von Menschen(gruppen) definierbar und führen umgekehrt zur Privilegierung dominanter Menschen(gruppen). Nach dem Allgemeinen Gleichbehandlungsgesetz werden Merkmale wie ethnische Herkunft, Geschlecht und Religion, sexuelle Identität, Alter angesprochen. Diskriminierungsformen werden individuell und in Interaktionen erlebt und finden sich auch institutionell und strukturell wieder. Sie sind Ausdruck dauerhafter Benachteiligung, sozialer Ungleichheit und Unterdrückung und haben historische Bezüge (vgl. Gomolla 2016: 74ff.). Sexismus bezeichnet die differente Bewertung und Behandlung von Menschen auf Grund ihres tatsächlichen oder vermuteten Geschlechts - analog zu Klassismus wird die Diskriminierung auf Basis der sozialen Herkunft nach Einkommen, Berufsstand und Bildungsstatus getroffen. Rassismus bezeichnet die Unterscheidungen auf Grund von Nationalität, Kultur, Hautfarbe und ethnischen Zugehörigkeit beispielsweise in Bezug zu Sinti und Roma. Dabei verschränkt sich auch Religiöse Zugehörigkeit (z.B. Antimuslimischer Rassismus, Antisemitismus) mit rassistischen Klassifizierungskategorien. Ableismus bezieht sich auf körperliche und geistige Fähigkeiten von Menschen, wobei Nicht-Behinderung als Norm gilt. Linguizismus gilt ähnlich für die Auf- und Abwertung gegenüber Sprachen und Sprecher*innen von dominierenden Sprachen gegenüber Minderheitensprachen. Auch kann der Umgang mit Mehrsprachigkeit als auch Dialekte und Akzente darunter gefasst werden. Adultismus beschreibt die Machtungleichheit zwischen Kindern und Erwachsenen entlang generationaler Ordnung und kann entlang des Alters auch gegen ältere Menschen verstanden werden. Jede Diskriminierungsform hat eigene Mechanismen und Spielarten. Sie verbindet im Wesentlichen die Herstellung von Differenz als Unterscheidung. Die Auf- und Abwertung und damit einhergehende (legitime) Ungleichbehandlung in Verbindung mit Machtverhältnissen auf gesellschaftlich-historischer Gewachsenheit. 
In der Forschung mit Kindern mit Fluchterfahrung wird von politisch und sozial vulnerablen Personen gesprochen, welche häufig Mehrfachbenachteiligungen erleben und auch in asyl- und aufenthaltsrechtlich (strukturell) bedingten prekären Verhältnissen leben müssen. Fluchterfahrungen, Brüche in sozialen Beziehungen, limitierter Zugang zu gesellschaftlichen Ressourcen und rechtliche Sonderstellungen führen zu vielseitigen Diskriminierungen in der Aufnahmegesellschaft, was als strukturelle und institutionalisierte Gewalt konzeptionalisiert werden kann, welche auch im alltäglichen Leben Ausdruck finden. So sind die „beforschten“ Lebensverhältnisse im Projekt selbst Ausdruck globaler, nationaler und lokaler Macht- und Dominanzverhältnisse, welche auch die Forschungsbeziehung betrifft (Cabot 2016: 5). In der Analyse der Verhältnisse in den Unterkünften im Ergebnisteil der Forschung wird deutlich, dass bereits das Leben der Familien in einer Unterkunft Zeugnis von struktureller Gewalt, Fremdbestimmung und einer benachteiligten Lebenslage ist ${ }^{12}$ : Das Ideal auf Augenhöhe zu forschen wurde zwar individuell angestrebt, kann aber bereits als strukturell verunmöglicht problematisiert werden. Die Suche nach einem Mehr an Partnerschaftlichkeit in der Beziehung zu den Akteur*innen mit Fluchtstatus ist ambivalent und gekennzeichnet durch ungleich verteilte Ressourcen, Zugänge, Handlungsmöglichkeiten und Bedarfe.

Die in unserem Projekt privilegiertere Position der Forscherin und ihr Handeln werden dadurch besonders sichtbar. Dies wirft die Frage nach dem Umgang und Handeln in asymmetrisch-strukturierten Beziehungen auf. Inwiefern sind Forschungen in Gewaltverhältnisse verstrickt und was bedeutet das für die Forschungspraxis? Kann es kontextsensible und fallspezifische normativ begründete Schranken oder auch Handlungsnotwendigkeiten auf Basis eines geteilten ethischen Selbstverständnisses von Wissenschaft geben? Diese Fragen lassen sich nicht unabhängig und eindeutig beantworten und sind Gegenstand wissenschaftlicher Selbstvergewisserungen und Diskurse. Daran schlieBen sich Widersprüche und Ambivalenzen in der Rolle der Forscherin an. Bei gleichzeitiger Hilfestellung und -bereitschaft gegenüber geflüchteten Menschen entstehen Abhängigkeitsverhältnisse, die Bedürftigkeit und den Opferstatus der Betroffenen hervorbringen und reproduzieren. Durch die Lebenssituation der Betroffenen bedingt, trägt die Forscherin dazu bei, die geflüchteten Menschen in ihrer Rolle als Opfer in der Lage der Abhängigkeit zu bestärken, wenn sie die Rolle der Helfer*in einnehmen möchte. Die grundlegenden Strukturen der produzierten Bedürftigkeit der Betroffenen auf Grund ihrer Positionierung als Geflüchtete bringt durch ihre vielfach prekären Lebenssituationen ein Machtgefälle hervor. Dieses asymmetrische Verhältnis lässt die Forscherin unter anderem auch als soziale Ressource im Feld erscheinen. Es ist wahrscheinlich, dass aus der Perspektive der Geflüchteten eine implizite Erwartungshaltung, beispielsweise Hilfen zu erlangen, die Forschungsteilnahme

$12 \mathrm{Zu}$ den Auswirkungen der bürokratischen Klassifizierung „ohne Bleibeperspektive“ siehe den Beitrag von Fichtner und Trần sowie den Beitrag von Schulz-Algie. 
mitbedingt. Dies geschieht auch unabhängig davon, wie die Forscherin sich in ihrer Identität selbst betrachtet:

„Most importantly, this identity must be understood in the context of the imbalance of power between researchers and refugees in such situations. Questions of social power are embedded in all research relationsships, which is why ethics is integral to every aspect of research" (Hugman et al. 2010: 1284).

Die Identität der Forscherin steht damit nicht nur für sich, sondern ist als machtvolle soziale Position zu verstehen, welche in Beziehung zur Position der Betroffenen gesetzt werden kann. Grundlegend stellt das Bewusstsein über Machtverhältnisse, Dynamiken und Ursachen im Forschungsfeld und -prozess die Weichen für Überlegungen für eine machtkritische, verantwortungsvolle Forscherin. Die Auseinandersetzung mit Machtfragen in der Forschung oder auch von Wissenschaft ist an forschungsethische Diskussionen anschlussfähig (vgl. von Unger et al. 2014: 2). ,[R]ather than ignoring or blurring power positions, ethical practice needs to pay attention to them" (Edward \& Mauther, zitiert nach Groundwater et al. 2015: 61).

In der Forschungspraxis ist es hilfreich die Frage zu stellen, wie sich Forschung in solchen Verhältnissen gestalten lassen kann, wie machtkritisches Vorgehen aussehen kann, das über eine Bewusstheit inhärenter Machtverhältnisse auf die eigene Verstrickung verweist und praktische Konsequenzen nach sich zieht.

Auch das Forschungsteam ist nicht ausgenommen und unterliegt keinem machtfreien Raum. Entlang der unterschiedlichen Dimensionen wie Alter, akademischer Grad, soziokulturelle Herkunft und Forschungserfahrungen sind auch diese Beziehungen hierarchisch strukturiert. Die konkreten Implikationen machtkritischer Reflexion für die Praxis als auch ihr Einfluss auf die Forschungsbeziehungen werden auf einer Metaebene abstrahiert als Krise der Repräsentation verhandelt, sowie konkret entlang ethischer Dilemmata und in praktischer Konsequenz im Ausblick als engagierte Forschung im Weiteren diskutiert.

\section{Die Krise der Repräsentation}

"Closely related to issues of power, the ethical implications of how the voices and images of children are represented in research are significant ethical issues“" (Phelan \& Kinsella 2013: 86).

Die Frage der Repräsentation betrifft unmittelbar inhärente Machtverhältnisse. Mit Blick auf Feldforschung und Wissenschaft sind daran angelehnte Praktiken selbst historisch und kolonial verstrickt und an der Hervorbringung rassi- 
fizierenden Wissens und Machtverhältnissen beteiligt (Krause 2016: 13). Machtgefälle wurden mit der eigenen Forschung reproduziert: Wer forscht über wen? Wer dringt in wessen Privatsphäre? Wer erzählt wessen Geschichten? Wer kann sich den restringierten Verhältnissen der Unterkunft entziehen? Privilegierte erwachsene Forscherinnen untersuchten die Lebensrealitäten deprivilegierter und politisch vulnerabler Kinder als marginalisierte Personengruppe. Sie drangen durch die räumliche Bedingtheit von Sammelunterkünften in die Privatsphäre der Kinder, Familien und Bewohner*innen vor, um Daten zu erheben und auszuwerten. Die daraus gewonnenen Ergebnisse wurden im Anschluss mit Expertinnenstatus beispielsweise auf Tagungen präsentiert, publiziert und Forscherinnen profitieren vielseitig. Die Frage nach der gehörten „Stimme“ von Kindern liegt ebenfalls in der Erhebung, Interpretation und Auswahl erwachsener Forscherinnen. Die Krise der Repräsentation schließt das Verhältnis von Autor*innenschaft und Autorität ein, welche grundlegend aus der eigenen Position für andere spricht: „Dieser performative Akt - das ,Sprechen von' ist auch ein ,Sprechen für - als ein nicht hintergehbarer asymmetrischer Bestandteil ethnografischer Forschung“" (Breidenstein et al. 2013: 19).

Veröffentlichungen von Forschungserkenntnissen im Format einer Publikation geben beispielsweise Auskunft über Kinder und ihre Lebensverhältnisse in Sammelunterkünften. Bleibt es bei dieser Form von Formaten der Veröffentlichungen, trägt die Wissensproduktion in der ethnografischen Forschung dazu bei, das Dominanzverhältnis zwischen Forschenden und Beforschten zu reproduzieren. Die Selbstrepräsentation der an der Forschung beteiligten Personen würde andere Formate erfordern (Cabot 2016: 1). Diese Personen sind anwesend und gleichzeitig abwesend, handlungsfähig und trotzdem schweigend, da über sie gesprochen wird. Dies kann als postkoloniale Praxis dominanter Wissenskonfiguration und epistemologische Gewalt verstanden werden (ebd.: 4, 9). In der Überlegung, für wen eigentlich publiziert wird, wem das entstandene Wissen zugänglich gemacht wird und wer davon systematisch ausgeschlossen ist, wurde die Projektidee entwickelt, im Anschluss an die Forschung ein mehrsprachiges Kinderbuch im kollaborativen Prozess mit betroffenen Kindern zu erarbeiten.

Eine weitere Möglichkeit stellt Appadurai (2006) vor, indem er über das demokratisierende Vermögen von Forschung vor dem Hintergrund zunehmender Globalisierung, Ungleichheit und Teilhabe spricht. 50 Prozent der Weltbevölkerung sind aufgrund von absoluter Armut ,not even in the knowledge game" (Appadurai 2006: 168) und damit systematisch von der Teilhabe der Wissensproduktion ausgeschlossen. Er spricht sich für 30 Prozent der Weltbevölkerung aus, welche die Möglichkeit auf Elementar- und Primärbildung und eventuell auch Sekundarbildung haben. Er beschreibt die Gruppe als armutsbetroffen, politisch „schwach“, mit wenig sozial anerkanntem Kapital ausgestattet und in ökonomisch unsicheren Verhältnissen lebend. Für diese Gruppe formuliert er das Recht auf Forschung, welches Menschen befähigt, für sie 
relevante Erkenntnisse systematisch zu generieren und Forderungen sowie Handlungen für ihre Anliegen abzuleiten. Diese Verbindung von Handlungsfähigkeit und Forschungspraxis als Selbstermächtigung von marginalisierten Menschen (als Forscher*innen) fördere ihre Teilhabe an der Wissensproduktion. Er betont, dass Forschung nicht nur auf eine globale, akademische Elite fähiger Forscher*innen und Institutionen beschränkt werden solle, sondern insbesondere Menschen mit wenig sozialem, ökonomischem und politischem Kapital zugänglich gemacht werde - mit der Zielstellung, dass diese befähigt werden, ihre Forderungen und Rechte als Bürger*innen in Anspruch zu nehmen (Appadurai 2006: 167-177).

Auch Groundwater-Smith et al. (2015) verdeutlichen die Potentiale und Möglichkeiten in der Forschung mit Kindern. Kinder könnten die für sie relevanten Interessen als Forschungsthema formulieren und würden durch Trainings mit Forschungsfertigkeiten ausgestattet. Sie würden in allen Phasen des Forschungsprozesses im Sinne eines kollaborativen und partizipativen Vorgehens einbezogen (Groundwater-Smith et al. 2015: 56-59). Sie haben damit die Möglichkeit sich Forschungstechniken anzueignen und sich für ihre eigenen beziehungsweise kollektiven Interessen einzusetzen. In der rechtebasierten Forschung mit Kindern wird darüber hinaus ,the right to be properly researched" konstatiert. Kinder werden als Rechtssubjekte adressiert und die Prinzipien der UNKRK auch im Kontext von Forschung angewendet. Beispielsweise mit dem Wohl des Kindes, welches laut Artikel 3 in allen Maßnahmen die Kinder betreffen (dem Forschungsprozess eingeschlossen), Artikel 12 als dem Recht des Kindes auf die Berücksichtigung und Äußerung seines Willens und daran anlehnend Artikel 13 als dem Recht auf Information der UNKRK als forschungsrelevant benannt (Bessell et al. 2017: 222). Darunter zählen auch diverse Schutzrechte, wie das vor Diskriminierung. Diese Ansätze bieten eine interessante Alternative, um machtkritisch und teilhabeorientiert im Forschungsprozess vorzugehen und erinnern unter anderem an Aktionsforschung. Im Projekt wurden von Forscherinnen vor allem Fragen nach Methoden diskutiert. Gerade in machtvoll durchdrungenen Verhältnissen war es naheliegend die Kompetenzen und Autonomie von Kindern methodisch aufzugreifen und im Mikrokontext mitunter zu ermöglichen. Die Kinder erlebten viele alltägliche Restriktionen und Ressourcenknappheit, weswegen die Anerkennung und Sichtbarmachung ihrer Handlungsfähigkeit und Akteur*innensschaft besondere Bedeutung bekam. Es wurde versucht verschiedene Beteiligungsmöglichkeiten im Forschungsprozess zu schaffen, die an den Interessen und Bedürfnissen der Kinder ausgerichtet wurden (vgl. Block et al. 2013: 69; Mackenzieh et al. 2007: 311; Phelan \& Kinsella 2013: 85; Punch 2002: 325). 


\section{Ethische Spannungsverhältnisse und Dilemmata entlang forschungsethischer Grundsätze}

Der Forschungskontext in Unterkünften für geflüchtete Menschen stellt die Forschung vor besondere Herausforderungen. Erfahrungen im Zugang, der Einwilligung, Vertraulichkeit und vor allem Schadensabwägungen werden exemplarisch aufgezeigt. Wenn das Forschungsfeld als „ethical enviroment“ (Guillemin \& Gillam 2004: 271) wahrgenommen wird, dann gewinnen ethische Dilemmata im Forschungsprozess auch erkenntnisförderliche Relevanz. ${ }^{13}$ Im Folgenden wird aufgezeigt, unter welchen Bedingungen und Schwierigkeiten der Forschungsprozess strukturiert war und mit welchen ethische Bedenken und Dilemmata sich Forscherinnen konfrontiert sahen.

\subsection{Gatekeeper*innen}

Gatekeeper*innen sind als Schlüsselpersonen und „Türöffner*innen“ bekannt, welche den Zugang ins Feld eröffnen oder die Erlaubnis über den Feldaufenthalt erteilen oder verweigern. Der Zugang zum Feld ist auf Seiten der Forscherin mit den Gatekeeper*innen auszuhandeln und herzustellen (Breidenstein et al. 2013: 52).

Im Forschungsprojekt lag die Besonderheit mehrerer Gatekeeper*innen vor. Um die Kinder und Familien zu erreichen, wurde vorher der Zugang über Heimleitung und teilweise Mitarbeiter*innen in allen Forschungstandems gelegt und das Einverständnis eingeholt, um in den Unterkünften für geflüchtete Menschen forschen zu dürfen. Um in Kontakt mit Eltern und Kindern in den Unterkünften treten zu können, waren zunächst die Einrichtungsleitungen von den Vorhaben zu überzeugen. Damit erhielten Einrichtungsleitungen eine machtvolle Position gegenüber Erziehungsberechtigten, die es ihnen erlaubte, den Kontakt, die Forschungsanfrage sowie die Informationsweiterleitung an Eltern und Kindern zu verhindern. Die Heimleitung sorgte für die Erlaubnis, die Unterkunft zu bestimmten Zeiten und Bedingungen betreten zu dürfen. Das räumliche Betreten der Unterkünfte wurde bereits am Eingang vom Sicherheitspersonal kontrolliert. Um die Familien zu erreichen, informierten Mitarbeiter*innen die Forscherinnen über Abläufe, Regelungen und Räumlich-

13 Die Eigenlogik des Feldes und sein dynamischer Kontext auf asylpolitischer, institutioneller und interaktionaler Ebene sind durch Veränderungen, Unsicherheiten und Ambivalenzen im Forschungsdesign gekennzeichnet; siehe den Beitrag von Wihstutz „Das Forschungsprojekt und sein Design“". 
keiten. Teilweise gab es Mitarbeiter*innen, die Vertrauenspersonen von Familien waren und die Forscherinnen bei den Familien vorstellten.

In den meisten Familien war das Arbeiten mit Sprachmittler*innen relevant, um Eltern und Kinder zu informieren und nach einer Forschungsteilnahme zu fragen, was Zeit im Aufbau einer Beziehung als auch häufig mehrere Besuche erforderte. Die Abhängigkeit der Kinder im Rahmen des generationalen Verhältnisses äußerte sich unter anderem darin, dass sie für ihre Beteiligung an einem Forschungsprojekt die Einwilligung ihrer Eltern als Erziehungsberechtigte benötigten. Die Forschung mit geflüchteten und begleiteten Kindern stand entsprechend vor der Herausforderung, mindestens zwei Gatekeeper*innen passieren zu müssen, bevor das Interesse der Kinder an einer Forschungsteilnahme ermittelt werden konnte. Der Weg eines Zugangs gestaltete sich langwierig und schwierig. Um an der Forschung teilzunehmen, war es wichtig, dass alle Beteiligten dem Vorhaben zustimmten. Dabei gab es ebenfalls Konstellationen, in denen Kinder teilnehmen wollten, aber ein Elternteil diese nicht zuließ. Die Kinder konnten dadurch nur begrenzt und abhängig von ihren Eltern entscheiden, ob sie teilnahmen. Dies stellte eine häufige forschungsethische generationale Diskrepanz dar (Alderson \& Morrow 2011: 107; Christensen \& Prout: 485; Phelan \& Kinsella: 86; Punch 2002: 323). Damit wird deutlich, dass rechtliche Vorgaben und Regulierungen nicht unbedingt mit ethisch relevanten Gesichtspunkt der Selbstbestimmung und Freiwilligkeit der Teilnahme von Kindern selbst (unabhängig von den Erziehungsberechtigten) als deckungsgleich wahrgenommen werden können.

\subsection{Informierte Einwilligung und Freiwilligkeit}

Die Frage nach der Einwilligung und Freiwilligkeit der in der Forschung involvierten Personen ist innerhalb des Kontextes aus unterschiedlichen Gründen besonders von ethischer Relevanz. Zum einen kann aus der Perspektive besonders junger Kinder hinterfragt werden, inwiefern sie das Ausmaß des Forschungsvorhabens verstehen und nachvollziehen können. Das gilt auch für Erwachsene und betrifft die angemessene Einschätzung darüber, was eine Teilnahme für sie selbst und für die Repräsentation ihrer Gruppe bedeuten kann. Gleichzeitig ist die Annahme, dass Kinder per se nicht verstehen, kein Grund, sie von Informationen zur Forschung auszuschließen - insbesondere wenn es sich um besonders junge Kinder handelt. Deutlich wird, dass die Kommunikation nicht nur generational, sondern auch national-sprachlich nicht eindeutig strukturiert ist. Das Einverständnis der jungen Akteur*innen im Feld ist vor allem als iterativer gegenseitiger Aushandlungsprozess zu verstehen (Guillemin \& Gillam 2004: 272; Mackenzieh et al. 2007: 307, 310). Das bedeutete ebenfalls eine gemeinsame Aushandlung darüber, unter welchen Bedingungen 
die Forschung stattfinden kann sowie den Austausch in verständlicher und zugänglicher Sprache zu führen. Angesichts von erschwerten Kommunikationsbedingungen war es Ziel, sowohl möglichst achtsam als auch aufmerksam gegenüber der Körpersprache der (jungen) Akteur*innen zu sein. Im Forschungsprojekt wurde der Zugang über eine schriftliche Erläuterung in einfacher und anschaulicher Sprache mit Bildern gewählt, welche die Einwilligung durch einen Handabdruck des Kindes symbolisch sicherstellte. Die Schwierigkeit bestand darin, die nötigsten Informationen für Kinder in einfacher Sprache zu erklären, wobei das Auslassen von möglicherweise wichtigen Informationen auch das Risiko in sich trug, nicht genug Transparenz zu schaffen.

Abb. 1: Version der Einverständniserklärung für Kinder in deutscher Sprache

Quelle: Eigene Darstellung

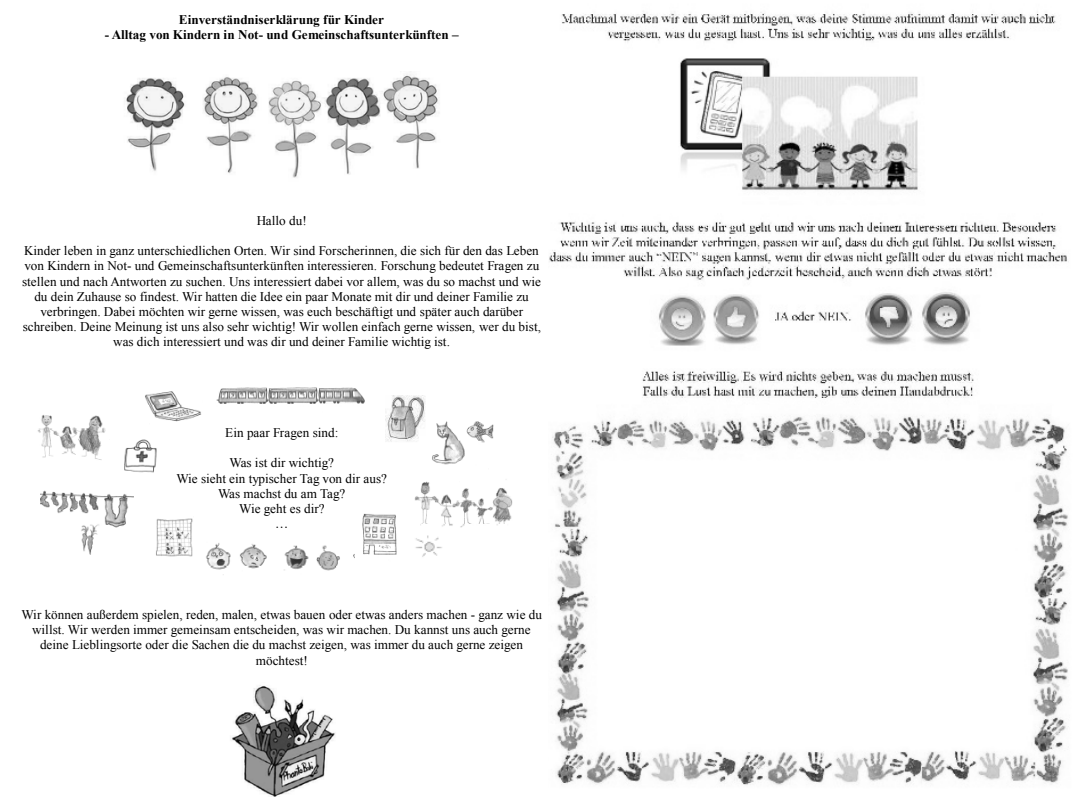

Neben der mündlichen Erklärung der Forschung wurden Informationsblätter über das Forschungsvorhaben in mehreren Sprachen an Eltern übergeben und kommuniziert, damit sich Familien für oder gegen eine Teilnahme am Forschungsprojekt entscheiden konnten. Im Verlauf des Forschungsprozesses machten die Forscherinnen den Kindern und Eltern wiederholt deutlich, dass sie ihre Beteiligung jederzeit beenden könnten. Nicht zuletzt waren die Forscherinnen bemüht, auch nonverbales Verhalten von Unwohlsein besonders junger Kinder zu erkennen und im Sinne von „ethischer Achtsamkeit“ (Phelan 
\& Kinsella 2013: 83) ernst zu nehmen. Im Sinne der Transparenz war durch die ethnografisch-explorative Herangehensweise eine Offenheit im Forschungsprozess vorgesehen, die zu Beginn der Forschung kommuniziert wurde. Es wurden vielseitige Erhebungsmethoden vorgestellt und mit den Familien und Mitarbeiter*innen der Unterkunft gemeinsam ausgehandelt. ${ }^{14}$

In Hinblick auf die gemeinsame Verständigung ist die Anerkennung und Wertschätzung der Sprache(n) der Familien zentral. Die Arbeit mit Sprachvermittler*innen war von grundlegender Bedeutung, für den Fall, dass die Forscherin die Sprache(n) der Familie nicht beherrschte. Im Projekt wurden Sprachmittler*innen gezielt, aber nicht bei jedem Feldaufenthalt aufgrund begrenzter finanzieller Ressourcen eingesetzt. Im Sinne der Transparenz war es bedeutsam, das Anliegen der Forschung, das Vorgehen und den gemeinsamen Forschungsprozess so zu gestalten, dass die Familien die Möglichkeit hatten, ihre Bedarfe auszudrücken. Außerdem war es wichtig Missverständnisse zu klären, da es durch die sprachliche Barriere Verständnisschwierigkeiten und verschiedene Erwartungen gab. Die klare Absprache mit den Sprachvermittler*innen erwies sich als notwendig und verlief nicht immer reibungsfrei. So gingen Inhalte in der Übersetzung verloren. Im Feld übernahmen die Dolmetscher*innen auch eigenständige Rollen, wie die der „Tante“, die eigenständig Fragen stellte und den Forschungsprozess mitgestaltete oder Süßigkeiten mitbrachte. Hier mussten Forscherinnen reagieren, falls der Erhebungsprozess zu sehr davon vereinnahmt wurde. Zum einen erwies sich das Sprechen der Sprache der Familie als wichtiger Vorteil, auch im Vertrauensaufbau und um überhaupt einen Zugang zur Familie zu erlangen. Gleichzeitig war es herausfordernd für die Forscherinnen, die Dolmetscher*innen auf ihre Funktion als Mittler*in hinzuweisen.

Durch die Forschung wurden Hoffnungen auf eine Verbesserung der rechtlichen Lage oder auch auf Unterstützung geweckt. Dies betraf insbesondere Familien mit unsicherer Bleibeperspektive. Die Erwartung an Forscher*innen, zur Verbesserung der Aufenthaltsperspektive beizutragen, ist keine Seltenheit (Krause 2016: 8). In Anbetracht dessen achteten die Forscherinnen darauf, keine unrealistischen Erwartungshaltungen zu wecken. Die Weitergabe von Halbwissen im Flucht- und Asylkontext kann unter Umständen schwerwiegende Folgen für betroffene und von Abschiebung gefährdete Familien mit sich führen. Forscherinnen gaben daher vielmehr Auskunft über Beratungsstellen, Dolmetscher*innen und Wissen über Verläufe von Asylverfahren. Sie verwiesen an dritte Personen, die über professionalisierte Expertisen verfügten. Falls ihre Unterstützungsmöglichkeiten begrenzt waren, wurde dies ebenfalls transparent kommuniziert, was beispielsweise psychosoziale Hilfeleistungen betraf, die verschiedenen institutionellen Zuständigkeiten unterlagen.

14 Diese Herangehensweise, die je Familie verschieden umgesetzt wurde, wird beispielsweise methodisch im Beitrag von Fichtner \& Trần angedeutet und beschrieben. 
Weitere Asymmetrien spiegelten sich in der Erreichbarkeit von Familien wider, welche nicht (lateinisch) alphabetisiert waren. Hier stellte die formale Einverständniserklärung selbst eine Barriere als bürokratisch formalisiertes Verfahren dar und wurde negativ antizipiert. So war es dieser formale Prozess zu Beginn der Erhebung, der eine Romafamilie unter anderem daran hinderte, an der Forschung teilzunehmen. Die Ethikkodizes fordern eine „, besondere Anstrengung" in der Forschung mit marginalisierten Menschen (beispielweise mit geringem Bildungsgrad) (von Unger 2014: 28). Häufig wird vor der Erhebung die Freiwilligkeit und das Einverständnis durch eine formelle Zustimmung sichergestellt. Die Frage, ob auch ein mündliches Einverständnis ausreicht, ist damit ebenfalls von Relevanz und kann im konkreten Kontext ausgehandelt werden. Eine weitere Möglichkeit besteht im Finden von Alternativen. Deutlich wird an dieser Stelle, dass formalisierte ethische Praktiken auch hinderlich für die Forschungsteilnahme sein können. Allgemein kann festgehalten werden, dass bereits der Prozess der Auswahl und des Zugangs der Forschungsteilnehmer*innen zur Forschung als selektiv betrachtet werden kann. Dieser Prozess kann damit auch systematisch Verzerrungen in der Stichprobe als auch Einseitigkeiten in den repräsentierten Perspektiven widerspiegeln.

\subsection{Anonymität und Vertraulichkeit}

Die personenbezogene Datenerhebung in den Unterkünften erlebten die Forscherinnen häufig als ambivalent durch fluide Grenzen zwischen öffentlichem und privatem Raum. Die Achtung und die Gewährleistung von Privatsphäre war nicht vollständig umsetzbar, da viele Räume kollektiv von Mitarbeiter*innen, Bewohner*innen, Familien und Forscher*innen genutzt werden mussten. Fehlende Rückzugsmöglichkeiten charakterisieren den institutionalisierten Raum der Unterkunft. Das verweist auf die spezifische Eigenlogik des Feldes in der die (als temporär angedachte) Unterbringung ${ }^{15}$ von geflüchteten Menschen, die sich räumlich unter den Augen einer gewissen und permanenten Öffentlichkeit widerspiegelt. Beispielsweise spielte sich eine Forschungssituation mit einer Familie ausschließlich in einem Kinderbetreuungsraum ab, um das Bewohner*innenzimmer als Privatsphäre der Familie zu achten. Das Forschen in den Unterkünften stand vor dem Dilemma, in die Privatsphäre der

15 Hier gibt es rechtliche Bezüge, welche die Unterbringungsdauer in verschiedenen Einrichtungen definieren. Diese wurden aber in den letzten Jahren mitunter ausgedehnt. Aus der Praxis haben uns Mitarbeiter*innen der Unterkünfte aufgezeigt, dass Erstaufnahmeeinrichtungen als Gemeinschaftsunterkünfte geltend gemacht werden. Viele Kinder und Familien haben in diesen Einrichtungen praktisch ihren zentralen Lebensmittelpunkt. Die Kinder sind teilweise in Deutschland geboren und die Unterbringungsverhältnisse stellen sich als konstitutiven Ort des Aufwachsens dar. 
Familien, die ohnehin räumlich häufig eingeschränkt war und von mehreren Menschen geteilt wurde, einzudringen. Selbst in die Flure zogen sich Kinder zurück oder spielten. Geschaffene Rückzugsräume von Kindern und Jugendlichen in öffentlicheren Räumen waren einsehbar und zugänglich. Mit einer Familie zu forschen, bedeutete auch Einblicke in das tägliche Leben weiterer Bewohner*innen zu bekommen. Dieser Umstand lag daran, dass Kochmöglichkeiten, Wasch - und Sanitäranalgen, Gemeinschaftsräume und Kinderbetreuungsräume gemeinsam genutzt wurden.

Als forschungsethisch herausfordernd erwies sich der Austausch mit Mitarbeiter*innen über Forschungserkenntnisse. In der Kommunikation mit Mitarbeiter*inne wurden die personenbezogenen Daten der Familien abstrahiert. Rückschlüsse auf konkrete Personen wurden durch Aussagen und Erfahrungen in allen Unterkünften verallgemeinert. Ein wesentliches Dilemma war es, wenn Mitarbeiter*innen im Kontakt mit Familien standen, welche an der Forschung teilnahmen. Die Anonymität der Familien gegenüber den Mitarbeiter*innen konnte damit nicht vollständig gewährleistet werden, da implizite Schlussfolgerungen von Mitarbeiter*innen auf Familien getroffen werden konnten. Auf Grund der räumlichen Struktur der Unterkünfte wurde deutlich mit wem geforscht wurde und mit wem nicht. Der Schutz und die Anonymität der Forschungsteilnehmer*innen war unterkunftsintern leicht aufzuschlüsseln. In öffentlicheren Räumen, wie im Hof, in den Fluren oder in Räumen der Kinderbetreuung, war ebenfalls für weitere Bewohner*innen ersichtlich, mit welchen Familien geforscht wurde.

Die Daten der Erhebung und die Ergebnisse der Forschung ließen teilweise Rückschlüsse auf Personen, Projekte und Orte zu und war für Insider*innen im Feld erkennbar. Daher wurden im Laufe der Pseudonymisierung von Unterkünften unterkunftsspezifische Eigenschaften verallgemeinernd umgeschrieben. Die Anonymität und Vertraulichkeit ist nicht nur gegenüber den Familien, sondern ebenfalls gegenüber den Einrichtungen, in denen geforscht wurde, einzuhalten. Das Ermessen, was auf Einrichtungen, Personen und Projekte zurückführbar ist und was nicht, ist im Genauen auszuhandeln. Dies erwies sich in der Praxis als schwierig, sodass eine Unterkunft ihr Einverständnis nachträglich zurücknahm und die Ergebnisse der Datenauswertung nicht veröffentlicht wurden.

\subsection{Schadensvermeidung und -abwägung}

Der forschungsethische Aspekt der Nichtschädigung ist gekennzeichnet durch das Abwägen von Risiken, die durch das Forschungshandeln im Feld entstehen können. Schäden sollen möglichst vermieden bzw. eingeschränkt werden. Die Reflexion über mögliche schädigende Folgen aus der Forschung bedarf beson- 
derer Aufmerksamkeit. Schäden sind nicht nur als physische Schäden zu begreifen, sondern beziehen soziale und emotionale Schäden, die kurz- und langfristig entstehen können, mit ein (Guillemin \& Gillam 2004: 272). Hugman et al. (2011) formulieren das Prinzip ,,do no harm“ is not enough“ um auszudrücken, dass Nichtschädigung als ethisches Prinzip mit marginalisierten Menschen nicht ausreicht und darüber hinaus gehen muss. Andersherum kann das Forschungsfeld entlang der Prämisse ,not ,do no harm ' is not enough“ als Prinzip der Nichtnichtschädigung formuliert werden. Die Forschung ist in einem machtvoll strukturierten Feld verstrickt und eingebettet. Sie ist von negativen und schädigenden Auswirkungen auf die Akteur*innen im Feld nicht ausgenommen.

Diese Perspektive eröffnet eine Sensibilität gegenüber negativen Folgen von Forschung als „harmful research“. In der Literatur zur Forschung mit geflüchteten Menschen wird konstatiert, dass ,gut gemeint" nicht gleichzusetzen sei mit ,gut gemacht“. ${ }^{16}$ So kann sich das Erzählen von schmerzlichen Erfahrungen gegenüber der Forscherin als schädigend auswirken, wenn nicht angemessen auf diese Erfahrungen eingegangen wird. Dabei ist ein sensibler Umgang mit den Erfahrungen und Fragen seitens der Forscherin und konkreter Abwägung bedeutsam. Beispielsweise wurde in Anwesenheit eines Kindes über Folter, Tod und Hafterfahrungen während der Flucht gesprochen. Zwischen Forscherinnen wurde dabei ausgehandelt, dem Erzählbedürfnis des Elternteils Aufmerksamkeit zu schenken und dem Kind die Möglichkeit gegeben, den Raum mit der Mutter zu verlassen, um der Erzählung somit nicht durchweg ausgesetzt zu sein. In der Literatur ist dokumentiert, dass geflüchtete Menschen sich in Publikationen nicht angemessen repräsentiert sehen oder sich als Informationsquellen reduziert fühlen. Sie äußerten, dass das aufgebaute Verhältnis zu Forscher*innen für Erhebungszwecke funktionalisiert und ausgenutzt worden sei (Hugman et al. 2011: 1277 ff.; Krause 2016: 10; Pittaway \& Bartolomei 2013: 156). Um solche Beschwerden überhaupt formulieren zu können, hilft es in einen Austausch darüber zu kommen. Dies kann im Forschungsprozess miteingeplant werden und kann unter Umständen interessante Impulse für den Auswertungsprozess liefern. Beispielsweise wurde am Ende der Erhebung die Wahrnehmung von Kindern auf den Forschungsprozess reflektiert und abgefragt. Forscherinnen wurden als ,Meerjungfrauen mit Zauberkräften“ beschrieben und eine Forschungsteilnahme als „sehr cool“ empfunden. Begründet wurde das mit der gemeinsam verbrachten Zeit und der Möglichkeit, dass Kinder entscheiden konnten, welche Aktivitäten sie mit Forscherinnen nachgehen wollten.

Dennoch gab es im Erhebungszeitraum auch negativ erlebte Erfahrungen von Kindern, die durch Konflikte entstanden sind. Die Forscherinnen konnten

16 Wohlgemeinte Forschung ist nicht davon frei, selbst Schäden zu verantworten, da die Gefahr besteht, diese nicht als ,lack of attention to related ethical aspects“ zu berücksichtigen (Hugman et al. 2011: 1277). 
nicht auf alle Interessen und Bedürfnisse gleichermaßen eingehen. Sie waren im Umgang mit starken Konflikten auch teilweise überfordert. In Anlehnung an die besondere Feldcharakteristik einer wahrgenommenen Ressourcenknappheit stellte die Praxis für die Forscherin eine Herausforderung dar. Dabei waren eingebrachte materielle (Stifte, Papier etc.), personelle und zeitliche Ressourcen begrenzt. Kindzentrierte Forschungsmethoden wie Malen, Basteln, Hausbegehungen und gemeinsames Spiel im Hof und die Aufmerksamkeit der Forscherin waren eine begehrte Ressource in der Unterkunft. Diese traf auf häufig erlebte Ressourcenknappheit von Familien und Kindern, welche sich durch das Einbringen materieller und immaterieller Anreize in die Forschung verstärkte. Sie gaben Anlass zu Neid, Besitzverhandlungen und Konflikten unter Kindern in der Kinderbetreuung. Auch die einschränkende Fokussierung der Forschungsfrage auf Kinder bis zu einem Alter von sechs Jahren bot Anlass für Konflikte unter Geschwistern, die in die Forschung, falls Interesse bestand, eingeschlossen waren. Die exklusive Nutzung von Räumen für forschungsteilnehmende Kinder und Forscherinnen führte dazu, dass ältere Kinder und Kinder, die nicht an der Forschung teilnahmen, den Raum nicht mehr für sich nutzen konnten. Da Räume für Spiel begrenzt und begehrt waren, führte dies zu Konflikten zwischen Kindern und Mitarbeiter*innen, welche den Raum für die Forschung exklusiv reserviert hatten. Diese Konflikte wurden in einem Fall auch von einer Mitarbeiterin an Eltern weitergetragen. Die Schäden oder Nachteile, die durch die Forschung entstanden, schlossen damit weitere Kinder der Unterkunft ein, die nicht explizit zum Sample gehörten. Die durch die Forschungspraxis verursachten Ungleichbehandlungen im Feld sind als Schadensverursachung durch die Forschung zu verstehen. Diese galt es möglichst zu vermeiden, was in der Praxis angesichts der Ressourcenknappheit schwierig realisierbar war. Hierbei stellt sich die Frage, wie sich die Forscherin zu Schäden verhalten sollte, die nicht durch sie verursacht waren oder als symptomatisch für ein umfassenderes Problem (z.B. Ressourcenknappheit durch prekäre Lebenslagen in Unterkünften) verstanden werden kann. Die Aushandlung zur Verantwortung, welche der Forscherin zukommen kann, um solche Schäden möglicherweise abzuwenden, bleibt schwierig zu beantworten. Zudem kann eine teilnehmende Beobachtung der Forschenden ohne explizite Intervention in der Rolle als Zuschauer*in unter Gesichtspunkten der Affirmation bestehender Machtverhältnisse problematisiert werden - beispielsweise in Hinblick auf Abschiebungen oder dem Beiwohnen von diskriminierenden Praktiken, von Konflikten unter Kindern oder dem Erleben von respektlosen und paternalistischen Verhalten von Mitarbeiter*innen gegenüber Eltern. Es entstand Verunsicherung dahingehend, das eigene Tun oder Unterlassen situationsangemessen abzuwägen.

„When a human being is in need and the researcher is in a position to respond to that need, non-intervention in the name of ,objective" research is unethical" (Mackenzieh et al. 2017: 316). 
Dieses Zitat konstatiert, dass es ein Nichtverhalten auf kommunikativer und handelnder Basis für die Forscherin im Feld nicht gibt. Das Verständnis von „Objektivität“ wird mit ausbleibender Responsivität als Nichtintervention kritisiert. Diese normative Position als Interventionsanlass stellt die empathische Anteilnahme mit Mitmenschen in den Vordergrund. Diese (emotionale) Anteilnahme kann methodologisch, epistemologisch und analytisch-reflexiv als gewinnbringend identifiziert werden. ${ }^{17}$ Dabei kann Objektivität auch als Unabhängigkeit der Beobachterin verstanden werden. Diese Lesart impliziert eine absolute und objektivierte epistemologische Annahme und kann auch als „Selbsttäuschung“ der erkenntnisproduzierenden Forscherin gedeutet werden. Die Annahme entkoppelt die Wissensproduktion von ihrer zugrundeliegenden Methodik: Das Prinzip einer „wertfreien“ oder „neutralen“ Forschung deutet auf die Gefahr der Verkennung der eigenen Position und der Interaktivität sowie Interventionshaftigkeit der Forschenden im Forschungskontext hin (Breuer 2004: Abs. 18).

Ein wichtiger Ansatzpunkt in der Auseinandersetzung mit dem Handeln von Forschenden sind erlebte Irritationsmomente im Feld, die auch als „moment-to-moment ethical choices“ (Kaukko et al. 2017: 16) bezeichnet werden. Im Verlauf des Forschungsprozesses wurden Forscherinnen mit dem Bescheid zur Abschiebung einer Familie konfrontiert. Die Forscherinnen identifizierten diese emotional aufgeladene Situation als ethisch bedeutsam. Hier war das Ausbalancieren von emotionaler Nähe und Distanz zentral. Die Forscherinnen konnten sich dem Vorgang nicht entziehen. Vielmehr waren sie gefordert, sich in irgendeiner Form zu verhalten, was sich in Parteilichkeit für die Familie und ihr Bleiberecht äußerte. Solche Fragen im Mikrokontext und die Entwicklung von Standpunkten und Haltungen als Forschende sind situationsspezifisch und kontextgebunden reflektierbar. Diese Situation der Abschiebung verdeutlichte den Forscherinnen, dass Mitgefühl und Solidarisierungsprozesse auch Teil einer wissenschaftlichen Praxis sein können. Unabhängig von den Bewertungsprozessen der jeweiligen Person geht es darum wertebezogene und normative Annahmen und Handlungen als aktiven Prozess der Erkenntnisproduktion offen zu legen. Eine weitere und vertiefende Auseinandersetzung mit solchen Spannungsverhältnissen und eigener Emotionalität kann auch ethische Relevanz verdeutlichen und als handlungsleitend in die Forschungspraxis zurückführen (Guillemin \& Gillam 2004: 261 f.).

Die Frage, was nun als legitim und illegitim, ethisch und unethisch oder auch angemessen und unangemessen im Sinne ,guter" Forschung diskutiert wird, obliegt unter anderem der (diskursiven) Aushandlung der Forschungsgemeinschaften und ist selbst Gegenstand normativer Setzungen und Deutungshoheiten innerhalb der Wissenschaftscommunity. Dass Forschung auch Schaden verursachen und Gewaltverhältnisse reproduzieren kann, wirft die Frage

17 Hierzu entlang konkreter empirischer Beispiele von Fichtner \& Trần (2018) aufgegriffen. 
auf, inwiefern Forschung selbst legitimationsbedürftig ist in Hinblick auf die Einhaltung ethischer Richtlinien. Diese Richtlinien sind weitaus mehr als nur eine abzuarbeitende Formalität. Die Institutionalisierung von ethischen Richtlinien ist dabei genauso bedeutsam, wie das Bewusstsein und die Selbstverpflichtung der Forscher*innen zum Wohl der beteiligten Akteur*innen und der Gesellschaft beizutragen. Aus diesen Erörterungen lässt sich die Bedeutung einer unabhängigen Institution ableiten. Diese Stelle könnte für Forschungsteilnehmer*innen etabliert werden, um ihren Fragen und Beschwerden Raum zu verschaffen und ihre Rechte und Position im Forschungsprozess zu stärken. Diese könnte darüber hinaus auch als Beratungsinstanz für Forscher*innen genutzt und betrieben werden.

\subsection{Strukturelle Gewalt und Forschungsbeziehung}

Ethische Fragen können in Anlehnung an Persönlichkeits- und Menschenrechte unter asylpolitischen Diskursen viel grundlegender gestellt werden. Das politisch gewaltvoll erlebte Feld (Akesson 2014:4) im Asyl- und Fluchtkontext ist auch hinsichtlich des im Grundgesetz verbrieften Artikels 1 zur Achtung der Menschenwürde kritisch zu hinterfragen, da die persönliche Integrität, Anerkennung und Selbstachtung von Menschen nicht in allen wesentlichen Bezügen respektiert wird (Brumlik 2017: 25). Die politische Identität als Kind und als geflüchtetes Kind ist mit gravierenden rechtlich regulierten Einschränkungen verbunden. Diese können als strukturelle Gewalt bezeichnet werden ${ }^{18}$. Es ist der asylrechtlichen Praxis der Zuteilung der Unterkunft als Aufenthaltsort geschuldet, dass das Zusammenleben in einer von Kindern erlebten ,reglementierten Zwangsgemeinschaft" stattfindet und dass Familien in beengten Verhältnissen leben müssen, in denen häufig unhygienische Sanitärräume geteilt werden, mit der Konsequenz, dass diese Lebensbedingungen als nicht kindgerecht eingestuft werden (World Vision \& Hoffnungsträger Stiftung 2016: 49). Die strukturellen Bedingungen des würdevollen Aufwachsens von jungen Menschen und Prinzipien der allgemeinen Gleichbehandlung und Selbstbestimmung können daher grundsätzlich in Frage gestellt werden, wenn Menschen auf Grund ihrer Migrationsbewegung kriminalisiert, illegalisiert oder gewaltvoll abgeschoben werden. Zu flüchten bedeutet ein hohes Maß an Unsicherheit, mangelnden Schutz und immense Abhängigkeiten von asylpolitischen Diskursen, institutionellen Willkürlichkeiten und Gesetzesänderungen.

Menschenrechte wie Bewegungsfreiheit, Selbstbestimmung und das im Artikel 3 der UNKRK beste Handeln im Interesse des Kindes treten angesichts dominierender nationaler Gesetzgebung und Asylpolitik in den Hintergrund.

Zu Gewaltverhältnissen in Unterkünften wurde der Beitrag von Schulz-Algie verfasst. 
Beispielsweise werden weitere sichere Herkunftsländer konstatiert, Verantwortungen exterritorialisiert (beispielsweise durch das Dublin-Verfahren, den sogenannten Türkei-Deal und die Aufstockung des außereuropäischen Grenzschutz), Abschiebungen werden beschleunigt und Ankerzentren eingerichtet ${ }^{19}$. Der Widerspruch zwischen einem an den Kinderrechten orientierten forschungsethischen Anspruch und den entmutigenden Realitäten asylpolitischer Diskurse und Politiken stellt ein juristisches Spannungsverhältnis und ethisches Dilemma dar. Dabei lähmen unsichere Bleibeperspektiven die Teilhabemöglichkeiten von Familien und Kindern und schränken ihr Wohlbefinden ein. Kinder äußerten in der Forschung den Wunsch nach einem eigenen Haus. Die Bewusstwerdung über reale Zustände vor Ort und noch restriktiverer Asylpolitik machen die Grenzen der eigenen Handlungen und Wirkmächtigkeit von Forschungen deutlich. „Gute“ Forschung in ,schlechten“ Verhältnissen zu betreiben ist mit Widersprüchlichkeiten verbunden.

Bestrebungen der Forscherinnen, die Bedingungen in den Sammelunterkünften zu verbessern, führen auch dazu, eben diese Strukturen zu erhalten. Die Sammelunterkünfte selbst sind in Hinblick auf das Kindeswohl hinterfragbar und markieren den temporären Ausnahmezustand, der zur Regel geworden ist. Kinder wurden dort geboren: Sie lernen und verinnerlichen die Verhältnisse in denen sie aufwachsen. Sie haben ein subtiles Gefühl dafür nicht zugehörig genug zu sein, um als vollwertige Gesellschaftsmitglieder anerkannt zu werden. Ihre Erfahrungen sind von Diskriminierung und Einschränkungen geprägt, die einen selbstverständlichen Teil ihrer Lebensrealität ausmachen. Durch die Betonung der Akteur*innenschaft von Kindern wird intendiert ihre Lebensrealitäten und Kindheiten wertzuschätzen und anzuerkennen, ohne die ungleichen Bedingungen des Aufwachsens in Unterkünften aus dem Blick zu verlieren. Auch die Forschung steht im Spannungsverhältnis, Bedingungen in den Unterkünften zu analysieren und kritisieren, um damit zu Verbesserung der Lebensbedingungen junger Kinder vor Ort beizutragen. Das Forscherinnenteam plädiert dafür, dieses geschaffene Sondersystem als lagerähnliche Unterbringungsform abzuschaffen. Gleichzeitig ist die Forderung realpolitisch hinterfragbar und der Übergang in Regelstrukturen nicht ohne Weiteres zu realisieren.

Aufgrund von asylrechtlichen Normierungen kam es im Feld zu plötzlichen Beziehungsabbrüchen. Familien wurden abgeschoben oder tauchten unter. Die asylrechtliche Praxis von Abschiebungen ist ethisch und rechtlich hinterfragbar. Sie greift in das Wohl, die Selbstbestimmung und die Bewegungsfreiheit der Familien massiv ein. Beziehungsaufbau und -abbruch sind forschungsethisch relevante Fragen und stellen nur die Spitze des Eisbergs angesichts des asylrechtlichen Systems dar.

19 Eine differenziertere Betrachtung liefert der Beitrag von Wihstutz „Mittendrin und außen vor". 
Die Forscherinnen hatten nur bedingt Einfluss auf die Beziehungsgestaltung. Teilweise war es nicht klar, ob die teilnehmenden Familien beim nächsten Feldbesuch noch in der Unterkunft waren. Die Forschungsbeziehung war dadurch von Unsicherheit geprägt und Forscherinnen konnten nur bedingt Einfluss auf die Gestaltung nehmen:

„Forschungsbeziehungen sind fragile Gebilde. Die Beteiligten kommen eher zufällig zusammen, sie verbindet nur eine kurze Geschichte, eine Zukunft ist nicht absehbar. Sie beginnen einen komplexen Kooperationsprozess, für den es kaum Routinen gibt. Beide Seiten müssen sich aufeinander einlassen, ohne rechte Gründe und Sicherheiten für Vertrauen zu haben" (Wolff zitiert nach Breidenstein et al. 2013: 62).

Der Beziehungsaufbau der Forscherinnen zu geflüchteten Familien und insbesondere Kindern hat im Verlaufe der Forschung eine eigene Dynamik gewonnen. Zum einen betrifft das den Zugang zu den Familien, welcher tendenziell durch fluchtspezifische Erfahrungen, unter anderem auch durch Misstrauen, geprägt war. Diese Beziehungen waren ,fragile Gebilde“ und oblagen auf Seiten der Forscherinnen einer besonderen Verantwortung, insbesondere bei mehrmonatigen Feldaufenthalten, wie es im Projekt der Fall war. Weiterhin wurden die Beziehungen zu den Kindern häufig positiv als Ressource und der Besuch in der Unterkunft teilweise als „Highlight“ im Alltag der Kinder erlebt. Ein Beziehungsaufbau zu den Kindern war gewollt und auch interessengeleitet. Die Entscheidung, wie nach Abschluss der Feldarbeit damit weiter verfahren werden sollte, lag in der Verantwortung der einzelnen Forscherinnen. Die emotionale Nähe und Distanz in der Forschungsbeziehung verschwimmt im Spannungsfeld einer authentischen als auch funktionalisierten Verbindung von Menschen. Sie wird auf Grund der Forschung eingegangen und ist Produkt einer besonderen Art von Beziehung, welche die „Arbeit der Ethnografen einfacher oder komplizierter" (Breidenstein et al. 2013: 62) macht. Beziehungen haben auch über den Forschungskontext für Familien und Kinder als auch für die Forscherinnen an Bedeutung gewonnen. Manche Beziehungen zu Kindern wurden daher nach Projektende aufrechterhalten, da die Kinder sich an die Forscherinnen durch regelmäßige Besuche gewöhnt hatten und ihnen und ihren Familien die Aufrechterhaltung des Kontakts wichtig geworden war beziehungsweise dieser eingefordert wurde. Teilweise wurden Besuche seltener und es gab ebenfalls Fälle, wo das Forschungsende bedauert aber hingenommen wurde oder durch asylrechtliche Bestimmungen abgebrochen wurde. Zum Teil wurde der Kontakt online über soziale Netzwerke gehalten. 


\section{Rolle der Forscherin im Feld}

Angesichts forschungsethischer Debatten gibt es den Standpunkt, die Interessen der in der Forschung beteiligten Akteur*innen aus ethischen Gesichtspunkten möglichst über die Interessen der Forscher*innen selbst zu stellen (Hugman et al. 2011: 1275). Als Bestandteil der Erhebungsinteressen sind in der Forschung mit geflüchteten Menschen die Bedarfe der Akteur*innen im Feld nicht zu vernachlässigen, sondern ihnen Rechnung zu tragen (Krause 2016: 10). Die Bedeutung der Reflexion um die Rolle der Forscher*innen im Feld (Hugman et al. 2011: 1278) macht deutlich, dass diese Rollen selbst Aushandlungsgegenstand der Forschung sind. Damit verbundene eingenommene multiple Rollen im Feld werden damit sichtbar gemacht. Bei genauer Betrachtung gibt es nicht die eine Forschungsrolle als Forscher*in. Angesichts vielseitiger Interessenlagen gestaltet sich dieser Prozess der Aushandlung der eigenen Rolle als anforderungsreich (Christensen \& Prout 2002: 488; Norstedt \& Breimo 2016: 2; Stodulka 2014: 186). Die Herausforderung besteht, zwischen Selbst- und Fremdpositionierungen $\mathrm{zu}$ unterscheiden und Forschungsinteresse, Handlungserfordernisse und Bedarfe im Feld abzuwägen.

Im Forschungsprojekt agierten in einem institutionellen Setting unterschiedliche Akteur*innen (Kinder, Familien und verschiedenen Mitarbeiter*innen). Exemplarisch wurde dies deutlich, als Heimleitungen sich von den Forscherinnen eine Außenperspektive, die Auseinandersetzung mit schwierigen oder belasteten Familien oder auch die Unterstützung von besonders benachteiligten Familien wünschten. In der Kinderbetreuung wurden Forscherinnen mitunter als verantwortliche Aufsichtspersonen adressiert. Von Kindern wurden Forscherinnen als erwachsene Streitschlichterinnen eingesetzt. Eltern adressierten die Forscherinnen unter anderem als Ratgeberinnen in Erziehungsfragen, beim Lesen von bürokratischen Dokumenten, bei der Vermittlung von therapeutischer Unterstützung oder mit dem Anliegen, ihr Kind besser verstehen zu können. Für Kinder hingegen waren die Themen Spiele spielen, eigene Ideen einbringen und realisieren als auch ungeteilte Aufmerksamkeit von Forscherinnen zentral.

Die Rolle der Forscherin ist damit nicht fix, sondern fluide. Sie wird von außen mitbestimmt und von der Forscherin mehr oder weniger entsprochen. Die Forscherin ist multifunktional und wird im Kontext unterschiedlicher Vereinnahmungsversuche oder Adressierungen sowie in Selbstpositionierungen verhandelt. Die Ausübung unterschiedlicher, wechselnder Rollen erlebten die Forscherinnen teilweise auch als Rollenkonfusion. Dies war Ausdruck vielseitiger Interessen, Bedarfe und Bedürfnisse verschiedener Akteur*innen im Feld sowie Charakteristikum des Feldes selbst, welches auch von Mitarbeiter*innen wahrgenommen wurde. Diese Momente haben auch erkenntnisförderliche 
Relevanz, um wiederum Aussagen über das beforschte Feld zu generieren. ${ }^{20}$ Teilweise erfuhren die Forscherinnen auch Loyalitätskonflikte. Beispielsweise wurden die Forscherinnen gebeten, bestimmte Themen (z. B. fehlender Internetzugang) oder bestimmte Informationen an die Heimleitung weiterzutragen. Bezüglich der gesundheitlichen Verfassung und therapeutischen Unterstützung von Kindern, war es schwierig für die Forscherinnen den Interessen der Eltern und den Interessen der Mitarbeiter*innen nachzukommen. Auch die Interessen der Kinder waren nicht in allen Fällen mit den elterlichen Interessen gleichzusetzen. Teilweise wurden auch sensible Informationen zum Kind in Erfahrung gebracht, um eine Handlungsbasis für eine gesundheitliche Unterstützung zu finden. Die Aushandlung der eigenen Entscheidungen im Forschungsfeld gegenüber einzelnen Akteur*innen war situationsbezogen auszubalancieren, je nachdem um wen und welche Aktivität es sich handelte. Dabei wurde die Absprache im Tandem als hilfreich empfunden. Die transparente Kommunikation über die Rolle der Forscherin wurde bedeutsam, um gegenseitige Erwartungen zu klären. Abgrenzungen der eigenen Rolle im Feld sind damit ebenso wichtig geworden wie die nötige Flexibilität und das Einlassen auf die Interessenlagen und Handlungserfordernisse aus dem Feld. Sprachbarrieren, Loyalitätskonflikte, fehlendes konkretes Kontextwissen oder einseitig verfügbare Informationen erschwerten dieses Anliegen.

In Verbindung mit der Rolle im Feld und der Frage nach gezielter Intervention und dem Verhalten der Forscherin sind Fragen praktischer Ethik von Bedeutung. Das Ad-hoc-Handeln im Feld betraf beispielsweise eine Situation in der die Forscherinnen das Verhalten einer technischen Aushilfskraft zu Kindern als unangenehm und grenzüberschreitend wahrnahmen. ${ }^{21}$ Solche ethischen Momente der Verunsicherung verdeutlichen Rollenkonflikte der Forscherinnen im Feld. Sie zeigen die Verunsicherungen und Ambivalenzen im Abwägungsprozess über die Entscheidung der Forscherin zu handeln oder Handlungen zu unterlassen. Hier wird deutlich, dass Fragen praktischer und prozeduraler Ethik mitunter verquickt und nicht ganz trennscharf sind. Richtlinien und ethische Grundsätze ausgewiesener Kodizes (,,procedural ethics“) sind hier nur bedingt übertragbar. Die Komplexität und Kontextspezifik von Forschung entfalten sich im Feld der praktischen Ethik (Guillemin \& Gillam 2004: 273).

Die Bewusstmachung über mögliche Konsequenzen durch die Reaktion der Forscherin, ihrer Befindlichkeit und Handlungsoptionen sind wichtige Bestandteile der Forschungspraxis. Potenzial in der Auseinandersetzung bieten aus ethischer Perspektive vor allem belastende, schwierige oder irritierende

20 Die konkrete Auseinandersetzung und Aufarbeitung mit diesen ethisch-bedeutsamen Momenten im Feld in Verbindung mit der Eigenlogik des Feldes als Kontextrelevanz mit Erkenntnismehrwert sind ausführlicher von Fichtner \& Trần (2018) beschrieben worden.

21 Weitere Ausführung zu einer konkreten Situation im Forschungsfeld vgl. Trần (2017). 
Erfahrungen im Forschungsprozess. Die Offenlegung emotionaler Involviertheit macht den Umgang mit eigener Emotionalität, Wahrnehmungen und Handlungen bearbeitbar. Dieser separate Raum für Forscher*innen bietet neben Psychohygiene und Austausch einen geschützten Rahmen der Auseinandersetzung. Dieser ermöglicht eine Distanz zu sich selbst zu entwickeln, Perspektiven zu überdenken und eigene Handlungsoptionen zu erweitern oder sich des eigenen Handelns bewusst zu werden. Darüber hinaus können Emotionen die Wahrnehmung und Interpretation der Daten verdichten. Emotionen selbst sind kein Hindernis in der Forschung, sondern haben einen epistemologischen Wert für eine empirisch-gestützte Selbstreflexivität (Stodulka 2014: 182 f.).

\section{Engagierte Forschung}

Die verschiedenen Grenzen und Schwierigkeiten forschungsethischen Handelns wurden exemplarisch durch Dilemmata und Anforderungen an den Forscherinnen aufgezeigt. Die Auseinandersetzung mit ethischen Fragestellungen trägt zur Bewusstwerdung der Verstrickung von Forschung in Machtverhältnissen, zum Beispiel durch die Krise der Repräsentation, aber auch zur Sensibilität und Reflexivität im Forschungshandeln bei. Deutlich wurde auch, dass es Dynamiken im Feld gibt, auf die die Forscherin wenig Einfluss nehmen kann - und dennoch ist sie gefordert, sich im Feld dazu zu positionieren und ihre Rolle zu finden und auszuhandeln.

Innerhalb dieser vielseitigen Verflechtungen gibt es trotz der Schwierigkeiten Mehrwerte, die am Ende dieses Beitrags erläutert werden. Wie bereits erwähnt, reicht das Prinzip der Nichtschädigung im Kontext der Forschung mit marginalisierten Personengruppen nicht aus. Die Forschung soll einen expliziten Nutzen für die Forschungsteilnehmer*innen haben (Mackenzie et al. 2007: 301) und kann in dieser Hinsicht auch zu sozialer Gerechtigkeit beitragen (Alderson \& Morrow 2011: 20; Hugman et al. 2011: 1275). Damit gibt es Überschneidungen zur partizipativen Forschung als „wertbasiertes Unterfangen“ mit dem Anliegen, auf soziale Gerechtigkeit hinzuwirken (von Unger 2014: 2).

„Ultimately, there are power differentials in the relationship that place significant responsibilities on the part of the professionals. [...] ,Do no harm ' is a necessary but insufficiant principle alone in such work. It needs to be integrated with respect, beneficence and justice in a more relational approach that can be auged in terms of what is offered back to participants in ways that are meaningful to them" (Hugman et al. 2011: 1276, 1284). 
Das Prinzip der Nichtschädigung ist unzureichend, wenn Forschung mit benachteiligten Personengruppen betrieben wird. Die Erweiterung der Verantwortung der Forscher*in bedeutsame Mehrwerte für Akteur*innen des Feldes herzustellen und mit dem Anliegen zu sozialer Gerechtigkeit beizutragen ist grundlegend. Die in der Forschung angestrebten Mehrwerte durch die Forscherinnen sind im Feld auf Ebene der Kinder beispielsweise die Etablierung von Möglichkeiten zur erweiterten Raumnutzung (z. B. „Legoraum“, Kinderbetreuungsraum), die sie mit den Mitarbeiter*innen zugunsten der Kinder vereinbaren konnten. Die Kinder wurden auch unterstützt, indem recherchiert wurde, ob bestimmt Hobbys oder Interessen in der Nähe realisiert werden können. Mitunter bekamen die Kinder Urkunden, Forschungsordner und Fotografien, die im Forschungsprozess entstanden, als symbolischer Akt der Anerkennung und als Erinnerung an die gemeinsame Zeit. Die Zeit, in der sich die Forscherinnen mit den Kindern beschäftigten, konnten die Eltern teilweise für sich nutzen, zum Beispiel für Behördentermine, zum Kochen, Schlafen, Aufräumen oder zur Erholung. Auch bei bürokratischen und juristischen Anliegen, in Fragen der Erziehung, Wohnungssuche oder bei der Therapieplatzfindung konnten die Forscherinnen als Informationsvermittlerinnen die Eltern unterstützen. Durch die Begleitung der Kinder und Familien wurden auch Erkenntnisse und auftauchende Bedarfe und Wünsche der Familien in einem Gespräch mit Heimleitung und Team angesprochen - beispielsweise in der Förderung der Herkunftssprache und dem Etablieren von Beteiligungs- und Beschwerdemöglichkeiten der Kinder. Durch den Feldaufenthalt konnte sensibel auf Kinderschutzrisiken in der Einrichtung aufmerksam gemacht werden ${ }^{22}$. Forscherinnen gaben den Familien und Mitarbeiter*innen der Unterkünfte Rückmeldungen über die Erhebung und Auswertung und weiteren Planungen im Projekt. Auch Rückmeldungen vielseitiger Art über die Erhebung und Auswertung wurden mit Familien und Mitarbeiter*innen der Einrichtung diskutiert.

Das Ansprechen von kinderrechtlichen Verstößen und die Betonung von Menschenrechten und des Bleiberechts für alle gehörten zur ethischen Verantwortung von Forscherinnen, die auch in der Öffentlichkeit wahrgenommen wurde. Die Forscherinnen betonen die Rechtsposition von Kindern als Rechtssubjekte. Es handelte sich um ein advokatorisches Projekt, in dem die Diskrepanz von Kinderrechten und asylpolitischen Regelungen herausgearbeitet wurde. Die Ergebnisse der Forschung wurden an die Familien und an die Mitarbeiter*innen in den Unterkünften zurückgemeldet sowie auf Tagungen der Fachöffentlichkeit vorgestellt. Alle an der Forschung Beteiligten wurden zur Abschlusstagung eingeladen; ein Angebot, das von den geflüchteten Kindern und ihren Eltern nicht angenommen wurde. Das wirft zusätzlich Fragen nach dem Format der Rückmeldung und der Zugänglichkeit der Forschungserge-

22 Siehe den Beitrag von Schulz-Algie. Durch ihr Zutun konnte die Einrichtung in der Vernetzung zu Institutionen mit ähnlichen Anliegen unterstützt werden, sowie das Thema Kinderschutz in Unterkünften bewusstere Auseinandersetzung finden. 
bnisse auf. In Anerkennung der Interessen von Kindern an (Bilder-)Büchern entstand im Verlauf des Forschungsprozesses die Idee, für die Kinder in den Unterkünften ein Kinderbuch in mehreren Sprachen zu entwickeln, wobei die Kinder in die Gestaltung aktiv einbezogen wurden. In diesem Buch sollten die Ergebnisse und Erfahrungen der Kinder und Familien kreativ und realistisch aufbereitet werden. Im Anschluss an die Feldarbeit wurde mit der Umsetzung dieser Idee als ein eigenes kollaboratives Projekt mit Kindern aus verschiedenen Unterkünften begonnen. Darüber hinaus entwickelte das Projektteam ein politisches Positionspapier und nahm öffentlich Stellung ${ }^{23}$. Der Transfer der Erkenntnisse in die Praxis wurde über Handlungsempfehlungen und Fortbildungen weiterverfolgt. Die Bereitstellung der generierten Erkenntnisse als Praxistransfers für Politik, NGOs, verschiedene Institutionen als Grundlage für Weiterbildungen in pädagogische Praxis und Austausch verfolgte das Ziel der Vernetzung auch mit Selbstorganisationen von Geflüchteten. Mit weiteren Kindern aus Sammelunterkünften wurde die Auseinandersetzung mit ihrem Alltag in Unterkünften weiterverfolgt. Die Ergebnisse wurden beispielsweise auf einem Kinderbuchfestival mit Forderungen und Wünschen der dort lebenden Kinder vorgetragen.

„,[R] esearchers do not enter the field just to ,hear' the children's stories for the sake of research. It means that researchers, who have been invited into the lives of refugee children, get up from the couch' and engage. [...] Practically, researchers should consider how they can truly add value to the lives of refugee children instead of disrespecting children by treating them as sources of data" (Kaukko et al. 2017: 20).

Damit kann Forschungspraxis auch als verändernde und engagierte Praxis begründet werden. Der unmittelbare Nutzen bzw. Mehrwert für die Forschungsteilnehmer*innen wurde in diesem Beitrag kritisch und in seinen Grenzen deutlich und in Dilemmata, Spannungsverhältnissen oder auch als Rollenkonfusion von Forscherinnen beschrieben. Dabei wurde das Forschungshandeln zum Wohle der geflüchteten Kinder und Familien als engagiertes Forschen exemplarisch hervorgehoben.

\section{Ausblick}

In diesem Beitrag ist der Versuch unternommen worden forschungsethische Überlegungen auf der Grundlage der eigenen Forschungserfahrung mit ge-

23 Die inhaltliche Ausführung des Positionspapiers im Beitrag von Wihstutz ,Zentrale Erkenntnisse und Empfehlungen“. 
flüchteten jungen Kindern im multidisziplinären Team in ihrer Vielschichtigkeit aufzuzeigen. Die Überlegungen beziehen sich primär auf die erlebte Forschungspraxis. Forschungsethische Fragen wurden im Zusammenspiel von Unsicherheiten, Irritationen und Ambivalenzen diskutiert. Die Notwendigkeit, die Praxis von empirischen Forschungen unter ethischen Gesichtspunkten darzustellen, wird bereits gefordert (von Unger et al. 2016: Abs. 19). Angefangen bei der reflektierten Subjektivität und der Reflexion des Forschungshandelns ist die Auseinandersetzung mit forschungsethischen Dilemmata bedeutsam und eine Bereicherung sowie Anlass für weitere Diskussionen in Forschung und Wissenschaft. Durch die Zielgruppe junger Kinder in Unterkünften für geflüchtete Menschen wurden konkrete forschungsethische Herausforderungen in diesem Feld der Reflexion und Analyse zugänglich gemacht. Das Forschen mit Kindern und allgemein mit geflüchteten Menschen braucht unter ethischen Gesichtspunkten besondere Aufmerksamkeit und tiefere Auseinandersetzung. Forschungsethisch verantwortungsvolles Handeln ist als dynamischer Prozess gemeinsamer Aushandlung zu verstehen. Dieser Prozess entsteht in Interaktion und wechselseitiger Bezugnahme und Empathie. Neben fixen entwickelten Standards, die ein wesentlicher Bestandteil von Forschungsethik sind, werden ethische Fragen erst im Kontext relevant sowie identifizier- und bearbeitbar.

Ziel dieses Beitrags war es, anhand der Reflexion der empirischen Forschungspraxis die Bedeutung ethischer Fragen und Fallstricke aufzuzeigen, die als integrale Bestandteile von Forschung eine lohnenswerte Perspektive bieten. Dabei sind Debatten über Forschungsethik nicht nur im akademischen Milieu zu führen. Es ist auch die Perspektive betroffener Menschen, mit denen und über die geforscht wird, einzubeziehen und mitzudenken. Gerade weil Machtverhältnisse kein Verhältnis auf Augenhöhe zulassen, wirft gerade diese Perspektive die Frage nach der Verantwortung der Forschenden auf. Die Auseinandersetzung mit ethischen Fragestellungen liefert Impulse für Haltung und Praxis in Forschung und Wissenschaft, deren Stellenwert aufgewertet und priorisiert gehört.

\section{Literatur}

Akkesson, Bree (2014): Geographies of Palestinian Children and Families: A Critical Review of the Research and a Future Research Agenda. In: Harker, Christopher, Hörschemann, Skelton, Tracey (Hrsg.): Conflict, Violence and Peace, Geographies Of Children and Young People 11. Singapore: Springer Science + Business Media, S. 1-18. 
Alderson, Priscilla \& Morrow, Virginia (2011): The Ethics of Research with Children and Young People - A Practical Handbook. London, California, Dew Delhi, Singapore: Sage.

Appadurai, Arjun (2006): The right to research. In: Globalisation, Societies and Education 4 (2), S. 167-177.

Bessell, Sharon, Beazley, Harriot \& Waterson, Roxana (2017): The Methodology and Ethics of Tights-Based Research with Children. In: Intervernizzi, Antonella, Liebel, Manfred, Milne, Brian \& Budde, Rebecca (Hrsg.): ,Children Out of Place' and Human Rights. In Memory of Judith Ennew. Schweiz: Springer Nature, S. 211-232.

Block, Karen, Warr, Deborah, Gibbs, Lisa \& Riggs, Elisha (2013): Addressing Ethical and Methodological Challenges in Research with Refugee-background Young People: Reflections from the Field. In: Journal of Refugee Studies 26 (1), S. 69-87.

Breidenstein, Georg, Kalthoff, Herbert, Hirschauer, Stefan \& Nieswand, Boris (2013): Ethnografie - Die Praxis der Feldforschung. Konstanz, München: UVK.

Breuer, Franz (2003): Subjekthaftigkeit der sozial-/wissenschaftlichen Erkenntnistätigkeit und ihre Reflexion: Epistemologische Fenster, methodische Umsetzungen. In: Forum Qualitative Sozialforschung 4 (2), http://www.qualitativeresearch.net/index.php/fqs/article/view/698/1509 (Zugriff 6.6.2017).

Brumlik, Micha (2017): Advokatorische Ethik. Zur Legitimation pädagogischer Eingriffe, 3. Auflage. Hamburg: CEP Europäische Verlagsanstalt.

Cabot, Heath (2016): „Refugee Voices“: Tragedy, Ghosts, and the Anthropology of Not Knowing. In: Journal of Contemporary Ethnography 45 (6), S. 1-28.

Castro Varela, Maria do Mar \& Mecheril, Paul (2010): Grenze und Bewegung. Migrationswissenschaftliche Klärungen. In: Mecheril, Paul, Castro Varela, Maria do Mar, Dirim, Inci, Kalpaka, Annita \& Melter, Claus (Hrsg.): Migrationspädagogik. Weinheim/Basel: Beltz, S. 23-53.

Christensen, Pia \& Prout, Alan (2002): Working with ethical symmetry in social research with children. In: Childhood 9 (4), S. 477-497.

Deutsche Gesellschaft für Erziehungswissenschaft (2010): Ethik-Kodex der Deutschen Gesellschaft für Erziehungswissenschaft (DGfE), http://www.dgfe.de/ fileadmin/OrdnerRedakteure/Satzung_etc/Ethikkodex_2010.pdf (Zugriff 6.6. 2017).

Deutsche Gesellschaft für Soziologie (2014): Ethik-Kodex der deutschen Gesellschaft für Soziologie (DGS) und des Berufsverbandes deutscher Soziologinnen und Soziologen (BDS), http://www.soziologie.de/de/die-dgs/ethik/ethik-kodex. html (Zugriff 6.6.2017).

Deutsche Gesellschaft für Völkerkunde (2009): Ethikerklärung der DGV, https:// www.dgv-net.de/dgv/ethik/ (Zugriff 6.6.2017).

Fichtner, Sarah \& Trần, Hoa Mai (2018): Ethische Ambivalenzen in der Forschung mit Kindern in Unterkünften für geflüchtete Menschen. In: Forum Qualitative Sozialforschung/Forum: Qualitative Social Research 19 (3), http://www.qualitative-research.net/index.php/fqs/article/view/3150/4307 (Zugriff 2.10.2018). 
Gomolla, Mechthild (2016): Diskriminierung. In: Mecheril, Paul (Hrsg.): Handbuch Migrationspädagogik. Weinheim/Basel: Beltz, S. 73-89.

Groundwater-Smith, Susan, Dockett, Sue \& Bottrell, Dorothy (2015): Participatory Research with Children and Young People. London et al.: Sage.

Guillemin, Marilys \& Gillam, Lynn (2004). Ethics, reflexivity, and „ethically important moments" in research. In: Qualitative Inquiry 10 (2), S. 261-280.

Hopf, Christel (2012): Forschungsethik und qualitative Forschung. In: Flick, Uwe, von Kardoff, Ernst \& Steinke, Ines (Hrsg.): Qualitative Forschung - Ein Handbuch. Hamburg: Rowohlt, S. 589-600.

Hugman, Richard, Pittaway, Eileen \& Bartolomei, Linda (2011): When „do no harm' is not enough: The ethics of research with refugees and other vulnerable groups. In: The British Journal of Social Work 41 (7), S. 1271-1287.

Kaukko, Mervi, Dunwoodie, Karen \& Riggs, Elisha (2017): Rethinking the Ethical and Methodological Dimensions of Research with Refugee Children. In: Zeitschrift für internationale Bildungsforschung und Entwicklungspädagogik 40 (1), S. 16-22.

Krause, Ulrike (2016): Ethische Überlegungen zur Feldforschung. Impulse für die Untersuchugn konfliktbedingter Flucht. Working Paper der Philipps-Universität Marburg https://www.uni-marburg.de/konfliktforschung/publikationen/wp 20.pdf (Zugriff 9.11.2017).

Mackenzie, Catriona, Mc Dowell, Christopher \& Pittaway, Eileen (2007): Beyond ,Do No Harm': The Challenge of Contructing Ethical Relationships in Refugee Research. In: Journal of Refugee Studies 20 (2), S. 299-319.

Norstedt, Maria \& Breimo, Janne Paulsen (2016): Moving Beyond Everyday Life in Institutional Ethnographies: Methodological Challenges and Ethical Dilemmas. In: Forum Qualitative Social Research 17 (2), http://www.qualitative-research.net/index.php/fqs/article/view/2539/3944 (Zugriff 8.8.2017).

Phelan, Shanon \& Kinsella, Anne Elizabeth (2013): Picture This ... Safety, Dignity, and Voice - Ethical Research With Children: Practical Considerations for the Reflexive Researcher. In: Qualitative Inquiry 19 (2), S. 81-90.

Pittaway, Eileen \& Bartolomei, Linda (2013): Doing Ethical Research: Whose problem is it anyway? In: Block, Karen, Riggs, Elisha \& Haslam, Nick (Hrsg.): Values and Vulnerabilities. The Ethics of Research with Refugees and Asylum Seekers. Australian Academic Press: Toowong, S. 151-170.

Punch, Samantha (2002): Research with Children - The same or different from research with adults? In: Childhood 9 (3), S. 321-341.

Stodulka, Thomas (2014): Feldforschung als Begegnung - Zur pragmatischen Dimension ethnographischer Daten. In: Sociologus 64 (2), S. 179-206.

Trần, Hoa Mai (2017): Herausforderungen im Forschungskontext mit jungen geflüchteten Kindern in Sammelunterkünften. Forschungsethische Spannungsfeder zwischen Anspruch und Wirklichkeit. Beitrag zur Veranstaltung „In erster Linie Kinder?" der Sektion Soziologie der Kindheit. In: Lessenich, Stephan (Hrsg.): Geschlossene Gesellschaften. Verhandlungen des 38. Kongresses der Deutschen Gesellschaft für Soziologie in Bamberg 2016, 
http://publikationen.soziologie.de/index.php/kongressband_2016/article/view/473 (Zugriff 11.7.2018).

von Unger, Hella, Dilger, Hansjörg \& Schönhuth, Michael (2016). Ethikbegutachtung in der sozial- und kulturwissenschaftlichen Forschung? Ein Debattenbeitrag aus soziologischer und ethnologischer Sicht. In: Forum Qualitative Sozialforschung 17 (3), http://www.qualitative-research.net/index.php/fqs/article/ view/2719 (Zugriff 8.8.2017).

von Unger, Hella, Narimani, Petra \& M'Bayo, Rosaline (Hrsg.) (2014): Forschungsethik in der qualitativen Forschung. Reflexivität, Perspektiven, Positionen. Wiesbaden: Springer VS.

White, Allen \& Bushin, Naomi (2009): More than Methods: Learning from Research with Children Seeking Asylum in Ireland. In: Population, Space and Play 17 (4), S. 326-337.

World Vision Deutschland \& Hoffnungsträger Stiftung (2016): Angekommen in Deutschland. Wenn geflüchtete Kinder erzählen, https://www.worldvisioninstitut.de/_downloads/allgemein/WorldVision_Fluchtstudie2016_web.pdf (Zugriff 2.3.2016). 


\title{
Handlungs-Spiel-Räume von Kindern in Gemeinschaftsunterkünften
}

\author{
Sarah Fichtner \& Hoa Mai Trần
}

Als wir den Hinterhof betreten, spielt Elsa ${ }^{1}$ im Sandkasten. Sie schaufelt Sand mit ihren kleinen lilafarbenen Schuhen auf die Wiese. [...] Dann steht sie auf und läuft davon, ihr Vater Fahdil hinterher. [...] Als wir alle am Tisch sitzen, erzählt Fahdil vom Aufenthalt der Familie in einer anderen Stadt in Deutschland, wo sie zwei Wochen zusammen mit Verwandten aus elf verschiedenen Städten in Deutschland und England verbracht haben. [...] Elsa sei zwar das einzige Kind gewesen, aber es sei gut für sie gewesen. Sie habe sehr viel Kurdisch gesprochen. [...] „, Guck!“, sagt Elsa zu Mai [Hoa Mai Trần , Forscherin] und zeigt zu dem Fenster in Richtung ihres Zimmers. „Essen “. „, Kocht da deine Mama?", fragt Mai. „Ja“, erwidert sie. [...] „I have bad news", unterbricht Fahdil. [...] Er zögert eine Weile, bevor er erklärt: „, They sent me Briefpost before yesterday. It's the Abschiebungsbescheid for me only. I have to go back to Bulgaria. I have one week. "Ich glaube, meinen Ohren nicht zu trauen, und auch Mai schaut fassungslos. ,"What about Kizhan [seine Frau] and Elsa?", frage ich. ,They can stay ... It's for me alleine. But I don't want to go back to Bulgaria. I don't have a life there! My life is here! My family is here. "Elsa sitzt bei Mai auf dem Schoß und schneidet kleine Motive (,,Anziehsachen“) aus Pappe aus, die sie auf ein Blatt Papier klebt. Sie ist konzentriert und fröhlich dabei, was sehr in Kontrast zu der Stimmung des Vaters steht (Feldbesuch 6, GU 1, SF, 21.7.2016) ${ }^{2}$.

Wie nah der plakativ wirkende Titel des vorliegenden Buches (,Zwischen Sandkasten und Abschiebung") den Alltagsrealitäten von geflüchteten Familien kommt, wird anhand dieser Eingangsszene deutlich: Nicht nur die beschriebene Forschungssituation spannt sich zwischen Sandkastenspiel und der

1 Alle unsere Forschungspartner*innen wurden gebeten, sich für die Publikation Namen auszusuchen. Elsa ist der selbstgewählte Name eines Mädchens in Anlehnung an die mit Zauberkräften ausgestattete Eisprinzessin aus dem Film „Frozen“.

2 Die Quellenangaben unserer Feldprotokolle beinhalten die Nummer des Feldbesuchs, benennen mit dem Kürzel GU 1 (Gemeinschaftsunterkunft 1) bzw. GU 2 (Gemeinschaftsunterkunft 2) den Forschungsort, verweisen mit SF (Sarah Fichtner) bzw. HMT (Hoa Mai Trần) auf den Namen der Autorin des Protokolls und auf das Datum der Aufzeichnung. 
Nachricht vom Abschiebebescheid ${ }^{3}$ auf. Viele Kinder, die mit ihren Eltern nach Deutschland fliehen, leben in diesem Spannungsfeld: in unsicheren, von bürokratischen Klassifizierungen des Asylstatus abhängigen Verhältnissen. Ihr politischer Status wird durch zwei entscheidende Kategorisierungen mit weiteren Unterkategorisierungen definiert: die des Kindes - in diesem Fall des jungen Kindes bis sechs Jahre - und die des Geflüchteten - und zwar hier des „geduldeten“, im Grunde „unerwünschten“ Geflüchteten mit sehr eingeschränkten Rechtsansprüchen.

Wir gehen der Frage nach, wie Kinder ihren Alltag in diesem von asylpolitischen Regelungen geprägten Kontext in Gemeinschaftsunterkünften ${ }^{4}$ leben, denn über die Lebenswirklichkeit junger Kinder in Unterkünften für geflüchtete Menschen in Deutschland ist wenig bekannt (vgl. Lewek \& Naber 2017: 11). ${ }^{5}$ Dabei interessieren uns ihre spezifíschen Lebensverhältnisse, die wir anhand ihrer ineinandergreifenden Raumerfahrungen bzw. Aneignungs- und Gestaltungspraxen von physischem/materiellem Raum, Beziehungsraum und Handlungsraum analysieren. Denn:

„A whole history remains to be written of spaces - which would at the same time be the history of powers (both these terms in the plural) - from the great strategies of geo-politics to the little tactics of the habitat" (Foucault 1980: 149).

Um dieses Zitat auf unser Forschungsfeld zu übertragen: Die „kleinen“, alltäglichen räumlichen Praktiken und Taktiken von Kindern, die uns im spezifischen Habitat der Gemeinschaftsunterkunft für Geflüchtete beschäftigen, lassen sich als Ausdruck ,großer“ geopolitischer Strategien und Machtverhältnisse im Rahmen internationaler Migrationsbewegungen verstehen. Die räumliche Perspektive ermöglicht uns, die Praktiken, Bewegungen und Entwicklungen der Kinder in ihren „mehr-als-nur-sozialen Kontexten“ zu situieren (Hackett et al. 2015: 1).

Im Folgenden werden wir unsere im Verlauf der Datenerhebung und -auswertung entwickelten Analysekategorien von physischem/materiellem Raum, Beziehungsraum und Handlungsraum einleitend anhand der oben zitierten Situation konkretisieren und in den Forschungskontext einbetten. Anschließend stellen wir unsere Fallauswahl und unsere Forschungs- und Auswertungsmethoden vor. Im Hauptteil ziehen wir die eingeführten Kategorien zur

3 Gemäß des Dubliner Übereinkommens ist Bulgarien für den Asylantrag des Vaters zuständig, da dort seine Fingerabdrücke erfasst wurden, wie er uns in einem anderen Gespräch berichtete.

4 „Ausländer, die einen Asylantrag gestellt haben und nicht oder nicht mehr verpflichtet sind, in einer (Erst)Aufnahmeeinrichtung zu wohnen, sollen in der Regel in Gemeinschaftsunterkünften untergebracht werden" (§ 53 Abs. 1 AsylG).

5 Eine aufschlussreiche ethnografische Studie zur Lebenssituation von Frauen in Unterkünften für geflüchtete Menschen in Berlin haben Dilger und Dohrn (2016) mit Studierenden der FU Berlin und dem International Women's Space publiziert. 
Handlungs-Spiel-Räume von Kindern in Gemeinschaftsunterkünften 109

Betrachtung weiterer Fallstudien heran und diskutieren abschließend die unterschiedlich gelebten und gleichsam ähnlich begrenzten Handlungs-SpielRäume der Protagonist*innen, was in Schlussfolgerungen und Forderungen mündet.

\section{Raumerfahrungsdimensionen - Herleitungen aus dem Feld}

Der physische/materielle Raum (Bewegungsraum), in dem sich das Kind unserer Eingangsszene bewegt, ist ein großer, begrünter Hof hinter der Gemeinschaftsunterkunft, der für die Bewohner*innen täglich von 7 bis $22 \mathrm{Uhr}$ zugänglich ist. Aus unseren Beobachtungen und vom Vater des Kindes wissen wir, wie wichtig dieser Außenraum für das Mädchen ist; wie viel lieber sie sich dort aufhält als im 18 Quadratmeter großen Zimmer, das sich die Familie zu dritt teilt. Wenige Unterkünfte für Geflüchtete verfügen über derartige Ressourcen und doch ist ihre Bedeutung für die Kinder wichtig - gerade aufgrund des beengten Wohnraums in den Unterkünften und ihrer Erfahrungen von Enge und Eingesperrtsein während der Flucht. ${ }^{6}$ Wie in der beschriebenen Situation läuft Elsa oft weg, wenn sie den Sandkasten oder den Hof verlassen soll. Sie veranlasst ihre Eltern, ihr hinterherzurennen, verwandelt die Situation in ein Fangspiel und nutzt die Spielmöglichkeiten, die der Hof bietet. Sie eignet sich den physisch erfahrbaren Raum an, der im Kontrast zu dem Zimmer der Familie mehr Möglichkeiten des Bewegens und Sich-von-den-Eltern-Entfernens bietet. Der physische/materielle Raum beschreibt Elsas Umfeld und ihren Aktionsradius, in dem sie lebt, sich bewegt und sich als selbstwirksam erfährt.

Hinweise auf den Beziehungsraum der Familie, der gleichzeitig auch ihre Mobilität und damit den physisch erfahrbaren Raum des Kindes erweitert, gibt der Vater, indem er von ihrem Aufenthalt bei Verwandten in einer anderen Stadt berichtet. Deutlich wird hier das transnationale Netzwerk der Familie. Ihre Zugehörigkeit zu einem sozialen Raum reicht über das lokal Begrenzte und zeitlich Gegenwärtige hinaus. Der Vater betont, wie gut es für seine Tochter gewesen sei, während des Aufenthalts bei den Verwandten Kurdisch zu sprechen. Dies kann als identitätsstiftende, von den Eltern geförderte Ressource und Verbindung zu ihrer transnationalen Gemeinschaft gedeutet werden. Elsa war bei dem Verwandtenbesuch das einzige Kind und ist es auch in

6 Elsas Vater erzählt uns, dass sie auf der Flucht in Bulgarien von der Armee aufgegriffen und für 15 Tage in ein prison gebracht worden seien. Elsa habe immerzu vor dem Fenster gesessen und geweint. Er habe die Wächter bekniet, fünf Minuten mit ihr draußen spielen zu dürfen, aber es wurde ihm nicht erlaubt. 
der beschriebenen Forschungssituation. Wir beobachten ihren routinierten Umgang mit Erwachsenen. Sie weiß, wie sie die Aufmerksamkeit auf sich ziehen kann und ist bestimmend im Beziehungsaufbau und in der Interaktion mit uns Forscherinnen, was sie durch ihre Aufforderung „Guck!“ verdeutlicht. Sie verweist auf das Zimmer der Familie im Wohnheim und auf ihre Mutter. Die Bedeutung der Beziehung zu ihren Eltern als Familie wird dadurch angesprochen und aufgrund der drohenden Familientrennung besonders relevant. Der Vater hebt hervor, dass sein Leben an das Leben mit seiner Familie gebunden ist. $^{7}$

Als uns Elsas Vater die Nachricht vom Abschiebebescheid überbringt, sitzt sie bei einer von uns Forscherinnen auf dem Schoß und bastelt. Elsa schafft sich in der Situation ihren eigenen Handlungsraum durch Spiel, was auch in der Raumnutzung des Hofs deutlich wird. Während die Erwachsenen aufgrund der überbrachten Nachricht fassungslos sind und sich ein Gefühl der Handlungsohnmächtigkeit einstellt, ist sie kreativ tätig. Der Handlungsraum wird von uns als Möglichkeit, Fähigkeit und Vermögen des Handelns (Agency) in einem spezifischen Kontext - hier bildlich gesprochen zwischen Sandkasten und Abschiebung - verstanden.

Mit unserem kinderzentrierten, auf alltägliche Raumerfahrungen und -aneignungspraxen gerichteten Blick (vgl. Hackett et al. 2015; Rasmussen 2004) möchten wir angesichts erheblicher struktureller Diskriminierungen von Kindern mit unsicherem Aufenthaltsstatus (vgl. Eisenhuth 2015) ihre Akteur*innenschaft in diesen besonderen Lebensverhältnissen hervorheben und „Opferdiskurse" oder defizitär-homogenisierende Narrative über geflüchtete Kinder herausfordern (vgl. Kaukko et al. 2017).

Der häufig beschriebene asylrechtliche „Wartezustand“ suggeriert eine statische Lage: das Warten auf eine asylrechtliche Entscheidung (vgl. Seeberg et al. 2016: 400; Lewek \& Naber 2017: 6). Wir haben die Kinder, mit denen wir forschten, als aktiv erlebt. Die Kinder zeigen vielfältige Handlungsspielräume auf, gehen Routinen nach, gestalten ihren Alltag und ihr Umfeld mit, trotz institutioneller Einschränkungen und unterschiedlicher Reglementierungen. Die Vielschichtigkeit ihrer Handlungs-Spiel-Räume ist in dieser von uns gewählten Begrifflichkeit angelegt: physischer/materieller Raum (Bewegungsraum), Beziehungsraum und Handlungsraum sowie der mit allen drei Raumerfahrungen verknüpfte Raum für Spiel, bedingen, (v)erschließen und beeinflussen sich gegenseitig, sind miteinander verwoben. Sie stellen einen offenen und ergiebigen Referenzrahmen von Möglichkeiten und Begrenzungen unterschiedlicher Raumerfahrungen dar, welcher Orientierung gibt, um die Lebenssituation jeder einzelnen Familie mit ihren je eigenen Themen abzubilden.

7 Eine Familientrennung steht im Widerspruch zur Möglichkeit der Familienzusammenführung und zu den Artikeln 3, 5, 7 und 8 der UN-Kinderrechtskonvention. Auch nach $\S 1684$ BGB Abs.1 hat jedes Kind ,das Recht auf Umgang mit jedem Elternteil““. 


\section{Vom Spielen in Räumen zu Handlungsspielräumen: Fallauswahl und Methoden}

Wir führten unsere Forschung zwischen Mai 2016 und Februar 2017 in zwei Gemeinschaftsunterkünften in Berlin durch. Sowohl die Wahl dieser Unterkünfte, wie die Wahl der Forschungspartner*innen erfolgte feldgeleitet, das heißt von sieben angefragten Unterkünften stimmten zwei unserer Forschung zu. Die zwei Gemeinschaftsunterkünfte, in denen wir forschten, wurden von gemeinnützigen Trägern betrieben. Sie unterschieden sich in Bezug auf ihren Standort in Berlin (zentrale versus eher periphere Lage), die Dauer der Inbetriebnahme (seit vielen Jahren versus seit ein paar Monaten) und hinsichtlich der Größe und Beschaffenheit ihrer Räumlichkeiten: Die erste Unterkunft beherbergte im Forschungszeitraum ca. 150 Personen (darunter über 20 Kinder) in Einzelzimmern mit Gemeinschaftstoiletten und -duschen auf vier Etagen eines Altbaus; die zweite ca. 500 Personen (darunter über 170 Kinder) auf mehreren Etagen in Zweizimmerwohnungen mit Bad in einem Neubau.

Von sieben angefragten Familien mit Kindern unter sechs Jahren gaben drei ihre Einwilligung an unserer Studie teilzunehmen. ${ }^{8}$ Drei Kinder dieser Familien, Elsa (drei Jahre), Lara (drei Jahre) und Spiderman (sechs Jahre), sind die Protagonist*innen dieses Beitrags.

Wir verfolgten eine explorative, ethnografische Forschungsstrategie (vgl. Breidenstein et al. 2015), um möglichst dichte, vielschichtige Informationen über die Lebensverhältnisse unserer Forschungspartner*innen zu generieren. So verbrachten wir viel Zeit mit teilnehmender Beobachtung: der Spielbeobachtung der Kinder, dem aktiven Mitspielen und mit „kindzentrierten Methoden" (Christensen \& Prout 2002: 480; White \& Bushin 2011), wie zum Beispiel kindergeleiteten Hausbegehungen.

Die Herausforderung in der Forschung mit besonders jungen Kindern bestand darin, sich auf die Interessen und Ausdrucksmöglichkeiten jedes Kindes individuell einzulassen, was eine hohe methodische Flexibilität und ein spielerisches Ausprobieren erforderte (White \& Bushin 2011: 326). Mit Elsa wurden beispielsweise viele Orte in der Unterkunft einsehbar, alltägliche Praxen und Beziehungen wurden für uns ersichtlich und methodisch konnte einiges kreativ ausprobiert werden. Mit Lara forschten wir vorrangig im Kinderbetreuungsraum und beobachteten ihr vielseitiges Spielverhalten und ihre Interaktionen mit uns. Viele Informationen erhielten wir weiterhin im Gespräch mit ihrer Mutter. Bei Spiderman lernten wir durch ein gezieltes Begleiten seine präferierten Tätigkeiten kennen und damit, wo er sich aufhielt und was ihm wichtig war.

8 Zur Problematik des Feldzugangs und -exits im Forschungsprojekt siehe die Beiträge von Trần und Scott sowie auch Dilger \& Dohrn 2016. 
Unsere Beobachtungen ergänzten wir durch Gespräche - zum Teil mithilfe einer Sprachmittlerin - mit Kindern, Eltern und Mitarbeiter*innen sowie durch formale, leitfadengestützte und aufgezeichnete Interviews mit Heimleitungen und Mitarbeiter*innen. Unser Datenkorpus umfasst 24 Feldprotokolle, darunter auch E-Mail-Korrespondenzen mit einer abgeschobenen Familie.

Während des Forschungsprozesses diente das bereits Beobachtete und Aufgezeichnete im Sinne einer ,zirkulär angelegte[n] Forschungspraxis“ (Breidenstein et al. 2015: 9) der ständigen (Weiter-)Entwicklung unserer Forschungsfragen und -erkenntnisse: Vom gemeinsamen Spiel in den Räumen der Unterkünfte kamen wir auf Fragen der unterschiedlichen Raumerfahrungen und -nutzungsmöglichkeiten unserer Forschungspartner*innen, die wir infolge expliziter - mit Fokus auf ihre Handlungen - untersuchten. Dabei war auffällig, dass die Zugänglichkeit von Räumen durch soziale Beziehungen ermöglicht oder begrenzt wurde, welche in weiteren Schritten näher betrachtet wurden.

In einem ersten Schritt wurden unsere Feldprotokolle in eine Software für qualitative Datenanalyse eingespeist und offen kodiert. In einem zweiten Schritt entwickelten wir vergleichende, übergeordnete Kodes, durch die (für uns) zentrale Themen markiert wurden. In einem dritten Schritt erarbeiteten wir anhand besonders dichter Situationen unsere drei Analysekategorien: physischer/materieller Raum, Beziehungsraum, Handlungsraum.

Die differenzierte und ,kontrastierende Perspektive“ der Fallstudienarbeit (Bollig et al. 2016: 12) rahmt auch diesen Text und findet sich in den Typologien wieder, die unsere Abschnitte bezeichnen: Elsa: die selbstbestimmte Macherin, Lara: die lernende Perfektionistin, und Spiderman: der Rebell im Zwischenraum. Mittels dieser Fallstudien bleiben wir nah an den Forschungspartner*innen und ihren jeweiligen Kontexten, zeigen ihre kontrastiven Raumerfahrungen und -aneignungspraxen und machen zugleich auch die Vielfalt an Kindheiten in verschieden räumlich strukturierten Unterkünften für geflüchtete Menschen sichtbar.

Diese in den Unterkünften gelebten Kindheiten werden durch bürokratische Klassifizierungen, die den Aufenthaltsstatus der Familien mitbedingen, beeinflusst, was wiederum Folgen für den Forschungsablauf und die Rolle der Forscherin haben kann (wie in der Eingangsszene geschildert). Die Forscherin wird mitunter zur Grenzgängerin zwischen Erhebungsinteressen und Handlungserfordernissen des Feldes, sie muss sich beispielsweise in der eingangs beschriebenen Situation zur Abschiebenachricht des Vaters verhalten. ${ }^{9}$ Diese Situation stellte im Forschungsprozess keinen Einzelfall dar: Alle drei

9 Eine Reflexion darüber, was wir mit dem Forschungsfeld und was das Feld mit uns macht, welchen Raum wir in diesem von Machtstrukturen und Ungewissheiten durchsetzten Rahmen einnehmen und was wir ermöglichen, begleitet dementsprechend unsere Analyse und wird im Beitrag von Trần und in Fichtner \& Trần (2018) aufgegriffen. 
Handlungs-Spiel-Räume von Kindern in Gemeinschaftsunterkünften 113

Familien, mit denen wir forschten, verfügten zum Forschungszeitpunkt über keine sichere Aufenthaltsperspektive in Deutschland. ${ }^{10}$

\section{Die Erfahrungsräume und Praktiken der Kinder}

Von unserer exemplarischen Eingangssituation ausgehend, anhand derer wir unseren Forschungskontext und unsere Analysekategorien eingeführt haben, werden wir im Folgenden mittels unserer drei Fallstudien weiter ausführen, wie unterschiedliche von Kindern erlebte und mitgestaltete Erfahrungsräume ihre Lebensverhältnisse in Gemeinschaftsunterkünften prägen. Jedes Kind stellt die Hauptfigur einer Fallstudie dar. Dabei gehen wir von Kindern als tätigen Akteur*innen aus, die nicht erst jemand werden, sondern schon jemand sind (Hackett et al. 2015: 1).

\subsection{Elsa: Die selbstbestimmte Macherin}

Elsa, die Protagonistin unserer Eingangsszene, ist zu Beginn der Erhebung drei Jahre alt und befindet sich seit 14 Monaten mit ihren Eltern in Deutschland. Sie lebt mit ihrer Mutter (Kizhan) und ihrem Vater (Fahdil) in einem Wohnheim für geflüchtete Menschen in einem ca. 18 Quadratmeter großen Zimmer. Ihr Aufenthaltsstatus ist ungeklärt und über die Zuständigkeit ihres Erstantrags aufgrund des Dublin-Verfahrens noch nicht entschieden. Die Eltern geben an, aus dem Nordirak zu kommen, und bezeichnen sich als Kurden. Die Familie spricht Sorani (Kurdisch), Arabisch, Türkisch, Englisch und Deutsch. Elsa besucht zum Zeitpunkt der Forschung eine Kindertageseinrichtung. Am Abend der eingangs beschriebenen Szene sind wir im Zimmer der Familie zum Essen eingeladen:

Am Fenster stehen rechts das Doppelbett der Eltern und links daneben das Kinderbett von Elsa mit einem großen Teddybär; davor ein kleiner Kindertisch mit Kinderstuhl; daneben ein Schrank, auf dem sich viele Koffer türmen. An der linken Wand ist eine kleine Kochnische mit einem Waschbecken, an dem Kizhan beschäftigt ist. [...] Sie hat auf dem Zimmerboden vor den Betten eine Folie ausgebreitet, auf der ein Teil der Speisen bereits angerichtet ist. [...] Wir setzen uns auf den Boden, um nicht im Weg zu stehen. Im Fernseher oben auf einer Kommode laufen leise „Die Simpsons“. [...] Elsa isst nicht viel, sondern

10 In Elsas Fall bedingt durch das Prüfen der Zuständigkeiten innerhalb des Dublin-Verfahrens; in den zwei anderen Fällen bedingt durch die Ablehnung des Asylantrags. 
will lieber spielen. Sie ist sehr aktiv, was im Kontrast zu der Schwermut der Eltern steht. [...] Sie springt auf dem Bett auf und ab und ruft immer wieder: „,Guck, Mai!“" Dann will sie etwas malen und verteilt all ihre Stifte im Bett. Kurz darauf zieht sie an meinen Armen, möchte, dass ich auch auf das Bett komme. Ich ziehe auch an ihren Armen, so dass wir hin und her schaukeln, was sie sehr lustig findet. [...] Mai ist bereits mit Essen fertig und bietet Elsa an, mit ihr zu malen bzw. zu schreiben. „Komm, sag mir, was ich schreiben soll“, fordert sie Elsa auf. Elsa diktiert Namen: „Elsa, Kizhan, Fahdil, Sarah [Sarah Fichtner, Forscherin], den Namen der Kinderbetreuerin ... Mai " (Feldbesuch 6, GU 1, SF, 21.7.2016).

Die beschriebene Räumlichkeit markiert den beengten, multifunktionalen Privatraum der Familie. Er dient als Schlafzimmer, in dem Kind und Eltern in separaten Betten schlafen. Des Weiteren wird er als Küche durch die Kochnische und als Esszimmer durch eine auf dem Boden ausgebreitete Folie verwendet. Als Wohnzimmer mit Fernseher dient der Raum der medialen Unterhaltung. Stauraum gibt es in und auf dem Schrank. Die Enge des Raumes wird spürbar, als wir uns auf den Boden setzen, um der Mutter bei der Zubereitung des Essens nicht im Weg zu stehen. Das Elternbett wird von Elsa als Spielraum verwendet. Sie setzt sich zum Malen nicht an den kleinen für sie bestimmten Tisch, sondern nutzt die vergleichsweise größere Fläche des Bettes. Sanitäranlagen sowie größere Kochmöglichkeiten werden auf jeder Etage der Unterkunft gemeinschaftlich genutzt. ${ }^{11}$

Elsa interagiert während des Essens spielerisch mit uns Forscherinnen. Die Ressource des Besuchs erweitert ihren Spiel-, Handlungs- und Interaktionsraum, während die Eltern von uns als schwermütig wahrgenommen werden. Im selbstgewählten Namensdiktat gibt Elsa einen Einblick in ihre Beziehungswelt: Sie nennt erst sich, dann ihre Eltern, gefolgt von den Namen von uns Forscherinnen und der Kinderbetreuerin der Unterkunft. Elsas Vater erzählt, dass sie diese und eine technische Hilfskraft sehr möge, auch wenn wir ihre Interaktionen mit der Kinderbetreuerin selten beobachten konnten. Neben ihrer Kernfamilie erweitern die Mitarbeiter*innen (z. B. auch Sozialarbeiter*innen) der Unterkunft Elsas soziales Netzwerk und ihren alltäglichen Bewegungsraum sowie ihre Handlungsmöglichkeiten außerhalb des Privatraums der Familie. Die Beziehungen ermöglichen ihr beispielsweise den Zutritt in das Mitarbeiter*innenzimmer, ins Frauencafé oder in den abgeschlossenen Kellerbereich. Elsa verwickelt die Erwachsenen, so auch uns, in spielerische Aktivitäten und gestaltet ihren Beziehungsraum aktiv. Neben ihren Beziehungen zu Erwachsenen hat Elsa sieben Freunde (darunter eine beste Freundin) in der Kita.

11 Elsas Eltern beschweren sich mehrmals über die hygienischen Bedingungen und die Wartezeiten für Dusche und Toilette. Elsa kommentiert bei einer Raumbegehung, als auf die Toilette gezeigt wird: „Bäh!“ Die Eltern erzählen, dass Elsa in der Unterkunft Windeln tragen müsse, was für die Zeit in der Kita nicht zutreffe. 
Die Abendessensituation, die wir als von der Nachricht des Abschiebebescheids geprägt wahrnehmen, beobachten wir auch als Spielsituation, was der folgende Protokollauszug verdeutlicht:

Ich bin noch von der Abschiebenachricht vereinnahmt [...]. Elsa bekommt das alles mit. Sie ist viel im Zimmer unterwegs. [...] Sie nimmt beispielsweise ein Brötchen, steckt es auf eine Gabel und die Gabel dann in eine Schrankritze. Dort hängt dann ein Brötchen, was irgendwie witzig ist. Der Vater ermahnt sie sofort, ruft direkt nach der Mutter, sagt noch „Bitte“ zu Elsa und die Mutter „Nein“ zu ihrer Handlung. Sie sprechen auf Kurdisch mit dem Kind. [...] Elsa spielt mit dem Küchenpapier. Ich zeige ihr, wie man es als Fernglas benutzen kann und trete durch die Küchenrolle immer wieder in ihr Sichtfeld, was sie zum Lachen bringt. [...] Sie rennt wieder zum Bett, springt darauf herum, ist voller Energie. Ihre Mutter meint, dass sie eigentlich schon müde sein müsse, da es gegen 19 Uhr geht. Doch Elsa zeigt keine Anzeichen von Müdigkeit (Feldbesuch 6, GU 1, HMT, 21.7.2016).

Elsas Bewegungsdrang steht in Kontrast zu der räumlichen Enge des Privatraums. Sie rennt, springt und bewegt sich nahezu ruhelos, was die Mutter als ungewöhnlich für die Uhrzeit einschätzt. Elsa eignet sich den Raum kreativ an und zweckentfremdet Gegenstände eigensinnig, um sich neue Spielmöglichkeiten zu schaffen. Lebensmittel, Besteck und Küchenpapier werden in ihren Spielrahmen integriert. Zugleich rebelliert sie damit gegen die normativen $\mathrm{Zu}-$ schreibungen - und damit Handlungsbegrenzungen - der Erwachsenen, wie bzw. wofür etwas zu nutzen sein soll.

Durch ihre vielen und unterschiedlichen Tätigkeiten fordert sie auch die Reaktion der Eltern und Forscherinnen heraus. Sie gestaltet den Beziehungsraum und die soziale Situation, die sich für uns im Spannungsverhältnis von zukünftiger Abschiebung und gegenwärtigem Spiel bewegt. Unsere beschriebene Anteilnahme an dieser Situation und die eher bedrückte Stimmung durchbricht Elsa durch humorvolle, provozierende und aufmerksamkeitserregende Momente und durch Spiel. Damit wird ein Kontrapunkt zum Empfinden der Lähmung bzw. Machtlosigkeit der Erwachsenen gesetzt, der mit der angekündigten Abschiebung verbunden ist.

An einem anderen Tag gehen wir mit Elsa in den Kinderbetreuungsraum der Unterkunft. Laut Aushang ist die Betreuung drei Mal pro Woche für drei Stunden geöffnet, wird aber primär nach Bedarf genutzt, da die Einrichtung zum Forschungszeitpunkt nicht viele Kinder beherbergt, die diese Betreuung in Anspruch nehmen. Einige sind zu jung, andere gehen, wie Elsa, in die Kita oder in die Schule.

Wir malen mit Elsa. Bald darauf kommt Fahdil [Elsas Vater] ins Zimmer. Er geht auf Sarah und mich zu. Wir sitzen auf kleinen Stühlen am Tisch. Elsa geht direkt zu ihrem Vater und sagt laut: „,DU BIST RAUS! “, und drückt ihn zurück (Feldbesuch 7, GU 1, HMT, 28.7.2016). 
Der Sozialarbeiter der Unterkunft berichtet, dass die Eltern im „Schockzustand" seien. Die innerfamiliale Dynamik zeigt sich in der Spielsituation als ambivalente Aushandlung von Nähe und Distanz. Als sich der Vater uns nähert, geht Elsa auf ihn zu, verteidigt ihr räumliches Territorium und bekräftigt ihren Anspruch auf die (ungeteilte) Aufmerksamkeit der Forscherinnen. Sie spricht ihn auf Deutsch an und drängt ihn mit ihrem Körper zurück. Als Konsequenz verlassen wir mit dem Vater den Spielbereich. Er erzählt von der schwierigen familialen Lage und dass die Familie - um zusammenzubleiben bald untertauchen müsse. Bei unserem darauffolgenden Besuch präsentiert sich uns eine ähnliche Szene, als wir mit Elsa kneten:

Ihr Vater betritt den Raum. Elsa unterbricht ihr Spiel und sagt zu ihm: „Papa! Raus spielen!“ Er verneint, und sie fährt fort: „Papa, nicht raus. Ja. Raus, geh spielen! Papa, komm hier. Gut, ja. "Sie hält ihn in ihrer Nähe und meint zu uns: „Des ist meine Papa“ (Feldbesuch 8, GU 1, HMT, 9.8.2016).

Auch in dieser Situation fordert Elsa ihren Vater zunächst auf, den Raum zu verlassen, um ungestört mit uns Forscherinnen zu spielen. Gleichzeitig verdeutlicht sie die familiale Zugehörigkeit und Nähe zu ihrem Vater, indem sie ihn auffordert, zu ihr zu kommen, und uns gegenüber verbalisiert, dass er ihr Vater sei. Wir nehmen dies als Ambivalenz wahr, welche ihr Bedürfnis nach Nähe zu ihrem Vater und gleichzeitig nach situativer Distanz und Abstand von der familiär belastenden Lage sucht, die sie möglicherweise durch ihr Spiel zu kompensieren versucht.

Nachdem der Vater den Raum verlassen hat, spielt Elsa ausgelassen, fast hektisch in der Spielküche. Als sie die vielen unterschiedlichen Spielmöglichkeiten ausgeschöpft hat, drängen wir auf eine Aufräumpause, welche Elsa wiederholt mit den Worten „Nein, es ist nicht Aufräumzeit“ negiert. Sie besteht auf ihrer Spielzeit im Zimmer, bestimmt ihre Spieltätigkeiten selbst, beginnt verschiedene Tiere und Formen zu kneten, zerschneidet die Knete und verbalisiert das mit ,autschi, autschi“. Sie fordert sich Malfarbe aus einem abgeschlossenen Schrank ein und malt und übermalt aufgeregt und schnell ein Blatt mit roter Farbe. Am Ende der Spielsituation zeigt sich Elsa in ihrer Aktivität unruhig. Wir nehmen sie als aufgewühlt wahr.

Ihr Vater betritt erneut das Zimmer. Er beschließt, Elsa ins Bett zu bringen, meint noch, dass sie mitten in der Nacht aufgewacht sei und viel geweint habe. Er wisse auch nicht, was los sei. Die Kinderbetreuerin [...] hatte uns gesagt, dass Fahdil und Elsa sich vier Mal hintereinander „Frozen-Die Eiskönigin " - zweimal auf Deutsch und zweimal auf Englisch - angesehen hätten, weil Elsa es unbedingt so gewollt hatte. Elsa fängt wieder an zu weinen, ist überdreht. Fahdil sagt: „,Muss schlafen“, und trägt sie weg. Wir räumen auf und klopfen an die Zimmertür, um uns zu verabschieden. Elsa liegt mit einer Nuckelflasche, die mit Milch gefüllt ist, im Bett (Feldbesuch 8, GU 1, HMT/SF, 9.8.2016). 
Diese Abschlussszene setzt Elsas Tätigkeit und Bestimmtheit Grenzen. Das gemeinsame Aufräumen wird ausgesetzt. Es ist ca. 17 Uhr. Die Routine des Alltags scheint durchbrochen. Nachts hat Elsa wenig geschlafen und am Tag schwankt sie zwischen gesteigerter Aktivität und emotionaler Niedergeschlagenheit. Möglich ist, dass das gemeinsame Filmschauen eine Beruhigung und Ablenkungsmöglichkeit darstellt und einen imaginären Raum schafft, der die konkrete Lebensrealität temporär zurückdrängt. Elsa, die sonst häufig lachend wegrennt, wird nun weggetragen. Bei der Verabschiedung liegt sie still im Bett und trinkt Milch aus einer Nuckelflasche. Die Abschlussbegegnung und die Anfangssequenz zeigen unterschiedliche Seiten an Elsa. Sie verdeutlichen, dass bei ihren aktiven Tätigkeiten und selbstbestimmten Grenzsetzungen gegenüber Erwachsenen auch ihre Grenzen und ihre Bedürftigkeit gesehen werden müssen. Der Vater ermöglicht ihr, im Kinderbett in klar begrenztem Raum zu sein, den Elsa sich nicht erst aktiv erobern muss. Vielmehr kann sie sich ganz auf ihre Bedürfnisbefriedigung (Nuckelflasche) zurückfallen lassen. Elsa bleibt von der schwierigen Familiensituation nicht unberührt, und es wird deutlich, dass auch ihre Bewältigungsressourcen endlich sind.

Nachvollziehbar wird auch, wie verschränkt ihre von uns analysierten Raumerfahrungen sind und wie sie sich gegenseitig bedingen: Elsa schafft sich aktiv Handlungsspielräume über ihre Beziehungen zu Peers und Erwachsenen. Die Familie ist sehr mobil, verfügt über einen Bewegungsradius, der über die Unterkunft hinausgeht, und einen erweiterten Beziehungsraum: ein transnationales Netzwerk, gute Beziehungen zu den Mitarbeiter*innen der Unterkunft, $\mathrm{zu}$ den Forscherinnen und weiteren Bekanntschaften. Elsa erschließt und gestaltet sich durch ihre soziale Interaktionsbereitschaft Beziehungen (,,doing relations") und nutzt Räume für ihre eigenen Interessen - verteidigt sie sogar. Sie verhält sich nicht nur zu ihren Lebensverhältnissen, sondern versucht, auf diese Einfluss zu nehmen und die bestehenden Spielräume zu erweitern. Außer Acht gelassen werden sollten dabei nicht die Begrenzungen, die sie erlebt: Neben der räumlichen Enge des Privatzimmers, den sanitären Bedingungen geteilter Toiletten und dem eingeschränkten Zugang zu weiteren Räumen stellt die fehlende Aufenthaltsperspektive eine emotionale Belastung dar, die das Wohlbefinden der Familie gravierend einschränkt.

Nach wöchentlichen Besuchen, die sich über einen Zeitraum von ungefähr einem Monat erstrecken, taucht die Familie für ungewisse Zeit bei Verwandten unter. Es entsteht ein abrupter Forschungs- und Beziehungsabbruch. Wir stehen über Textnachrichten in Kontakt, der aber durch einen Telefonnummernwechsel endet. Wir erkundigen uns bei der Einrichtung nach der Familie und sehen sie nach elf Monaten wieder. Die Familie konnte nach Ablauf der Überstellfrist im gleichen Zimmer unterkommen und sucht wie zu Beginn der Erhebung eine eigene Wohnung. Der Vater erzählt von Diskriminierungen bei der Wohnungssuche. Die Mutter ist im fünften Monat schwanger und Elsa 
betont eindrücklich, sich auf ihren jüngeren Bruder zu freuen. Sie spielt aufgeweckt im Hof des Wohnheims.

\subsection{Lara: Die lernende Perfektionistin}

$\mathrm{Zu}$ Beginn unserer Forschung im Juli 2016 hat Lara Geburtstag und wird drei Jahre alt. Sie bewohnt mit ihrer Mutter (Sandra) und ihrem Vater im selben Wohnheim wie Elsa ein ebenfalls ca. 18 Quadratmeter großes Zimmer. Die Mutter gibt an, aus Bosnien zu kommen, wo sie vier weitere Töchter im Alter von sechs, 12, 14 und 16 Jahren habe. Sie bezeichnet ihre Familie als ,Zigeuner" und sich als Christin. Sie spricht Deutsch und sagt, dass sie auch mit Lara Deutsch spreche, der Vater Bosnisch. Mit ihm haben wir während der Forschungszeit kaum Kontakt. Lara spricht ihre eigene Sprache, etwas Englisch und etwas Deutsch. Sie geht seit Anfang 2016 für zwei Stunden am Tag in eine Integrationskita. Mutter, Vater und Kind sind seit November 2014 in Deutschland. Ihr erster Asylantrag wurde abgelehnt, während der Forschung wartet die Familie auf die Entscheidung des Zweitantrags. Wir treffen Lara mit ihrer Mutter Sandra zum ersten Mal im Kinderbetreuungsraum der Unterkunft:

Sandra kommt mit Lara auf dem Arm den Gang entlanggeeilt. [...] Sie lässt sie in der Tür des Kinderbetreuungsraums auf den Boden, und Lara rennt los, sichtlich begeistert. [...] Sie scheint alles Spielzeug ausprobieren zu wollen. Immer wieder ruft sie ,, Wow! " und die Mutter erklärt uns, das habe sie aus den englischen Lerncartoons, die sie sich mit Vorliebe anschaue. Die deutschsprachigen Cartoons [...] möge sie nicht. Also lerne sie eher englische als deutsche Wörter. Lara redet vor sich hin [...]. Mai fragt, ob das Bosnisch sei. Die Mutter verneint. Sie habe ihre eigene Sprache, in der ",Aboi “ zum Beispiel für ,Baby“ stehe. „Ich lerne auch Laras eigene Sprache“, erklärt sie uns lächelnd. [...] „,Sie spielt immer für sich alleine“, fügt sie hinzu. ,, Wenn zu viele Kinder dazukommen, geht sie wieder. Sie will lieber alleine sein " (Feldbesuch 5, GU 1, $S F$, 13.7.2016).

Anhand dieser Szene beobachten wir, wie begeistert und explorativ sich Lara den Kinderbetreuungsraum erobert. Sobald sie von ihrer Mutter Sandra abgesetzt wird, erkundet sie in Bewegung die Möglichkeiten des Raums und verleiht ihrer positiven Raumerfahrung durch den staunenden Ausruf „Wow!“ Ausdruck. Die Mutter erklärt uns, dass Lara erst zum zweiten Mal da sei und es daher viel Neues für sie zu entdecken gebe. Die Forschungssituation ermöglicht Lara somit eine neue Raumerfahrung, einen eigenen Spielraum für sich 
selbst. ${ }^{12}$ Laras Spieltätigkeit zieht sich durch den gesamten Forschungsprozess hindurch und verdeutlicht ihre aktive Auseinandersetzung mit sich und ihrer Umwelt als lernenden Prozess. Wir beobachten im weiteren Verlauf der Forschung ihr eigenständiges Spiel, das selbstbestimmte Verlassen der Forschungssituation und interpretieren es als Ausdruck ihrer Handlungsfähigkeit, ihre eigenen Bedürfnisse zu erkennen und entsprechend zu handeln.

Wir Forscherinnen bleiben mit der Mutter sitzen und beobachten ihr selbstständiges Spiel. Sandra kommentiert und kontextualisiert Laras Handlungen; auch um ihr Tun aus ihrer Perspektive uns Forscherinnen zu erklären. Ihre Bereitschaft, Laras eigene Sprache zu lernen, die genaue Beobachtung ihrer Tochter und das Eingehen auf ihre Spielhandlungen und -vorlieben zeichnen ihre Beziehung aus. Zu Laras Beziehungsnetzwerk berichtet uns ihre Mutter später, dass sie den Erzieher ihrer Kita möge. Im Heim distanziere sich die Familie von den anderen Bewohner*innen. Unter den Mitarbeiter*innen ist lediglich eine technische Hilfskraft für Lara als Spielpartner relevant. Sandras Erläuterungen suggerieren, dass Lara andere Kinder eher meidet und lieber alleine ist.

Nach anfänglichen Unsicherheiten, wie wir am besten eine Interaktion mit Lara aufbauen, wird später am selben Tag ein erster „Kommunikationsdraht“ gefunden:

Lara sitzt auf der Hochebene mit dem Plastiktelefon in der Hand. Sandra erklärt: „Ich hab ja noch vier Töchter in Bosnien, mit denen telefoniert sie. Auch auf der Straße geht sie in Telefonzellen. Das mag sie. Das kennt sie aus den Cartoons. "Ich weiß nicht, wo Sarah das andere Kastentelefon her hat [...]. Sie nimmt den Hörer ab, nimmt Blickkontakt zu Lara auf und sagt laut in den Hörer: ,Hallo, Lara!“ Lara ist kurz irritiert, schaut zu ihr herunter, geht dann aber auf Sarahs Kommentare und Fragen in ihrer eigenen Sprache ein (Feldbesuch 5, GU 1, HMT, 13.7.2016).

Der Beziehungsaufbau von uns Forscherinnen zu Lara wird aus der Distanz aufgebaut. Das Telefonieren, das Lara laut ihrer Mutter aus Cartoons, über die Telefonate mit ihren Schwestern in Bosnien und dem Spiel in öffentlichen Telefonzellen kennt, stellt ein Medium dar, um mit ihr in Interaktion zu treten. Das „Gespräch“ wird durch ein „Hallo“ und „Tschüss“ gerahmt. Die Möglichkeit, mit uns zu „telefonieren“, wird am selben Tag und bei darauffolgenden Besuchen immer wieder von Lara in Anspruch genommen und auch von ihr selbst initiiert. Wir beobachten, dass Lara das immer gleiche Plastikmobiltelefon von derselben Stelle nimmt, zum Telefonieren auf die Hochebene des Raumes geht, beim Auflegen routiniert nach unten geht und das Telefon wieder an

12 Dabei tritt einmal die Situation ein, dass für uns und Lara der Kinderbetreuungsraum geräumt wird und wir damit andere Kinder von der Raumnutzung ausschließen. Somit waren wir an Exklusions- und Inklusionsmechanismen und der Nutzbarmachung und Verschließung von Ressourcen der Unterkunft beteiligt. 
die gleiche Stelle hängt. Lara schafft es, ihre eigenen Interessen durch die eigensinnige Struktur ihres Spiels einzulösen und stellt eine habituelle Ordnung in ihren Spielabläufen her, welche zugleich Nähe schafft und Distanz hält.

Auf unsere Frage nach der Alltagsstrukturierung erzählt die Mutter, dass es ,jeden Tag das Gleiche“ sei. Sie sagt:

„Es ist sehr schwer mit Lara, wir sind die ganze Zeit hier", und meint, dass sie wegen Lara keinen Besuch empfangen könne, da Lara Personen aus der Tür stoße [...]. , Auf jeder Etage gibt es einen Duschraum. Lara duscht gerne. Sie zieht mich dann an der Hand und dann geht das stundenlang. Sie mag Wasser [...,] nach der Kita gibt's Mittagessen, dann spielt sie draußen auf dem Spielplatz oder im Zimmer. Danach macht sie Mittagsschlaf. [...] Es gibt nur einen Spielplatz, den sie mag “ (Feldbesuch 5, GU 1, SF, 13.7.2016).

Der Bewegungsraum von Lara folgt einem routinierten Tagesablauf. Das Aufsuchen fester Orte beschränkt die Nutzung weiterer Räume und Ressourcen. Die Möglichkeiten des Außenraums der Unterkunft (die Heimleiterin spricht uns gegenüber vom Kiez als einer ,grünen Insel“) können von Lara nur begrenzt genutzt werden. Die Mutter gibt an, die meiste Zeit im Privatraum der Unterkunft zu verbringen. Besuch lasse Lara nicht zu. Das Duschen wird als ausgedehnte, präferierte Tätigkeit beschrieben. Die gemeinsam mit anderen Bewohner*innen zu nutzenden Toiletten suche Lara nicht auf, da sie Windeln trage. Der Kinderbetreuungsraum und der Hof der Unterkunft gehörten nicht zu ihren gewohnten Spielorten. Die Mutter beschreibt die Kita und einen bestimmten Spielplatz als Bewegungsräume außerhalb der Unterkunft. Innerhalb dieses Aktionsradius nehme Lara die Grundbedürfnisse nach Schlaf, Essen, Sauberkeit und Spiel wahr. Einen Umzug in eine Wohnung lehnt Sandra aufgrund der Aufrechterhaltung gewohnter Tagesabläufe ab. Der Handlungsraum der Mutter ist durch Laras Handlungs- und Bewegungsraum bedingt und spiegelt die Beziehung von Mutter und Tochter wider:

Sie kämen überall zu spät, zu den Arztbesuchen zum Beispiel. In der U-Bahn sei es ganz schwer. Ich merke an, dass dies ja auch für die Mutter eine große Einschränkung ihrer Mobilität bedeute. „Ja, einmal wollte sie zwei Monate nicht raus [nachdem Lara eine Spritze beim Arzt bekommen hatte]. Und alles muss immer an seinem Platz stehen. Wir können nichts wegwerfen, auch nichts verrücken, nicht mal den Teppich. Sie ist Perfektionistin " (Feldbesuch 5, GU 1, $S F$, 13.7.2016).

Laut dem Bild, das ihre Mutter von Lara zeichnet, erfordere Laras Gesundheit Besuche bei Ärzt*innen, die ihren routinierten Tagesablauf durchbrechen. Die Nutzung öffentlicher Verkehrsmittel überfordert sie; der Erhalt von Spritzen greift stark in ihr Wohlbefinden ein: Danach zieht sich Lara in das Bewohner*innenzimmer zurück und will es nicht verlassen. Laras von der Mutter angesprochenen Perfektionismus nehmen wir auch im Kinderbetreuungsraum wahr, in dem wir unsere wöchentliche Forschungszeit mit ihr verbringen. Sie 
verrückt oft Gegenstände und perfektioniert die Raumanordnung nach ihren eigenen Vorstellungen. Die Ordnung, die sie dabei selbst herstellt, um alles an einem festen Platz stehen zu haben, verschafft ihr Orientierung und strukturiert ihr Spiel. Einen Zutritt in das Bewohner*innenzimmer gewährt uns die Mutter nicht, was wir als Wahrung der familiären Privatsphäre respektieren.

Als Laras Mutter bei späteren Forschungsterminen fragt, ob sie die Zeit, die wir mit Lara verbringen, zum Kochen oder Putzen verwenden könne, willigen wir ein. ${ }^{13}$ Einmal erledigt sie einen Behördentermin, zu dem sie Lara ungern mitnehmen möchte. Die Forschungszeiten mit Lara alleine setzen eine stärkere Vertrauensbasis voraus, die mit ihr nach und nach aufgebaut wird. Anhand unserer späteren Feldprotokolle zeigt sich Laras Entwicklung auch im Umgang mit uns Forscherinnen:

Ich krame in meinem Materialienordner herum, und Lara fragt erstaunt: ,,Uh, wat das?", als ich die Sticker heraushole. Ich zeige ihr unterschiedliche Motive, und sie ruft: „Oh!“. Sie darf sich zwei Sticker aussuchen. Sie wählt eine Ente aus und sagt: ,"Quak, quak, quak", als Sarah es ihr vormacht. [...] Als ich ihr den roten Smiley (mit heruntergezogenen Mundwinkeln) zeige, sagt Lara: „Oh no!" [...]. Bei dem grünen Smiley (mit hochgezogenen Mundwinkeln), meint sie „,Hooray! “ [...] Später zeigt sie Richtung Spüle an der anderen Seite des Raums und fragt wieder: ,Wat das?" Ich gehe darauf ein und frage erstaunt, was sie meine. Ich stehe auf und lasse mich von Lara führen. Sie zeigt auf eine Ablage, auf der Kaffee und andere Dinge stehen. Ich hebe sie vorsichtig hoch, und sie zeigt mir eine blaue Keramikschüssel. Wir schauen hinein und entdecken Kekse und Zucker (Feldbesuch 9, GU 1, HMT, 10.8.2016).

Im Verlauf der Forschung werden auch Forschungsmaterialien für Lara interessant. Ihr verbales Nachahmen und ihre Frage „Wat das?" stellen erweiterte Möglichkeiten ihrer Artikulation mit uns Forscherinnen dar. Dabei spricht sie Deutsch und Englisch und findet ihre eigenen Begriffe, um die von SmileyBildern dargestellten Emotionen in ihre Sprache zu übersetzen. Unsere Interaktionen beziehen sich aufeinander und die Beziehung ist weniger distanziert als zu Beginn, vor allem beim Blick- und Körperkontakt. Auch erschließt sich Lara neue Spielmöglichkeiten durch uns Forscherinnen und einen größeren Erkundungsradius. Das individuelle Spiel wird zum gemeinsamen Entdecken. Ihre Lernzuwächse, die wir in ihrer zeitlichen Dimension im Feld als prägnant wahrnehmen, gehen einher mit sich erweiternden Aneignungs- und Gestaltungsmöglichkeiten, auch in sozialer Hinsicht: In der Kita beteiligt sich Lara an einem Waldspaziergang und sie hat eine Freundin gefunden, was die Mutter kaum glauben kann.

13 Wir sind uns bewusst, dass wir in unserer Rolle als Forscherinnen mitunter auch als Ressource, als Kinderbetreuerin, Streitschlichterin, Erziehungsberaterin, Vermittlerin für psychosoziale Hilfen, Spielpartnerin und Zuhörerin multifunktional wahrgenommen und genutzt wurden. 
Dann, eines Abends erreicht uns eine Nachricht von Sandra:

,Wir müssen morgen früh in die Ausländerbehörde. Wir haben eine Ablehnung für das zweite Asyl bekommen und müssen wahrscheinlich jetzt am Samstag verreisen (-) Es droht uns sonst eine zwanghafte Abschiebung. Morgen gehe ich los, damit wir unsere Pässe zurückbekommen und abreisen. [...] Ich möchte mich nochmal bedanken für alles, was ihr für Lara gemacht habt" (Protokoll 10, GU 1, SF, 30.8.2016).

Wir sind geschockt und verabreden uns für den nächsten Abend, um uns von der Familie zu verabschieden. Die Mutter beschreibt ihre Erfahrungen, die sie in Deutschland machen durfte als ,groß“ und stellt diese in Kontrast zu dem Hass, dem sie vorher begegnet war. Sie erzählt, dass in Laras Kita ein Spendenaufruf gestartet und eine Abschiedsfeier für Lara veranstaltet worden sei. Die Kitaleitung setzt sich mit der Härtefallkommission auseinander, die Ärztin hat eine Sachbearbeiterin angerufen. Das Reiseverbot für Lara gelte aber nicht, da sie aus einem sogenannten sicheren Herkunftsland komme. Zur Situation in Bosnien äußert sich die Mutter besorgt. Sie sagt, dass Lara Bosnien nicht kenne, und meint kopfschüttelnd:

„Ach, dort weiß man doch gar nicht, wie man mit Autismus ${ }^{14}$ umgeht, das ist doch auch hier noch kaum erforscht!" Die Betreuung bleibe dann an ihr hängen, aber sie [die Mutter] habe hier eine Menge gelernt. Sie wirkt zuversichtlich. Sie lächelt uns an und umarmt uns beide gleichzeitig (Feldbesuch 11, GU 1, SF, 31.8.2016).

So kommt es zu einem zweiten plötzlichen Forschungs- und Beziehungsabbruch. Durch die Spielbeobachtungen und Interaktionen mit Lara und die Erzählungen ihrer Mutter gewinnen wir jedoch einen kleinen Einblick, wie sich Laras Alltag in der Gemeinschaftsunterkunft gestaltet. Die Handlungsbegrenzungen werden deutlich von ihren Bedürfnissen nach Routine auch in ihrem Bewegungsradius gesetzt. Hinsichtlich ihres sozialen Umfelds erwähnt ihre Mutter uns gegenüber vor allem den Einsatz der Integrationskita-Mitarbeiter*innen und der Ärzt*innen, weniger die Kontakte zu Bewohner*innen oder Mitarbeiter*innen der Unterkunft. Nur wenig Austausch konnte direkt beobachtet werden. Von ihren Geschwistern lebt Lara getrennt. In so konstant wie möglich geschaffenen Alltagsstrukturen und dem relativ stabilen Umfeld von Gemeinschaftsunterkunft, Integrationskita und ärztlicher Versorgung lernt sie dazu, macht für uns erstaunliche Fortschritte in ihrer Entwicklung und erweitert ihren eigenen Handlungsraum.

14 Wir haben uns bewusst dafür entschieden, Laras Diagnose des frühkindlichen Autismus nicht unseren Beobachtungen und Interpretationen voranzustellen, um $\mathrm{zu}$ vermeiden, dass ihre beschriebenen Handlungsweisen auf diese Diagnose zurückgeführt werden. Gleichzeitig bräuchte es eine andere Expertise, um ihr Verhalten psychologisch zu kontextualisieren. 
Nach der Abreise erreicht uns eine E-Mail:

„Hallo Mai@:). Tut mir leid, dass ich mich so spät melde. Es sind einfach zu viele Aufgaben. Die 4 Mädels gehen in die Schule, und Lara geht es nicht besonders gut. Die Veränderung hat bewirkt, dass sie sich in sich zurückgezogen hat. Sie will nicht aus dem Haus. Sie ist verwirrt. Hier bekommt sie keine Förderung und kein Recht. Solche Kinder sind auf sich selbst und ihre Eltern angewiesen. Ich bemühe mich, ihre Tage zu erleichtern. Ihr Papa arbeitet jeden Tag. Das Leben hier ist sehr schwer, und alles ist extrem teuer: Lebensmittel und Miete - irgendwie dreht sich alles ums Überleben. Es wird schon ... wir schaffen das (-) Gott, ich komme mir vor wie Frau Merkel mit ihrem Satz Viele Grüße!" (Protokoll 12, GU 1, SF, 21.9.2016)

\subsection{Spiderman: Der Rebell im Zwischenraum}

Spiderman ${ }^{15}$ ist sechs Jahre alt und bewohnt mit seiner achtjährigen Schwester Jule, der vier Monate alten Jana, den Eltern Omar und Nada zwei Zimmer mit Bad (ca. 35 Quadratmeter) in einer großen Gemeinschaftsunterkunft im Randgebiet von Berlin. Die Familie kommt aus dem Irak und ist seit einem Jahr in Deutschland. Ihr Asylantrag wurde abgelehnt, was die Eltern auf einen Übersetzungsfehler bei der Anhörung zurückführen. Sie sprechen Arabisch, der Vater etwas Englisch und Deutsch, Jule und Spiderman Deutsch und Arabisch. Jule geht in die zweite Klasse, Spiderman in die Kita. $\mathrm{Zu}$ Beginn hatten wir Schwierigkeiten, mit Spiderman in verbale Interaktion zu treten. Er drückt sich lieber mittels Bewegung und in Aktion aus:

Als ich Spiderman frage, wo er sich noch so im Haus aufhalte, rennt er in das Treppenhaus, springt seitlich auf das Geländer und rutscht sehr schnell herunter, was mich beeindruckt. Im Erdgeschoss angekommen, macht er vor der Tür der hausinternen Kinderbetreuung halt. Die Betreuungszeit (Montag bis Freitag von 14 bis 17.30 Uhr) ist bereits vorüber, viele Kinder stehen aber noch vor der Tür. Es ist sehr laut. [...] Ich frage Spiderman, ob er oft in der Kinderbetreuung sei, und er antwortet: "Ich geh nicht mehr da hin. Das ist nur [für] Babys. Da mag ich's nicht. " [...] Wir gehen weiter zum Gemeinschaftsraum links neben der Kinderbetreuung, und er sagt: „Ich darf nicht in den Raum rein." Der Gemeinschaftsraum ist nur für Kinder ab zwölf Jahren zugänglich, so steht es auf einem Schild an der Tür. [...] Im Erdgeschoss gibt es mehrere Kursräume und viele Abzweigungen. Spiderman nähert sich dem Gemeinschaftsraum von einer anderen Richtung, steht herum, lacht und zeigt hinein.

15 So der selbstgewählte Name des Jungen. Auch die Namen seiner Eltern und seiner Schwester Jule sind von diesen selbst gewählt. Der Name der jüngeren Schwester Jana wurde von Jule ausgesucht. 
Er betritt den Raum, singt laut und etwas schadenfroh: „Nenenene, nenenene!", und streckt den wenigen anwesenden Erwachsenen seine Zunge heraus. Dann rennt er wieder zurück zu mir in den Flur und weiter Richtung Kursräume. [...] Er stoppt vor einem der Zimmer ab und sagt: ,,Von dem Zimmer hab ich meine Flöte genommen. "Auf einem Zettel neben der verschlossenen Tür steht: „,Blockflötenunterricht fällt heute aus “. Spiderman rennt weiter, das Treppenhaus herunter, öffnet eine Stahltür und zeigt mir das leere Untergeschoss. [...] Er ruft: „Hey! Schau mal! “, nimmt sich einen Roller und fährt damit umher (Feldbesuch 7, GU 2, SF/HMT, 2.2.2017).

Die Hausbegehung mit Spiderman zeigt, wie er die für ihn relevanten physischen Räumlichkeiten der Unterkunft erfährt, sich gegenüber Begrenzungen positioniert, selbst auf soziale Zugehörigkeiten anhand von Alterskategorien Bezug nimmt und sich in Transiträumen wiederfindet. Die vielen Flure und das Treppenhaus nutzt er, um seine Schnelligkeit und Fertigkeit im Rennen und Rutschen auszuleben. Er eignet sich diese Zwischenräume aktiv an und navigiert geschickt darin, findet alternative Wege zum gleichen Ziel. Er führt die Forscherin als Erstes zur bereits geschlossenen Kinderbetreuung. Der Flur wird von den anwesenden Kindern nach Ende der Betreuungszeit als Aufenthaltsort genutzt. Mit den Kindern tritt Spiderman nicht in Interaktion. Er grenzt sich von der Kinderbetreuung ab mit der Begründung, dass dies nur für Babys sei und er sie deshalb nicht möge. Er impliziert, dass er schon zu alt für diesen Raum sei, obwohl die Kinderbetreuung für Kinder bis zwölf Jahre vorgesehen ist.

Der nächste bedeutsame Raum ist der Gemeinschaftsraum, der Kinder bis zwölf Jahre ausschließt. Für diesen Ort ist er nicht alt genug. Er weiß, dass dieser Raum für ihn nicht zugänglich ist, obwohl er das Schild mit den entsprechenden Vorschriften nicht lesen kann. Durch einen anderen Eingang verschafft er sich Zutritt und inszeniert diese Grenzüberschreitung verbal und nonverbal.

Unter den vielen verschlossenen Kursräumen ist einer für Spiderman bedeutsam. Er assoziiert spontan, dass er aus diesem Raum eine Flöte mitgenommen hat. Über den Unterricht selbst macht er keine Aussage. An diesem Tag findet kein Kurs statt, somit bleibt der Raum in seiner Funktion ebenfalls nicht zugänglich. Als letzte Raumstation betritt Spiderman das Untergeschoss durch eine massive Stahltür. Er wird sofort aktiv, nimmt sich einen Roller, fährt los und fordert die Forscherin auf, ihm zuzuschauen.

Deutlich wird an der Bewegungsraumexploration innerhalb der Unterkunft, dass Spiderman sich viel in Zwischenräumen bewegt, sie eigensinnig durch seine Bewegung (um-)funktionalisiert (beispielsweise spielt er im Treppenhaus Flöte, fährt im Flur Fahrrad und Roller im Untergeschoss) und die „eigentlichen Räume“ ihm aus unterschiedlichen Gründen verschlossen bleiben. 
Die Einschränkungen im Bewegungs- und Handlungsraum, die das Leben in der Gemeinschaftsunterkunft mit sich bringen, beschäftigen Spiderman, wie in einem Gespräch an selben Tag deutlich wird:

Ich frage: ,Wie findet ihr das hier, dieses Zuhause? Ist das gut oder ist das doof? "Spiderman: „Doof. Ich will ein echtes Haus. “ [...] Ich hake nach: ,, Warum findest du es denn hier doof?" Er sagt: ,Weil alle sagen: Das darf ich nicht machen, das darf ich nicht machen! [...] Die Security sagt, ich darf keine Musik machen. Die Security sagt, ich darf nicht mit meiner Flöte spielen." Seine Schwester Jule erklärt: „,Es gibt eine Regel, [...] wenn die Kinder Flöte spielen, [...] weil das zu laut ist, weil manche wollen schlafen. " Spiderman fügt hinzu: ,Aber warum? Ich will in der Nacht aufwachen. Ich will in der Nacht nicht schlafen! " (Feldbesuch 7, GU 2, SF/HMT, 2.2.2017)

Spiderman rahmt die Unterkunft negativ: Es ist kein „echtes Haus“, weil es Vorschriften gibt, die Spiderman in seinen Handlungen einschränken. Er betont diesen Umstand, in dem alle ihm vorschreiben, was er nicht tun soll. Es handelt sich um Imperative, die ihm ausschließlich die Grenzen seines Handelns aufzeigen anstelle von Möglichkeiten, um seine Handlungsfähigkeit auszuleben. So wird sein Flötenspiel in der Unterkunft als Lärmbelästigung definiert und ist auch (bzw. gerade) in den Fluren verboten. Er verweist auf die „Security“, den Sicherheitsdienst, der im Unterschied zu einem ,echten“ Zuhause die Einhaltung der Hausordnung in der Unterkunft sicherstellt und gegebenenfalls sanktioniert. Das Leben in der hellhörigen Gemeinschaftsunterkunft verlangt von Spiderman, sich nach den (Schlaf-)Bedürfnissen der Bewohner*innen und der Hausordnung der Unterkunft zu richten. Spiderman kann diese Regeln nicht nachvollziehen. Er stellt dem Bedürfnis von Ruhe und Schlaf sein eigenes Bedürfnis nach Aktivität und Wachsein entgegen.

Regelungen wie diese stellen nur ein Beispiel von vielen dar: Kinder dürfen sich nur zu bestimmten Zeiten im Flur aufhalten, dürfen den Fahrstuhl nicht benutzen, dürfen bestimmte Räume nur zu bestimmten Zeiten besuchen oder sie aufgrund ihres Alters gar nicht betreten. Kinder unter sechzehn Jahren bekommen auch keinen eigenen Schlüssel für die Bewohner*innenzimmer ausgehändigt. Ab zwölf Jahren dürfen sie den Schlüssel ihrer Eltern mitbenutzen. Als Spiderman an einem anderen Tag die Kinderbetreuung verlässt, rennt er Richtung Privatraum der Familie:

Wir folgen ihm und als wir auf der ersten Etage ankommen, hämmert er bereits mit seiner Faust gegen die Tür und tritt wütend auf sie ein. [...] Er ist sehr aufgebracht darüber, dass er nicht herein kann. Er lässt nicht von der Tür ab und tritt mit voller Wucht mehrmals auf sie ein. Dann rennt er plötzlich zu einem Abstellraum und schließt die Tür hinter sich. Ich klopfe vorsichtig an. Er hat drinnen sein kleines, gelbes Fahrrad in der Hand und sagt: „Das ist meins “ (Feldbesuch 9, GU 2, SF/HMT, 14.2.2017). 
Als Spiderman merkt, dass er vor verschlossener Tür steht, wird er wütend. Nach einigen Versuchen, die Tür mit bloßer Kraft einzutreten, flüchtet er sich in eine Abstellkammer auf dem Flur der Etage. Dort schließt er die Tür hinter sich und schafft sich so eine Art vorübergehenden Privatraum. Als wir nach ihm schauen, finden wir ihn neben vielen Fahrrädern und Rollern mit seinem eigenen Fahrrad vor, das er mit den Worten „Das ist meins“ festhält. Es ist kein Zufall, dass Spiderman bei all den Reglementierungen und verschlossenen Türen zu dem rennt, was ihm gehört, ihm zugänglich ist und seine Mobilität symbolisiert. Wenig später kommt seine Mutter wieder und schließt die Wohnungstür auf.

Spiderman kennt viele Kinder in der Unterkunft, bezeichnet sie aber als „Bekanntschaften“, nicht als Freunde. Die Freundschaft zu seiner Schwester Jule wird in Streitereien häufig verhandelt. Seine Mutter Nada gibt an, dass sie seit der Geburt von Jana weniger Zeit für Spiderman habe, gestaltet aber häufig das Kinderzimmer mit ihm um. Zu seinem Vater sucht Spiderman Körperkontakt und sie spielen Handyspiele miteinander. Der Vater gibt jedoch an, durch vielfältige Belastungen nicht ausreichend für seine Kinder da sein zu können. Die Eltern selbst haben ein soziales Netzwerk von Verwandten, Freunden und ehrenamtlichen Helfer*innen, die Spiderman in der Forschung nicht erwähnt. Wenn er nicht in der Kita ist, verbringt er die meiste Zeit im Privatraum der Familie. Seine Mutter berichtet übersetzt von unserer Sprachmittlerin:

„Manchmal geht er [Spiderman] in die Kinderbetreuung, aber in der Regel streitet er und kommt dann zurück. [...] Er wollte schon immer in die Schule gehen, aber seine Sprachkenntnisse reichen noch nicht aus. [...] In der Kita im Irak hat er nur das arabische Alphabet gelernt, aber nicht mehr. [...] Manchmal spricht er mit uns auf Deutsch und wir verstehen ihn nicht. Wenn wir dann nachfragen, sagt er: ,Ich weiß nicht, was das auf Arabisch heißt " " (Feldbesuch 7, GU 2, SF/HMT, 2.2.2017).

Die Mutter erzählt, dass Spiderman in die Schule gehen wolle, was auch an sein Bedürfnis anschließt, älter zu sein, als er ist. Seine Deutschkenntnisse würden allerdings als nicht ausreichend für seine Einschulung eingeschätzt. Nicht nur für den Zugang zur Schule und die Beziehung zu Gleichaltrigen, auch für die familiäre Interaktion ist Sprache sehr wichtig. In der Kita im Irak hat er das arabische Alphabet gelernt; dies wird in Deutschland jedoch nicht weiter gefördert, und die arabische Sprache geht langsam verloren. Von seinen Eltern wird er teilweise nicht verstanden, wenn er auf Deutsch mit ihnen spricht, und Spiderman gibt auch uns gegenüber an, die Worte auf Arabisch nicht mehr zu kennen. Der Beziehungsraum ist durch eine gewisse Sprachlosigkeit und Kommunikationsbarrieren gekennzeichnet. Spiderman ist auch hier in einem kommunikativen Zwischenraum gefangen und durch die unzureichenden Kenntnisse beider Sprachen in seiner Handlungsfähigkeit in sozialen Beziehungen, vor allem in seiner Familie, eingeschränkt. Auf kreative und 
fantasievolle Art und Weise schafft er sich jedoch einen anderen Spielraum in der Interaktion mit seinen Kuscheltieren:

Auf den Spinten und auf dem Bett liegen viele Kuscheltiere, die er mir vorstellt. [...] Auf einmal wird er stiller und meint: ,Ich hab sie mitgenommen und dann ist sie weggefallen ...", er schaut zu Boden und ergänzt traurig: „, Mein süßer Affe ... ". Zu den anderen Tieren meint er: „Ich mag die alle! $D u, d u, d u$... die sind alle so ... schön. " Dann macht er silbenähnliche Geräusche in einem eher kindlichen Ton und kuschelt mit den Tieren. [...] Er meint: „Die sind hergezaubert [...]. Ich weiß was, die Kuscheltiere ... sie reden in der Nacht. Sie haben Angst ... jaaa ... “ (Feldbesuch 8, GU 2, HMT/SF, 11.2.2017).

Im Kinderzimmer, das er sich mit seiner Schwester Jule teilt, beobachten wir an Spiderman im Umgang mit seiner Kuscheltiersammlung eine liebevolle und einfühlsame Seite. Er bedauert den Verlust eines Kuscheltieres, das ihm auf der Flucht aus dem Irak abhandenkam. Er verleiht seinen Kuscheltieren etwas Magisches, findet sie alle schön, spricht zu ihnen und gibt an, dass sie auch zu ihm sprächen. Sprachliche Unzulänglichkeiten werden in dieser engen Beziehung unwichtig. Er schafft sich kreativ und selbstständig eine eigene imaginierte Welt, in der er positive Resonanz erfährt. Er ergänzt, dass die Tiere Angst in der Nacht hätten. Von der Mutter wissen wir, dass er nachts häufig aufwacht, einnässt und ängstlich ist. Die Mutter beschreibt ihn als traumatisiert. Sie erklärt sein Verhalten mit Gewalterfahrungen im Irak, wo er aus Angst die Wohnung nicht mehr verlassen konnte. Sie erzählt von Explosionen, Bombardements und der Auseinandersetzung mit Tod und Verlust. Später äußert sich Spiderman zur Situation im Irak und zum Bleibestatus der Familie:

„Ich liebe Deutschland. Irak ... bum bum“, und macht ein Gewehr gestisch nach. Er spricht weiter auf Arabisch (was uns die Sprachmittlerin übersetzt): „,Wenn wir gehen müssen, rausgeschoben sind, werde ich sie töten - die Polizisten ... wenn sie uns in Irak schicken. "Dann merkt er an, dass er allerdings im Irak auch seinen Affen wiederfinden könnte. Der Vater meint daraufhin: „Wir hatten ein schönes Leben im Irak. Wir waren reich und hatten ein Auto ... “, woraufhin Spiderman einwirft: „Hier gibt es U-Bahn und Straßenbahn!', was alle kurz zum Lachen bringt. [...] Ich setze mich zu ihm hin, und er sagt ganz leise: „,Deutschland ist besser als Irak. Im Irak kann ich gar nichts machen. [...] Weißt du? Papa lügt. Was Papa sagt: Du musst sofort wieder gehen in Irak, aber ich will nicht gehen. Ich geh nicht. Ich darf nicht gehen " (Feldbesuch 8, GU 2, HMT/SF, 11.2.2017).

Spiderman erinnert sich nicht nur an die Zustände im Irak und an die Flucht, sondern setzt sich gedanklich auch mit dem Thema Abschiebung auseinander. Im Kontrast zum Herkunftsland, das er mit Krieg verbindet, empfindet Spiderman Liebe zum Aufnahmeland. Die Bedeutung von seinem verlorenen Kuscheltier, das er noch immer betrauert, wird ebenfalls kurz deutlich. Als der Vater ergänzt, dass sie im Irak ein schönes Leben gehabt hätten, und den 
Statusverlust der Familie beklagt, widerspricht Spiderman ihm und macht ihn auf die öffentlichen Verkehrsmittel aufmerksam, die die Mobilität der Familie ebenfalls sicherten. Er will seinem Vater nicht glauben, dass sie in den Irak zurückkehren müssen. Das Spannungsverhältnis in Spidermans von Abschiebung bedrohter Lebenssituation wird deutlich, wenn der Satz „Ich darf nicht gehen“ mit seiner Aussage „Weil alle sagen, das darf ich nicht machen“ zu Beginn dieses Abschnitts kontrastiert wird. Die vielen Reglementierungen der Unterkunft, die Spiderman in seinem Handlungsspielraum einschränken, werden von allen mitgetragen (,alle sagen das darf ich nicht machen“), wobei die strikte Ablehnung der Abschiebung lediglich von Spiderman selbst formuliert wird („Ich darf nicht gehen“). Im Fall der Fälle würde Spiderman die Polizisten töten, die ähnlich wie der Sicherheitsdienst der Unterkunft für „Ordnung“ sorgen. Hier wird deutlich, dass Spiderman sein Recht auf Aufenthalt in Deutschland einfordert, was seinen Anspruch auf Selbstbestimmung markiert und gleichzeitig die Unsichtbarkeit bzw. Unhörbarkeit dieser Forderung unterstreicht, da sie kein institutionelles Gehör findet. Dieser Umstand markiert die Widersprüche in Spidermans Lebensverhältnissen: Es gibt keine verlässliche Instanz, die ihn vor der Abschiebung in den Irak schützt und gleichzeitig existieren viele Reglementierungen, die ihn in seinen Möglichkeiten, mitzubestimmen und zu handeln, beschneiden.

Spidermans Handlungsspielraum wird durch die Begrenzungen der Unterkunft gesetzt und teilweise von ihm durchbrochen. Er erschließt sich Räume (vor allem nicht vordefinierte Zwischenräume) im Modus der „raumgreifenden“ Bewegung. Viele Räumlichkeiten sind in ihrer Funktionalität nicht auf die Bedürfnisse von Spiderman ausgerichtet, Ausnahme bilden die Privaträume der Familie, die jedoch, wie andere Räume in der Unterkunft, für ihn nicht konstant zugänglich sind. Auch sein Beziehungsraum kann als sozialer Zwischenraum und Aushandlungszone von Zugehörigkeiten in der Interaktion mit Gleichaltrigen, Familienmitgliedern und seiner Positionierung in der deutschen Gesellschaft verstanden werden. Die Erschließung neuer Handlungszusammenhänge wird durch Sprachbarrieren, Abgrenzungen und Reglementierungen erschwert. Die ungewisse Aufenthaltsperspektive der Familie beunruhigt ihn, beschäftigt seine Eltern und schafft auf vielfältige Weise eine belastende Familiendynamik. Sein Vater drückt dies mit den Worten aus: „Ohne Stabilität kann man keine Wünsche haben“. Spidermans Gesundheitszustand wird von seinen Eltern als beeinträchtigt und von Mitarbeiter*innen der Unterkunft als psychosozial unzureichend begleitet eingeschätzt. Als Ausdruck seiner Handlungsfähigkeit erfindet er sich im Spiel mit Kuscheltieren seinen eigenen strukturierten und fantasievollen Spielraum.

Nach dem Ende unserer Forschungszeit erfahren wir, dass auch der von der Familie eingelegte Widerspruch auf die Ablehnung ihres Asylantrags abgewiesen wurde. Die Familie lebt seitdem in Duldung in der Gemeinschaftsunterkunft. Beide Eltern suchen nach einer Ausbildungsmöglichkeit, um ihren Auf- 
Handlungs-Spiel-Räume von Kindern in Gemeinschaftsunterkünften 129

enthaltsstatus zu verbessern. Spiderman geht in die Schule und besucht einen Karatekurs.

\section{Handlungs-Spiel-Räume: Zusammenführende Diskussion}

In diesem Beitrag sind wir anhand von drei Fallstudien der Frage nachgegangen, wie Kinder ihren Alltag in einem von asylpolitischen Entscheidungen geprägten Kontext (zwischen Sandkasten als junge Kinder und Abschiebung als „nichtbleibeberechtigte“ Geflüchtete) in Gemeinschaftsunterkünften leben. Als Analyseraster dienten uns hierbei ihre ineinander übergreifenden Erfahrungen, Aneignungs- und Gestaltungspraxen von und durch physischen/materiellen Raum, Beziehungsraum und Handlungsraum.

Die räumliche Beschaffenheit von Unterkünften, in denen geflüchtete Menschen untergebracht werden, sind unterschiedlich, doch handelt es sich in den meisten Fällen um „Sammelunterkünfte“, in denen viele Menschen auf beengtem Raum wohnen und die Möglichkeiten des Rückzugs und der Schutz der Privatsphäre gering sind (vgl. Lewek \& Naber 2017: 22 f.). Institutionelle Normen und Regeln beschneiden den Privatraum der Familien. Die Unterkünfte sind in erster Linie - wenn überhaupt - eher als Unterbringungsorte für Erwachsene konzipiert, was sich in einer oftmals nicht kindgerechten Raumplanung und den kindlichen Bedarfen und Bedürfnissen nicht entsprechenden „Verregelung" niederschlägt, wie in unseren Fallstudien beschrieben. Sie können daher als ,ungeeignete Aufenthaltsorte für Kinder" und ihre Familien (World Vision \& Hoffnungsträger Stiftung 2016: 49) betrachtet werden. Wir möchten sie jedoch nicht, wie in der Literatur zuweilen verhandelt, als ,totale Institutionen“ nach Goffman (1973), „Disziplinaranstalten“ à la Foucault (1994), oder Agamben'sche „Lager“" (2002), in denen der Ausnahmezustand die Regel ist, begreifen (vgl. Schäfer 2015: 17). Ein solcher Fokus auf die Charakterisierung ordnender Strukturen würde uns als Interpretationsfolie den Blick auf die vielseitigen, alltäglichen, räumlichen Erfahrungen, Praktiken und Taktiken von Kindern verengen und der Heterogenität und Widersprüchlichkeit der von ihnen erlebten Unterbringungssituationen nicht gerecht werden (vgl. ebd.). Die Kinder in unseren Fallstudien eignen sich Räumlichkeiten unterschiedlich an, sie schaffen sich Räume in ihrem Tun, geben ihnen Bedeutung, lassen sie genutzt oder ungenutzt und verräumlichen so handelnd ihre Umgebung (De Certeau 1988: 345 ff.). Sie setzen sich mit den Unterkunftsverhältnissen, dem Zugang zu Räumlichkeiten, den Bewegungsmöglichkeiten, den sanitären Bedingungen, den Regeln und ihrer Durchsetzung auseinander. Dabei haben sie auf die Wohnraumzuweisung keinen Einfluss. Die Trennung 
von öffentlichem und privatem Raum ist fließend und wird herausgefordert (vgl. White 2012: 321 f.). Die „Zwangsgemeinschaft" (World Vision \& Hoffnungsträger Stiftung 2016: 49), in der sie ihren Alltag je nach Ausstattung mit Gemeinschaftsküchen, -duschen, -waschräumen, Rückzugs-, Spiel- und Gemeinschaftsräumen strukturieren, ist ihnen mehr oder weniger bewusst. Zum Teil wird die Unterbringung als nicht ,echt" betrachtet und als diskriminierend erlebt (vgl. Berthold 2014: 40). Eigene Räume in und außerhalb der Unterkunft stellen eine wichtige Ressource dar, die unterschiedlich erschlossen und genutzt wird (vgl. Archambault 2012: 40 ff.).

In Bezug auf den Beziehungsraum ist die soziale Zugehörigkeit zur Familie ein wichtiger, verlässlicher Bezugspunkt für Kinder (vgl. World Vision \& Hoffnungsträger Stiftung: $44 \mathrm{ff}$.). Zu differenzieren sind innerfamiliär unterschiedliche Familienkulturen, Rollenverteilungen, die Größe der Familie, ihre spezifischen Lebenslagen und innerfamilialen Dynamiken, die sich belastend oder unterstützend auf den Alltag der Kinder auswirken. Trennungen und Verluste von Familienmitgliedern, Freund*innen und Verwandten, wie auch die Kontaktpflege über das Internet und soziale Medien sind bedeutsam für Kinder und spiegeln ihr soziales Netzwerk jenseits nationalstaatlicher Bezüge wider. Gleichzeitig werden neue Beziehungen aufgebaut und Netzwerke erweitert, welche für die sozialen Zugehörigkeiten der Kinder wichtig sind (vgl. Archambault 2012: 45). Aus unseren Fallstudien lässt sich allerdings herauslesen, dass ein großer Pool an Peerkontakten nicht automatisch zu Freundschaften führt, sondern dass diese, wie auch altersbestimmte und sprachbasierte Zugehörigkeiten, Gegenstand beständiger Aushandlungs- und Abgrenzungsprozesse sind. Unterstützungssysteme durch Kinderbetreuer*innen, Mitarbeiter*innen der Unterkunft, weitere Professionelle, Ehrenamtliche und andere (geflüchtete) Familien sind ebenso zentral. Der Erhalt und Abbruch von Beziehungen zu Menschen aus diversen Sprachräumen hat Konsequenzen für die mehrsprachigen Fähigkeiten der Kinder. Ihre transnationalen Bezüge stellen eine Erweiterung für tradiert nationalstaatlich geprägte Kindheiten dar und verweisen auf einen „grenzüberschreitenden Aktionsradius“ (Himmelbach \& Schröer 2014: 493 ) von Kindern und ihren Familien.

Den Handlungsraum bzw. die Agency der Kinder betrachten wir als Fähigkeit und Möglichkeit, als Subjekte tätig zu sein und innerhalb sozialer, räumlicher und zeitlicher Bezüge zu handeln (James \& Adrian 2008: 9). Dabei erkennen wir an, dass Kinder ebenfalls auf diese lebensweltlichen Strukturen einwirken. Sie fordern Zeit ein, zweckentfremden Räume nach ihren Interessen und wirken auf soziale Beziehungen ein. Sie eignen sich die Welt an, sind soziale Akteur*innen und entwickeln eigene Deutungs- und Handlungsmuster (Hungerland \& Kelle 2014: 228). Wir betrachten Agency nicht nur als persönliche Eigenschaft, sondern als sozial bedingt und verwoben mit den Lebensverhältnissen, in denen Kinder aufwachsen. Diese sind Bedingung und Produkt ihres Handlungsvermögens und relational zu betrachten (vgl. Eßer 2014: 
236 f.). Wie unterschiedlich spezifische Lebensverhältnisse von Kindern antizipiert, interpretiert und gelebt werden, haben wir anhand unserer drei Fallstudien herausgearbeitet. Zwar ist den Kindern gemein, dass sie mit unsicherem Aufenthaltsstatus in Gemeinschaftsunterkünften in Berlin leben, doch wird ihre Diversität hinsichtlich ihrer Bleibemöglichkeit, des Asylrechtsgrunds, der Sprachen, der familiären Dynamik, Familienform, Unterbringungssituation, Gesundheit, der sozialen Herkunft, Alltagsgestaltung und der Persönlichkeit jedes Kindes deutlich. Diese Diversität spiegelt sich auch in den Erfahrungen der Kinder von Bewegungs-, Beziehungs- und Handlungsräumen in den jeweiligen Begrenzungen und Möglichkeiten wider: Elsa - die selbstbestimmte Macherin - schafft sich Beziehungen und Bewegungsräume (,doing relations“/,space“), verteidigt diese aktiv, fordert ihren Raum und ihre Zeit für Spiel ebenso wie für Rückzug. Lara - die lernende Perfektionistin - schafft sich Ordnung in Beziehungen, Räumen und in ihren Handlungen; sie fordert Routinen und Gewissheiten ein und nutzt diese, um ihre Spielräume zu erweitern. Spiderman - der Rebell im Zwischenraum - setzt sich mit den Beschneidungen seiner Lebensverhältnisse im Bewegungs-, Beziehungs- und Handlungsraum auseinander. Er stellt sie in Frage, positioniert sich zu ihnen und fordert Veränderungen (ein „,echtes Haus“, eine Bleibeperspektive).

Ihre Erfahrungen stehen jedoch nicht nur für jedes Kind an sich, sondern verdeutlichen die aktive Auseinandersetzung mit ihrem Lebenskontext in den Begrenzungen und Möglichkeiten, die sie erfahren (vgl. White 2012: 314). Diese Kinder sind offensichtlich mehr als das „Produkt von Sozialisationsprozessen“, mehr als nur „Anhängsel“ ihrer Familie (Liebel 2017: 40).

Die Ambivalenz der politischen Identität als „Kinder“ und „Geflüchtete“ behandelt geflüchtete Kinder in Deutschland jedoch nach dem Asylgesetz und missachtet dabei grundlegende Rechte, wie sie in der UN-Kinderrechtskonvention formuliert sind (Pro Asyl 2011; siehe den Beitrag von Wihstutz „Mittendrin und außen vor"). Der limitierte Zugang zu gesellschaftlichen Ressourcen und Möglichkeiten sowie die Begrenztheit von Entscheidungsspielräumen und Positionen verdeutlichen ihre fremdbestimmten Lebensverhältnisse als gesellschaftsstrukturelle Ausgrenzung (Eisenhuth 2015: 29; Liebel 2017: 32). Asylpolitische Diskurse konstituieren so die Lebenslagen der Familien in ihrer praktischen Konsequenz als Ausdruck geopolitischer Strategien und globaler Machtverhältnisse. Sie bilden die Grundlage der erlebten Handlungsspielräume, die je Familie und Kind unterschiedlich genutzt und wahrgenommen werden - und in denen sich jedes Kind mit seinem lebensweltlichen Eigensinn in alltäglichen, räumlichen Praktiken und Taktiken einschreibt. 


\section{Schlussfolgerungen und Forderungen}

Aus diesem Beitrag ergeben sich einige Schlussfolgerungen: Auf konzeptueller Ebene zeigt diese Studie, wie fruchtbar räumliche Analysekategorien die Lebenswirklichkeiten von jungen Kindern in „Sammelunterkünften“ abbilden können und wie eng - und auf unterschiedliche Art und Weise - physische Raumerfahrungen mit Beziehungsräumen und Handlungsräumen verwoben sind. Diese Erkenntnisse können auch für die Felder der Integration, Inklusion, Lebensweltorientierung und Sozialraumvernetzung wegweisend sein. Die hier dargestellten Erfahrungen, Themen und Handlungen der Kinder sollten außerdem Folgen für die Praxis haben (z. B. in Unterkünften, für Behörden, Organisationen, Ehrenamtliche, Kindertageseinrichtungen): Kinder und Familien sind in die Raumplanung, -gestaltung und -nutzung in den Unterkünften einzubeziehen, wenn sie schon kein „echtes Haus“ bzw. keine „echte Wohnung“ bewohnen können. Ferner müssen Beteiligungs- und Beschwerdemöglichkeiten für Kinder ermöglicht werden (vgl. Save the Children o. J.: 18; Lewek \& Naber 2017: 49 ff.). Die Zusammenarbeit mit geflüchteten Kindern und Familien sollte sich eine differenzierte Betrachtung ihrer Lebensverhältnisse zur Prämisse machen und an die jeweiligen Ressourcen, Fähigkeiten und sozialen, räumlichen wie auch sprachlichen Bezüge anknüpfen, die hier nur anhand eines kleinen Samples aufgezeigt wurden.

Und last, but not least: Auch auf politischer Ebene gilt es, Kinder und ihre Belange ernst zu nehmen und Maßnahmen für ihre rechtliche Gleichbehandlung hinsichtlich ihrer Unterbringung, Gesundheitsversorgung, finanzieller Versorgung, des Rechts auf Familie, des Arbeitszugangs ihrer Eltern und des Zugangs zu Bildung zu schaffen. Anstelle von Sonderstrukturen für asylsuchende Kinder und Familien, vor allem für die mit einer erst geschaffenen ,unsicheren Bleibeperspektive“, muss eine strukturelle Integration als Ermöglichung von Teilhabe politisch gewollt und umgesetzt werden.

\section{Literatur}

Agamben, Giorgio (2002): Homo sacer. Die souveräne Macht und das nackte Leben. Frankfurt am Main: Suhrkamp.

Archambault, Josée (2012): ,It can be good there too': home and continuity in refugee children's narratives of settlement. In: Children's Geographies 10 (1), S. 35-48.

Berthold, Thomas (2014): In erster Linie Kinder. Flüchtlingskinder in Deutschland. Hrsg. von Unicef. Köln: Unicef. 
Bollig, Sabine, Honig, Michael-Sebastian \& Nienhaus, Sylvia (2016): Vielfalt betreuter Kindheiten. Ethnographische Fallstudien zu den Bildungs- und Betreuungsarrangements 2-4jähriger Kinder. Belval: Université de Luxembourg.

Breidenstein, Georg, Hirschauer, Stefan, Kalthoff, Herbert \& Nieswand, Boris (2015): Ethnografie. Die Praxis der Feldforschung. 2. Auflage. Konstanz/München: UTB.

Christensen, Pia \& Prout, Alan (2002): Working With Ethical Symmetry in Social Research with Children. In: Childhood 9 (4), S. 477-497.

De Certeau, Michel (1988): Kunst des Handelns. Berlin: Merve.

Dilger, Hansjörg \& Dohrn, Kristina in Collaboration with International Women Space (Hrsg.) (2016): Living in Refugee Camps in Berlin. Women's Perspectives and Experiences. Berlin: Weißensee.

Eisenhuth, Franziska (2015): Strukturelle Diskriminierung von Kindern mit unsicheren Aufenthaltsstatus. Subjekte der Gerechtigkeit zwischen Fremd- und Selbstpositionierungen. Wiesbaden: Springer VS.

Eßer, Florian (2014): Agency Revisited. Relationale Perspektiven auf Kindheit und die Handlungsfähigkeit von Kindern. In: Zeitschrift für Soziologie der Erziehung der Sozialisation (ZSE) 34 (3), S. 233-246.

Fichtner, Sarah \& Trần, Hoa Mai (2018): Ethische Ambivalenzen in der Forschung mit Kindern in Unterkünften für geflüchtete Menschen. In: Forum Qualitative Sozialforschung/Forum: Qualitative Social Research 19 (3), http://www.qualitative-research.net/index.php/fqs/article/view/3150/4307 (Zugriff 2.10.2018).

Foucault, Michel (1980): Power/Knowledge: Selected Interviews and Other Writings 1972-1977. Hrsg. von Colin Gordon. New York: Pantheon.

Foucault, Michel (1994): Überwachen und Strafen. Die Geburt des Gefängnisses. Frankfurt am Main: Suhrkamp .

Goffman, Erving (1973): Asyle. Über die soziale Situation psychiatrischer Patienten und anderer Insassen. Frankfurt am Main: Suhrkamp.

Hackett, Abigail, Procter, Lisa \& Seymour, Julie (2015): Introduction: Spatial Perspectives and Childhood Studies. In: dies. (Hrsg.): Children's Spatialities: Embodiment, Emotion and Agency. London: Palgrave Macmillan, S. 1-17.

Himmelbach, Nicole \& Schröer, Wolfgang (2014): Die transnationale Kindheit. In: Baader, Meike Sophia, Eßer, Florian \& Schröer, Wolfgang (Hrsg.): Kindheiten in der Moderne. Frankfurt/New York: Campus, S. 492-509.

Hungerland, Beatrice \& Kelle, Helga (2014): Kinder als Akteure - Agency und Kindheit. In: Zeitschrift für Soziologie der Erziehung und Sozialisation (ZSE) 34 (3), S. 227-232.

James, Allison \& Adrian, James (2008): Key Concepts in Childhood Studies. Los Angeles, London, New Delhi, Singapore: Sage.

Kaukko, Mervi, Dunwoodie, Karen \& Riggs, Elisha (2017): Rethinking the Ethical and Methodological Dimensions of research with Refugee Children. In: Zeitschrift für internationale Bildungsforschung und Entwicklungspädagogik (ZEP) 1, S. 16-21. 
Lewek, Mirjam \& Naber, Adam (2017): Kindheit im Wartezustand. Studie zur Situation von Kindern und Jugendlichen in Flüchtlingsunterkünften in Deutschland. Köln: Unicef.

Liebel, Manfred (2017): Postkoloniale Kindheiten. Zwischen Ausgrenzung und Widerstand. Weinheim, Basel: Beltz Juventa.

Pro Asyl (2011): Kinderrechte für Flüchtlingskinder ernst nehmen. Gesetzlicher Änderungsbedarf aufgrund der Rücknahme der Vorbehalte zur UN-Kinderrechtskonvention, https://www.proasyl.de/wp-content/uploads/2015/07/PRO_ ASYL_Kinderrechte_ernst_nehmen.pdf (Zugriff 12.9.2017).

Rasmussen, Kim (2004): Places for Children - Children's Places. In: Childhood 11 (2), S. 155-173.

Save the Children (o. J.): Kinderflüchtlinge brauchen Schutz. Berlin: Save the Children.

Schäfer, Philipp (2015): Das Flüchtlingswohnheim. Raumcharakter und Raumpraxis in der Gemeinschaftsunterkunft. In: sinnprovinz. Kultursoziologische working papers Nr. 7. Universität Leipzig.

Seeberg, Marie Louise, Bagge, Cecilie \& Enger, Truls André (2009): No place. Small children in Norwegian asylum-seeker reception centres. In: Childhood 16 (3), S. 395-411.

White, Allen \& Bushin, Naomi (2011): More than methods: learning from research with children seeking asylum in Ireland. In: Population, Space and Place 17 (4), S. 326-337.

White, Allen (2012): ,Every Wednesday I Am Happy': Childhoods in an Irish Asylum Centre. In: Population, Space and Place 18 (3), S. 314-326.

World Vision \& Hoffnungsträger Stiftung (2016): Angekommen in Deutschland. Wenn geflüchtete Kinder erzählen. Friedrichsdorf: World Vision Institut. 


\section{"It's child's play - or is it?" Play, conflicts and relationship networks among children in a reception centre}

Penelope Scott \& Thi Huyen Trang Le

[...] before we turn the corner into the backyard, we hear children laughing and screaming with delight. We soon see why. A sprinkler, oscillating from left to right, is spraying water into the air while fully clothed children are running to avoid its reach or running directly into it. Some are completely wet [...] A woman approaches us holding the hand of a young boy. He has a bruised eye and his cheeks are wet with tears. She explains through our interpreter that some children pushed him down while playing and he hurt his eye. The mother looks visibly distressed as she demonstrates with her hands how the little boy was pushed to the ground (Field visit 5, 22.06.2016).

\section{Introduction}

The laughter, tears and regrettable injury depicted in this excerpt reflect the emotional dynamics and physicality often characterising children's play. The scenario may seem so familiar as not to appear inconsistent with adults' imagination of children playing anywhere. However, the backyard referred to above is not just 'anywhere'. It is located on the premises of a large, converted care home that now serves as a reception centre for refugees in Berlin. The building, which had previously accommodated the frail elderly and infirm, now houses over five hundred men, women and children from countries including Afghanistan, Eritrea, Iraq and Syria. Approximately half of the children (ninety-one), are young children under the age of six. As preschool children their ascribed social location is the physical structure of the reception centre. Their social worlds are constituted primarily by space, family relationships and interactions with other children and adults, many of whom are strangers who do not speak their native language(s). How do these young children experience play, a principal form of their participation in everyday life (Lester \& Russell 2010), and what other experiences with people and places make up the fabric of their daily life within the reception centre? These are the questions which this chapter 
addresses. A focus on the children's lived experiences not only provides insights into how childhood in a reception centre is structured through legal and bureaucratic processes. It also reveals how young refugee children as social actors engage with and influence the conditions defining this childhood. These portrayals are grounded in a dual theorization of refugee children as social actors. First, as children, they are conceptualised as relational, experiencing subjects, with their own perceptions of the generationally ordered social world they inhabit, and with capacities to act intentionally to secure their interests, i.e. to exercise agency. Second, as refugee children they occupy a distinctive marginalised structural location in a highly relational social universe (Bourdieu 1990). The structures and social relations defining their position of socio-economic disadvantage constitute both the context for and the constraints on their perceptions, and capacities to act. Children's practices in daily life, therefore, are produced out of a negotiation with social structures and adult power that seek to reproduce their status as refugees and children.

Children's experiences of play provide the lens through which their daily lives and agentic acts are examined. Play ${ }^{1}$ can be regarded as "an intersubjective space in which fun is central [...]. It consists of relations (of inclusion and exclusion) with other people; with places and objects such as toys, play equipment or the play environment itself; and with the imaginary [...]" (Meire 2007: 2). Play is enshrined as a right for all children in the Convention on the Rights of the Child (UNCRC $\$ 31$ ); its articles collectively offer guarantees of protection, participation and provision. Play's relationship to cognitive development, health, well-being and resilience make it an important mechanism for protecting children; when children play, they engage in a form of self-protection by creating their own well-being (Whitebread et al. 2012; Lester \& Russell 2010). The dominance of play as a form of children's engagement in everyday life makes play consistent with the UNCRC articles on participation. Its importance in children's lives requires the provision of appropriate social and physical environments to support their capacity to play (Lester \& Russell 2010).

\subsection{Locating the 'preschool refugee child'}

Daily life for young children in the centre is uniquely bound up in the production of space. Their everyday life and experiences of play are shaped by three inter-relating aspects of how space is socially produced (Lefebvre 1991). This includes first, representations of space, which refer to the institutional maps

1 For an overview of academic debates on definitions of play see also Eberle, Scott G. (2014). 
conceptualizing appropriate physical places for children in the centre. These maps incorporated the private domain of the one room children shared with their families, and three public places designated as dedicated spaces for children: a playroom, referred to by staff as the "kindergarten", ${ }^{2}$ that was equipped with an assortment of toys and play resources; a corner at one end of the reception area where children could romp; a sand-pit with a small slide and a playhouse located outdoors in the centre's backyard.

Second, spatial practices related to these public spaces set expectations for children's behaviour and usage of these designated sites in the centre. These practices included, for example, opening the kindergarten daily between 10:00 to $12: 30$ for preschool children and in the afternoon from 14:00 to 16:30 for both preschool and primary school children. These spatial practices were intended to establish a sense of order and continuity to children's everyday life by fixing them in time and space within the institution's organisational structure. Other spatial practices had distinctly disciplinary functions and revealed the different actors possessing the power to regulate children's spatial autonomy. The centre administration declared the building's forecourt off-limits to children playing football, unless they were supervised by a childcare worker. This guideline circumscribing children's spaces in the centre was supplemented by rules established by the private security personnel working at the home. Children were forbidden for example, to play in the corridors. They also had to be quiet and refrain from boisterous behaviour in the reception area.

While the centre staff's representations of space conceptualised children's spaces in specific ways and a range of spatial practices were employed in their realisation, children inhabited and used space within the centre in their own unique ways. It is this third, experiential aspect of space - children's spaces of representation - involving their everyday experiences of play, social relations and practices in an adult imposed order which provide the heuristic framework for analysis. The study findings presented and discussed in this chapter show that in the various spatial domains of the reception centre children were actively engaged in seeking out, creating and at times defending spaces for play. This pursuit was mediated by children's individual biographies, and stressful socio-spatial circumstances that injured their rights by undermining the selfprotection function of play.

2 The centre's "kindergarten", however, was not organised according to standards for nursery and day care centres set by the Berlin Senate for Education, Youth and Families (Senatsverwaltung für Bildung, Jugend und Familie). There are currently no compulsory standards regarding the type and quality of childcare facilities provided in accommodation for asylum seekers/refugees. 


\section{Methods and participants}

The study constitutes a form of engaged ethnographic research as it aligns social critique with a commitment to reciprocity in the field (Low \& Merry 2010). The research methods employed make young refugee children's experiences visible with the aim of promoting social justice through critical reflection on their "representation and positionality" (Huschke 2015: 55). The study's interpretivist, qualitative research approach is informed by methodological principles and tools of constructivist grounded theory ${ }^{3}$ (Charmaz 2014) and situational analysis (Clarke 2003). Data collection and analysis were facilitated by a situational map, specifying "the major human, nonhuman, discursive and other elements in the research situation" (Clarke 2003: 554), that served to stimulate analysis of the relations between these elements.

The findings presented in the following section are derived from three sources of data collected during thirty-three visits to the centre over the eightmonth fieldwork period from May 2016 to February 2017. The data include first, observational notes of visits made to the kindergarten, the families' room and areas inside and outside of the centre where children congregated or played. These observational notes also comprise written records of conversations held in the field with a variety of actors including the childcare personnel, social workers, the centre manager, security personnel and parents. Second, semi-structured interviews were conducted with the centre manager, the childcare manager - who was interviewed twice - and a childcare worker. All interviews, with the exception of the first interview with the childcare manger, were recorded. Third, semi-structured interviews were held with the three sets of parents of the five children participating in the project. Interpreting support was provided by a Farsi native speaker, a common language of the participating families, who all came from Afghanistan. These interviews, which were recorded, were thus bounded by "triple subjectivity" (Temple \& Edwards 2002: 6). The observational and interview data were analysed inductively with the assistance of the MaxQda software programme. The data coding entailed the identification of first, open codes, and second, the development of more analytically incisive focused codes, and the creation of more abstract analytical categories (Charmaz 2014).

Of the four different types of accommodation facilities for asylum seekers contacted, the reception centre was the only facility that agreed to participate

3 Constructivist grounded theory "acknowledge(s) subjectivity and the researcher's involvement in the construction and interpretation of data" (Charmaz 2014: 14). 
in the research project. ${ }^{4}$ Recruitment of the families was initiated through contact we established with young children during visits to the kindergarten. We subsequently approached their parents, with the help of an interpreter, to solicit their participation and their children's participation in the project. All the parents we approached gave their consent. The child participants ${ }^{5}$ include sixyear-old Taban and her four-year-old sister Alima, who lived together with a baby brother and their parents Mr. Durrani and Ms. Sanjari. Six-year-old Farah, the last of six children, lived with her mother Ms. Jafari and three older brothers. The sisters six-year-old Saba and four-year-old Razia shared a family room with their seven siblings and parents Mr. \& Ms. Noorzai. The families had been living in the reception centre, which was run by a social care agency, since December 2015. By law, ${ }^{6}$ the families should have been transferred to a collective accommodation centre after six months. However, due to a shortage of housing for refugees, they had to remain indefinitely in the reception centre, which was equipped only for short-term stays.

\section{Findings}

\subsection{Organisational rules and operational realities in the kindergarten}

According to institutional guidelines, the centre's kindergarten had to be staffed during opening hours by one of the childcare workers $(\mathrm{CW})$, who was usually assisted by interns (INT), volunteers registered in a federal volunteer service scheme (FSV), or volunteer youth workers (VYW) participating in a voluntary social year programme. The kindergarten was conceptualised primarily as a space for non-directed play, and its operation was not explicitly guided by educational principles. The manager of the childcare team argued that the introduction of pedagogical approaches was neither wanted nor appropriate, and instead "children should go to a real kindergarten". Of the sixtyfive children eligible to attend a state certified kindergarten, however, only twenty-six had a place. This was unsurprising given the known shortage of places in Berlin (Senatsverwaltung für Bildung, Jugend und Familie 2017).

4 The other facilities contacted included a collective accommodation centre, an emergency accommodation centre and a centre for vulnerable groups e.g. single parents with minors.

5 The names of all participants have been changed by the authors.

6 Asylum Law §47. 
The kindergarten operated according to a series of guidelines and disciplinary rules that established age and behaviour as criteria regulating children's access. Although it was open in the mornings from 10:00 to 12:30 solely for preschoolers, only the three to six-year-olds could attend unaccompanied by a parent. Attendance by the under three-year-olds was conditional on a parent being present. The centre management claimed they had no duty to supervise very young children because the level of supervision they required was greater than its limited personnel resources could provide. The childcare team had a simple but stringent approach to conflict among children in the kindergarten: according to the team manager, if any child hit, spat, kicked or shouted at another they had to leave the room. Exclusion was the penalty for disruptive behaviour.

The logic of the kindergarten's opening hours together with its spatial configuration dictated how children inhabited the play space assigned to them. Two and a half hours daily were dedicated to the exclusive access of children up to age six, and one room catered to young children at various stages of physical growth and emotional development - babies, toddlers and older preschoolers. While children appeared to find their own niches and activities, there were times when the older children, and particularly the boys, showed a greater dominance over certain play resources and use of space in the room:

The room fills up and I count at least eighteen children. It is loud. A group of three 'older' boys monopolises all the bobby cars as well as the trainer bike. They drive/ride energetically around the room [...]. Little Omar is standing close to the chair where his mother sits and watches wide-eyed as the boys move recklessly around the room, weaving their way through whatever spaces open up in their path [...]. Omar looks longingly at the trainer bike one of the older boys dismounted and leaves lying on the floor. He seems hesitant to use it. He accepts my outstretched hand and I lead him to the bike and help him on it (Field visit 4, 16.06.2016).

Overcrowding in the kindergarten at unpredictable times frequently occurred. The congestion this created is significant due to its likely impact on preschoolers' behaviour: overcrowding is associated with social withdrawal, increased aggression and conflict as well as diminished cooperation (Evans 2006). When the room became crowded, particularly during the holidays or inclement weather, it was difficult to maintain an overview of which children were present and whether they met the age criterion for access to the room. As most of the children had brothers and sisters, it was not uncommon for either older or very young siblings to frequent the kindergarten morning session as well. Twoyear-old Abed often went into the kindergarten with his sisters. While there were often parents in the room who had accompanied a child or children under the age of three, there were instances when this did not occur: 
A man pushing a little boy in a stroller enters the room and puts the infant on the floor. I start a conversation with the man, who turns out to be the father, and learn the child's name is Amir and he is one-year old [...]. The father leaves the room and Amir starts crawling around the busy room with a smile on this face. I watch as he crawls quite quickly towards the door and crosses the threshold into the corridor. I was just thinking I should go and get him, because no one else seemed to have noticed, when he does a U-turn and crawls back into the room (Field visit 4, 16.06.2016).

The mixed age groups of children frequenting the kindergarten proved challenging as this increased the risk of accidents. A childcare worker noted that the school-aged children sometimes played so roughly that they would accidentally knock over or hurt the younger ones. When the kindergarten was full, the risk of accidents was particularly present:

While talking to (CW_Male), we both notice a small child has climbed up onto a table and totters as he stomps around on it. (CW_Male) quickly grabs him and places him back on the floor while commenting that it is especially difficult now in the holidays, with the increasing number of children in the room, to keep an eye on all the children. He added that [...] those under three, shouldn't be left in the room, a parent ought to be there and "they knew the rule". The little boy he had just rescued from the top of the table was two years old (Field visit 10, 28.07.2016).

Just what parents understood - or remembered - about how the kindergarten operated is open to question. The childcare team did not have a policy of regular scheduled meetings with parents. According to the childcare manager, information about the kindergarten was communicated orally to parents by a social worker during their official induction into the centre on the day of their arrival. It was evident, however, that some misunderstanding about the rules of the kindergarten did exist. Four-year-old Alima stopped attending the kindergarten when her older sister started school. The little girls' mother, who gave birth to a baby boy soon afterwards, withdrew Alima from the kindergarten due to apparent confusion about the rule that parents must accompany younger children. She stated in an interview a few months later that because she had to take care of the baby, she found it impossible to remain with Alima in the kindergarten. Instead, the child stayed in the family room with her mother and baby brother, while her father went to German class and her older sister went to school. Alima's days consisted mainly of watching television, watching over the baby while her mother cooked in the communal kitchen, and waiting for her older sister - and main playmate - to come home from school. By the middle of winter, the walls inside the small family room had been transformed into a canvas: Alima had scribbled and drawn all over them as she adapted to her new routine and found creative endeavours to occupy herself. 


\subsection{Playtime in the kindergarten: dealing with linguistic diversity}

The kindergarten had an important social function in the centre as a recreational meeting place for children, where they interacted with their peers as well as the childcare workers and their assistants. The children were plunged, however, into a multi-lingual environment in which the adults communicated with them in German, and their playmates may or may not share their native language(s). This held true also for children from the same country such as Afghanistan, Syria and Iraq, where nationality was not tied to one single language. Razia and Saba's family language was Uzbeki, one of the several minority languages spoken in multi-ethnic, multi-lingual Afghanistan. Their siblings' Afghani playmates were mainly Dari speaking, however, and as one of them commented in Razia's presence "she doesn't understand me when I speak to her" (Field visit 10, 28.07.2016).

The childcare workers and their assistants - monolingual German nativespeakers - did not raise the existence of a language barrier as an issue affecting their work or mediating their relationship with the children. They all claimed that the children "learn German quickly". There were instances, however, when the children were spoken to at a volume that appeared disproportionate to the size of the room or the prevailing noise level:

[...] it was already 12:20 and the kindergarten was due to close at 12:30. Suddenly, I hear (VYW_Female) and (INT_Female) say very loudly "tidy up!', which they repeat several times. Some of the kids just run out of the room, others start to pack away the toys while others just continue playing (Field visit 4, 16.6.2016).

One of the interns often shouted at the children or spoke to them in a severe, bossy tone when giving instructions or reprimands. While this behaviour may be indicative of other adultist ${ }^{7}$ practices within the centre, increasing speech volume and modulating tone of voice are strategies sometimes used in communication with non-native speakers in efforts to facilitate the process (Fontes 2008). The opposite effect may be the outcome, however, as tone and volume can be interpreted as threatening, particularly when the verbal content of the communication event cannot be understood. Studies prove that early childhood is an optimal stage for children to learn another language, but children's language abilities - in their own native language(s) as well as in the new language - do vary considerably (Essen \& Taheri 2016). There could be no certainty, therefore, what the children in the kindergarten understood when they

Adultism is the abuse of the power adults have over children and refers to behaviours, attitudes and practices based on assumptions that children are inferior to adults. See Flasher, Jack (1978). 
were spoken to in German. The younger participants in the project, four-yearold Alima and Razia, appeared to understand less German than their six-yearold counterparts, particularly in the first six months of the project. They often stared blankly at us when we spoke to them in German.

\subsection{Sharing and competing for resources in the kindergarten}

There was no specific financing dedicated to covering the material resources needed in the kindergarten. Everything in the room - the furniture, toys and play equipment - had all been donated and were mostly second hand. It was the task of the childcare team to select from these donated items what was still reusable and age appropriate for the children. Funding for small purchases could be procured from the centre's limited household budget. However, this budget had to cover all recurrent expenditure in the centre; every request for financing had to be assessed, as the manager claimed, to determine "what was absolutely necessary". This automatically excluded, for example, the possibility of buying several of the same toy or game that proved popular among the children. Even creative material such as colouring books, drawing paper and crayons were first sought from possible donors. The lack of dedicated financing for the kindergarten and dependency on donations consequently created the conditions in which children had to share or compete for their favourite play things.

At times children played peaceably together, sharing a particular toy, game or craft material:

I wander over to the group of children nearest to (VYW_Female). The five little girls are sitting or squatting, huddled together, on top of a small table and are occupied with a colouring book. One of the little girls is colouring a tree while the others watch intently. I point to the tree and say to her "that is a tree" and then unexpectedly, all the little girls repeat the word 'tree' in a chorus! (VYW_Female) overhears and joins in. She starts identifying different objects pictured in the colouring book. The little girls all repeat the words (Field visit 4, 16.6.2016).

The sole colouring book and only visible crayon were insufficient for all the little girls to participate in an activity that not only offered fun but provided educational benefits, such as the strengthening of fine motor skills. The steadfast concentration of the little girls, however, as they watched their playmate colouring the tree, demonstrated a keen interest in observing ways of mastering the activity and discovering how the empty outlines on the page could be transformed by the application of colour. Their unsolicited repetition of the German 
word for tree was further indicative of their receptiveness to learning and their openness to participating in the process.

Conflicts - interpersonal exchanges characterised by oppositional positions in which verbal or physical aggression may or may not be present (Laursen \& Collins 1994) - did occur among children resulting from competition for the use of different resources. Although this was consistent with behaviours expected in their age group from a developmental perspective (Parker et al. 2006), research shows an increase in preschool children's aggression in conditions characterised by overcrowding and a low quantity of play resources (Evans 2006). However, conflict was not the inevitable outcome of competing claims over play resources:

Taban followed by Alima and another little girl come marching up to me. Taban takes me by the hand and pulls me towards a corner of the room where there was a play stove and washing up sink. Two little boys - probably about their age - are sitting in front of the stove playing with the plastic saucepans on top of it. Taban looks at me, points to the stove and sink - while talking in Dariand then points a finger to herself and the other two little girls present. I have the distinct impression she wants me to send the boys away, so they can play there. (...). The little boys look up at me expectantly with their eyes wide open. I say the boys can play for another "two minutes", and I demonstratively hold up two fingers, after the two minutes they should "share" and allow the girls to play. I move away and stand nearby but the girls remain resolutely where they are. Within what seemed like a few seconds, Taban looks over at me and says, "two minutes", as if she felt the time had already elapsed. I say to her "another minute" and the little boys keep on playing, appearing oblivious to what else is happening around them. I wait a little longer and then say to the boys "it's the girl's turn now". Without protest, they get up and leave (Field visit $8,11.7 .2016)$.

Taban found a creative solution to the children's lack of control over access to limited play resources. She asked an adult to intervene on their behalf. But rather than approach the childcare workers, who enacted discipline in the kindergarten, she solicited a researcher, probably with the expectation that the personal relationship they shared was more likely to guarantee the fulfillment of their demands. This was a form of proxy agency, intentional action by an individual(s) that gets others perceived as having influence and power to achieve outcomes they desire (Bandura 2001). This act, which emerged out of the relational ties between Taban and the researcher, underlines the importance of relationships between actors within children's networks as an enabler of their agency (Betz \& Eßer 2016). Significantly, in accomplishing this agentic act Taban also used a rhetorical strategy: repeating "two minutes" - the temporal marker indicating when the girls' could access the stove - as a reminder of their claim to it. 


\subsection{Conflict and physical aggression during play time}

Physical aggression, often in the form of hitting, did accompany conflicts developing between the children while they played. Parents stated during the interviews that their children had complained that other children had hit them, although they usually could not identify the perpetrators by name. In two separate incidents, Taban and Alima's parents were able to establish the identities of the boys involved. They said they attempted to broach the issue with the boys' parents. However, the encounters did not end favourably and resulted in tensions developing between them and the respective parents.

The childcare workers also reported that hitting was a regular occurrence. They attributed children's behaviour to their "culture" and the "trauma" of having to flee war and persecution in their homelands. The childcare manager articulated this position as follows:

"It wouldn't leave me speechless if children hit adults. The children have been through a lot and in their culture, it is normal to hit. This mentality won't change just because they are now in Germany" (Interview, 3.03.2017).

The staff noted that conflicts between the Arabic speaking and Afghani children were a problem. They claimed the privileging of Syrian refugees in the asylum application process and their quicker access to integration courses had led to animosity between the various ethnic groups. As a consequence, the children were viewed as acting out the hostility existing between the adults. The 2015 asylum reforms did lead to the prioritizing of asylum applications from nationals of Syria, Eritrea, the Balkan countries and Iraqi religious minorities (Kalkmann 2015). The inclusion of nationality-based distinctions into legislation in effect created a situation of unequal competition for asylum among the various ethnic groups applying. ${ }^{8}$ These distinctions were manifest in the provision of bureaucratic services in the centre. A childcare worker commented, for example, that there was more material available in Arabic and greater translation support for Arabic speakers. By contrast, there was less material and support available in Farsi for the Afghanis. It was, the childcare worker said, as if this was due to the forgone conclusion "that they would be all deported anyway".

Parents claimed in interviews that although their children had specific playmates that they liked to spend time with, they played with other children regardless of their ethnic identity. However, as one parent commented, "sometimes the children have fights". Farah's mother maintained that when conflicts occurred there were usually several reasons why, and "ethnic group did not

8 It also "contradicts the 1951 Refugee Convention and human rights obligations, which require equal treatment in the provision of rights and guarantees to all persons seeking international protection." See Mouzourakis, Minos (2016). 
play a role". While we did not observe any inter-ethnic conflicts among children, we did observe instances of conflict involving physical aggression between children of the same nationality. One such episode occurred during the morning session of the kindergarten when the children were playing in the sandpit under the supervision of two interns:

(INT_Male) and (INT_Female) are talking to each other near the entrance to the building about thirty meters away from the sandpit. I remember again what the childcare manager told me on the first day - that I would never be alone with the children! [...] Taban is playing with a plastic watering can [...]. Suddenly, I hear a child shouting and I see Farah grab the watering can from Taban and hit her on the forehead. Taban falls backward and hits her head on a dolly stroller. She starts howling and holds her forehead. [...] (INT_Male), who heard the howling, comes over and I tell him what happened [...]. (INT_Female) comes and wants to know what happened [...]. She calls out to Farah three times by name in a stern voice, but the child does not look up at her. Instead she sits there motionless, with her head hanging down. [...]. (INT_Female) asks how long ago the incident took place and (INT_Male) says about ten minutes - which would have been my estimate too. I add that I had asked Farah to excuse herself, which she did, and the children have been playing peacefully since then. (INT_Female) says that hitting, spitting and kicking are not tolerated and the children must go back to their room when that happens. (INT_Male) looks at her and shrugs his shoulders as if he thinks the matter is settled so there is no need to intervene. (INT_Female) responds by saying "the rules apply outside in the yard as well as inside". She knits her brow in annoyance while saying that the child is "rude" and that she hadn't even looked up at her even though she had called her three times. (INT_Female) picks the child up, removes her from the sandpit and tells her to go to her room, while pointing in the direction of the building (Field visit 6, 1.7.2016).

This episode reveals the childcare staff's differing attitudes towards the kindergarten rules and the physicality involved in enacting discipline among the children. It is also illustrative of how children's unique biographies can mediate their experience of play and interactions with their peers. Farah's behaviour had proved disruptive before in the kindergarten. At times, she was at the centre of disputes among her playmates that resulted in her being reprimanded by the staff. She also exhibited what a staff member termed "negative ways of getting attention" - such as standing on the tables, which was against the rules, or opening cupboards that were off limits to the children. The childcare workers attributed Farah's general behaviour and physical aggression to her "family history" and her mother's incapacity to fulfil her parental role due to her own poor mental health. They claimed Farah had told them her mother was "always sad and cries a lot". The source of the mother's distress were events occurring during the family's difficult journey to Europe. While crossing over the Iranian border into Turkey with a large group of people, the single mother of six got 
separated from two of her children. They were among several persons apprehended by the border police. The children - both teenagers - eventually managed to find shelter with a relative living in Iran.

Farah's mother, Ms. Jafari, spoke during the interview about the severe difficulties of their journey to Europe, and two other prior attempts the family had made to leave Afghanistan. She confessed to feelings of guilt over being separated from her other two children, and to her worries that they would attempt the perilous journey to Europe on their own with the help of smugglers. She complained she often had headaches due to the stress of her situation. A doctor had prescribed medication, which made her drowsy, and had told her she was "in danger of developing a depression". Ms. Jafari acknowledged her health status sometimes affected her ability to fulfil her domestic responsibilities, and she was aware it also had an impact on her relationship with Farah. She claimed she often "did not feel like playing" with Farah, and added the child had responded defiantly when she asked her not to go to the backyard by saying:

"I can't sit here and cry with you, I have to play. You can't play with me so that's why I'm going to go downstairs and play with others" (Interview, 5.10.16).

Farah's decision to seek out the companionship of her peers and to play is an agentic act demonstrating the self-protection function of play in fostering children's well-being (Lester \& Russell 2010). Ms. Jafari's admissions lend credence to the childcare workers' opinion that Farah's behaviour was related to her mother's fragile mental health status. A link is plausible given empirical evidence on the effects of parental, especially maternal mental health on children's behavioural and mental health problems (Valloton et al. 2016; Fitzsimons et al. 2017), and the use of physical aggression by children to externalise problems (Card et al. 2008). However, it is debatable whether the child's behaviour can be explained exclusively in terms of her mother's inability to fulfil her emotional needs, as a childcare worker stated in an interview. Ms. Jafari noted that Farah was afraid to go on her own to the toilet, which was at the end of a corridor, and she still talked about the family's first attempt to cross the border to Turkey in a large haulage vehicle transporting foul smelling sheepskins. The difficulties of her present living circumstances in the centre and her past experiences on the long way to Europe were also factors affecting the well-being of the little girl. 


\subsection{Temporal disruptions, temporal uncertainties and relationship ruptures in the kindergarten}

The opening hours of the kindergarten established order to the general functioning of the centre reflecting the way time is bound up with routines, schedules and work practices of institutions (Edensor 2006). The opening hours consequently created expectations among the children, and the parents for whom the time-space of the kindergarten had its own significance. For the parents, the time children spent in the kindergarten could mean a brief respite enabling them to attend to other domestic chores or bureaucratic appointments. It was also valued as providing a safe play environment for the children that preempted a series of problems, as Mr. Noorzai said:

"Thank God there is a kindergarten, otherwise I would have a lot of problems here. If there wasn't a kindergarten, then the children would all go outside, they would get into fights with the other children or their clothes would get dirty, or they would get sick or perhaps they would go out onto the street, that is a danger" (Interview, 15.02.2017).

For the children, the kindergarten offered time limited access to play material and equipment. Through interaction and play activities in the space of the kindergarten, they constructed relationships not only with their peers but also with the childcare staff. The affectionate relationship some children developed with staff members was visible in the forms of physical contact the children initiated and the emotions they displayed. Children would sometimes greet the staff with a hand shake and/or an enthusiastic "Good morning" while smiling at them warmly or embracing them affectionately around their legs. There were instances when a child would crawl onto the lap of a worker and allow themselves to be embraced, suggesting the existence of an emotional attachment.

Given the significance of the time-space of the kindergarten, disruptions to its scheduled opening times could alter the temporal patterns and expectations of both the children and their parents. Although its official opening time was 10:00am, at the commencement of the fieldwork we noted the kindergarten usually opened later. The childcare manager explained this as an adjustment to the changed temporal rhythms of the residents during Ramadan. She claimed that the families were going to bed later, so the children would come to the kindergarten later. However, once Ramadan was over, we observed over the fieldwork period that the kindergarten still followed this pattern of opening late, and it was sometimes closed during its scheduled opening times. On one such occasion, a group of children and a parent were standing outside the kindergarten at approximately 14:15 waiting for it to open. An hour later, the kindergarten was still closed. 
The variations in the opening times can in part be explained by staffing fluctuations and shortages - itself an indicator of how temporality shaped the working conditions of staff and ultimately children's experience of the kindergarten. The childcare workers were issued with six-month contracts and had no certainty that their contracts would be prolonged. A few months into the fieldwork, one of the childcare workers - the only man in the team - left due to this insecurity. For the children, his departure meant the sudden end of a relationship with an adult they interacted with daily in the kindergarten. As the child care manager noted in an interview, "he was there one day and was gone the next". She commented that Farah found it very difficult to come to terms with him leaving. For the remainder of the fieldwork, the vacant position remained unfilled leaving the other two staff members to manage the kindergarten as well as the administrative and care related responsibilities of one hundred and eighty-five children. The childcare manager stated that they were both "overwhelmed" with the workload and extra responsibility. As a result, when there were "too many children" in the kindergarten they sometimes had to shut the door to prevent others entering. This was the only way, she said, that they could maintain "an overview of who was in the room." Aside from that, she added, doing so also served as a "self-protection" mechanism. The staff's reactions to these stressful working conditions therefore, had consequences for children's access to the kindergarten.

\subsection{Children's surreptitious use of space}

Outside the adult ordered space of the kindergarten, children had their ways of appropriating space for play and social interaction. At times, their spatial trajectories contested the dominant social production of space in the centre. Especially when the kindergarten was closed, and inclement weather prevented outdoor play, the open space in the reception area was a favoured site for groups of children to hang out together, and the long corridors were transformed into a playground. These forms of "playful spatial production" (Lester \& Russell 2010: 30) gave a meaning to these places in the centre that contravened security's prescribed representation of space. Security framed these sites as "no play areas" based on a logic of governance and safety: i.e. creating a quiet, enabling working environment for security staff in reception as well as safe, noise-free walkways in the corridors outside residents' rooms. This was at odds with the children's logic that valued space differently and regarded the use of available spaces for play as a tactical opportunity for participating in daily life in ways that were appealing to them. These conflicting rationalities in terms of the production of space were known to the centre management staff. In the interviews, they expressed an awareness that some security personnel 
did not have child-friendly attitudes; they also claimed to feel challenged at times by complaints they received from security about the children violating the spatial rules. The staff, however, expressed sympathy for the children's need to play; as the childcare manager said, "where else are they supposed to play when we go home at 16:30?" This position reveals unresolved tensions between the management and security over the production of space in the centre, and signals their differing stance on children's place within it.

\subsection{Play and conflict in clandestine spaces}

The attraction of the long corridors to children was not difficult to understand. It was an easily accessible space, outside the one room they lived in, which held the potential of chance meetings with other children and opportunities to play little games away from the gaze of adults. However, the potential to play also carried the possibility of conflict if unwanted intrusion by other children occurred. The following incident took place as we were colouring with Taban and Alima in the corridor outside the family room, where their exhausted mother was tending to her eight-week-old baby:

[...] the same little boy who had watched us knock on the family's door is now hanging around in the corridor observing us. He moves closer to us and soon is standing at the edge of the sheet we are all sitting on. I wonder if I should ask the little boy if he wants to colour too and then I notice that both Taban and Alima start to get restless. They keep looking up at him. Alima seems particularly distracted by his presence. The little boy moves even closer to us and I hear her say in a distressed tone of voice "no, not good". I ask "why?" and Taban answers "he always hits my sister". Alima gets up and pushes the little boy away. He retaliates by hitting her with both his hands in a frenzied manner. In a split second Taban jumps up and hits the little boy, who starts hitting her back. I get up immediately and put my arms around the little boy to prevent him from hitting and to shield him from Taban. Suddenly, I hear a female voice saying something loudly and sternly in what sounds like Dari. I look up to see an adult woman walking towards us with a serious facial expression. The only word I can understand is Taban's name. Taban sits down again and resumes colouring. The woman takes the little boy by the hand and leads him into a nearby room (Field visit 22, 22.12.2016).

Little is known about the cause of the conflict between the little boy and Alima. Her parents said in the interview that Alima claimed the little boy, who lived in a neighbouring room, had hit her on repeated occasions. During one incident, his father intervened and shouted at Alima. Afterwards, Alima became fearful of the little boy's father too. Alima's palpable fear as the little boy 
approached was indicative of the stress children can experience when confronted by others perceived as a threat. Yet the incident also reveals distinct forms of agency exhibited by each of the child actors in the situation. Pushing the little boy away was a pre-emptive act of self-protection by Alima while her older sister's intervention, in the escalating conflict, sought to defend her sister as the little boy inflicted blows. The sisters' reactions exemplified seeming moral contradictions in the children's behaviour: physical aggression, which can cause harm, was used as a form of self-protection and altruistic defence. It demonstrates, however, that moral agency is operative in children's handling of conflict and that preschool age children have their own ways of dealing with conflict (Danby \& Baker 1998). The reaction of the woman who approached us was to reprimand Taban, in what could appear as a one-sided response to a conflict involving three children. For the little boy, drawing ever closer to us while we coloured was most likely motivated by curiosity and a desire to participate in the activity. Not long after the incident, a man came out of the room and asked if he could have a colouring picture to give the little boy.

In general, a genuine desire for recreational activities and interaction with others, whether playfully or otherwise, was evident among the children we encountered. We were often approached by children, particularly when the kindergarten was closed, who would engage us in light-hearted conversation or ask us expectantly "are you going to have a kindergarten?" Sometimes children followed us to the rooms of the children we visited and would 'hang around' at the door or in the corridor in the hope that they would also be allowed inside to play. If we accompanied any of the participating children outside, other children would 'hang on' and join us as we made our way to the backyard. Children's active pursuit of interaction and recreation was in itself an agentic act; by 'hanging around' and 'hanging on' they demonstrated an eagerness to participate in social life, and they knew how to position themselves strategically to accomplish this.

\subsection{A private domain without privacy: snapshots of life and play in the family room}

As the fieldwork progressed, we noticed that the children participating in the project preferred to spend time with us in their family room, even if this meant leaving the kindergarten or other playful activities they were engaged in outdoors or in the corridor when we arrived. The children would cling to us as we walked towards their family room. If we paused en route to speak with other children, they tugged at our hands and steered us in the direction of their room. This conveyed the impression that they viewed the other children as 
competitors for the individual attention and play activities we offered (see chapter by Scott).

The room was in one regard, a space where the nurturing of relationships and the interactive processes comprising family life took place. It also represented, however, a time-space where the differing temporal rhythms of individual family members, especially babies, dictated how space in the one room was experienced. We often observed exhausted parents trying to nap during the day while children were running around the room chasing each other; or a mother making bread in one corner of a room while a father learned German in another; and we witnessed older, school-aged siblings attempting to do homework while younger ones watched television or jumped around with a skipping rope. Parents stated in interviews that they found living in one room very difficult and certain aspects of their living circumstances affected the children too. Taban's father said the little girl noticed the big differences in their living conditions compared to their home in Afghanistan, and having to use a communal toilet was a particularly unpleasant experience for her:

"She says, where is our carpet and we have so little here. She misses our house and asks, why don't we go back, the kitchen is so far away, and the toilets are not clean, and everybody uses them. She is very sensitive about the toilet and when she goes, she holds her nose and if it is not clean, she comes to me and says 'Papa, please clean it so I can use it'" (Interview, 8.02.2017).

The lack of privacy in the family room had consequences for the children also. Close contact with one another was inevitable while playing in the room, and there was little available space for children to retreat to if squabbles occurred or when siblings tried to intrude upon activities they were engaged in. This was an issue in the family room shared by 4-year-old Razia and her eight other siblings and parents. We often observed her younger brother Abed, an energetic and mischievous 2-year-old, shadow his sister as she coloured, played with a mosaic set or other games that we had brought. Within the confines of the room, it was impossible for Razia to evade Abed's intrusions into her individually created play spaces. They would sometimes play peacefully together but she would often try to prevent his interruptions by turning her back to him or moving a few centimetres away. When these pre-emptive tactics did not work, and she became overwhelmed by his 'pester power', she would resort to physical aggression and fights would ensue. At times Abed's interruptions brought his sister's play to an abrupt end or disrupted it completely by ruining the creative output of her play:

Razia selected a picture with Hello Kitty and starts painting [...]. Suddenly, Mrs. Noorzai shouts at Lalah and Abed, who are sitting on a bed playing with the mosaic set. They both get off the bed [...] and Abed wanders back over (to us on the carpet). He begins swirling his fingers around in the little pot of water in which the children cleaned their paint brushes [...]. He rushes off with the 
pot [...] and then comes back into the room holding the pot that now contains fresh water. He trots over to us and with a grin on his face, pours the pot of water all over the newspaper on which Razia is painting. She stops painting and watches as her Hello Kitty picture dissolves into a watery blur of colours (Field visit 17, 30.09.2016).

\subsection{Environmental and personal safety risks during non- directed play}

The willingness of parents to allow us to spend time with the children in the crowded space of the family room surprised us. During the interviews, it was apparent that some parents had reservations about their children playing outside, which may be a reason why they so generously accommodated us in the family room. Both Ms. Jafari and Mr. Durrani expressed concerns about the safety of their daughters playing unsupervised in the centre. Their concerns were rooted in a general suspicion of the countless, unknown people living there, the negative influence of older children or the likelihood of conflicts developing among the children. This resulted in parents either trying to restrict children playing outdoors or limiting the time they spent away from their supervision:

"When Farah goes outside and plays with others, I don't know who they are and maybe she learns something bad [...] there are so many different people living here, I don't know all of them. It is difficult. When the older boys and girls are also outside playing, I'm always afraid that they will fight, or something will happen [...]. I have said [to Farah], please don't go to the backyard, I'm scared that the people there will fight, or something will happen [...]. The older girls often say to Farah, 'come let us travel with the tram for a few stops and see what there is' [...] nowhere is completely safe. I'm always afraid when Farah goes outside" (Interview with Ms. Jafari, 5.10.2016).

"We're scared because they are just little girls [...]. Usually, we don't let them just be on their own anywhere [...]. We always watch over the children. I let them go outside [...] but I'm careful, I will go and collect them quickly. I don't know the people or the area, so I want to watch over the children" (Interview with Mr. Durrani, 8.02.2017).

The location of the centre also posed certain environmental risks to children's safety as they played. It was situated on a large complex, which also accommodated offices and other institutions. The front of the centre was very close to a main road; only a narrow strip of grass and a low hedge separated the building from a busy street. On several occasions we saw very young children wandering along this grassy area looking over the hedge onto the traffic 
passing by. Sometimes children on bicycles would ride in front of the building and directly into a parking area reserved for office employees. While walking with the childcare workers in the complex one day, we witnessed an agitated employee approach them to complain that a child had cycled in front of his car as he was driving, and an accident had narrowly been avoided (Filed visit 4, 16.6.2016). At the rear of the building, a service road separated the backyard where the children played from another institution. During a morning session of the kindergarten, as the children played in the backyard under the supervision of an intern, Taban drew my attention to two little boys in bobby cars pedalling along the service road (Field visit 6, 1.7.2016). On another occasion, Razia suddenly ran out of the sand pit and onto the road in front of a car that fortunately, was driving slowly (Field visit 16, 15.09.2016). In the backyard, we watched small children under apple trees collecting fallen rotting apples, and we saw Razia nibbling a decaying apple, while she clutched two others. A school aged playmate of her older sibling who was present commented with a grimace, "Oh, the apples are bad, I wouldn't eat them" (Field visit 10, 28.07.2016).

\subsection{Scarcity and surplus of play resources: the contradictory outcome of competition in the field}

A common feature of the family rooms we visited was the noticeable absence of toys, play objects or play equipment. In two of the rooms, a small collection of stuffed toys that the children had received in the first centre accommodating them lined the tops of cupboards. In the interviews, Taban and Alima's parents said the girls had two dolls they hardly played with, and Mr. \& Ms. Noorzai stated the children had a few footballs. Whenever we visited the family rooms, we usually found the children watching television. There may have been several reasons for the absence of play material and equipment: pragmatic concerns about finding the money to buy them and/or the space to store them in the room; rational considerations about the practicality of investing in such purchases due to the uncertainties of their status; and parents' own attitudes towards play supported by toys and play equipment.

In contrast to the apparent scarcity of play resources in the family rooms, the centre was in possession of a storage room containing donated toys that the childcare staff claimed they did not need and planned to pass on to another accommodation centre. When we enquired in the interviews why they preferred to do so rather than offer the play material to the residents, both the childcare manager and centre manager described this as not being a feasible option due to the extreme difficulties of distributing resources fairly among the residents. The childcare manager cited two examples to support her claim. 
First, they once set up a table with surplus toys and games from which the residents were told they could select one item per family. However, the residents apparently "all wanted the same thing", some parents took several toys and some persons took toys even though they did not have children. Second, at the Christmas party, when prizes were distributed to the winners of games, some children were not happy with what they got compared to what others received, and some children could not understand why they did not get anything. The result was bickering, crying and tussles over the prizes.

The centre manager added that they "had to be very careful about whom they gave what because the competition among them (the residents) is extreme". She explained this phenomenon among the children as an extension of sibling competition occurring in "normal families" but also saw it as an outworking of the animosity existing between the Syrians and Afghans due their differential treatment in the asylum system:

"It's naturally difficult between these two main groups, the Syrians and Afghans $[\ldots]$ and unfortunately, the children have picked this up so we repeatedly notice that the children always use this as a way to argue, they [the Syrian children] always say 'the Afghanis hit us' and then they [the Afghani children] say exactly the same about the others, but the parents are unfortunately, also the same $[\ldots]$. And then one person doesn't understand why the other is granted asylum but they aren't, and politics plays a role here, unfortunately. Then there is a group that they both have the same opinion on - the Eritreans, they both think they have no reason to be here [...]. Politically, it's of course the Syrians who, let's say, relatively easily get a residence status while for the Afghanis, it's as if they shouldn't be here and should go back" (Interview, 17.03.2017).

\section{Discussion}

This chapter has engaged with the issue of children's experience of play in a reception centre as a window to their participation in daily life. These experiences are inherently spatial and are co-produced through bureaucratic, adult centred views on how spaces for children in the centre are conceived (representations of space), spatial practices that aim to fix children within the timespace of its organisational structure, as well as the ways in which children inhabit and use space (spaces of representation). Given the political and socially constructed nature of space (Lefebvre 1991), children's everyday participation within the centre reflects how state asylum laws infiltrate daily life by structuring opportunities to play and affecting social interaction among children and between children and adults. Significantly, the state's role is bound up with human rights obligation to ensure the provision of a social and physical 
environment supporting children's ability to play and to participate in social life. The findings of this study highlight, however, the ways that children's provision rights are undermined, thus supporting other studies demonstrating the unsuitability of mass accommodation centres for children (Lewek $\&$ Naber 2017; Seeberg et al. 2009). Specifically, these provision rights were infringed through a constellation of factors that included first, insufficient physical space in the kindergarten to accommodate the age specific needs of children under six; a consequence was frequent overcrowding of the room, which increased the risk of accidents. Second, staff shortages resulted in reduced opening hours of the kindergarten and increased pressure on the childcare staff who were at times overwhelmed by the demands of the job. Third, the centre's proximity to traffic posed environmental safety risks. Importantly, there were no communication structures allowing the voices of children to be heard, which violated their participation rights guaranteed by the UNCRC.

As social actors, children engaged in a range of practices showing how they adapted to the limitations of their social and physical environment. These included colonising open spaces in the reception area; taking advantage of cracks in an adult imposed order by playing surreptitiously in corridors; or 'hanging around' and 'hanging on' in the pursuit of recreation and interaction. This demonstrates children's capabilities to be "active in the construction and determination of their own social lives" (Prout \& James 1997: 8). However, given their subordinate position in relationships to adults (Mayall 2000), children's play experiences were also affected by their parents' attitudes. Some parents were fearful and saw threats to their children's safety from both adults and other children living in the centre. Consequently, they closely monitored children's activities and restricted their spatial independence.

The study findings further show that while pursuing playful activities and interactions with others, children are exposed to a range of stressors i.e. challenges, demands, and conditions of threat that tax or deplete capacities to cope (Wheaton et al. 2013). Being immersed in the multi-lingual environment of the kindergarten, where children are exclusively addressed by childcare staff in German, deserves recognition as a new experience confronting children that is potentially taxing. The childcare workers' dismissive attitude to the issue, evident in their claims the children "learn German quickly," does not acknowledge the challenges inherent in environments characterised by linguistic diversity (Gogolin, 2002). It reflects a "monolingual habitus" - a perceived "common feeling for language" (Gogolin 1997: 40) situating common-sense beliefs about language acquisition in the childcare workers' communication practices. The influence of the monolingual habitus is implicated in adultist practices engaged in by some childcare staff, such as speaking loudly, using a harsh tone of voice and shouting at the children in order to make themselves understood. Such practices may be threatening to children. They further constitute forms of symbolic violence (Bourdieu, 2001) as the children, the 
subordinate actors in a hierarchical relationship, submit to their domination because of the childcare workers' legitimated authority in the kindergarten.

As play is a situated activity, in which children as social actors create their own well-being by engaging with their environments, unwanted interruptions to play rupture this process. Yet the likelihood of such interruptions was a real possibility in the cramped private space of the family room and in public spaces where there was limited retreat from the scores of other children living in the centre. Children had to play under conditions in which the threat of interruptions by others, which could lead to conflicts and reprimands from adults, as well as fears of other children and adults, were ever present. When conditions are threatening, they are also stressful (Pearlin 1989); as such, children were confronted in daily life with stressful circumstances as they played that had the potential to undermine play's self-protecting function.

These findings on the stressors and adultist practices characterising preschool children's experience of life in the reception centre draw attention to the impact of their social and physical environment. These circumstances have a significant role to play in the children's well-being, particularly through modalities that either support or undermine their ability to play. In such a resource poor setting, therefore, the surplus play material possessed by the centre contrasts sharply with the shortage of toys and play equipment experienced by the children. The staff's justification for not distributing the excess resources due to fears of exacerbating perceived inter-ethnic tensions between Syrians and Afghanis is itself significant. This position shows the incalculable ways that the reforms introducing nationality-based distinctions into asylum legislation are implicated in configuring micro-level bureaucratic practices. It also reveals the discretionary power of the staff to make unilateral decisions about public donations intended for the residents. The power effects (Keller 2013) of the discursive construction of inter-ethnic strife among adults and children therefore, ultimately included depriving the children of toys and play material.

The practices associated with discursive constructions of young refugee children by centre staff members is an area requiring further enquiry. The childcare staff's racialised explanations of conflicts among children as attributable to their culture, and as an outcome of the traumas of flight, also warrant critical scrutiny. By invoking culture and trauma, staff constructed a homogenizing picture of the refugee child as pathologised as well as culture bound, and so prone to physical aggression. Children's experiences of war and flight deserve serious attention as factors exerting an influence on their emotional and mental well-being. The study's research findings show that some children are still preoccupied with the extreme hardships of their journey to Europe. However, the staff's essentialist and racialising discourse on conflict fixes children in their past and fails to acknowledge the likely impact of the present on their well-being and behaviour. In this regard, the findings of this study highlight the stressful circumstances young children experience in everyday life, 
and the ways in which their provision rights to play and participation are restricted. Rather than using physical aggression gratuitously, our observational findings show that in situations of competition over play resources, children could employ other practices, such as proxy agency accomplished through relational ties, and rhetorical tactics to resolve the situation. Children's physical conflicts also demonstrated their capacities to act as moral agents, with aggression having the functional role of protecting self and defending others. In certain instances, therefore, conflict constituted an agentic strategy by young children to deal with perceived threats in their environment. Consequently, their behaviour reveals how they interpreted their social world and their position within it. These acts sought ultimately to alter the conditions of their childhood, and utilised forms of contestation that challenged normative, adult assumptions about appropriate behaviours in otherwise inappropriate places for children.

\section{Conclusion}

This study's findings illustrate the social conditions of childhood for preschool children in the space of a refugee reception centre. The research also demonstrates the individuality of young children, thus challenging static, homogenizing constructions of the 'refugee child'. The children participating in the study were all unique in terms of their biographies. Their uniqueness was also evident in the type(s) of play they enjoyed: some liked rough-and-tumble play while others preferred play with creative materials, or games. What characterised all the child participants, and the other children we encountered, was an enthusiasm for participating in daily life by playing, learning and interacting with others. Nurturing these characteristics is the necessary foundation for their well-being but it requires a more wholesome and stimulating environment. Institutional forms of refugee accommodation such as reception centres do not provide the right setting for the fostering of children's development and the fulfilment of their rights. Private housing for families with children should instead be the accommodation standard. Furthermore, Article 21 of the EU Reception Directive requiring the recognition of single parents with minor children as a vulnerable group with special needs should be fully implemented. When the accommodation of children in collective accommodation facilities proves unavoidable, strict legal limits to the length of their stay should be established. Additionally, obligatory standards should be set for the type and quality of childcare available, training and support offered to childcare staff, and a complaints procedure should be put in place according to $\S 45$ SGBVIII. ${ }^{9}$

Social Code Book VIII - Child and Youth Welfare. 
The implementation of such measures is crucial in view of the study's findings demonstrating the importance of the kindergarten as a space where young children could create their well-being through play, build relationships with others and so learn important social and physical skills, as well as develop their cognitive abilities. Yet the kindergarten was inadequately resourced, particularly regarding personnel. Its low priority in the centre was evidenced in the lack of dedicated funding for its operational running costs. Given the shortage of regular kindergarten places in Berlin, the case for improving the standards and resourcing of childcare facilities for young children in accommodation centres is even more compelling.

\section{Bibliography}

Bandura, Albert (2001): Social cognitive theory: An Agentic Perspective. In: Annual Review of Psychology, 52, pp. 1-26.

Beetz, Tanja \& Eßer, Florian (2016): Kinder als Akteure- Forschungsbezogene Implikationen des erfolgreichen Agency Konzepts. In: Diskurs- Kindheits- und Jugendforschung, 11, 3, pp. 301-314.

Bourdieu, Pierre (1990): In Other Words: Essays toward a Reflexive Sociology. Cambridge, UK: Polity.

Bourdieu, Pierre (2001): Masculine Domination. Stanford: Stanford University Press.

Card, Noel, Stucky, Brian, Sawalani, Gita \& Little, Todd (2008): Direct and Indirect Aggression During Childhood and Adolescence: A Meta-Analytic Review of Gender Differences, Intercorrelations, and Relations to Maladjustment. In: Child Development 79,5, pp. 1185 - 1229.

Charmaz, Kathy (2014): Constructing Grounded Theory. Second Edition. Los Angeles: Sage.

Clarke, Adele (2003): Situational Analyses: Grounded Theory mapping after the postmodern turn. In: Symbolic Interaction 26, 4, pp. 553-576.

Danby, Susan J. \& Baker, Carolyn D. (1998): "What's the problem?": restoring social order in the preschool classroom. In: Hutchby, Ian \& Moran-Ellis, Jo (Eds.): Children and Social Competence: Arenas of Action. London: The Falmer Press, pp. 157-186.

Eberle, Scott G. (2014): The Elements of Play: Toward a Philosophy and Definition of Play. In: American Journal of Play 6, 2: 214-233

Edensor, Tim (2006): Reconsidering National Temporalities Institutional Times. Everyday Routines, Serial Spaces and Synchronicities. In: European Journal of Social Theory 9, 4, pp. 525-545.

Esen, Erol \& Taheri, Anahita (2016): Wissenschaftliche Diskussionen und Praktiken zur bilingualen frühkindlichen Bildung - Eine Einführung am Beispiel der 
Kinder und Jugendlichen mit türkischem Migrationshintergrund in Berlin. In: Esen, Erol (Hrsg.): Ein Kind - Zwei Sprachen. Bedingungen und Perspektiven der Deutsch-Türkischen Frühkindlichen Bildung am Beispiel Berlin. Ankara,Turkey: Siyasal Kitabevi-Ünal Sevindik, pp. 41-140.

Evans, Gary (2006): Child development and the physical environment. In: Annual Review of Psychology 57, pp. 423-51.

Fitzsimons Emla, Goodman, Alissa; Kelly, Elaine \& Smith, James P. (2017): Poverty dynamics and parental mental health: Determinants of childhood mental health in the UK. In: Social Science \& Medicine 175, pp. 43-51.

Flasher, Jack (1978): Adultism. In: Adolescence 13, 51: 517-523. Fontes, Lisa A. (2008): Interviewing Clients across Cultures: A Practitioner's Guide. New York: The Guilford Press.

Gogolin, Ingrid (2002): Linguistic and Cultural Diversity in Europe: a challenge for educational research and practice. In: European Educational Research Journal 1, 1, pp. 123-138.

Gogolin, Ingrid (1997): The "monolingual habitus" as the common feature in teaching in the language of the majority in different countries. In: Per Linguam 13,2 , pp. 38-49.

Huschke, Susann (2015): Giving Back: Activist Research with Undocumented Migrants in Berlin. In: Medical Anthropology 34, pp 54-69.

Kalkmann, Michael (2015): AIDA Country Report Germany. http://www.asylumineurope.org/news/24-08-2015/germany-halt-dublin-procedures-syrians.?country $=$ All\&topic $=$ All\&search $=$ \&page $=15$ (Accessed 18.06.2017).

Keller, Reiner (2013): Doing Discourse Research. London: Sage Publications Ltd. Laursen, Brett \& Collins, W. Andrew (1994): Interpersonal Conflict During Adolescence. In: Psychological Bulletin 115, 2, pp. 197-209.

Lefebvre, Henri (1991): The Production of Space. Oxford: Blackwell Publishers.

Lester, Stuart \& Russell, Wendy (2010): Children's right to Play: An examination of the importance of play in the lives of children worldwide. Den Haag: Bernard van Leer Foundation.

Lewek, Mirjam \& Naber, Adam (2017): Kindheit im Wartezustand. Studie zur Situation von Kindern und Jugendlichen in Flüchtlingsunterkünften in Deutschland. Köln: UNICEF.

Low, Setha \& Engle Merry, Sally (2010): Engaged Anthropology: Diversity and Dilemmas. In: Current Anthropology 51(S2), pp. S203-S226.

Mayall, Berry (2000): The sociology of childhood in relation to children's rights. In: The International Journal of Children's Rights 8, pp. 243-259.

Meire, Johan (2007): Qualitative research on children's play: a review of recent literature. In: Jambour, Tom\& van Gils, Jan (Eds.): Several Perspectives on Children's Play: Scientific Reflections for Practitioners. Antwerp: Garant, pp. 29-78.

Mouzourakis, Minos (2016) European Council on Refugees and Exiles, $\mathrm{http} / /$ eumigrationlawblog.eu/the-reception-of-asylum-seekers-in-europe-failing-common-standards/ (Accessed 12.08.2017). 
Parker, Jeffrey, Rubin, Kenneth, Erath, Stephen, Wojslawowicz, Julie \& Buskirk, Allison (2006): Peer Relationships, Child Development, and Adjustment: A Developmental Psychopathology Perspective. In: Cicchetti, Dante \& Cohen, Donald J. (Eds.): Developmental Psychopathology, Theory and Method. New Jersey: John Wiley \& Sons, pp. 419-493.

Pearlin, Leonard (1989): The sociological study of stress. In: Journal of Health and Social Behavior 30, pp. 241-256.

Prout, Allan \& James, Allison (1997): A new paradigm for the sociology of childhood? Provenance, promise and problems. In: James, Allison \& Prout, Alan (Eds.): Constructing and reconstructing childhood: Contemporary issues in the sociological study of childhood. London: Routledge Falmer, pp. 7-33.

Seeberg, Marie Louise, Bagge, Cecilie \& Enger, Truls André (2009): No place. Small children in Norwegian asylum-seeker reception centres. In: Childhood 16,3 , pp. 395-411.

Senatsverwaltung für Bildung, Jugend und Familie (2017) Kindertagesbetreuung: Bedarfsatlas. https://www.berlin.de/sen/jugend/familie-und-kinder/kindertagesbetreuung/fachinfo/\#kitaausbau (Accessed 20.06.2017)

Temple, Bogusia \& Edwards, Rosalind (2002): Interpreters/translators and crosslanguage research: reflexivity and border crossings. In: International Journal of Qualitative Methods 1, 2, pp. 1-12.

Vallotton, Claire, Harewood, Tamesha, Froyen, Laura, Brophy-Herb, Holly \& Ayoub, Catherine (2016): Child behavior problems: Mothers' and fathers' mental health matters today and tomorrow. In: Early Childhood Research Quarterly 37, pp. 81-93.

Wheaton, Blair, Young, Marissa, Montazer, Shirin \& Stuart-Lahman, Katie (2013): Social stress in the twenty-first century. In: Aneshensel, Carol, Phelan, Jo \& Bierman, Alex (Eds.): Handbook of the sociology of mental health (2nd ed.). New York, NY: Springer pp. 299-324.

Whitebread, David, Basilio, Marisol, Kuvalja, Martina \& Verma, Mohini (2012):

The importance of play: a report on the value of children's play with a series of policy recommendations. Brussels, Belgium: Toys Industries for Europe. 



\title{
„MANNO STOPP!“ Das Menschenrecht von jungen Kindern auf Schutz vor Gewalt in Unterkünften für geflüchtete Menschen
}

Evelyn Schulz-Algie

\section{Einleitung}

\begin{abstract}
„All I want is protection“ (Feldbesuch 4, GU 1, 5.7.2016), betont ein Vater, der mit seiner Frau und seiner Tochter aus dem Irak nach Deutschland geflohen ist, um Schutz für sich und seine Familie zu suchen. So wie dieser Familie geht es auch anderen Familien, die aufgrund von Krieg, Verfolgung und anderen massiven Gewaltbedrohungen und Menschenrechtsverletzungen ihre Heimat verlassen haben, um an einem anderen Ort in Frieden zu leben. Doch treffen sie in Deutschland auf die notwendigen Bedingungen, unter denen sie, und insbesondere ihre Kinder, ein gewaltfreies und friedvolles Leben führen können?
\end{abstract}

Alle Menschen haben das Recht auf Schutz vor jeder Form von Gewalt. In Bezug auf Kinder, die in der Bundesrepublik Deutschland leben, findet dieses Menschenrecht auf Schutz vor Gewalt seine rechtliche Verankerung sowohl in internationalen Menschenrechtsabkommen, unter anderem in der UN-Kinderrechtskonvention (UNKRK), als auch auf nationalstaatlicher Ebene. Dort ist der Bereich des Kinderschutzes, aufbauend auf dem Grundgesetz, vor allem im Sozialgesetzbuch VIII Kinder und Jugendhilfe sowie im Bürgerlichen Gesetzbuch (BGB) verankert. Für den Gewaltschutz von Kindern, die geflüchtet sind, ist zudem die rechtlich bindende EU-Aufnahmerichtlinie relevant. ${ }^{1}$

In diesem Beitrag gehe ich der Frage nach, inwieweit dem Menschenrecht von begleiteten jungen Kindern auf Schutz vor Gewalt in Unterkünften für geflüchtete Menschen gegenwärtig Rechnung getragen wird. Um dieser Frage näher auf den Grund zu gehen, werde ich folgende Forschungsfragen beleuchten: Mit welchen Formen von Gewalt sind Kinder in den Unterkünften konfrontiert? Wie gehen Mitarbeitende in Unterkünften mit Kinderschutz um?

1 Die Umsetzung der Richtlinie 2013/33/EU des Europäischen Parlaments und des Rates vom 26. Juni 2013 zur Festlegung von Normen für die Aufnahme von Personen, die internationalen Schutz beantragen (Neufassung), ist in der Bundesrepublik trotz der längst überfälligen Frist hierfür am 20. Juli 2015 bisher nicht erfolgt. 
Wie gehen Kinder mit Gewalt, mit der sie konfrontiert werden, um bzw. welche Ressourcen haben sie, um sich vor verschiedenen Formen von Gewalt zu schützen?

Auf der Suche nach Antworten bin ich während der Dateninterpretation immer wieder auf die Frage gestoßen, was Gewalt bedeutet und was demnach Kinderschutz vor Gewalt in dem spezifischen Kontext von Unterkünften für geflüchtete Menschen heißen kann. Eine Erläuterung des diesem Beitrag zugrunde liegenden Gewalt- und Kinderschutzverständnisses findet sich zusammen mit einer Beschreibung der methodologischen Herangehensweise im folgenden Kapitel.

Grundlage meiner Interpretation ist das Datenmaterial der von Scott und Le im Beitrag „It's child's play - or is it?“ und der von Scott im Beitrag „Entering the field as researchers and leaving as ,aunties " behandelten Erstaufnahmeeinrichtung (EAE), die aufgrund der Länge des Aufenthalts der Bewohner*innen faktisch als Gemeinschaftsunterkunft fungiert, sowie die beiden Gemeinschaftsunterkünfte, die im Zentrum des von Fichtner und Trần verfassten Beitrags „Handlungs-Spiel-Räume von Kindern in Gemeinschaftsunterkünften“" stehen. Zur Unterscheidung dieser beiden Gemeinschaftsunterkünfte ziehe ich die unterschiedlichen Größen heran und werde sie im Folgenden als die kleinere Gemeinschaftsunterkunft (GU 1) und die größere Gemeinschaftsunterkunft (GU 2) bezeichnen. Die Pseudonyme der Personen aus den Feldprotokollen verwende ich wie in den oben genannten Beiträgen.

Abschließend möchte ich darauf hinweisen, dass die hier dargestellten Ergebnisse keinen Anspruch auf Repräsentativität oder Vollständigkeit haben. Vielmehr eröffnen sie einen exemplarischen Einblick in die Praxis der Umsetzung des Kinderrechts auf Schutz vor Gewalt in Unterkünften für geflüchtete Menschen.

\section{Methodik}

Zwei Forscherinnentandems haben in drei unterschiedlichen Unterkünften Datenmaterial erhoben. Dieses Datenmaterial wurde von mir zunächst offen und dann aus einer Kinderrechtsperspektive mit Fokus auf Kinderschutz selektiv und axial kodiert, da sich zeigte, dass der Themenbereich Schutz von Kindern vor Gewalt im Datenmaterial aller Unterkünfte relevant ist. Im Kodierprozess bildeten sich drei Themenbereiche heraus, die sich in den drei Forschungsfragen widerspiegeln und in den folgenden drei Abschnitten wiederfinden.

In meiner Datenanalyse standen mir unterschiedliche Quellen zur Verfügung. In den Beobachtungsprotokollen der Feldforscherinnen finden sich sowohl Beobachtungsbeschreibungen aus der teilnehmenden Beobachtung der 
Forscherinnen als auch Beschreibungen der Feldforscherinnen von Berichten oder Erzählungen von Kindern, Eltern und Mitarbeiter*innen. Darüber hinaus lagen mir Interviewtranskriptionen vor, die von den Feldforscherinnen mit Mitarbeiter*innen oder Familienmitgliedern geführt, aufgezeichnet und anschließend transkribiert wurden. An dieser Stelle möchte ich darauf hinweisen, dass es sich bei den Datenquellen jeweils um Konstruktionen auf unterschiedlichen Ebenen handelt bzw. durch unterschiedliche Akteur*innen vorgenommen wurden. Während die für die Analyse herangezogenen Beobachtungsprotokolle bereits Resultate eines ersten Interpretationsschrittes der jeweiligen Feldforscherin und - wenn Erzählungen von Mitarbeiter*innen, Eltern und Kindern enthalten sind - auch deren Interpretationen sind, lassen die Interviewtranskriptionen eine unmittelbarere Interpretation durch mich zu. Die Unterschiedlichkeit der Datenquellen stellt eine methodische Herausforderung dar, da es sich um unterschiedliche Interpretationsebenen handelt. In der Darstellung meiner Ergebnisse habe ich die unterschiedlichen Datenquellen für die Leser*innen stets kenntlich gemacht und ihre Unterschiedlichkeit in meiner Interpretation berücksichtigt.

Die Tatsache, dass ich in meiner Analyse auf das Material angewiesen war, das die Forscherinnentandems im Feld erhoben haben, stellt eine Limitation meiner Ergebnisse dar. Da es Bestandteil des Forschungsdesigns und der Erhebungsmethode war, möglichst offen und unvoreingenommen ins Feld zu gehen, wurde in der Erhebung kein spezifischer Fokus auf das Recht auf Schutz vor Gewalt gelegt. ${ }^{2}$

Die Ergebnisse dieser Arbeit sind maßgeblich durch meine subjektive Perspektive auf das Datenmaterial aus dem Forschungsfeld geprägt, denn ,,jede Erkenntnis - auch die wissenschaftliche - trägt unweigerlich Merkmale des erkennenden Subjekts in sich, ist insofern unaufhebbar subjektiv - subjektgebunden, subjekthaft" (Breuer 2003: 2). Vor allem die Tatsache, dass dieser Beitrag aus der Perspektive einer weißen erwachsenen Frau aus der Mittelschicht geschrieben wurde, die in Deutschland aufgewachsen ist und keine Fluchterfahrung hat, ist als eine spezifische Perspektive zu betrachten. Um einer möglichen eingeschränkten Sichtweise entgegenzuwirken, wurde meine Interpretation im Austausch mit den einzelnen Forscherinnentandems, dem gesamten Forschungsteam sowie im Rahmen einer interdisziplinären Forschungswerkstatt mit Teilnehmer*innen außerhalb des Forschungsprojektes intersubjektiv validiert.

In der Dateninterpretation habe ich mich entsprechend eines induktiven Interpretationsvorgehens durch das Datenmaterial leiten lassen. Für die sozialen Phänomene, die ich aus dem Datenfundus herausgearbeitet habe, habe ich sowohl in dem Material selbst als auch durch Anknüpfen an theoretische Konzepte nach Erklärungsmöglichkeiten gesucht und somit mein induktives durch

2 Zur näheren Beschreibung der Erhebungsmethoden vgl. die Beiträge von Wihstutz „Das Forschungsprojekt und sein Design“, von Fichtner \& Trần und von Scott \& Le. 
ein deduktives Interpretationsvorgehen ergänzt. So halfen mir Konzepte von Gewalt und Kinderschutz aus der Literatur dabei, das Datenmaterial einzuordnen und mögliche Antworten auf meine Forschungsfragen zu finden. Gleichzeitig entwickelte ich aus dem Material heraus neue Zugänge und Ebenen im Zusammenhang mit meinen Forschungsfragen. Die in diesem Kapitel formulierten Konzepte und Kategorien sind somit Ergebnisse eines induktiv-deduktiven Analysevorgehens.

Um die Ergebnisse dieses Beitrags für die Leser*innen nachvollziehbar zu machen, werde ich im Folgenden auf die theoretischen Konzepte von Gewalt und Kinderschutz vor Gewalt eingehen. Eine Definition von Gewalt, die meinem Verständnis nahekommt, liefert Johan Galtung. Laut ihm liegt Gewalt vor, „wenn Menschen so beeinflusst werden, dass ihre tatsächliche körperliche und geistige Verwirklichung geringer ist als ihre mögliche Verwirklichung" (Galtung ohne Angabe zitiert nach Gugel 2006). Seine Einteilung von Gewalt in verschiedene Dimensionen, die in einer wechselseitigen Beziehung zueinander stehen, erweist sich als hilfreich für meine Interpretation des Datenmaterials. Laut Galtung liegt personale oder direkte Gewalt vor, wenn sowohl Täter*innen als auch Opfer der Gewalttat eindeutig identifizierbar und zuzuordnen sind (vgl. Galtung 1969: 170) und wenn die Gewalttat als konkrete Aktion sichtbar ist (vgl. ebd.: 171). Strukturelle Gewalt, die auch als indirekte Gewalt bezeichnet wird, bezieht sich auf Formen von Gewalt, die in gesellschaftlichen Strukturen verankert sind und sich als ungleiche Machtverteilung und folglich als ungleiche Lebenschancen äußern (vgl. ebd.). Unter dem Begriff der kulturellen Gewalt versteht Galtung kulturelle Phänomene wie Religion, Ideologie, Sprache, Kunst und Wissenschaft, die genutzt werden können, um personale oder strukturelle Gewalt zu legitimieren und Akzeptanz dafür in der Gesellschaft herzustellen (vgl. Galtung 1990: 291 f.). Neben Galtung wird in diesem Beitrag ferner auf das Gewaltverständnis der Weltgesundheitsorganisation (WHO) zurückgegriffen. Die WHO unterscheidet in ihrer Typologie mögliche Formen von Gewaltsituationen entsprechend der beteiligten Akteur*innen und unterteilt Gewalt nach physischer, sexueller und psychischer Gewalt und Vernachlässigung als Form der Gewalt (vgl. WHO 2003: 8). Neben diesen Formen führt die WHO auch das sogenannte Ökologische Modell zur Erklärung von Gewaltursachen ein. Unterschieden werden hier Einflussfaktoren auf der Ebene des Individuums, des Mikrosystems der Familie, der Nachbarschaft und Gemeinde und des Makrosystems der Gesellschaft, die in einer Wechselbeziehung zueinander stehen (vgl. WHO 2002: 12 f.). Diese unterschiedlichen Ebenen berücksichtigend, wird in einschlägiger Literatur zum Thema Kinderschutz auch häufig von Belastungsfaktoren (auch Risikofaktoren genannt) und Schutzfaktoren gesprochen, welche Studien zufolge die Wahrscheinlichkeit dafür, dass Kinder Gewalterfahrungen machen, möglicherweise erhöhen bzw. senken können (vgl. Albrecht; Deegener \& Körner; Egle; Fegert zitiert nach BVKJ 2016: 22 ff.). Das Konzept von Belastungs- und Schutzfaktoren, welche 
jedoch nicht als starr und absolut zu verstehen sind, hat diese Arbeit insofern bereichert, als dass ich in meiner Interpretation des Datenmaterials Faktoren identifizieren konnte, die den Schutz von Kindern vor Gewalt in bestimmten Situationen möglicherweise erhöhen oder verringern können. Darüber hinaus ist das diesem Beitrag zugrunde liegende Verständnis von Gewalt gegen Kinder maßgeblich durch Vertreter*innen aus dem Bereich der Kindheitssoziologie inspiriert, darunter Alanen (2005), Hengst \& Zeiher (2005), Honig (1999), James \& James (2008) und Johansson (2012). Diese Autor*innen beschäftigen sich unter anderem mit der Konstruktion von Kindheit und dem Konzept der generationalen Ordnung. Aufbauend auf diesen Überlegungen wird Gewalt gegen Kinder als eine Konsequenz aus dem auf der generationalen Ordnung basierenden Machtungleichgewicht zwischen Kindern und Erwachsenen angesehen, durch das sich Kinder in einer benachteiligten und vulnerablen Position wiederfinden (vgl. Finkelhor zitiert nach Alberth 2013: 68). ${ }^{3}$ „The abuse of power adults have over children" (Flasher 1978: 514) wird als Adultismus bezeichnet. ${ }^{4}$ Adultismus stellt eine Diskriminierungspraxis dar, die bei jeglicher Form von Gewalt, mit denen Kinder durch Erwachsene konfrontiert sind, mitschwingt. Gleichzeitig argumentiere ich in diesem Beitrag, dass ein adultistisches Verhalten von Erwachsenen gegenüber Kindern per se eine gewaltvolle Praxis darstellt, da fundamentale Rechte von Kindern, vor allem das Recht auf Partizipation, missachtet werden. Für das Verständnis des Schutzes von Kindern vor Gewalt ist daher ,die Machtkonstellation zwischen Erwachsenen und Kindern selbst in Frage zu stellen und den Kindern zu ermöglichen, auf gleicher Augenhöhe mit den Erwachsenen zu handeln“ (Liebel 2013: 94). Die UNKinderrechtskonvention, in der Kinder erstmals ausdrücklich als Rechtssubjekte mit eigenen und unveräußerlichen Grundrechten anerkannt werden (vgl. Maywald 2009: 5), ist eine zentrale Grundlage für den Gewaltschutz. So bietet der UN-Ausschuss für die Rechte des Kindes in seiner Allgemeinen Bemerkung Nr. 13 zum Recht des Kindes auf Schutz vor jeder Form von Gewalt (Artikel 19 UNKRK) zentrale Richtlinien für die Umsetzung dieses Menschen-

3 Dies soll nicht heißen, dass Kindern jegliches Handlungsvermögen entzogen ist; eine Vielzahl kindheitswissenschaftlicher Studien weist darauf hin, dass Kinder kompetente Akteur*innen ihres eigenen Lebens sind und Agency haben (vgl. Corsaro zitiert nach Alberth 2013: 69). Nichtsdestotrotz führt die ungleiche Machtverteilung häufig dazu, dass das Handlungsvermögen von Kindern eingeschränkt ist, denn „Handlungsvermögen ist [...] untrennbar mit der ,Macht‘ (oder deren Fehlen) verbunden“" (Alanen 2005: 80).

4 Laut Bell (1995: 1) basiert Adultismus ,on the assumption that adults are better than young people, and entitled to act upon young people without their agreement". 
rechts. ${ }^{5}$ Demnach hat der Staat als wichtigster „Duty Bearer“6 die Pflicht, die in der UNKRK verankerten Menschenrechte für Kinder sicherzustellen. Hierbei sind insbesondere die ,general principles“ (allgemeinen Prinzipien) der UNKRK - das Recht auf Nichtdiskriminierung (Artikel 2 UNKRK), das Recht auf Berücksichtigung des Wohles des Kindes (Artikel $3 \S 1$ UNKRK), das Recht auf Leben, Überleben und Entwicklung (Artikel 6 UNKRK) und das Recht auf Berücksichtigung der Meinung des Kindes (Artikel 12 UNKRK) Grundprinzipien zur Auslegung und Implementierung des Menschenrechts auf Schutz vor Gewalt (vgl. United Nations 2011: 34 f.). In diesem Sinne wird Kinderschutz in dieser Arbeit als die Umsetzung des Menschenrechts von Kindern auf Schutz vor Gewalt angesehen, wobei das Gewalt- und Kinderschutzverständnis durch die oben aufgeführte Literatur inspiriert und in meiner Interpretation des Datenmaterials angepasst und weiterentwickelt wurde.

\section{Dimensionen von Gewalt gegen Kinder in den Unterkünften}

Im Folgenden werde ich auf Grundlage der empirischen Daten Dimensionen ${ }^{7}$ von Gewalt, denen Kinder in den drei Unterkünften für geflüchtete Menschen ausgesetzt sind, herausarbeiten und analysieren. Hinweisen möchte ich an dieser Stelle darauf, dass ich lediglich die Gewaltformen thematisiere, die sich mir durch die Analyse des Datenmaterials aufgezeigt haben. Gewaltformen, auf die es im Material keine Hinweise gab, werde ich vernachlässigen - ohne damit aussagen zu wollen, dass sie in den Forschungskontexten nicht existieren.

Das Datenmaterial zeichnet ein Bild von Lebensverhältnissen in den Unterkünften, in denen fundamentale Rechte von Kindern in vielerlei Hinsicht verletzt werden. Sie sind geprägt von sozialer Exklusion, Ressourcenknappheit, mangelnder Privatsphäre, mangelnder Ruhe und mangelnden Rückzugsmöglichkeiten, unhygienischen Zuständen der sanitären Anlagen und

5 Neben vielen Bezügen zum Kinderschutz stellt vor allem das Recht auf Schutz vor Gewaltanwendung, Misshandlung und Verwahrlosung (Artikel 19) eine „Kernbestimmung für Diskussionen und Strategien für den Umgang mit und die Abschaffung von jeder Form von Gewalt" (United Nations 2011: 8) dar.

6 Der Staat, der die UNKRK ratifiziert hat, wird in der Konvention als wichtigster ,duty bearer" (Verantwortungsträger) angesehen. Durch die Ratifizierung verpflichtet er sich, dafür zu sorgen, dass Kinder die in der Konvention verbrieften Rechte wahrnehmen können.

7 Die Begriffe Formen und Dimensionen von Gewalt werden in diesem Beitrag als Synonyme verwendet. 
unzureichenden Bedingungen der Essensversorgung. Diese und weitere Faktoren, die durch die Rahmenbedingungen von Unterkünften geprägt sind, stellen strukturelle Formen von Gewalt dar. Der Staat, der durch die Einrichtung und Aufrechterhaltung diese gewaltvollen Unterbringungsformen und -bedingungen maßgeblich verantwortet, kommt somit nicht nur seiner menschenrechtlich verankerten Pflicht, den Schutz von Kindern vor jeglicher Form von Gewalt sicherzustellen, nicht nach, sondern verletzt selbst elementare Menschenrechte. Dies gilt nicht nur für die Rahmenbedingungen in den Unterkünften, sondern für das gesamte Asylsystem, mit dem die asylsuchenden Familien in der Bundesrepublik konfrontiert werden (siehe den Beitrag von Wihstutz „Mittendrin und außen vor"). Vor allem die mit einer Duldung verbundenen sozialen und wirtschaftlichen Benachteiligungen sowie die (drohende) Abschiebung von Menschen stellen äußerst gewaltvolle Praxen dar, wie das Datenmaterial eindrücklich nahelegt. Beispielhaft soll hier die Situation des Mädchens Lara, bei der frühkindlicher Autismus diagnostiziert wurde, genannt werden. Sie wurde während unserer Forschung mit ihren Eltern in ihr Heimatland abgeschoben. Während Lara laut ihrer Mutter, der Kinderbetreuerin sowie den Beobachtungen der Forscherinnen während ihres Aufenthalts in Berlin zufolge erstaunliche Fortschritte (Feldbesuch 9, GU 1, 10.8.2016) gemacht habe, immer kontaktfreudiger und kommunikativer wurde, beschreibt die Mutter nach der Abschiebung in ihr Heimatland in einer E-Mail an die Forscherinnen, dass es Lara sehr schlecht gehe. Ihre Tochter sei verwirrt und verloren (E-Mail-Korrespondenz, GU 1, 21.9.2016), habe ihre Routine und ihre Sicherheit verloren, habe sich in sich zurückgezogen, sei unruhig, schreie viel und schlafe wenig (vgl. ebd.). Von den Behörden und der Stadt bekomme sie keine Hilfe: [H]ier [in ihrem Heimatland, Anm. d. Verf.] sind Kinder nicht geschützt, es gibt auch keine qualifizierten Ärzte, die sich mit Autismus befassen, es liegt alles an mir (ebd.). Die Abschiebung der Familie führte also dazu, dass Lara in ihrem Heimatland fundamentale Rechte verwehrt bleiben; das Kindeswohl wird bei der Entscheidung zur Abschiebung nicht berücksichtigt, ebenso wenig die in der UN-Kinderrechtskonvention in Artikel 23 explizit für Kinder mit Behinderung verankerten Rechte.

Die Gewaltformen, die von Mitarbeitenden der drei Einrichtungen am häufigsten angesprochen oder geschildert werden, sind Formen innerfamiliärer Gewalt. So berichtet die Heimleitung der großen Gemeinschaftsunterkunft von einer Familie, in der der Vater seiner Frau gegenüber körperlich gewalttätig geworden sei (vgl. Interview bei dem Feldbesuch 10, GU 2, 20.5.2017). In einem Beobachtungsprotokoll der Erstaufnahmeeinrichtung erwähnt ein Mitarbeiter bezüglich eines Mädchens, das sowohl den Kinderbetreuer*innen als auch den Forscherinnen durch gewaltvolle Konflikte mit anderen Kindern auffiel, that he thought that Farah was being hit at home (Feldbesuch 10, EAE, 18.7.2016). Auch eine Mutter aus der kleineren Gemeinschaftsunterkunft erzählt, dass sie oftmals mitbekommen habe, dass eine Familie, die zum 
Zeitpunkt der Forschung anscheinend nicht mehr in der Unterkunft lebte, ständig Streit gehabt hatte. [...] Sie meinte dazu, dass die Kinder ,,immer geschlagen" wurden und sie sie ständig weinen hörte (Feldbesuch 5, GU 1, 15.7.2016). Die Forscherinnen konnten keine Situationen innerfamiliärer Gewalt beobachten.

Was die Forscherinnen beobachten bzw. erfahren, ist, dass der hohe Grad an Öffentlichkeit und die mangelnden Rückzugsräume in den Unterkünften zu einem erhöhten Stresspegel bei den Menschen, die in den Unterkünften leben und arbeiten - und auch forschen - führen kann. Wie fließend die Grenzen zwischen Öffentlichkeit und Privatheit in den Unterkünften sind, erleben die Forscherinnen in der kleineren Gemeinschaftsunterkunft unter anderem bei ihrem Rundgang mit einem Hausmeister durch die Unterkunft. Er führt sie - zum Unbehagen der Forscherinnen -, ohne vorher anzuklopfen und um Eintritt zu bitten, in ein Zimmer, dessen Türen offen stehen und in dem vier Männer wohnen, von denen zwei gerade anwesend sind. Die Forscherin notiert hierzu folgende Gedanken in ihr Beobachtungsprotokoll:

Mir war es unangenehm und ich war auch irgendwie sauer auf mich. Mir wurde klar, dass es sich gerade um den Raum von den Bewohnern handelte und nur, weil eine Tür offen stand, das keine Einladung war, in die Privatsphäre einzudringen oder auch der freundlichen Einladung des Hausmeisters Folge zu leisten (Feldbesuch 10, GU 1, 24.8.2016).

Unabhängig davon, dass die fehlende Privatsphäre eine Form struktureller Gewalt darstellt, kann dieser Kontext die Familiendynamik negativ beeinflussen. In diesem Zusammenhang hält eine Forscherin aus der Erstaufnahmeeinrichtung in einem Beobachtungsprotokoll fest: They all find it difficult to live together in 1 room that is only 14 square meters in size (Feldbesuch 18, EAE, 5.10.2016). Daneben können sich auch andere restriktive Faktoren, die sich beispielsweise aus einer fehlenden Aufenthaltserlaubnis ergeben, negativ auf die Familiendynamik auswirken. So berichtet die Mutter einer Familie, die mit einer Duldung in der größeren Gemeinschaftsunterkunft lebt: Alles ist von der Aufenthaltserlaubnis abhängig. Man kann überhaupt nichts machen, ohne einen Aufenthaltstitel zu bekommen oder zu haben (Feldbesuch 10, GU 2, 20.5.2017). Der Vater der Familie fügt hinzu, dass ihn die Situation, keiner Erwerbstätigkeit nachgehen zu können, sehr belaste und sich auch negativ auf seinen Umgang mit seinem Sohn Spiderman auswirke. Es tut mir nicht gut und macht der ganzen Familie Probleme. Deshalb sagt Spiderman auch, dass ich ärgerlich sei (ebd.). Die Mutter ergänzt: Er spricht manchmal zu laut mit Spiderman (Feldbesuch 8, GU 2, 11.2.2017).

Eine andere Situationsbeobachtung, die auf den Zusammenhang der herrschenden Bedingungen in der Unterkunft und von innerfamiliären Konflikten hindeutet, findet sich in den Beobachtungsprotokollen der Forscherinnen in der Erstaufnahmeeinrichtung. Nachdem die Forscherin mit Kindern aus der 
kleineren Gemeinschaftsunterkunft gemalt hat, bemerkt ein Mädchen, dass es rote Farbe auf seiner Kleidung habe. Die Forscherin notiert in ihrem Beobachtungsprotokoll:

Zarin, the 8 year old, suddenly got really distressed when she realised there were red felt marker stains on her dress. She started to cry loudly, tears rolling down her cheeks while she said something in Farsi. Ms. Sanjari [eine anwesende Mutter] [...] said something to me in Farsi and I heard the word „Mutter". I looked at her and repeated the word ",Mutter" and slapped the back of my hand to indicate that the mother might be angry with her because of the stains on her clothes. Ms. Sanjari shook her head in agreement. I wondered if this might be the reason for the child's anxiety because the washing facilities are a source of discontent among the residents; the child's mother may have instructed her to try and keep her clothes as clean as possible as a result. This was one possible theory. [...] I took Zarin to the bathroom. [...] I was really surprised at how anxious the child was and I wondered what lay behind it. She kept inspecting the area I was rubbing to see if the stains were out. If she noticed even the slightest hint of red, she insisted that I rub it again (Feldbesuch 6, EAE, 1.7.2016).

Zwar handelt es sich bei der hier beschriebenen Interpretation der Forscherin auf die Reaktion des Mädchens Zarin auf den Farbfleck auf ihrer Kleidung nur um eine Vermutung. Doch tatsächlich könnte es einen Zusammenhang zwischen der beobachteten Reaktion von Zarin und den Bedingungen in der Unterkunft geben. So erfährt eine Forscherin über eine Unterhaltung zwischen Eltern: there were also complaints about the fact they had to wait 2 months for a slot to use the washing machines (Feldbesuch 5, EAE, 22.6.2016). Dies deckt sich mit dem, was die Heimleitung über den Mangel an Waschmaschinen erzählt, sowie mit der Beobachtung der Forscherinnen von langen Warteschlangen der Bewohner*innen vor dem Waschraum (vgl. Feldbesuch 1, EAE, 2.6.2016 und Feldbesuch 5, EAE, 23.6.2016). Nach der hier beschriebenen Interpretation der Situation ließe sich ein Zusammenhang zwischen der Knappheit an Waschmöglichkeiten für Bewohner*innen in der Unterkunft und der von der Forscherin beschriebenen Reaktion von Zarin auf den Farbfleck auf ihrer Kleidung ziehen, welche wiederum mit möglichen Erwartungen ihrer Mutter an sie, die Kleidung sauber zu halten, und einer möglicherweise für Zarin unangenehmen Reaktion der Mutter auf einen Farbfleck in der Kleidung in Verbindung gebracht werden kann.

Darüber hinaus gibt es Hinweise dafür, dass sich Eltern aufgrund von Sorgen und psychischen Belastungen wie traumatischen Erlebnissen nicht in der Lage fühlen, die Wünsche und Bedürfnisse ihrer Kinder so zu erfüllen, wie sie es gerne tun würden. Eine Mutter erzählt in diesem Zusammenhang, dass ihre Tochter Farah gerne mit ihr spielen würde, sie jedoch keine Kraft und keine Lust dazu habe. Sie habe so viele Sorgen (vgl. Interview bei dem Feldbesuch 18, EAE, 5.10.2016). In einem Interview mit ihr erzählt die Mutter, dass sie 
Angst habe, wenn Farah alleine in der Einrichtung oder auf dem Gelände der Unterkunft unterwegs sei. Sie bitte ihre Tochter beispielsweise, nicht in den Park zu gehen, und erzählt: [I]ch habe Angst, dass alle Leute, alle Leute so streiten und dass etwas passiert. [...] Ich habe immer Angst, wenn Farah draußen ist (vgl. ebd). Auch habe sie Angst, wenn Farah nachts alleine auf die Toilette im Flur der Unterkunft gehe, weil es sind alles gemischte Leute [...]. Besonderes für Frauen ist es schwierig (vgl. ebd.). Farah selbst habe auch Angst davor, doch da die Mutter krank sei, gehe Farah alleine auf Toilette (vgl. ebd.). Insgesamt unterstreicht die Situation dieser Familie, dass die Bedingungen in der Unterkunft für Familien, die in schwierigen Lebenssituationen leben, gänzlich ungeeignet sind und vielfache Risiken für Kinder bergen, weitere Gewalterfahrungen zu machen.

In Gesprächen mit Mitarbeiter*innen der größeren Gemeinschaftsunterkunft wird das Thema der Aufsichtspflicht von Eltern von den Mitarbeitenden wiederholt angesprochen, häufig in Verbindung mit der Einschätzung einer mangelnden Wahrnehmung der Aufsichtspflicht der Eltern. Es geht vor allem um die Aufsichtspflicht. Das ist wirklich ein großes Thema bei uns, berichtet eine Mitarbeiterin. So seien Kinder oft unbeaufsichtigt von ihren Eltern in den Räumlichkeiten der Einrichtung und teilweise auch außerhalb des Gebäudes unterwegs. Teilweise verwenden Mitarbeitende der Unterkünfte in diesem $\mathrm{Zu}-$ sammenhang auch den Begriff der Vernachlässigung der Kinder durch ihre Eltern.

Die Heimleiterin berichtete wiederholt von dem Vorwurf der Kindervernachlässigung durch die Eltern und adressierte das Anliegen an uns, da mal ,,in die Tiefe zu schauen ", um zu verstehen, ob die Eltern nicht anders können, ob es an einem anderen Erziehungsverständnis liege oder sie einfach keine Kraft hätten (Feldbesuch 1, GU 2, 9.12.2016).

Die Forscherin lässt eine Kinderbetreuerin der Unterkunft sprechen:

[...] dass die Bewohner sich in dem Haus wie in einem Dorf fühlten, in dem Kinder frei herumlaufen. Aus Kinderschutzperspektive sei das gefährlich: „,Wenn einer ein Kind mit Schokolade in ein Zimmer lockt, weiß man nicht, was passiert“. „, Oder wenn ältere Geschwister auf die Kleinen aufpassen oder wenn die Eltern nicht auffindbar sind nach der Kinderbetreuung und man die Kleinen bei den Nachbarn, abgeben ' muss ... Das sind dann auch Situationen, in denen man auch sprachlich nicht weiterkommt. "Sie erzählte von einem konkreten Fall eines dreizehnjährigen Kindes, das ihr kleines Geschwisterkind zum Aufpassen in eine andere Familie gegeben habe (ebd.).

Auf die Frage, ob es sich bei den geschilderten Situationen tatsächlich um Situationen handle, in denen Eltern ihre Kinder vernachlässigten, kann und möchte ich aufgrund der dünnen Informationslage an dieser Stelle nicht eingehen. Vor allem die Perspektive der jeweiligen Eltern und Kinder auf die geschilderten Situationen liegen nicht vor und wären für eine Analyse der 
Situation wichtig. Allerdings werfen die Zitate in mir die Frage auf, wer in einer Unterkunft entscheidet, ob und wann sich Kinder alleine in oder außerhalb der Unterkunft bewegen dürfen. Welche Verantwortung haben hierbei neben den Eltern die Mitarbeiter*innen und ab wann gilt die Aufsichtspflicht der Eltern in einer Unterkunft, in der die Grenzen zwischen privatem und öffentlichem Raum so fließend sind, als verletzt?

Das Thema der Vernachlässigung von Kindern durch ihre Eltern wird auch im Datenmaterial der Erstaufnahmeeinrichtung angesprochen. Konkret gibt es in einem Beobachtungsprotokoll eine Situationsbeschreibung, in der eine Mitarbeiterin in Bezug auf zwei Mädchen, die mit ihrem Vater in der Unterkunft leben, berichtet,

how difficult their home situation is. She said that when she sees them she tries to talk with them and give them a hug because they ,brauchen Liebe und Aufmerksamkeit". A social worker said that they are often neglected by their father and when she can, she sometimes gives them things they need or helps out where she can. She spoke with considerably emotion about their situation and mentioned repeatedly the father's inability to take care of the children. She mentioned that he has to bathe them and that must be difficult for him. She claimed there have been times that the eldest child has not gone to school because the father is asleep and so was not able to get her up and ready to leave for classes. [...] She replied that he is angry that he has only received subsidiary protection and he has said ,er braucht nur eine Wohnung und dann wird es ihm besser gehen "(Feldbesuch 21, EAE, 7.12.2016).

Auch an dieser Stelle kann und möchte ich keinerlei Bewertung für das Vorliegen einer Vernachlässigung machen. Hervorheben möchte ich jedoch den Punkt, dass sowohl die beschriebene Unzufriedenheit des Vaters mit seinem Aufenthaltsstatus als auch seine Aussage, er brauche eine Wohnung, damit es ihm besser gehe, in meinen Augen auf strukturelle Formen von Gewalt hinweisen, denen der Vater und seine Familie durch das Asylsystem ausgesetzt sind. Inwieweit diese von mir als Gewalt interpretierten Erfahrungen des Vaters tatsächlich auf seine Fähigkeit der Wahrnehmung seiner Erziehungsverantwortung Einfluss nehmen - über die wir über die Bewertung einer Mitarbeiterin hinaus keine weiteren Hinweise haben -, bedürfte einer tieferen Analyse. Dennoch weist diese Situation darauf hin, dass Gewalt, die in Strukturen wie dem Asylsystem verankert ist, Auswirkungen auf Dynamiken innerhalb von Familien haben können. Ein weiterer Punkt, der mir auffällt, ist, dass die Mitarbeiterin den Vater in der Wahrnehmung seiner Erziehungsverantwortung als unfähig beschreibt, während mögliche Stärken oder Ressourcen seinerseits nicht angesprochen werden. Während die Mitarbeiterin offenbar versucht, den Kindern „Liebe und Aufmerksamkeit“ zu schenken, bleibt unklar, inwieweit es Bemühungen seitens der Mitarbeitenden gibt, den Vater in seiner Erziehungsverantwortung zu stärken und hierfür auch die vorhandenen Ressourcen und Bedürfnisse der Familie in den Blick zu nehmen. Die Haltung von 
Mitarbeiter*innen gegenüber in Unterkünften lebenden Menschen ist angesichts der Tatsache, dass diese in einem Abhängigkeitsverhältnis zum Unterkunftspersonal stehen, umso bedeutender für die Familien. Insgesamt verdeutlicht die Situation, wie machtlos Menschen durch strukturelle Formen von Gewalt im Rahmen des Asylsystems gemacht werden und wie ihnen dadurch ein selbstbestimmtes Leben erschwert wird.

Eine weitere Ebene der Konfrontation von Kindern mit Gewalt im familiären Umfeld, die ich nicht unerwähnt lassen möchte, ist, wenn Kinder die Erzählungen der Eltern von Gewalterfahrungen mithören. So wird in einem Beobachtungsprotokoll aus der kleineren Gemeinschaftsunterkunft geschildert, wie der Vater den Forscherinnen in der Anwesenheit seiner Tochter - die sich spielend mit einer der Forscherinnen beschäftigt - von seinen Gewalterfahrungen in einem Drittland sowie von seinen Gefühlen bezüglich seines Abschiebebescheids erzählt (vgl. Feldbesuch 4, GU 1, 5.7.2016 und Feldbesuch 6, GU 1, 21.7.2016). In einem Beobachtungsprotokoll aus der größeren Gemeinschaftsunterkunft wird beschrieben, wie der Vater einer Familie vom Tod seiner Schwester im Krieg berichtet, und die Mutter erzählt von gewaltvollen Erlebnissen, die ihr Sohn im Krieg im Heimatland mitbekommen hat (vgl. Feldbesuch 8, GU 2, 11.2.2017). Der Junge hält sich während des Gesprächs mit im Zimmer auf - tatsächlich bietet der private Raum der Familie auch keine weiteren Rückzugsmöglichkeiten. Betonen möchte ich, dass ich die Entscheidung der Eltern, über die Erfahrungen und Erlebnisse in der Anwesenheit ihrer Kinder zu sprechen, keinesfalls be- oder verurteilen möchte. Vielmehr geht es mir darum aufzuzeigen, in welchen Situationen Kinder auf verschiedene Weise mit Gewalterfahrungen in Berührung kommen. ${ }^{8}$

In diesem Zusammenhang möchte ich auch selbstbezogene Gewaltgedanken von Familienmitgliedern erwähnen. Aus dem Datenmaterial geht zwar nicht hervor, ob die Kinder von diesen Gedanken wissen, es kann jedoch angenommen werden, dass sie insbesondere bei einer engen Eltern-Kind-Bindung spüren, wie es ihren Eltern geht. Beispielsweise erzählt eine Mutter aus der Erstaufnahmeeinrichtung, that there were times that she felt she wanted to die because of the situation (Feldbesuch 18, EAE, 5.10.2016), und ein Vater aus der kleineren Gemeinschaftsunterkunft äußert: I feel sometimes like I want to kill myself (Feldbesuch 4, GU 1, 5.7.2016).

Eine weitere Dimension von Gewalterfahrungen, die Kinder in den Unterkünften machen, sind gewaltvolle Konflikte zwischen Kindern. Hierauf weisen

Diese Situation kann be- und entlastend sein. Eine wichtige Rolle spielt dabei, inwiefern es bei den Beteiligten (Erwachsenen) eine Wahrnehmung dafür gibt, welche Wirkung die Situation auf sie und auf die Kinder hat. Die Sequenz zeigt, wie wichtig es ist, dass Mitarbeiter*innen in den Unterkünften über Wissen in den Bereichen der Psychotraumatologie und Traumapädagogik verfügen und die Bewohner*innen sich in Begleitung ausgebildeter Menschen mit ihren traumatischen Erlebnissen auseinandersetzen können. 
sowohl Mitarbeiter*innen der Einrichtungen als auch Beobachtungen der Feldforscherinnen in der Erstaufnahmeeinrichtung und in der großen Gemeinschaftsunterkunft hin, die sich sowohl in körperlichen als auch in verbalen Auseinandersetzungen widerspiegeln. Insgesamt ist auffällig, dass in der Erstaufnahmeeinrichtung, in der die materielle und personelle Ressourcenknappheit besonders heraussticht, Konflikte zwischen Kindern häufiger beobachtet werden. Ein Beispiel eines gewaltvollen Konflikts zwischen Kindern stellt folgendes Zitat aus einem Beobachtungsprotokoll der Erstaufnahmeeinrichtung dar:

Taban was playing with a plastic watering can when Farah grabbed it from her and she started crying. I told Farah (in German) to give it back, which she did and the children resumed playing. Suddenly I heard a child shouting and I looked over to see Farah hit Taban on her forehead. Taban fell backward and hit her head on a toy dolly stroller. She started howling and held her forehead (Feldbesuch 6, EAE, 1.7.2016).9

Darüber hinaus berichten Mitarbeiter*innen aus der Erstaufnahmeeinrichtung und der großen Gemeinschaftsunterkunft auch von rassistischen Äußerungen und Handlungen unter Kindern, wobei es im Datenmaterial keine weiteren Hinweise dazu gibt.

Auch bietet das Datenmaterial Situationsbeschreibungen, in denen einige Mitarbeitende einen Umgang mit Kindern pflegen, der als gewaltvoll und adultistisch interpretiert werden kann und sich unter anderem im Anschreien oder Beschimpfen von Kindern äußert bzw. als autoritär bezeichnet werden kann. So wird in einem Beobachtungsprotokoll aus der Erstaufnahmeeinrichtung beschrieben, dass eine mitarbeitende Person der Kinderbetreuung die Kinder häufig anschreie oder in einem bossy tone (Feldbesuch 4, EAE, 16.6.2016) mit ihnen spreche, wenn sie ihnen Anweisungen gebe oder sie ermahne (siehe den Beitrag von Scott \& Le). In der größeren Gemeinschaftsunterkunft protokollieren die Forscherinnen ebenfalls, dass ein Kinderbetreuer eher etwas rauer und lauter gegenüber den Kindern (Feldbesuch 6, GU 2, 26.1.2017) sei. Da sich eine Gruppe von Kindern bei der Heimleitung über den betreffenden Mitarbeiter beschwert, kann angenommen werden, dass die Kinder seinen Umgang mit ihnen als gewaltvoll und nicht angemessen empfinden. Das Datenmaterial zu diesen Situationen weist darauf hin, dass die betreffenden mitarbeitenden Personen im Umgang mit den Kindern, gerade wenn es um Regelverstöße seitens der Kinder geht, überfordert sind und auch insgesamt auf die Forscherinnen gestresst (Feldbesuch 3, GU 2, 21.12.2016) wirken. Daneben finden sich in den Beobachtungsprotokollen Situationsbeschreibungen, in denen Mitarbeitende die körperliche Distanz offenbar gegen den Willen von

9 Die Thematik von Konflikten zwischen Kindern und eine Auseinandersetzung mit möglichen (strukturellen) Zusammenhängen dieser Konflikte wird im Beitrag von Scott und Le tiefergehend bearbeitet. 
Kindern überschreiten. So werden in den Protokollen der kleineren Gemeinschaftsunterkunft zwei Situationen beschrieben, in denen eine technische Hilfskraft dem Mädchen Elsa körperlich nahekommt, obwohl sie ihm mehrfach signalisiert, dass sie Distanz wahren möchte. (Die beiden Situationen werden weiter unten ausführlich behandelt.) In diesen Situationen schwingt in meinen Augen das Spannungsverhältnis zwischen einem spielerischen Umgang und einem übergriffigen Verhalten von Mitarbeitenden mit Kindern mit.

Auch mit solchen Formen von Gewalt, bei denen Kinder zwar nicht direkt betroffen sind, Gewaltsituationen jedoch in ihrem unmittelbaren Umfeld auftreten, sind Kinder konfrontiert. Ein Beispiel hierfür ist ein Vorfall einer Messerstecherei zwischen erwachsenen Bewohner*innen in der größeren Gemeinschaftsunterkunft (vgl. Feldbesuch 10, GU 2, 20.5.2017).

Abschließend möchte ich thematisieren, dass auch unsere Forschung, die Teil des Feldes geworden ist, nicht von der (Re-)Produktion von Gewalt ausgenommen ist (siehe hierzu auch den Beitrag von Trần). So gibt es im Datenmaterial der Erstaufnahmeeinrichtung Hinweise dafür, dass das Konkurrieren um die Aufmerksamkeit der anwesenden Forscherinnen unter Kindern zu Konflikten führen kann. Die Aussage einer Kinderbetreuerin unterstützt diese Annahme: Es sei bestimmt toll für das Mädchen, wenn sie [durch die Forschung] eins zu eins Aufmerksamkeit bekäme. Ihre einzige Möglichkeit auf sich aufmerksam zu machen sei momentan, andere Kinder zu schlagen und das gefiele den anderen Kindern nicht (Feldbesuch 9, EAE, 18.7.2016). Scott analysiert in ihrem Beitrag eine entsprechende Situation aus ihrer teilnehmenden Beobachtung und stellt eine Verbindung zur Knappheit an sozialen Ressourcen her, mit denen Kinder in der Unterkunft offenbar konfrontiert sind. Die Beschreibung einer weiteren Situation, welche von den beteiligten Kindern möglicherweise als gewaltvoll empfunden worden sein kann, findet sich in einem Beobachtungsprotokoll aus der kleineren Gemeinschaftsunterkunft. Damit die Forscherinnen mit Lara, die von ihrer Mutter als autistisch beschrieben wird, ungestört in dem Kinderbetreuungsraum forschen können, wird die Kinderbetreuung für diesen Zeitraum ausgesetzt. Der Situation voraus geht eine entsprechende Vereinbarung zwischen den Forscherinnen und einer Kinderbetreuerin, da Lara die Anwesenheit anderer Kinder laut ihrer Mutter zu viel sei. Die beiden Jungen, die sich im Kinderbetreuungsraum befinden, wollen jedoch nicht gehen, was sie verbal sowie durch Weglaufen vor der Kinderbetreuerin äußern. Erst das Versperren des Weges der Kinder durch eine Forscherin, wozu sie durch die Kinderbetreuerin aufgefordert wurde, bewegt die beiden dazu, den Raum zu verlassen, woraufhin die Kinderbetreuerin den Jungen ankündigt, mit ihren Eltern über ihr Verhalten zu sprechen. Diese Situation kann als Ausgrenzung der Jungen ohne jegliche Möglichkeit der Mitbestimmung interpretiert werden, die durch unsere Forschung verursacht wurde. Gleichzeitig möchte ich hervorheben, dass Lara tatsächlich erst durch die beiden Forscherinnen $\mathrm{Zu}-$ gang zu dem Kinderbetreuungsraum erhalten hat und die Ausgrenzung der 
Jungen demnach mit der Schaffung des Zugangs von Lara zu dem Kinderbetreuungsraum einhergeht. Diese Ambivalenz der (Forschungs-)Intervention in diesem Feld kann als ein Dilemma beschrieben werden, von dem die Forschung nicht ausgenommen ist. Über diese Beispiele hinaus müssen wir Forscherinnen uns auch die Frage stellen, inwieweit das Beobachten bzw. Wahrnehmen von Gewalt, ohne einzugreifen, zur Tolerierung von Gewalt beiträgt.

Insgesamt zeigt die Analyse des Datenmaterials, dass die Dimensionen von Gewalt, mit denen Kinder in den Unterkünften konfrontiert sind, äußerst vielfältig, komplex und ineinander verschränkt sind. Vor allem wird deutlich, dass die vielfältigen Formen struktureller Gewalt einen Nährboden für weitere gewaltvolle Praxen darstellen und eng mit anderen Dimensionen von Gewalt verwoben sind.

\section{Umgang mit Kinderschutz in den Unterkünften}

In diesem Kapitel werde ich anhand von einigen Schlüsselszenen aus den Beobachtungsprotokollen der Forscherinnentandems und aus Interviewtranskripitionen exemplarisch aufzeigen, wie seitens der Mitarbeiter*innen mit Kinderschutz vor Gewalt in den Unterkünften umgegangen wird.

Die Analyse des Datenmaterials zeigt, dass es eine Kluft zwischen aufgestellten Kinderschutzregelungen oder -vorstellungen auf der einen Seite und der gelebten Praxis auf der anderen Seite gibt. In der Erstaufnahmeeinrichtung beispielsweise schreibt eine Forscherin in ihrem Beobachtungsprotokoll: I asked the child care manager what rules I should be mindful of when I was in the Kinderzimmer. She responded by saying firstly, that I won't ever be alone in there with the kids without a Mitarbeiter (Feldbesuch 4, EAE, 16.6.2016). Zwar geht aus dem Feldprotokoll nicht hervor, auf welcher Begründung die Regel basiert, doch kann vermutet werden, dass die Regel einem möglichen Fehlverhalten oder einem möglichen Missbrauch einer erwachsenen Person, die nicht zum festen Personal gehört, in der Kinderbetreuung entgegenwirken soll, wie es in vielen Einrichtungen, die mit Kindern arbeiten, oft die Regel ist. Offen bleibt, ob die Regel auch ohne das Erfragen der Forscherin an sie kommuniziert worden wäre, also ob diese Regel generell an alle Personen von auBen kommuniziert wird oder nicht. Es ist daher unklar, ob und wie fest diese Regel institutionell verankert ist. Die Forscherin protokolliert anschließend, dass sie sich mehrere Male mit den Kindern allein in der Kinderbetreuung wiederfinde, da die Kinderbetreuer*innen zu anderen Aufgaben abberufen würden. Zudem werden immer wieder Situationen beobachtet, in denen sich die Kinder unbeaufsichtigt in unmittelbarer Nähe zu einer befahrenen Straße bewegen, wodurch ihr Schutz vor einem möglichen Verkehrsunfall nicht 
sichergestellt ist. Die Tatsache, dass die Regel auf der einen Seite von der Kinderbetreuerin benannt wird, sie auf der anderen Seite jedoch von Seiten der Mitarbeiter*innen nicht eingehalten wird, könnte auf einen gewünschten Umgang mit Aspekten von Kinderschutz in der Institution hindeuten, der an einer Wirklichkeit scheitert, in der eine strukturell bedingte Knappheit an personellen und finanziellen Ressourcen vorherrscht (zur Betreuungssituation und personellen Ressourcenknappheit in der Unterkunft siehe auch den Beitrag von Scott \& Le).

Neben der beschriebenen Diskrepanz zwischen Regeln und Praxis weist das Datenmaterial auch auf mangelnde Regelungen und mangelnde Sensibilität für Risiken und Prävention von Gewalt gegen Kinder hin. Als Beispiel möchte ich im Folgenden eine Situation analysieren, die von den Forscherinnen in der kleineren Gemeinschaftsunterkunft beobachtet wurde. Es handelt sich um eine Situation zwischen einer technischen Hilfskraft der Unterkunft und dem Mädchen Elsa. Die Hilfskraft ist bei den Eltern des Mädchens, sowie bei anderen Eltern, die in der Unterkunft leben, beliebt. So bezeichnet ihn eine Mutter als hilfsbereiten und kinderfreundlichen Mann, den alle Kinder mögen würden, und erzählt weiter: [E]r lässt alles stehen und liegen und spielt mit den Kindern (Feldbesuch 7, GU 1, 28.7.2016). Auch eine Mitarbeiterin erwähnt in einem Interview: Er kann ganz gut mit kleinen Kindern (Interview, GU 1, 15.12.2016), und die Heimleitung erzählt den Forscherinnen: [D]er liebt alle Kinder, und hält kurz inne. Also nicht soo ... [...] Ich vertraue ihm (Feldbesuch 11, GU 1, 31.8.2016). Die Situation, die aus einer Kinderschutzperspektive analysiert werden soll, wird in dem Beobachtungsprotokoll folgendermaßen beschrieben: Die technische Hilfskraft fragt Elsa in Anwesenheit ihres Vaters, ob sie mit ihm in den Keller gehen möge - möglicherweise um sie ein wenig aufzuheitern, da sie an dem Tag offenbar bedrückt ist. Elsa geht mit dem Mann mit, und der Vater hat offenbar keine Einwände (vgl. Feldbesuch 6, GU 1, 21.7.2016). Dies deutet darauf hin, dass die technische Hilfskraft für den Vater eine Vertrauensperson ist. Die Aussage des Vaters, seine Tochter loves [Kinderbetreuerin] and [technische Hilfskraft] (ebd.), verstärkt diese Interpretation. Kurz darauf wird beschrieben, wie Elsa, gefolgt von der technischen Hilfskraft, in den Kinderbetreuungsraum rennt und mit Vehemenz versucht, den Mann nicht in den Raum eintreten zu lassen. Sie schreit laut, schließt das Kindersicherungstürgitter sowie die Tür und schiebt ihn letztlich mit den Händen zur Tür hinaus (vgl. ebd.). Elsas Motiv bleibt bei der vorliegenden Informationslage offen. Da wir nicht wissen, welche Interaktion sich in dem Keller (sofern sie tatsächlich in den Keller gegangen sind) zwischen Elsa und der technischen Hilfskraft ereignet hat, ist es nicht zielführend, an dieser Stelle nach Erklärungen für die oben beschriebene Reaktion des Mädchens auf die Hilfskraft zu suchen. Da es nach der mir vorliegenden Informationslage niemanden gibt, der die beiden begleitet oder beobachtet hat, kann weder Elsa durch die Anwesenheit oder Intervention anderer Personen vor möglichen 
Übergriffen geschützt werden noch ist die Hilfskraft vor einem möglicherweise aufkommenden unbegründeten Verdacht der Kindeswohlgefährdung geschützt. So ist es aus einer Kinderschutzperspektive äußerst bedenklich, dass die technische Hilfskraft alleine mit Elsa in den Keller geht bzw. dass die Strukturen der Einrichtungen dies zulassen. Betonen möchte ich, dass es mir nicht darum geht $\mathrm{zu}$ argumentieren, dass nichtpädagogisches Personal keine Beziehung zu Kindern aufbauen darf. Im Gegenteil, Beziehungen zum Personal der Unterkünfte können - darauf weist das Datenmaterial hin - für Kinder wertvolle Ressourcen darstellen. Vielmehr argumentiere ich, dass Momente, in denen sich Mitarbeiter*innen ungesehen von anderen Menschen alleine mit einem Kind aufhalten, Risiken für mögliche Übergriffe des Erwachsenen gegenüber dem Kind oder für das Aufkommen eines Verdachts für einen möglichen Übergriff bergen können. Dieses Beispiel macht deutlich, wie wichtig ein klar geregeltes Kinderschutzsystem in der Unterkunft ist, welches sowohl Elemente der Prävention von Gewalt gegen Kinder als auch ein Beschwerde- und Fallmanagement beinhaltet und welches insbesondere Kindern bekannt und zugänglich sowie wirkungsvoll ist. So ist es von großer Bedeutung, dass Kinder in der Unterkunft die Möglichkeit haben, sich bei möglichen Übergriffen beschweren zu können und Schutz bei Menschen zu suchen, denen sie vertrauen. Gleichzeitig ist es wichtig, dass (anwesende) erwachsene Personen mit der Frage nach einer adäquaten Reaktion auf die Situation wie der oben beschriebenen und nach einem angemessenen Umgang mit ihnen nicht überfordert sind. Aus der Beschreibung der geschilderten Situation geht beispielsweise nicht hervor, ob eine der anwesenden Personen Elsa in der geschilderten Situation gefragt hat, warum sie die Tür zuhalte und die Hilfskraft nicht in den Raum lasse. Die Herstellung des Kontakts zu dem Mädchen und das Eintreten in eine der Situation gerecht werdenden Kommunikation mit ihm sind für den Schutz des Kindes und nicht zuletzt für das Einschätzen der Situation von groBer Bedeutung. Dieses Beispiel zeigt auch, wie wichtig es ist, dass alle Mitarbeiter*innen in Schulungen zu Kinderrechten und Gewaltschutz einbezogen werden, auch wenn diese nicht zum sozialpädagogischen Personal gehören.

Nachdem die Forscherinnen die Hinweise und Bedenken im Rahmen einer Feedbackrunde zu ihrer Forschung an die Einrichtungsleitung und Mitglieder des Teams gespiegelt hatten, setzte sich das Team der Unterkunft laut der Heimleitung intensiv mit diesem Thema auseinander und die Heimleitung überarbeitete das Gewaltschutzkonzept sowie die Dienstanweisungen, wobei sie sich an einer Veröffentlichung zu Kinderschutz in Gemeinschaftsunterkünften orientierte. In den Dienstanweisungen stehe nun auch ganz klar, wer sich im Fall von Gewaltanwendung an wen zu wenden habe (vgl. Telefonnotiz, GU 1, 21.6.2017). Darüber hinaus nehme die Kinderbetreuerin nun regelmäßig an einer Runde zu Kinderschutz vom Bezirksamt teil, wobei es im Datenmaterial keine weiteren Hinweise zu dieser Runde gibt. Inwieweit sich die 
Kinderschutzpraxis in der Unterkunft tatsächlich verändert hat, können wir aufgrund des Austritts aus dem Feld jedoch nicht beurteilen.

Ein weiterer Aspekt, der mir in der Analyse der Daten aufgefallen ist, sind Unklarheiten in Rollen und Verantwortlichkeiten im Kinderschutz sowohl beim Unterkunftspersonal als auch bei zuständigen Stellen der Kinder- und Jugendhilfe. Als Beispiel hierfür wird eine Szene aus dem Datenmaterial der größeren Gemeinschaftsunterkunft herangezogen. In einem Interview mit der Kinderbetreuerin und der Heimleiterin dieser Unterkunft wird die Situation einer Familie angesprochen, die mit einer sogenannten Duldung in Deutschland lebt. Laut der Kinderbetreuerin wurde bei dem Sohn der Familie, Spiderman, eine Traumatisierung festgestellt und auch die Mutter beschreibt Anzeichen einer Traumatisierung ihres Kindes. Offenbar wünschen sich die Eltern Unterstützung für ihren Sohn, so habe die Familie laut der Kinderbetreuung auch aus diesem Grund an dieser Forschung teilgenommen. Die Heimleiterin berichtet in Bezug auf die Situation der Familie von einem Kontakt mit dem Jugendamt:

[Heimleiterin:] Na, es war jemand vom Jugendamt auch hier, ne? Hat das Gespräch mit uns als Einrichtung wie aber auch mit der Familie gesucht. Genau, und die haben gesagt, also, sie ${ }^{10}$ sollen jetzt mal gucken, wenn das Kind auffällig ist oder man das Gefühl hat, ne? Da bleibt also, da bleiben schlechte Erinnerungen oder dass sie dann, genau, den Kinder-, wie heißt das?

[Sarah] und [Mai]: (gleichzeitig) Kinder- und Jugendpsychiatrischer Dienst.

[Heimleiterin:] Genau, dass der, dass sie da vorstellig werden müssen, damit sie am Ende eine Therapie bewilligt kriegen. So, das war mein letzter Stand. Mehr weiß ich jetzt nicht. Ob sie das dann gemacht haben? (Interview bei dem Feldbesuch 10, GU 2, 20.5.2017)

Insgesamt bleibt offen, wie konkret das Angebot an die Familie in Bezug auf einen Therapieplatz für Spiderman bereits war und inwiefern der Familie die Chance auf einen Therapieplatz tatsächlich verständlich kommuniziert worden ist. Selbst die Heimleiterin stolpert über den Kinder- und Jugendpsychiatrischen Dienst, was darauf hinweist, dass sie in Bezug auf die Rolle und Zuständigkeit des Dienstes unsicher ist. Die Unklarheit der Heimleiterin über den aktuellen Stand der Familie in Bezug auf die unternommenen Schritte für die Bewilligung eines Therapieplatzes deutet darauf hin, dass es an einer klaren Kommunikationsstruktur der unterschiedlichen Akteur*innen für die Begleitung und Unterstützung der Familie mangelt und die Verantwortlichkeiten offenbar nicht klar geregelt sind. Wie das folgende Zitat der Kinderbetreuerin

10 Es ist unklar, ob hier das zuständige Personal im Allgemeinen gemeint ist oder direkt die Heimleiterin angesprochen wird. Wäre letzeres der Fall, müsste das „sie“ in „Sie“ umformuliert werden. 
zeigt, spielen auch Rollenkonflikte eine Rolle für Unsicherheiten in der Begleitung der Familie:

Apropos Rollenkonflikt, das sind Sachen, die ich da beobachtet habe, als ich dann zum Essen eingeladen wurde. Und dann dachte ich, ich will auch nicht, dass die das Gefühl haben, wenn sie mich zum Essen einladen, dass ich jetzt alles scanne und dann einen Bericht schreibe. [...] In welcher Rolle stehe ich jetzt? (ebd.)

So sieht sich die Kinderbetreuerin in einem Rollenkonflikt, inwiefern sie sich als Kinderbetreuerin und gleichzeitig Freundin der Familie beispielsweise in Erziehungsfragen einmischen darf. Insgesamt weist das Interview an verschiedenen Stellen darauf hin, dass die Mitarbeiter*innen trotz ihres Engagements und ihrer Bemühungen mit einer erfolgreichen Vermittlung der Familie an Unterstützungsangebote aus der Kinder- und Jugendhilfe unsicher sind. Dies könnte auf eine auf struktureller Ebene vorliegende Unklarheit der Zuständigkeiten in Bezug auf die Situation von Kindern in Unterkünften für geflüchtete Menschen zurückzuführen sein. Wie viel schwerer muss es angesichts der Unsicherheiten der Sozialarbeiter*innen und der Heimleitung also für die Familien sein, die sich in dem System nicht gut auskennen, die Sprache nicht gut sprechen, nur eingeschränkt Internetzugang haben und darüber hinaus mit weiteren hohen Belastungen konfrontiert sind, Unterstützungsangebote, die ihnen zustehen würden, wahrzunehmen? Angesichts einer fehlenden Ansprechperson informieren die Forscherinnen letztlich die Familie über die Situation und setzen sich über zwei Beratungs- und Therapiestellen dafür ein, Spiderman einen Therapieplatz zu vermitteln. Doch leider erhalten sie die Antwort, dass die Stellen überlastet seien und dem Jungen, vor allem vor dem Hintergrund, dass er mit einem unsicheren Aufenthaltsstatus in Deutschland lebt, keinen Therapieplatz anbieten könnten. Zusammenfassend lässt sich sagen, dass dieses Beispiel deutlich macht, dass das Kinder- und Jugendhilfesystem und die Zusammenarbeit aller zuständigen Akteur*innen zur Unterstützung der Familie nicht ausreichend funktionieren, die Eltern in ihrer Erziehungsverantwortung nicht ausreichend gestärkt werden und das Kind sein Recht auf Entwicklung (Artikel 6 UNKRK) nicht in vollem Umfang wahrnehmen kann.

Betrachten wir die oben aufgeführten Beispiele des Umgangs des Unterkunftspersonals mit relevanten Fragen und Aufgaben des Kinderschutzes kann die Schlussfolgerung gezogen werden, dass die strukturellen Bedingungen in den Unterkünften - die unter anderem geprägt sind vom Mangel an qualifiziertem und sensibilisiertem Personal, von prekären Arbeitsbedingungen und von finanzieller und materieller Ressourcenknappheit - die Qualität der Arbeit negativ beeinflussen und zu einer Überforderung der Mitarbeiter*innen in der Wahrnehmung kinderschutzrelevanter Aufgaben beitragen. Deutlich betonen möchte ich an dieser Stelle den Zusammenhang zwischen den überall festgestellten prekären Arbeitsbedingungen des Unterkunftspersonals, die nach dem 
hier zugrunde liegenden Gewaltverständnis eine Form struktureller Gewalt darstellen, und der von mir interpretierten Überforderung des Personals in der Wahrnehmung kinderschutzrelevanter Aufgaben. So wird klar, wie sehr der Schutz von Kindern vor Gewalt mit den strukturellen Gegebenheiten der Unterkünfte verwoben ist.

Neben den dargestellten Herausforderungen weisen alle drei Unterkünfte bei einer Analyse des Datenmaterials auch wertvolle Ressourcen und Potentiale im Bereich Kinderschutz auf. Hervorzuheben sind insbesondere die vielen engagierten Mitarbeiter*innen, die unter hohen Belastungen und schwierigen Arbeitsbedingungen Familien, die in den Unterkünften leben, Ansprechpartner*innen für die Familien sind, sie begleiten und ihnen Unterstützungsangebote machen. So wird aus dem Datenmaterial deutlich, dass Sozialarbeiter*innen bei der Vermittlung von Kitaplätzen, bei der Vermittlung von Ärzt*innen mit einer relevanten Sprachkompetenz für die Bewohner*innen, beim Verstehen und Ausfüllen von Behördenschreiben, bei der Vermittlung von Dolmetscher*innen und Unterstützungsprogrammen wie Netzwerkkoordinator*innen oder bei der Vermittlung juristischer Hilfe helfen. Im Datenmaterial der kleineren Gemeinschaftsunterkunft findet sich beispielsweise die Beschreibung einer Situation, in der ein Sozialarbeiter der Unterkunft eine Familie, die eine Abschiebebescheid für den Vater erhalten hat, beim Erheben eines Widerspruchs unterstützt und ihnen mögliche Wege aufzeigt, um eine Abschiebung zu verhindern. Zwar gibt es keine Hinweise im Datenmaterial darüber, wie die Familie die Rolle des Sozialarbeiters wahrnimmt. Eine Einschätzung der Forscherin ist jedoch zu finden:

Dennoch hatte ich das Gefühl, dass sich der Sozialarbeiter um das Wohl der Familie sorgte, sich auskannte und sofort Bescheid wusste, was mich beruhigte. Er würde sich etwas einfallen lassen, wenn das mit dem Eilantrag nicht klappt. Seine gefasste, zuversichtliche und ruhige Ausstrahlung hatte meinen innerlichen Aufruhr beruhigt (Feldbesuch 6, GU 1, 21.7.2016).

Tatsächlich erhält die Familie später die Möglichkeit, in Deutschland einen Asylantrag zu stellen (für eine ausführliche Darstellung der Situation der Familie siehe den Beitrag von Fichtner \& Trần ). Wie dieses Beispiel zeigt, kann die Unterstützung und Stärkung geflüchteter Familien dazu beitragen, dass Kinder vor gewaltvollen Praxen wie der Abschiebung geschützt werden können. Betont werden soll an dieser Stelle jedoch, dass Sozialarbeiter*innen hier nur eine unterstützende Rolle einnehmen können. Die konsequente Verhinderung von Menschenrechtsverletzungen von Kindern durch Abschiebungen müsste durch eine Veränderung auf struktureller Ebene stattfinden. Ferner kann die Unterstützung von Familien in der Bewältigung von Herausforderungen und Hürden, denen sie in Deutschland begegnen, dazu beitragen, dass die Eltern entlastet werden und dadurch ihre Fähigkeit, ihre Kinder vor Gewalt zu schützen, gestärkt wird (vgl. BVKJ 2016: 24, 86). Darüber hinaus bieten die 
Unterkünfte eine Kinderbetreuung an, in der Kinder Raum zum Spielen haben und Eltern in der Betreuung ihrer Kinder entlastet werden. Spidermans Mutter, die mit ihrer Familie in der größeren Gemeinschaftsunterkunft lebt, erzählt beispielsweise, es gebe sehr viele Freizeitangebote für die Kinder. , Es gibt vieles, was gut ist", sagte Spidermans Mutter , ,und sie lernen was Nützliches dabei, und das ist auch gut für die Schule " (Feldbesuch 7, GU 2, 2.2.2017). Der Umgang von Mitarbeiter*innen der Unterkünfte mit den Kindern wird von den Forscherinnen in den Beobachtungsprotokollen zwar nicht durchgehend, jedoch an vielen Stellen positiv hervorgehoben und als respektvoll, geduldig und pädagogisch wertvoll wahrgenommen. Das Aufzählen oder Malen bestimmter Kinderbetreuer*innen oder Sozialarbeiter*innen neben anderen Bezugspersonen der Kinder wie den Eltern, Geschwistern, Freund*innen, Erzieher*innen aus der Kita sowie den Forscherinnen weist darauf hin, dass einige Mitarbeiter*innen für Kinder, die in den Unterkünften leben, offenbar wichtige Bezugspersonen darstellen. Hervorheben möchte ich außerdem Kooperationen mit relevanten Stellen im Bereich Kinderschutz unter anderem mit dem Jugendamt, mit Kitas, mit Schulen, mit einem Netzwerkkoordinator*innenprogramm sowie mit einer Projektinitiative zum Schutz von Frauen und Kindern in Unterkünften für geflüchtete Menschen von UNICEF und dem Bundesministerium für Familie, Senioren, Frauen und Jugend (BMFSFJ). All diese Aspekte möchte ich - ohne in der Analyse der Ressourcen der Unterkünfte Anspruch auf Vollständigkeit erheben zu wollen - positiv hervorheben.

Aus der Analyse des mir vorliegenden Datenmaterials kann zusammenfassend gesagt werden, dass die ,general principles“ der UNKRK für die Umsetzung des Menschenrechts von jungen Kindern auf Schutz vor Gewalt im Umgang der Mitarbeitenden bzw. Einrichtungen und darüber hinaus im Kinderund Jugendhilfesystem mit dem Thema nicht ausreichend berücksichtigt werden. Insbesondere inwieweit Kinder in Entscheidungen oder die Planung und Umsetzung von Aktivitäten und Maßnahmen im Bereich Kinderschutz einbezogen werden und somit ihr Recht auf Partizipation im Schutz vor Gewalt wahrnehmen können, ist kritisch zu hinterfragen. Tatsächlich gibt es im Datenmaterial kaum Hinweise dafür. Mindestens zwei Deutungen lässt dieser Befund zu: Die teilnehmende Beobachtung und Datenprotokollierung der Forscherinnen hat dieser Thematik nicht den entsprechenden Stellenwert beigemessen bzw. lag ihr Fokus auf anderen Bereichen. Diese Deutung ist jedoch vor dem Hintergrund einer Forschung, die angetreten ist, die Belange und Interessen von Kindern in den Unterkünften aus Ausgangspunkt ihrer Erhebung zu nehmen, wenig überzeugend. In Anbetracht der Tatsache, dass in Unterkünften nach $\S 44$ Abs. 3 AsylG eine Betriebserlaubnis nach $\S 45$ Abs. 2 Satz 2 SGB VIII die Notwendigkeit von ,geeignete[n] Verfahren der Beteiligung sowie der Möglichkeit der Beschwerde in persönlichen Angelegenheiten“" geregelt wird, nicht erforderlich ist, ist davon auszugehen, dass es keine bzw. kaum etablierte Beteiligungsformen für und mit Kindern in den Unterkünften gibt 
(siehe hierzu auch die Mindeststandards zum Schutz von geflüchteten Menschen in Flüchtlingsunterkünften von UNICEF und dem BMFSFJ: 2017). Welche Ressource und welches Potential jedoch die Partizipation von Kindern für ihren Schutz vor Gewalt darstellen kann, diskutiere ich im folgenden Kapitel.

\section{Kinder als kompetente Akteure für den Schutz vor Gewalt}

Kinder haben einen menschenrechtlichen Anspruch darauf, aktiv an ihrem Schutz vor Gewalt mitzuwirken. Tatsächlich finden sich im Datenmaterial Belege dafür, dass junge Kinder in den Unterkünften über Ressourcen für ihren Schutz vor Gewalt verfügen und sie nutzen oder einsetzen. Im Folgenden möchte ich nun exemplarisch anhand von zwei Situationen aufzeigen, wie sich Kinder vor Gewalt schützen bzw. sich gegen Gewalt wehren.

\section{1 „MANNO STOPP!“}

In einem Beobachtungsprotokoll aus der kleineren Gemeinschaftsunterkunft wird eine Situation beschrieben, die sich auf einem Spielplatz im Hof der Einrichtung abspielt, auf den die Forscherin die Mutter Kizhan und ihre Tochter Elsa begleitet hat. Es wird beschrieben, dass ein Mann, welcher die Rolle einer technischen Hilfskraft in der Unterkunft einnimmt, zielstrebig auf Elsa, die sich gerade auf einem Wipptier befindet, zugeht, bis er ganz nah neben ihr war und in deutlichem, lautem und in tiefem Ton in ihr Ohr sprach: ,Zieh deine Schuhe an "-was wie ein straffer Befehl klang (Feldbesuch 4, GU 1, 5.7. 2016). Aus einer adultismuskritischen Perspektive kann die Situation so interpretiert werden, dass der Mann seine Macht als Erwachsener gegenüber dem Kind demonstriert. Er gibt Elsa keine Begründung für seine Aufforderung, so dass sie seine Aufforderung möglicherweise nicht nachvollziehen kann. Elsas Sichtweise darüber, ob sie Schuhe tragen möchte oder nicht, wird von ihm weder abgefragt noch berücksichtigt und auch die Mutter des Kindes wird von ihm diesbezüglich weder einbezogen noch beachtet. Elsa zieht ihre Schuhe jedoch nicht an und leistet somit der Aufforderung des Mannes nicht Folge. Die körperliche Nähe des Mannes scheint Elsa unangenehm zu sein: Elsa rückte sofort zur Seite, soweit es ihr oben auf dem kleinen Wippplatz möglich war (ebd.). Durch ihr unmittelbares Wegrücken signalisiert sie, wo ihre Grenze der körperlichen Nähe zu der technischen Hilfskraft ist, und verschafft sich wieder 
eine körperliche Distanz zu dem Mann. Doch der Mann wich nicht von ihr ab, versuchte es aber mit etwas anderem: „Pass auf! “ (ebd.) Wieder kommuniziert er mit ihr in Form einer Aufforderung, die auch als Befehl oder Drohung gelesen werden kann. Aus dem Datenmaterial wird nicht deutlich, worauf sich seine Ansage beziehen könnte. Aus einer adultismuskritischen Perspektive kann interpretiert werden, dass er diese Ansprache wählt, da er sich als Erwachsener in einer adultistisch geprägten Gesellschaft berechtigt dazu fühlt, ein Kind auf diese Art und Weise zu etwas aufzufordern. Auch die beobachtende Forscherin interpretiert, dass er das Bedürfnis hat, mit dem Kind in Interaktion zu treten (ebd.). Doch Elsa reagierte nicht wirklich auf seine Worte (ebd.). Dieses Nichtreagieren könnte als ein erneutes Signal gedeutet werden, dass sie in Ruhe gelassen werden möchte. Nachdem Elsa dem Mann auch nach seiner zweiten Intervention keine Aufmerksamkeit geschenkt hatte, versuchte [der Mann] es dann mit Anwippen und stellte sich auf die Wippe in der Mitte, um das Wipperlebnis von Elsa ,intensiver" zu machen und sprang leicht auf und $a b$ (ebd.). Damit greift er in das Spiel des Mädchens ein, ohne sie vorher um Erlaubnis gebeten zu haben. Kizhan scheint um die Sicherheit ihrer Tochter besorgt zu sein, da sie sich als Reaktion darauf in die unmittelbare Nähe ihrer Tochter (ebd.) begibt und diese auffordert, sich an der Metallstange festzuhalten. Möglicherweise möchte sie ihrer Tochter auch Halt und Sicherheit gegenüber der Intervention der technischen Hilfskraft geben. Indirekt könnte ihre Reaktion auch als ein Signal an den Mann interpretiert werden, mit dem Anwippen des Mädchens aufzuhören. Als der Mann dann schließlich von der Wippe absteigt, geht er wieder auf Elsa zu.

Er war wieder sehr nah bei ihr, berührte sie, glaube ich, auch an ihrer Hand. Ihre Reaktion fand ich bemerkenswert, sie wollte alleine wippen und streckte dem Mann neben ihr ihre Zunge heraus und sagte: „Bäh!“, er wich nicht von ihrer Seite und wippte sie zusätzlich von der Seite an, so dass sie vorsichtiger sein musste, weil das Wippen dadurch unregelmäßiger wurde. Sie sagte daraufhin: ,,Nein, nein, nein! “, signalisierte ihm, dass er aufhören sollte, was er nicht tat. Dann sagte sie: ,So ... so ... stopp!" und wiederholte das einige Male, bis ein lauter, explizier, fast genervter Ton kam: „MANNO STOPP!“ (ebd.)

Erneut wehrt sich Elsa gegen die Grenzüberschreitungen des Mannes, die ich als übergriffig und gewaltvoll interpretiere, und macht mit klaren Worten wie „nein“ und „stopp“ darauf aufmerksam, dass sie beim Wippen nicht gestört werden möchte. Da er nicht aufhört, wird sie irgendwann laut und ruft: „MANNO STOPP!“”, um sich Gehör zu verschaffen. Sie schützt sich dadurch vor dem Verhalten des Mannes, indem sie Agency zeigt, worunter James \& James (2008: 9) ,the capacity of individuals to act independently“ und ,children's ability [...] to have some control over the direction their own lives take" verstehen. Da der Mann nicht von Elsas Seite weicht, zieht sie sich von ihm zurück und geht auf die Schaukel. Doch der Mann folgt ihr erneut und bewirft sie zwei Mal mit einem Ball. Erst als Elsas Mutter interveniert, indem sie mit 
ihrer Tochter in der Muttersprache spricht, den Ball in die Hand nimmt und ihn Elsa zuspielt, geht der Mann. Durch ihr Handeln unterstützt sie ihre Tochter in ihrem Anliegen, von dem Mann in Ruhe gelassen zu werden. Offenbar bedarf es trotz der vielen Signale des Mädchens dieser Intervention der Mutter, damit der Mann von dem Kind ablässt und geht. Insgesamt wird in dieser Szene immer wieder das Verhältnis von Nähe und Distanz zwischen Elsa und dem Mann verhandelt. Hierbei wird deutlich, wie kompetent Elsa ihre eigenen Grenzen erkennt, diese dem Mann kommuniziert und wie vehement sie sich gegen ein Überschreiten dieser Grenzen wehrt. Diese Fähigkeit gilt als ein wichtiger Faktor von Resilienz von Kindern und wird in der Kinderschutzforschung als ein Schutzfaktor vor Gewalt angesehen (vgl. Fröhlich-Gildhoff \& Rönnau-Böse 2009: 52).

Als kontextrelevanter Faktor zur Stärkung der Handlungsfähigkeit kann hier die Anwesenheit der Mutter gezählt werden, unter Umständen hat auch die Präsenz der Forscherin dazu beigetragen, dass Elsa sich gegen die Interventionen des Mannes wehren konnte. Das Datenmaterial weist zudem darauf hin, dass Elsa eine starke Bindung zu ihren beiden Elternteilen hat und diese sehr präsent sind und einen liebevollen und umsorgenden Umgang mit ihrer Tochter haben. Brooks \& Goldstein (2017) heben Bindungsfähigkeit als wichtige Ressource für Handlungsfähigkeit und die Entwicklung von Resilienz hervor. Auch andere Bezugspersonen, wie die Kinderbetreuerin der Einrichtung und die Forscherinnen scheinen für Elsa wichtig zu sein, da sie diese zusammen mit sich und ihren Eltern aufzählt, während die Forscherin die Namen für Elsa sichtbar in ein Notizbuch schreibt (vgl. Feldbesuch 6, GU 1, 21.7.2016). Zudem erzählt die Mutter, dass Elsa gerne in die Kita gehe, und zählt mithilfe des Mädchens die vielen Freund*innen auf, die sie in der Kita habe (vgl. Feldbesuch 4, GU 1, 5.7.2016). Auch die Heimleiterin erzählt, dass Elsa in der Kita sehr beliebt sei, und schätzt sie als ein Kind mit einer hohe[n] soziale[n] Kompetenz (Feldbesuch 11, GU 1, 31.8.2016) ein. Die Forscherinnen beschreiben Elsa ebenfalls als kontaktfreudig und überwiegend als fröhlich und aufgeweckt (vgl. Feldbesuch 4, GU 1, 5.7.2016 und Feldbesuch 6, GU 1, 21.7.2016). Nicht zuletzt kann auch Elsas Verhältnis zu der technischen Hilfskraft eine Rolle dabei spielen, dass sie ihm in der Art und Weise gegenübertritt, wie es oben beschrieben wird. An verschiedenen Stellen im Datenmaterial benennt Elsa die Hilfskraft bei ihrem Namen, bei einer Raumbegehung mit den Forscherinnen klebt sie einen lachenden Smiley an seine Tür und Elsas Vater betont mehrere Male, dass Elsa ihn möge. Diese Aspekte deuten darauf hin, dass die technische Hilfskraft für Elsa eine Bezugsperson ist. All diese aufgeführten Faktoren können eine Rolle dabei spielen, dass Elsa in der Interaktion mit der Hilfskraft Agency zeigt (siehe den Beitrag von Fichtner \& Trần). 


\subsection{Die „Kinderrevolution“}

Eine äußerst bemerkenswerte Situation, die sich in der größeren Gemeinschaftsunterkunft ereignet hat, macht deutlich, wie sich Kinder gegen Gewalt, die sie erfahren haben, organisieren und wehren können. Die Forscherinnen erfahren von der Situation zunächst von einer Kinderbetreuerin, die folgendes berichtet:

Letzte Woche hat es eine regelrechte Revolution der Kinder gegeben. Sie haben sich bei der Leitung über einen Kinderbetreuer beschwert. Sie haben in Sprechchören gerufen: „[Kinderbetreuer] muss weg, [Kinderbetreuer] muss weg ...! ", weil er zu streng ist. Drei Neunjährige waren die Anführer und zwanzig andere haben sich angeschlossen, auch die Kleinen (Feldbesuch 9, GU 2, 14.2.2017).

Die Heimleiterin schildert in einem Interview, wie eine Gruppe von Kindern unterschiedlichen Alters eines Abends an ihrer Bürotür klopfte und sich bei ihr über den Kinderbetreuer beschwerte.

Ich war natürlich überrascht, hab die aber auch alle ernst genommen. Wir haben uns dann an den runden Tisch gesetzt bei mir im Besprechungszimmer. Und das haben sie auch am Anfang sehr, sehr diszipliniert gemacht, indem sie wirklich auch toll vorgetragen haben, um was es geht und was das Anliegen ist; haben's auch begründet (Interview bei dem Feldbesuch 10, GU 2, 20.5.2017).

Die Kinder wussten offenbar, dass sie sich mit ihrer Beschwerde an die Vorgesetzte des betroffenen Kinderbetreuers wenden mussten. Indem sie das Gespräch mit der Heimleitung gesucht haben, verschaffen sie sich Gehör, um für ihr Anliegen einzutreten. So berichten die Kinder selbst: Wir, die Kinder, haben Chef gesagt! (Feldbesuch 9, GU 2, 14.2.2017) Die Tatsache, dass sie sich an die Heimleitung wenden, deutet auf klare Strukturen und Hierarchien in der Einrichtung hin. Zudem wissen die Kinder offenbar um die strukturelle Abhängigkeit von der Heimleitung, die sie in ihrer Lebenssituation in der Einrichtung haben. Ferner könnte die Kontaktaufnahme auch auf ein positives Verhältnis zwischen ihnen deuten, wobei das Datenmaterial hierzu keine näheren Informationen gibt. Die Heimleiterin berichtet, dass sie sich zunächst einmal angehört habe, was die Kinder zu sagen hatten. Als der betroffene Kinderbetreuer dann zufällig ihr Büro betrat, habe sie ihn mit an den runden Tisch gebeten, so dass sie beide Seiten hören konnte. Trotz einer recht ruhigen Atmosphäre konnten sie in dem Gespräch jedoch nicht zu einer Lösung oder gemeinsamen Vereinbarung bezüglich des weiteren Umgangs miteinander kommen. Dies habe ihrer Meinung nach zum einen daran gelegen, dass der Erwachsene sich nur gerechtfertigt habe, zum anderen daran, dass die Kinder erschöpft und der Bogen überspannt war (Interview bei dem Feldbesuch 10, GU 2, 20.5.2017). Dies führt sie jedoch nicht näher aus. 
Als Reaktion auf die Initiative der Kinder sucht die Heimleiterin zunächst das Gespräch mit dem betroffenen Kinderbetreuer und beruft anschließend eine Teambesprechung ein, um zu gucken, wie gehen wir jetzt mit der Situation um - das einfach zu negieren, geht natürlich auch nicht (ebd.). Im Team wurde beschlossen, das Thema in Gesprächen mit Kinderbetreuer*innen und Kleingruppen von Kindern aufzugreifen, um den Konflikt zu bearbeiten und eine Lösung zu finden. Eine Kinderbetreuerin schildert den Forscherinnen, dass die Gespräche sehr, sehr emotional (ebd.) verliefen und nicht dazu geführt hätten, dass sich die Beziehung zwischen den Kindern und dem Kinderbetreuer verbessert habe. Der Kinderbetreuer sei in den Augen der Kinder unglaubwürdig und das Vertrauensverhältnis gestört gewesen. Letztendlich beendete ein Kind, das in der Gruppe der Kinder als Boss (ebd.) angesehen wurde, das Gespräch. Die Kinderbetreuerin berichtet:

Und da meinte er [das Kind]: „, Okay, das Gespräch ist hier zu Ende. Wir gehen jetzt. “[...] Sagte: ,,Du sprich jetzt nicht mit ihm. “ [...] Und [...] nur ein Kind ist drinnen geblieben (ebd.).

An dieser Stelle gibt es Hinweise darauf, wie die Mitarbeiterin die Organisation der Kinder wahrnimmt. Sie berichtet von einem Boss unter den Kindern, der die Entscheidung treffe, das Gespräch zu beenden und zu gehen. Die Reaktion der Kinder zeigt, dass sie sich auf eine Versöhnung mit dem Betreuer offenbar nicht einlassen wollten. Sie forderten, dass er die Einrichtung verlässt.

Um die Perspektive der Kinder auf die Situation einzufangen, suchten die Forscherinnen, wie die folgende Sequenz aus einem Beobachtungsprotokoll zeigt, das Gespräch mit sechs Jungen:

Als ich die Kinder zur ,Kinderrevolution “ befragte, hatten alle Jungen viel zu sagen und sprachen durcheinander. Viele Jungen machten deutlich, wie der Kinderbetreuer die Kinder am Kragen gepackt hatte, um sie aus dem Kinderland zu werfen. Ein Junge sagte: „Er hat zu drei Kindern gesagt: ,Ihr schlaft auf der Straße!' "Ein weiterer Junge erklärte, dass die drei Jungen nicht den Raum sauber gemacht hätten. [...] Weiterhin wurde gesagt, dass der Kinderbetreuer die Kinder auch mehrmals geschubst habe. [...] Der Junge im Rollstuhl meinte, dass er auch mit seinem Rolli geschubst worden sei, und sagte: „Er hat gesagt, mein Papa ist ein Schwein. “ [...] Die Jungen meinten über den Kinderbetreuer, dass er nicht gut sei, und zeigten das auch mit einem Daumen nach unten (Feldbesuch 9, GU 2, 14.2.2017).

An dem Ausschnitt des Protokolls wird deutlich, dass das Bedürfnis der Kinder, über ihr Anliegen zu sprechen, sehr hoch ist. Sie erzählen den beiden Forscherinnen von dem Verhalten des Kinderbetreuers ihnen gegenüber, welches sie offenbar als gewaltvoll und unfair empfinden. Zwar liefert das Datenmaterial keinen Aufschluss darüber, wie sich die Situationen zwischen dem Kinderbetreuer und den Kindern tatsächlich zugetragen haben und wie sich der Mitarbeiter tatsächlich verhalten hat. Nichtsdestotrotz hat dieser in seiner Rolle 
als Kinderbetreuer und als Erwachsener zunächst einmal eine machtvolle Position inne. In der Perspektive der Kinder hat er diese durch ein Verhalten, das von mir als adultistisch gedeutet wird, anscheinend missbraucht. Der weitere Verlauf des Gesprächs zeigt, dass das Thema unter den Kindern eine hohe Emotionalität auslöst. Auch im Gespräch mit den Forscherinnen fangen die Jungen [...] an, wieder zu rufen: „[Kinderbetreuer] muss raus. Raus, raus, raus! (ebd.) Sie haben offenbar das Bedürfnis, auf die Missstände, die sie sehen, laut und deutlich hinzuweisen, und fragen die Forscherinnen sogar, ob diese das nächste Mal Transparente mitbringen könnten, um für ihre Forderung zu demonstrieren.

Die Selbstorganisation und den Aktivismus der Kinder halte ich aus folgenden Überlegungen für äußerst bemerkenswert. Ihr Handeln ist Ausdruck von Agency, wobei das weiter oben eingeführte Konzept von Agency hier dahingehend ausgeweitet werden kann, dass neben der Einflussnahme auf das eigene Leben bis zu einem gewissen Grad auch die Fähigkeit der Kinder zum Tragen kommt ,to play some part in the changes that take place in society more widely" (James \& James 2008: 9). Ihr Handeln kann auch als ein Akt von Empowerment bezeichnet werden, was laut Berger \& Neuhaus (zitiert nach Herriger 2006: 14) zum Ziel hat, ,die Macht etwas gerechter zu verteilen - und das dort, wo es wichtig ist, nämlich im Hinblick auf die Selbstbestimmung und die Kontrolle der Menschen über das eigene Leben". So betonen auch die Heimleiterin und Kinderbetreuerin ihre Wahrnehmung, dass die Kinder durch ihr geschlossenes und entschiedenes Handeln Macht erlangt hätten.

[Heimleiterin:] [...] die Kinder, das kann ich schon einschätzen, haben am Ende so eine Eigendynamik, weil sie gemerkt haben, sie haben so eine Macht, ja? [...] Und ich glaub, [...] da waren auch tatsächlich, [...] also ich will nicht das Wort verfeindet nehmen, aber an sich rebellierende oder miteinander kämpfende oder sich abgrenzende Kindergruppen waren plötzlich eins, ja? Also ... [...] über Nationen und Altersgruppen und Geschlechter, sie waren sich, da waren sich alle einig (Interview bei dem Feldbesuch 10, GU 2, 20.5.2017).

So erweist sich das vereinte Handeln der Kinder und das Auftreten als Verhandlungspartner*innen für einen respektvollen und gewaltfreien Umgang der Erwachsenen mit ihnen als ein zentraler Bestandteil für ihren Schutz vor Gewalt. Hier haben sich die Kinder ihr Recht auf Partizipation bei ihnen betreffenden Anliegen gemeinsam eingefordert und erkämpft. Nachdem der Versuch einer Einigung zwischen Kindern und dem betroffenen Mitarbeiter gescheitert ist, [Heimleiterin:] haben wir uns jetzt auch getrennt voneinander. [...] Das war sicher ein, ein nicht ungravierender Anlass (ebd.).

Im Datenmaterial finden sich nur wenige und vage Hinweise dafür, welche Faktoren die Handlungsfähigkeit der Kinder positiv beeinflusst haben könnten. Diese Einschränkung ist der Tatsache geschuldet, dass die „Kinderrevolution“ vorrangig auf das Handeln älterer Kinder zurückgeht. Doch die Beteiligung 
der jüngeren Kinder unter sechs Jahren soll in seiner Bedeutung, auch für die älteren Kinder, nicht unterschlagen werden. Ein Aspekt für die Handlungsfähigkeit der Kinder könnte sein, dass die Unterkunft über einen im Verhältnis $\mathrm{zu}$ anderen Unterkünften recht geräumigen Kinderbetreuungsbereich verfügt. Dieser Bereich, von den Mitarbeiter*innen als Kinderland (Feldbesuch 1, GU 2, 9.12.2016) bezeichnet, ist gemäß Beobachtungen der Forscherinnen immer gut besucht und gibt Kindern unterschiedlicher Altersklassen die Möglichkeit, Beziehungen miteinander zu knüpfen. Darüber hinaus halten sich die Kinder außerdem auch in anderen Räumlichkeiten, wie den Fluren, auf. Positiv hervorzuheben ist zudem, dass die Heimleiterin und das Team das Anliegen der Kinder offenbar ernst nehmen und auf sie eingehen, was auf eine grundsätzlich positive Haltung hinweist. Auch die Forscherinnen bezeichnen insbesondere den Umgang einer Kinderbetreuerin mit den Kindern als respektvoll und geduldig (vgl. Feldbesuch 9, GU 2, 14.2.2017). Darüber hinaus protokolliert die Forscherin über den Kinderbetreuungsbereich: Ich entdeckte ein Plakat über der Garderobe, auf dem Sprüche standen wie „, Nein ist nein “, „Ich bestimme mit! " oder auch ,, Trau dich " in bunten Farben (Feldbesuch 6, GU 2, 26.1.2016). Auch wenn dies nichts darüber aussagt, wie die Themen, die hier berührt werden, tatsächlich mit den Kindern behandelt werden, weist dieses Plakat auf eine Sensibilität von Mitarbeiter*innen der Kinderbetreuung für die Mitbestimmung von Kindern sowie für ihre Fähigkeit, die eigenen Grenzen zu erkennen und zu kommunizieren, hin. Auch in den Berichten der teilnehmenden Beobachtung der Forscherinnen finden sich Momente, in denen Kinder in der Kinderbetreuung mitbestimmen können, beispielsweise in der Auswahl der Aktivitäten der Kinder im freien Spiel oder in der Auswahl von Liedern im Abschlusskreis (vgl. Feldbesuch 4, GU 2, 5.1.2017, Feldbesuch 6, GU 2, 26.1.2017, Feldbesuch 9, GU 2, 14.2.1017 und Feldbesuch 10, GU 2, 20.5.2017). Nicht zuletzt möchte ich hervorheben, dass sich die Einrichtung an unser Forschungsteam gewendet hat, um Interesse an einer Teilnahme an der Studie zu bekunden. Sie verspreche sich daraus Erkenntnisse für eine Verbesserung ihrer Arbeit. Dies zeugt von einer engagierten, offenen, lernenden und ressourcenorientierten institutionellen Kultur, was ebenfalls möglicherweise eine Wirkung auf die Handlungsfähigkeit der Kinder hat.

Aus meiner Perspektive ist die „Kinderrevolution“ ein Beispiel dafür, wie Kinder ihr Recht auf Partizipation und ihr Recht auf einen gewaltfreien Umgang mit ihnen einfordern und in ihrer Forderung von den betreffenden Entscheidungsträgern ernst genommen werden. Zudem weist das Anliegen der Kinder, gehört zu werden, sich zu beschweren und eine Veränderung einzufordern, auch darauf hin, wie relevant es ist, Beschwerdestellen und Partizipationsmöglichkeiten für Kinder, die in Unterkünften für geflüchtete Menschen leben, einzurichten. Dieses Beispiel der „Kinderrevolution“ kann dazu anregen, auch auf anderen Ebenen auf ein Umdenken und Handeln aller relevanten 
Akteur*innen einzuwirken, damit das Recht von Kindern auf Schutz vor Gewalt anerkannt und umgesetzt wird.

\section{$6 \quad$ Fazit}

In meinen Ausführungen anhand empirischen Datenmaterials habe ich dargelegt, dass es verschiedene Dimensionen der Gewalt gibt, mit denen Kinder, die in Unterkünften für geflüchtete Menschen in Berlin leben, konfrontiert sind. In diesem Beitrag konnte differenziert aufgezeigt werden, wie verschiedene Formen von Gewalt ineinandergreifen und sich wechselseitig beeinflussen, wenn nicht gar bedingen.

Hervorheben möchte ich insbesondere Formen struktureller Gewalt, der Kinder und Familien in den Unterkunftskontexten ausgesetzt sind. Diese sind in den Strukturen der Unterkünfte und im gesamten Asylsystem über geflüchtete Menschen verhaftet. Daneben weist das Datenmaterial auf Formen personaler Gewalt hin, darunter innerfamiliärer Gewalt, Gewalt zwischen Kindern und Gewalt im Umgang von Mitarbeiter*innen der Unterkünfte gegenüber Kindern.

Die Analyse des Umgangs der Mitarbeiter*innen mit dem Bereich Kinderschutz ergibt, dass in den Einrichtungen sowohl Ressourcen und Potentiale als auch Schwierigkeiten und Herausforderungen existieren. Wertvolle Ressourcen stellen vor allem die vielen engagierten Mitarbeiter*innen dar, die unter hohen Belastungen und prekären Arbeitsbedingungen Familien unterstützen, eine Kinderbetreuung anbieten und für viele Kinder wichtige Bezugspersonen darstellen. Klare Strukturen und Ansprechpersonen für Kinder sowie das Ernstnehmen und Eingehen auf Anliegen und Forderungen, die Kinder äußern, stellen ebenfalls wichtige Ressourcen und Handlungsweisen seitens der Einrichtung und des Personals dar. Doch unsere Forschung weist auch auf vielfältige Schwierigkeiten und Herausforderungen in der Kinderschutzpraxis der Unterkünfte hin. So zeigt sich im Datenmaterial eine Kluft zwischen aufgestellten Kinderschutzregelungen oder -vorstellungen auf der einen Seite und der gelebten Praxis auf der anderen Seite. Auch in Bezug auf die Kooperation mit zuständigen Stellen der Kinder- und Jugendhilfe gibt es Hinweise dafür, dass eine klare und effektive Zusammenarbeit nicht ausreichend geregelt ist. Die mangelnde Unterstützung von Familien aus sogenannten sicheren Herkunftsländern ${ }^{11}$ oder derer, die über Drittstaaten nach Deutschland eingereist

11 An dieser Stelle soll auf die politische Instrumentalisierung des Begriffs der sogenannten sicheren Herkunftsländer hingewiesen werden, die laut zivilgesellschaftlicher Organisationen wie Pro Asyl nichts mit der Sicherheits- und Menschenrechtslage in den 
sind, sticht hierbei besonders heraus. So wird das Recht auf Nichtdiskriminierung (Artikel 2 UNKRK) im Kinder- und Jugendhilfesystem verletzt. Zudem werden Kinder in den Unterkünften in die Planung und Umsetzung von Aktivitäten oder Maßnahmen für ihren Schutz sowie in kinderschutzrelevante Entscheidungen wenig einbezogen, womit ihr Recht auf Partizipation (Artikel 12 UNKRK) nicht ausreichend berücksichtigt wird. Insgesamt zeigt sich eine strukturell bedingte Überforderung der Mitarbeiter*innen im Bereich Kinderschutz, die vor allem auf mangelnde finanzielle und personelle Ressourcen, mangelnde Qualifizierung im Bereich Kinderschutz sowie auf mangelnde Klarheit in Rollen und Verantwortlichkeiten zurückzuführen ist.

Bei einer Gegenüberstellung der von mir identifizierten Gewaltformen in den Unterkünften mit der Kinderschutzpraxis fällt auf, dass überwiegend der Schutz vor innerfamiliärer Gewalt im Fokus von Kinderschutzmaßnahmen und -regelungen steht. In Gewalt zwischen Kindern wird ebenfalls interveniert. Auch Gewalt von Mitarbeiter*innen gegenüber Kindern wird teilweise aufgegriffen. Subtilere Formen, wie Gewalt, die in Strukturen verankert ist oder sich in gesellschaftlichen Diskursen widerspiegeln, werden in den Unterkünften offenbar nicht konkret mit dem Thema Kinderschutz in Verbindung gebracht.

Vor dem Hintergrund, dass die Unterkünfte in einen gesamtgesellschaftlichen Kontext eingebettet sind, werfen all diese Beobachtungen Fragen auf, inwieweit Kinderschutz, wie er in der deutschen Kinder- und Jugendhilfe verstanden und praktiziert wird, die Situationen von Kindern in den Unterkünften tatsächlich aufgreifen kann? Ist es angesichts der vielfältigen strukturellen Gewaltformen, die einen Nährboden für andere Gewaltformen darstellen können, überhaupt möglich, ein effektives Kinderschutzsystem in den Unterkunftssituationen zu schaffen? Wie also kann der Bereich Kinderschutz auch die vielfältigen Formen struktureller Gewalt adressieren?

Strukturelle Gewalt und Ungleichheit zu durchbrechen ist eine gesamtgesellschaftliche Aufgabe. So sehe ich die konstruktive und effektive Zusammenarbeit aller gesellschaftlichen Akteur*innen im Bereich Kindesschutz als das größte Potential an, um das Menschenrecht von Kindern auf Schutz vor Gewalt umzusetzen. Hierfür sind eine stärkere und prominentere öffentliche Auseinandersetzung und Priorisierung des Themas und das unmittelbare Handeln aller Verantwortungsträger*innen im Bereich Politik, Praxis, Medien und Wissenschaft notwendige Voraussetzungen.

Vielleicht sollten wir uns hierfür ein Beispiel an den Kindern der „Kinderrevolution" nehmen, die nicht locker lassen, ihr Recht auf einen gewaltfreien und respektvollen Umgang einzufordern, oder an dem Mädchen Elsa, das immer wieder auf ihre Bedürfnisse und Grenzen hinweist. Ein klares und lautes „MANNO STOPP!“

Ländern zu tun habe, sondern auf Abschreckung und Abschiebung von Schutzsuchenden aus den jeweiligen Ländern abzielen (vgl. Pro Asyl 2018). 
Kinder verfügen über Fähigkeiten und Kompetenzen, auf Formen von Gewalt, mit denen sie konfrontiert sind, hinzuweisen und sich vor Gewalt zu schützen bzw. sich gegen Gewalt zu wehren. So muss vor allem der Anerkennung und Stärkung von Kindern als kompetente Akteur*innen für ihren Schutz vor Gewalt ein besonders hoher Stellenwert eingeräumt werden. Gleichzeitig gilt es, Eltern, die eine zentrale Ressource für den Schutz vor Gewalt von ihren Kindern darstellen, durch Unterstützung und Entlastung zu stärken.

Abschließend lässt sich aus den Ergebnissen dieses Beitrags folgende zentrale Forderung ableiten: Die staatlichen Verantwortungsträger*innen der Bundesrepublik Deutschland müssen umgehend ihrer Verpflichtung nachkommen, das Menschenrecht von Kindern auf Schutz vor jeder Form von Gewalt (Artikel 19 UNKRK), für alle Kinder, die in Unterkünften für geflüchtete Menschen leben, umzusetzen. Die Berücksichtigung des Rechts auf Nichtdiskriminierung (Artikel 2), des Rechts auf Berücksichtigung des Wohles des Kindes (Artikel 3 $\S 1$ ), des Rechts auf Leben, Überleben und Entwicklung (Artikel 6) und des Rechts auf Berücksichtigung der Meinung des Kindes (Artikel 12) muss hierbei gewährleistet sein. Der Frage, wie ein Staat Schutz bieten kann, wenn er selbst für die Aufrechterhaltung bzw. Schaffung gewaltvoller Strukturen wie der geschilderten Unterkunftsformen für geflüchtete Kinder verantwortlich ist, muss in meinen Augen bei der Frage nach der Umsetzung dieser Forderung besondere Bedeutung beigemessen werden.

Als besonders relevant für die Umsetzung der oben genannten Forderung resultieren aus der Analyse des Datenmaterials folgende konkrete Handlungsempfehlungen:

- Maßnahmen zur dezentralen Unterbringung geflüchteter Menschen in Wohnungen umsetzen, die Schließung von Notunterkünften vollständig umsetzen und mittelfristig auch Erstaufnahme- und Gemeinschaftsunterkünfte abschaffen.

- Angebote der Kinder- und Jugendhilfe explizit und proaktiv geflüchteten Familien, insbesondere auch denen mit einer ,unsicheren Bleibeperspektive“" zur Verfügung stellen.

- Partizipationsmöglichkeiten für Kinder insbesondere in Bezug auf kinderschutzrelevante Entscheidungen, Aktivitäten und Maßnahmen in den Unterkünften schaffen und Beschwerdestellen und -mechanismen für Kinder in den Unterkünften einrichten.

- Unterkunftspersonal durch die Bereitstellung höherer personeller und finanzieller Ressourcen entlasten und durch die Qualifizierung und Sensibilisierung im Bereich Kinderschutz und Antidiskriminierung stärken.

- Bundesweite verbindliche Standards für einen umfassenden Gewaltschutz von Kindern in den Unterkünften einführen und eine Begleitung ihrer Umsetzung durch qualifiziertes Personal sicherstellen.

- Eine konstruktive Zusammenarbeit und Vernetzung aller relevanten Akteur*innen im Bereich Kinderschutz fördern und insbesondere die 
Kompetenzen von bereits erfahrenen Trägern im Bereich Kinderschutz für die Unterstützung der Kinderschutzarbeit in den Unterkünften nutzen.

- Eine öffentliche Auseinandersetzung mit einem Kinderschutzverständnis anregen, welches die Agency von Kindern anerkennt und stärkt und ein umfassendes und mehrdimensionales Verständnis von Gewalt berücksichtigt.

- Forschungsinitiativen unterstützen, die vielfältige und vielschichtige Formen von Gewalt, denen insbesondere junge Menschen mit Fluchthintergrund in Unterkünften für geflüchtete Menschen ausgesetzt sind, tiefer analysieren und Wechselwirkungen mit anderen Menschenrechtsverletzungen aufzeigen.

\section{Literatur}

Alanen, Leena (2005): Kindheit als generationales Konzept. In: Hengst, Heinz \& Zeiher, Helga (Hrsg.): Kindheit soziologisch. Wiesbaden: VS Verlag für Sozialwissenschaften/GWV Fachverlage, S. 65-82.

Alberth, Lars (2013): Body Techniques of Vulnerability. The Generational Order and the Body in Child Protection Services. In: Hum Stud 36 (1), S. 67-88. DOI: 10.1007/s10746-013-9259-z.

Bell, John (1995): Understanding Adultism. A Major Obstacle to Developing Positive Youth-Adult Relationships. Hrsg. von. YouthBuild USA.

Berufsverband der Kinder- und Jugendärzte e. V. (BVKJ) - Landesverband Brandenburg und Fachstelle Kinderschutz im Land Brandenburg/Start gGmbH (2016): Früherkennung von Gewalt gegen Kinder und Jugendliche. Brandenburger Leitfaden. Erkennung, Fallmanagement, Interdisziplinäre Hilfesysteme. 6. Auflage. Potsdam und Hennigsdorf.

Breuer, Franz (2003): Subjekthaftigkeit der sozial-/wissenschaftlichen Erkenntnistätigkeit und ihre Reflexion: Epistemologische Fenster, methodische Umsetzungen. In: Forum Qualitative Sozialforschung 4 (2).

Brooks, Robert \& Goldstein, Sam (2017): Das Resilienz-Buch: Wie Eltern ihre Kinder fürs Leben stärken. Das Geheimnis der inneren Widerstandskraft. Stuttgart: Klett-Cotta.

Flasher, Jack (1978): Adultism. Adolescence. 13 (51): 517-523.

Fröhlich-Gildhoff, Klaus \& Rönnau-Böse, Maike (2009): Resilienz. München: Reinhardt.

Galtung, Johan (1969): Violence, Peace, and Peace Research. In: Journal of Peace Research 6 (3), S. 167-191, http://www.jstor.org/stable/422690 (30.11.2018).

Galtung, Johan (1990): Cultural Violence. In: Journal of Peace Research 27 (3), S. 291-305, http://links.jstor.org/sici?sici=0022-3433\%28199008\%2927\%3A3 \%3C291\%3ACV\%3E2.0.CO\%3B2-6 (30.11.2018). 
Gugel, Günther (2006): Gewalt und Gewaltprävention. Grundfragen, Grundlagen, Ansätze und Handlungsfelder von Gewaltprävention und ihre Bedeutung für Entwicklungszusammenarbeit: Institut für Friedenspädagogik Tübingen e. V.

Hengst, Heinz; Zeiher, Helga (2005): Von Kinderwissenschaften zu generationalen Analysen. Einleitung. In: dies. (Hrsg.): Kindheit soziologisch. Wiesbaden: VS Verlag für Sozialwissenschaften/GWV Fachverlage, S. 9-23.

Hengst, Heinz; Zeiher, Helga (Hrsg.) (2005): Kindheit soziologisch. Wiesbaden: VS Verlag für Sozialwissenschaften/GWV Fachverlage GmbH.

Herriger, Norbert (2006): Empowerment in der Sozialen Arbeit. Eine Einführung. 3., erweiterte und aktualisierte Auflage. Stuttgart. W. Kohlhammer.

Honig, Michael-Sebastian (1999): Entwurf einer Theorie der Kindheit. Erste Auflage. Frankfurt am Main: Suhrkamp.

James, Allison \& James, Adrian L. (2008): Key Concepts in Childhood Studies. Los Angeles, Calif.; London: SAGE.

Johansson, Barbro (2012): Doing adulthood in childhood research. In: Childhood 19 (1), S. 101-114, DOI: 10.1177/0907568211408362.

Liebel, Manfred (2013): Kinder und Gerechtigkeit. Über Kinderrechte neu nachdenken. Weinheim und Basel: Beltz Juventa.

Maywald, Jörg (2009): Die UN-Kinderrechtskonvention. Ihr Umsetzungsstand in Deutschland im Bereich des Kinderschutzes. In: Deutsches Jugendinstitut e. V. (Hrsg.): UN-Kinderrechtskonvention. Impulse für den Kinderschutz. IzKKNachrichten 1, S. 4-9.

Pro Asyl: „Sichere Herkunftsstaaten“, https://www.proasyl.de/thema/von-wegensicher/ (Zugriff 3.7.2018).

Reuter, Julia (2002): Ordnung des Anderen. Zum Problem des Eigenen in der Soziologie des Fremden. Bielefeld: transcript.

UNICEF \& Bundesministerium für Familie, Senioren, Frauen und Jugend (BMFSFJ) (2017): Mindeststandards zum Schutz von geflüchteten Menschen in Flüchtlingsunterkünften. Berlin.

United Nations (2011): General comment No. 13. The right of the child to freedom from all forms of violence. Nicht offizielle deutsche Übersetzung: Allgemeine Bemerkung Nr. 13. Das Recht des Kindes auf Schutz vor jeder Form von Gewalt. UN-Kinderrechtskonvention, Artikel 19. Hrsg. von der Stiftung Kinderschutz Schweiz und Kinderanwaltschaft Schweiz, http://www.humanrights.ch/ upload/pdf/130813_CRC_General_Comment_13.pdf (Zugriff 25.10.2017).

Weltgesundheitsorganisation $(\mathrm{WHO})$ (2002): $\bar{W}^{-}$orld Report on Violence and Health. Geneva.

Weltgesundheitsorganisation (WHO) (Hrsg.) (2003): Weltbericht Gewalt und Gesundheit. Zusammenfassung.; Herausgegeben von der Weltgesundheitsorganisation (2002) unter dem Originaltitel: World Report on Violence and Health: Summary. 



\section{Entering the field as researchers and leaving as "aunties": Field relations with young children and their families in a refugee reception centre}

Penelope Scott

“Hello, good morning, please come to my house. I haven't seen you. I love you". Farah, via WhatsApp, 1.8.2017

\section{Introduction}

The WhatsApp voice message from six-year-old Farah was left on the researcher's phone six months after the project fieldwork had ended. It stirred affection for the little girl while evoking vivid memories of the fieldwork, and vexed emotions concerning the indignity of young children living in a crowded reception centre. The message's emotional resonance validates Stebbin's (1991) question whether researchers ever leave the field due to the often enduring emotional consequences of fieldwork. Farah's invitation to come to her house also prompted reflection on the reasons why, even though the fieldwork was over, the researchers ${ }^{1}$ continued to visit her and the other children who had participated in the project.

This chapter elucidates issues and events in the formation of relational ties, during eight months of fieldwork, that led to this unanticipated outcome. By drawing on data from ethnographic fieldnotes, it describes first, how field relations with the children and their families - particularly the mothers - developed during the fieldwork. A specific focus is the ways in which the researchers established a rapport with the children, and how the ensuing relationship was co-constructed through children's choices, parental agendas, and the researchers' various roles as playmate, mediator and the provider of physical and emotional care. However, the portrayal of this relationship reveals its inherent tensions, and how in a research setting characterised by structured disadvantages the relationship proved disruptive in the field. While the researchers'

1 The term 'researchers' refers to the researcher and research assistant who conducted the fieldwork in the reception centre. 
relational ties to the children constituted social capital in the form of social support for both the children and their mothers, the relationship produced ethical dilemmas, for example, by inadvertently contributing to competition among the children and their peers for the researchers' time and attention. Second, the following description of field relations with children in the politically constructed space of a reception centre illustrates the entanglements of ethical principles and moral emotions of anger, frustration and empathy causing the researchers to re-consider their exit strategy from the field.

A central argument of the chapter is that preschool refugee ${ }^{2}$ children's double vulnerability as young children and asylum seekers residing in accommodation centres raises specific issues for researchers. While building rapport is a crucial part of the research relationship, the emotional ties that may develop over time require negotiating ethically appropriate ways of disengaging from the field in order to minimise distress to the children. In developing this argument, section two situates the epistemological significance of field relations within ethnography and contextualises the unique challenges of research with young children living in a refugee reception centre. Section three describes how we built rapport with the children and mothers, as well as the various roles and tasks we performed in the field that earned us the status of "aunties". The fourth section details the formal ending of the project, and the reflexive process leading up to our decision to continue visiting the children when the fieldwork was over. The section concludes with a description of our re-entry into the field and the shift taking place in our relationship as the children employ new modalities of communication.

\subsection{Ethnography, field relations and knowledge production}

Constructivist approaches to ethnography are predicated on the assumption that reality is constructed through human interaction, perceptions and experiences of the social world (Denzin \& Lincoln 2011; Howell 2013). Within this interpretivist ethnographic tradition, social relations between the researcher and participants are central to the ethnographic enterprise of generating thick descriptions of the research setting and its distinguishing social processes. As such, the researcher and participants are viewed as co-constructors of the data

2 The word refugee is used here in its generic sense to refer to individuals fleeing persecution and conflict. This term is to be distinguished from the legal categories asylum seeker, which describes individuals who have submitted a claim for protection in another country and refugee, which refers to the legal status of persons whose application for asylum has been successful. 
produced. However, social relations in the field are fluid and multi-faceted; they are (re)-negotiated in the various spatial contexts of participants' daily lives. These contexts influence the form and content of these relations, and subsequently the data produced.

In the research setting of the reception centre, relations with the children extended into public spaces such as the "kindergarten"3 and the backyard, as well as the private, domestic space of the family room. Here, we also interacted with other family members, particularly the mothers. Charting the course of relations with participants across these spatial domains is instructive, first, as data sources that provide insights into the events and social interactions comprising their daily lives. Second, attention to intersubjectivity in the field makes visible how relations between the researcher and participants are co-constructed, and the ways in which the researcher becomes incorporated into participants' social world. A nuanced presentation of these relationships, and the various roles assumed by the researcher in the field show how "ethnographic 'facts' are built up in the course of everyday participation in the life of the community" (Scheper-Hughes 1992: 25). This transparency facilitates the reader in evaluating the knowledge claims and conclusions drawn from the data.

The process of reflexivity is integral to the negotiation of social relations in the field. Reflexive practice requires consistently attending to the context of knowledge construction, particularly the effects of the researcher, in every phase of the research (Guillemin \& Gillam 2004). Being reflexive entailed reflecting on our positionality, and how our backgrounds as middle class, academically educated adult females, and our ascribed social identities as a 'foreigner' and a member of a minority ethnic group respectively, mediated our relationship to the field and the participants. It further involved questioning the influence of our own socio-cultural constructions of childhood on this relationship. While we were mindful that our multi-layered social biographies and socio-cultural embeddedness filtered our experiences in the field, this awareness also caused us to reflect on the diversity among the children, and the significance of unique aspects of their individual biographies in everyday experiences. Engaging with reflexivity in this way is consistent with constructivist ethnography's ontological assumptions concerning the existence of multiple, socially constructed realities in any social world. The process prompted critical reflection on the ways the children's subjectivities are shaped by developmental discourses relating to birth order and perceived age-related competencies, for example, and socio-legal discourses on their status as asylum seeking minors. This form of reflexive practice was important in helping us position ourselves in relation to each of the children. Importantly, it facilitated enquiry into the children's engagement with their material and social worlds by

3 The centre staff referred to a supervised playroom for children as the "kindergarten". 
acknowledging the complex and individual ways social actors relate to their environment. Taking such a reflexive approach to field relations has a crucial role in eschewing the construction of knowledge that reproduces essentialist or homogenising representations of the 'refugee child'.

\subsection{Contextualising field relations with refugee children}

Mediating our social relations with the children was a recognition of their double vulnerability as children and refugees in search of asylum in Germany. The use of the concept vulnerability in research studies has been criticised for example, for categorising or attaching a label to certain groups or persons without considering the various conditions and relational contexts creating this vulnerability (Luna 2009). However, children's vulnerability is evident in several ways. First, their vulnerability can be considered inherent owing to their "limited knowledge and physical weaknesses" that lead to their dependency on adults (O'Reilly et al. 2013: 31). Second, as Punch (2002) notes, children's vulnerability arises from their marginalisation in adult dominated society; consequently, they experience unequal power relations with adults in their lives. Third, because of a lack of economic and political power children are structurally vulnerable.

For the children participating in the project, this structural vulnerability was further entrenched by their status as asylum seekers and their consequent sociospatial exclusion. The unique social arrangements stipulated by law for asylum seeking children and their families are characterised by their accommodation in crowded centres, welfare provisions in the form of goods, vouchers or cash, and restricted access or barriers to accessing a range of social provisions such as health care and early childhood education (see chapter by Wihstutz "Mittendrin und außen vor"; Bonewit 2016; Wendel 2014). The frugality and inadequacies of their material conditions are compounded by a lack of social contacts. Their social isolation is constructed in part through the biographical disruption caused by flight from conflict, and the attendant physical separation from family, friends and other significant persons comprising their social networks. Furthermore, many refugee accommodation centres are located in remote areas or in districts where anti-foreigner sentiments dominate, which consequently inhibits the formation of social ties.

These socio-economic circumstances create a significant structured disadvantage for children and their families because social networks are regarded as important sources of social capital for immigrant groups by providing them with access to social support (Ryan et. al 2008). Social support encompasses functions performed for an individual by significant others, such as family members and friends and can take the form of emotional, informational, and/or 
instrumental assistance. These forms of support provide the "satisfaction by others of one's needs for affection, esteem, identity, security, and assistance" (Pearlin \& Bierman 2013: 331). In practical terms, the families' social isolation meant that regarding certain forms of social support, such as child care, they were dependent primarily on the creation of ties with other residents in the reception centre. However, the level of social capital in the form of support available to families through these 'horizontal' ties is open to question. Social ties may be most effective in terms of support when they are 'vertical', resulting in access to those who have more resources and knowledge (Bourdieu 1986). Viewed from this perspective, the researchers' structural position in the field was marked by the possession of a greater volume of economic capital such as material assets, cultural capital in the form of contextually relevant knowledge and skills, social capital constituted by social networks and importantly, the symbolic capital afforded by ownership of a secure residence status in Germany. These structural differentials between the researchers and participants underscored the operant power relations underpinning interactions in the field. A recognition of the asymmetry inherent in our relationship strengthened the conviction that the principle of beneficence, "the ethical obligation to act for the benefit of others" (O'Reilly 2013: 49), was critical to our engagement with the children. This principle guided the terms of the children's participation in the research by promising parents and children during the informed consent procedures that while spending time with the children, the researchers would also bring along play resources and creative material. Practically, this meant that the research would "add value" to the children's lives rather than treat them as "data sources" (Kaukko et al. 2017: 20). However, our relationship with the children and their families would reveal the different permutations of acting for the benefit of others in the resource poor context of a refugee reception centre.

The following sections portray the social relations between the researchers and the five children from three families participating in the project: Farah, and two pairs of sisters Taban and Alima, and Saba and Razia. Relations with their mothers are also described because as social actors, and as the primary gatekeepers to the children, their intentions and behaviours influenced our interactions with the children. Given the importance of friendships and contact with peers among preschoolers (Parker et. al 2006), and the relational nature of children's daily experience of the centre, references to their playmates Zarin, Elham, and Nahal are also included. 


\subsection{Building rapport with the children}

Gaining access to the children's social world within the reception centre was a principal aim of the fieldwork. This was contingent on accomplishing field ${ }^{4}$ entry, which is crucial to establishing membership status and an insider perspective, two fundamental goals of ethnography as an interpretive method (Corsaro \&Molinari 2008). Within the setting of the reception centre, achieving these goals was dependent not solely on enacting the formal ethical research procedures of negotiating informed consent with the research participants: the children, their parents and the centre management. It further involved acquiring knowledge of the centre's organisational structure and physical layout, the interpersonal relationships comprising the children's familial and extended networks, and importantly, building rapport with the children. Gaining their trust was, therefore, a necessary part of establishing rapport and developing a relationship with them. As Kellett (2010: 85) notes, "children are unlikely to behave normally", if they are fearful, suspicious or scornful of adult researchers. Yet there has been a tendency in the methodological literature to focus on the subject of ethics in research with children, and other research issues, such as rapport building, are often not discussed because they are considered the same as those with adults (Punch 2002).

Many childhood ethnographers, particularly those doing participant observation with young children, have assumed or adapted a "friend role" (Fine \& Sanstrom 1988) or "least-adult role" (Mandell 1991) in their field relations with children (Gansen 2017). These roles, and their variations, position researchers as atypical, non-authoritarian adult participants in research contexts with children. However, such roles have been critiqued for not acknowledging the complexity of similarities and differences between adults and children (Christensen 2004). Additionally, as Punch (2002) notes, when doing participant observation with children, adults can never really become full participants in their social worlds because they can never truly be children again.

A challenge we faced in efforts at rapport building was the existence of a language barrier. The children participating in the project - as well as their playmates - were from multi-ethnic, multi- lingual Afghanistan, and we did not speak their family language. Though staff in the kindergarten addressed the children in German (see chapter by Scott \& Le), their comprehension level and oral skills in German varied. Therefore, we had to be reflexive about our communication style. Given that non-verbal cues are just as important as choosing appropriate words in everyday interactions (Knapp et al. 2014), we were mindful of the use of facial expressions, eye contact or gestures such as head nods, as well as the tone and volume of our voices while communicating

4 The 'field' is a metaphor for the setting and/or population where and/or with whom the researcher conducts fieldwork, i.e. data collection. 
with the children. We were equally vigilant about the children's responses, their non-verbal behaviours and unique forms of communication, which we regarded as important indicators of their assent (Cocks 2006) when spending time with us.

We also ensured that when we visited them, we adhered to the practice of individually greeting the children and saying farewell to each of them by their name. Although this is customary polite behaviour, the generational ordering of society, which does not accord children the same social status as adults, manifests in children being treated differently. As a result, children are often treated patronisingly, or are overlooked in adult society thereby rendering them invisible. Therefore, by initiating and ending our interactions with the children in specific ways, we sought to acknowledge their personhood and so reaffirm our relationship with them. Our consistent, intentional actions in this regard proved to be rewarding relationship building practices. Soon, little greeting and farewell rituals, initiated by the children, developed as we arrived or departed. Whenever we were about to leave Taban and Alima's family room for example, they would each shake our hand and while holding our hand, they jumped up and down and counted from one to ten in German.

Our efforts at relationship building with children were also guided by observing research practices, that as Christensen (2004: 166) notes, take account of "children's experiences, interests, values and everyday routines". In the early phase of the fieldwork we adapted to the children's routines, for example, by fitting into the setting wherever we found them, and being mindful not to intrude into their child spaces. If they were playing and did not notice our arrival, we did not interrupt them but waited until they approached us, or until there was a break in their activity before we greeted them. If the children chose to hang around us, which usually happened when we encountered them in the backyard or in the main building, we engaged them in conversation. Considering their limited German language skills, we asked simple questions such as "did you have fun in the kindergarten? Who did you play with today? How is your baby sister?" The questions centred on their everyday experiences with places, persons and objects familiar to them, and used vocabulary items they most likely would have heard in the kindergarten. These questions sometimes elicited monosyllabic responses, such as "yes", "no", "good", "not good", or smiles and giggles as the children struggled to communicate. On occasions they spoke to us in their own language. In some instances, the questions we asked or simple instructions we gave while doing activities together were met with blank stares suggesting to us that they probably had not understood.

Although responding in German may have been challenging for the children, they initiated interactions and reciprocated our interest in ways that demonstrated how adept they are at non-verbal communication. Sometimes they played with or stroked the researchers' hair or pulled us by the hand towards the backyard or the sand pit if they wanted to play there. The children 
usually gazed curiously at our rucksacks, and sometimes touched or peered into them inquisitively, indicating their interest to know what we had brought with us. They reacted with enthusiasm when they saw the creative activities and material, such as the mosaic set, colouring books, drawing paper, crayons and modelling clay that we had. The children soon showed a strong preference to occupy themselves creatively with the activities and material when we visited. This was unsurprising in certain respects considering the limited availability of such resources in the kindergarten (see chapter by Scott \& Le). Sometimes the children appropriated the material and found inventive ways of using it to suit their own interests: Razia took delight in hammering the floor with the colouring pencils until the points broke, or disassembling the sharpener from its removable cover, filling it with water and sprinkling it over the floor. On occasions, the children or their playmates appropriated the material by trying to smuggle it away in their pockets.

Crucial to building rapport with the children in this formative stage - and maintaining it during the fieldwork - was respecting their wishes regarding how they spent time with us. This required surrendering adult power to be constantly in control of organising and directing their activities. As such, the relationship that developed was co-constructed through the ways in which the children related to us and positioned us in their social world. The following incident, which occurred a few weeks after the fieldwork started, illustrates the dynamics of our interactions and how children negotiated their relationship with us:

The kindergarten is closing, and before Taban and Alima leave I ask them if they would like to play. They enthusiastically say "yes". We go outside into the backyard, and Farah, who probably overheard the conversation, follows us [...] I then ask if they want to colour and they all said "yes". As I open my rucksack to bring out the drawing paper, colouring pencils and wax crayons, they all chant loudly "chocolate!", "chocolate!" I had some chocolate filled biscuits and offer each child one. They settle down and begin drawing and colouring. Taban starts singing and the other two children appear content as they concentrate on their drawings. [...]. Farah takes a red wax crayon and tries to colour her lower lip with it, as if she is applying lipstick. Immediately, Taban starts laughing, she selects a red colouring pencil and tries to do the same. [...]. Taban giggles, leans forward and points to my lips as if she wants to draw attention to the lipstick I'm wearing (Field visit 8, 11.7.2016).

The little girls' playful demand for "chocolate" was an opportunistic tactic aimed at influencing the researcher to comply with their wishes. It provides insight into some of the ways their behaviours sought to invert the adult-child power relationship. On previous visits we had offered the children biscuits, but usually shortly before we left. However, on this occasion, they collectively exercised their 'pester power' to challenge this adult imposed routine by demanding a snack at the beginning of our time together. Rather than insist that 
the children wait, which would have enacted adult authority and produced the children's submission, the researcher complied with their request. This tacit approval of their behaviour minimalised the power inequalities in our relationship and may have encouraged the next phase of our interaction. By re-enacting a practice adopted from the adult world, the children created a fantasy play routine, with the researcher seemingly a model for their little game of 'lipstick application'. On one level, this interaction signals the ways children appropriate researchers, and use their presence to their own advantage by including them in play activities (Gallacher \& Gallagher 2008). On another, the exchange captures the subtle ways in which the researcher and child participants collectively produced the researcher's participant status in the research setting.

\subsection{Roles and tasks in the field: mediating squabbles, drying tears and wiping noses}

While developing rapport was an important trust building mechanism, our social relations with the children - and their families - were also constructed through different roles we assumed and tasks we performed in the field. Our researcher role was, therefore, in constant flux as we responded to participants' actions and expectations. One such role involved intervening and mediating in physical conflicts during their unsupervised free play. The children had time restricted access to the kindergarten, but they had their own temporal strategies for creating play spaces outside of the kindergarten. When it closed at 12:30, for example, which marked the beginning of a lunch break in the centre's organisational routine, children often went into the backyard or hung around in the reception area to play until a parent or an older sibling came looking for them. In these unsupervised play spaces, conflicts sometimes occurred:

I walk into the backyard to see if the children are there. Zarin comes running across the sand pit towards me while demonstratively throwing sand in the air and saying in a distressed tone of voice, "Farah is doing this". It sounds as if Farah is throwing sand at the others. I see Farah and Taban at the top of the stairs to the slide, both jostling to see who could or would be first to slide down. Farah hits Taban on her head. Taban screams, hits Farah and grabs her by the hair [...] Farah retaliates by grabbing Taban's hair. They are in a deadlockor headlock - with each grabbing the other's hair while balancing precariously on the stairs. It isn't easy to separate them, and I have to hold Taban, who is standing on the top rung, so she won't fall. I lift her down, then hold out my hand to Farah and help her down the stairs (Field visit 6, 1.7.2016).

Zarin's distress as she recounted Farah's behaviour signalled that the child's actions were not considered playful by her playmate(s). By reporting Farah's 
behaviour to an adult Zarin demonstrated one way in which the children enacted social control over their peers. This act was underpinned by the assumption of adult authority to arbitrate, and importantly, the expectation of adult intervention. It defined a role for the researcher as a mediator in the research setting. Some childhood ethnographers advocate an avoidance of authority or sanctioning children's behaviour in an effort to build trusting relationships with them (Fine \& Sanstrom 1988; Mandell 1991). However, as Edmond (2005) notes, the researcher needs to consider the practicalities of maintaining this position in certain situations because children's physical and emotional safety are of paramount ethical importance. As such, the physical aggression characterising the tussle, and the risk of an accident occurring were grounds warranting intervention.

The time we spent with the children usually involved close physical contact. We were greeted affectionately with hugs, and the children often appropriated our bodies as playthings by jostling with one another to sit on our laps or jumping onto our backs when we sat with them on the floor. However, the physical contact and the interactions in which they were embedded sometimes constituted care work, which Erickson \& Stacy (2013: 176) define as the "work of caring for others". One dimension of care work involved performing physical care tasks they were unable to do independently. If the children needed to use a communal toilet when they were in our charge, for example, we accompanied them. We did so partly out of concern for the children's safety given the known inadequacies of child protection approaches in accommodation centres (Lewek \& Naber 2017; Wagner 2016). It was also necessary because the children sometimes had difficulty using the adult sized toilets and needed help to do so. They did not verbalise or demonstrate resistance to receiving our assistance. Their acceptance of the care we offered signalled their trust in us and the normalisation of us performing such tasks, as the following extract shows:

Saba, Razia and their little brother Abed are alone in the sand pit and each has a bucket filled with sand that they had doused with water. They dig with their hands into the bucket and make different shapes with the wet sand. Saba is sniffling loudly, and snot starts oozing from her nose. As soon as she wipes it off on the sleeve of her blouse, more snot streams out of her nose. I stoop beside her with a tissue in my hand. She buries her face into the tissue and blows, her hands still stuck in the bucket of wet sand. I hear Razia and Abed sniffling and notice that they both have runny noses. I hold a tissue to their faces in turn, they blow into it and then promptly resume playing (Field visit 16, 15.09.2016).

Another dimension of care work characterising our relationship with the children was emotional work. This construct refers to efforts made to have empathy with others and includes feedback and/ or behaviours to alter how others feel, and to create positive emotions in others (England \& Farks 1986; England $\&$ Folbre 1999). At times our emotional work accompanied acts of physical care that also extended to the children's playmates. For example, when Zarin 
sobbed uncontrollably at the sight of red marker stains on her dress because she feared getting into trouble with her mother, the researcher wiped out the stains while soothing the distraught child and reassuring her that the stains would disappear.

There were instances, however, when the children demonstrated behaviours that gave us insights into their emotional worlds, and in turn elicited our emotional feedback in verbal and non-verbal forms. These behaviours occurred alongside transitions and adjustments that altered their sense of identity and status both socially and within their family network. One such adjustment involved the birth of siblings. Over the fieldwork period two of the three participating families welcomed a new baby into the family unit. For four-yearold Alima, the birth of her baby brother coincided with several other changes in her daily life: her only sibling started primary school, she stopped going to the centre's kindergarten and instead, stayed in the family room with her mother and the baby. Over this period, we noticed that Alima reacted more enthusiastically to our visits and her displays of affections towards the researcher became more effusive:

We meet Ms. Sanjari in the corridor [...] and walk together with her to the family's room. She knocks on the door and Alima opens it. The child smiles broadly at us, then she rushes towards me, her little arms outstretched, and hugs me around my legs. [...]. Ms. Sanjari leaves the room [...]. We sit down on the carpet with the girls and watch cartoons on television together while the baby sleeps in his crib. Ms. Sanjari returns and sits with us on the carpet. Alima moves closer to me and rests her arm on my leg. After a few minutes she then crawls onto my lap and lies there, cooing briefly like a baby. I smile at her and stroke her hair. Ms. Sanjari watches us and smiles (Field visit 23, 12.01.2017).

Whatever the reason(s) underlying Alima's display of physical affection towards the researcher, her behaviour appeared to signal a need for attention and emotional warmth. The interaction constituted one of many moments of emotional work in the field when the children sought and/or needed emotional feedback from the researchers, often in the form of recognition and praise for creative activities they accomplished, encouragement if they had difficulty doing so or soothing if they became upset in tussles with siblings or playmates over play resources.

The need for emotional feedback was particularly noticeably among the three older children when they were about to start primary school. This is a significant life event marking an upward shift in children's social status as they transition from preschool arrangements to primary school, and assume a new identity as pupils. For the children in the centre, this liminal phase, in which they were no longer preschoolers but were not yet pupils, was marked by several practices intended to inaugurate them into assuming their new identity. These included standard bureaucratic practices such as a health check, and an introduction to another building on the compound where the school lessons 
took place. ${ }^{5}$ Over this period of impending change in their identity and associated use of physical space, Taban and Farah often excitedly mentioned to the researchers "I'm going to school!" This excitement peaked when the children received a schoolbag equipped with stationery and a pencil case from the centre. The issuing of a schoolbag was another bureaucratic practice intended to prepare them for school. It was an important material symbol of a significant temporal aspect of the children's lives, and they were aware of what it signified. Each of the children proudly exhibited their new schoolbag to us when we visited. Farah smiled broadly, telling us again she was going to start school, as she opened it and took out its contents. She unzipped her pencil case to reveal an array of crayons, and then showed us one by one her collection of notebooks. The children's ceremonious display of their schoolbag solicited our recognition of the important transition they were about to experience. In showing interest and positively affirming the specialness of this event we not only observed this transition but also participated in creating the happiness and excitement associated with it. Such emotional work was part of the fabric of our relationship with the children, and over time, these shared moments of emotional interaction forged an attachment between us. Significantly, this emotional work translated into support for the children and as such, constituted social capital accessible to them through the durable relational ties we shared.

\subsection{Rapport with the mothers}

Establishing a rapport with the children's parents was important. As adult gatekeepers, they regulated access to the children (Punch 2002), and due to their parental caregiving role, which included organising and managing children's daily lives, their views on children's everyday experiences in the centre were relevant to the research aims. Opportunities for rapport building presented themselves during our visits to the family room. On most occasions our interactions were mainly with the mothers; in the two families where the children were accompanied by both parents, the fathers were usually away at German classes. The mothers invariably invited us into the family room, which was a particularly hospitable gesture given how small it was. They treated us like guests by offering us a range of refreshments and often invited us to stay longer and eat with them. The children witnessed the cordial, welcoming way parents received us, which was different to how they interacted with centre staff who came to the family room; staff members did not enter the room but spoke with

5 Due to a shortage of classrooms in local schools, the district school authority organised classes for refugee children known as "Willkommensklassen" (Welcome Classes) in a vacant building on the premises of the reception centre. 
parents at the doorway. The parents' hospitable attitude mediated the children's behaviour towards us in discernible ways and subsequently, how they constructed our place in their relationship network. The children often offered us snacks that their mothers had placed on the carpet where we sat together or brought us our shoes and escorted us to the door when we were about to leave. By observing these hospitality rituals, the children enacted the role of host, and their performative acts positioned the researchers as guests of the family in their social network.

Despite the language barrier, the mothers made efforts to communicate with us and reciprocated when we engaged them in conversation, sometimes relying on their older children who knew some German to translate. We observed, however, that while we interacted with the children, the mothers often occupied themselves in various ways. Ms. Jafari, who was extremely distressed about the fate of two children she was separated from on the journey to Germany, usually slept during the time we spent with Farah. Ms. Sanjari often left us alone in the room with the children while she went to the communal kitchen to perform chores or to the laundry room to attend to the washing. Like Ms. Noorzai, she gave birth over the fieldwork period, and both women often breastfed their baby soon after our arrival. The regularity of this pattern in the mothers' behaviour, regardless of the timing of our visits, suggested that attending to domestic chores and the needs of other offspring during our time with the children were temporal strategies employed by the mothers to micromanage the household - or in the case of Ms. Jafari, to practise self-care. Our visits facilitated this by providing the children with occupation and oversight, important forms of social support for the mothers that emerged as a secondary asset of the time we spent with their children. The following fieldnotes detailing a visit to the room occupied by the Noorzai's - a family with nine children - exemplify how the mother micro-managed her role, and the subtle ways in which the researchers were integrated into the distribution of care of the baby:

When we arrive at the family's room, Ms. Noorzai is there with Razia, her little brother Abed and her baby sister [...]. Ms. Noorzai invites us in and leaves the room. She returns a few minutes later with Saba, and two of her older sisters, Azita and Kimia, who join us on the carpet. [...] Ms. Noorzai sits down on the carpet with us and breastfeeds the baby. Afterwards, she puts the baby on the bed for a nap and goes to the kitchenette, in a corner of the room. The five children are all busy colouring or working with creative material [...]. The baby starts crying and Kimia goes to lie down on the bed beside her. Ms. Noorzai breastfeeds the baby again, but this time puts her down to rest on a large pillow on the carpet beside us. She leaves the room, returns after a few minutes and goes back to the kitchenette [...]. The baby starts crying. Azita picks her up from the pillow, gives her to the research assistant to hold and then resumes colouring. After about 10 minutes, Kimia leaves the room and Azita takes the baby 
from the research assistant and places her back on the pillow. She gets up from the carpet, sits on a bed and begins playing a board game. Abed climbs onto the bed and plays the game with her. Ms. Noorzai strides over to the bed and tells them off in a loud, angry tone of voice. They immediately jump off the bed, and she returns to the kitchenette [...]. The baby is still sleeping peacefully on the pillow. It is time for us to go and Azita and Saba help us pack up while Razia and Abed horse around. [...] the baby awakes, she slides off the large pillow and lands on her back on the floor. In the hustle and bustle of activity in the room no one else seems to notice. She starts to cry, her little arms and legs flailing in the air like a distress signal. I pick her up, cradle her in my arms, and rock her gently to try and soothe her. After a few minutes Ms. Noorzai takes the baby from me and breastfeeds her again (Field visit 21, 07.12.2016).

This domestic scene, constituted by the actors' intentional behaviours and interactions, demonstrates on one level the significance of our visits to Ms. Noorzai. Although only Saba and Razia were recruited as participants in the project, their younger brother Abed and school-aged female siblings usually joined in our activities with the two girls, if they were present in the room. If they were elsewhere in the building when we arrived, Ms. Noorzai would look for them. Her agency enabled the older children's participation in the creative activities and games we brought. This facilitative behaviour also enabled their access to non-material forms of support, such as German language practice, companionship and socialising, that interacting with us offered. These older siblings, as well as the younger ones, looked forward to our visits. As Ms. Noorzai said to us in an interview, the children asked her each week, "when are the aunties coming?"

On another level, the scenario depicts the construction of intrafamilial care relations and how older children as social actors engage in the work of minding younger siblings. However, it also reveals the ways the researchers were enlisted into enacting caring practices, either through the intentional actions of others, or by default due to unmet needs arising in the field. The researchers' incorporation into the distribution of care within the family unit illustrates how the participant status of the researchers became embedded in the family network over time. The social support this provided to both the children and their mothers constituted an important form of social capital that could be mobilised to mitigate the scarcity of social support in the resource poor setting of their daily lives.

\section{4 "Mine!": Competition in the field}

The spaces where we spent time with the children shifted over the course of the fieldwork. At the beginning of the fieldwork in the summer, we observed 
them in the kindergarten with their peers, and outside in the backyard where they played with siblings and playmates. The constellation of their playmate network was relatively stable, and these friends usually participated in our activities with the children whenever we spent time with them in the backyard. There were instances, however, when school-aged children or other preschoolers asked if they could join in - or they spontaneously involved themselves in the activities. At times, the number of children wanting to participate tested our capacity to provide them with sufficient play resources and attention. We felt an ethical obligation not to exclude any of the children, and out of a sense of distributive justice, one of the core guiding principles of ethical research (O'Reilly et al. 2013; see chapter by Tràn), we were committed to sharing the play material we brought with as many of the children as was possible. We noticed, however, that the older children, particularly the boys, had the advantage of being able to monopolise these resources, which consequently pitted them against the younger ones. This created an ethical dilemma. We felt complicit in facilitating competitive behaviours among the children and reproducing conditions similar to those obtaining in the kindergarten, where children often had to compete for the limited available play equipment. However, actively excluding the children's playmates or other children wanting to play was not an ethical alternative.

An unexpected resolution to this dilemma soon occurred. In late summer, possibly due to weather changes and/or parents restricting the children's spatial autonomy because of anxieties about their safety, we often did not find them in the backyard. Instead, they were in the family room, where we usually spent time with them - if that was what the children and their parents preferred. Once we started visiting the family room, however, we observed that when we did encounter the children playing in the corridors, backyard or kindergarten, they interrupted their play and invariably expressed the preference to spend time with us in the family room. The children would escort us by the hand, sometimes pulling us away from other playmates or children who tried to get our attention.

We interpreted the children's actions as defensive behaviours influenced by living arrangements that undermined playful engagement with their environment and offered limited opportunities for developing meaningful ties with adult significant others. The social and material deprivation characterising their lives in the crowded reception centre created conditions in which children had to compete with one another for available material and non-material resources (see chapter by Scott \& Le). Their possessiveness of us, therefore, was functional as it served to protect their own personal play spaces with access to activities, attention and forms of support that time with us provided. However, an incident occurred in the backyard that caused us to reflect on how our presence impacted upon the children's behaviour towards each other: 
Taban, Alima, Farah and Elham are among a group of children standing outside the kindergarten. Taban and Alima immediately run up to me and hug me around my legs. Farah approaches me and holds my hand tightly. Elham comes forward and tries to hug me too. Suddenly Farah gently but firmly pushes her away and says "Mine!" with a scowl on her face. Taban says "No, mine!" Taban and Alima hold hands and then hold mine forming a circle. Farah moves into the middle of the circle. Led by Taban and Alima, we walk briskly around Farah in circles, as if we are playing ring around the roses. Razia suddenly appears, she grabs me by the hand with a strength that surprises me. She pulls me out of the circle and leads me by the hand to the entrance of the building. Her little chest is heaving from the exertion and she looks up at me with wideopened eyes. I smile at her and am overwhelmed by strong protective feelings. I try to console her by saying that I'll be coming again to visit her next week. I don't know if she understands. I leave wondering what part I, and the research project, have played in this display of competitive behaviours (Field visit 15, 8.09.2016).

One week later:

When I arrive at the family's room Razia is already standing at the half-opened door staring into the corridor. She greets me with a big smile and says loudly "Mine!" (Field visit 16, 15.09. 2016).

The competitive attitude of the children, verbalised by Farah, Taban and Alima, and given palpable expression by Razia's intervention, crystallised two issues pertaining to the effects of our presence in the field. First, while their behaviour confirmed the significance of our visits to the little girls, it also showed that our time with the children had inadvertently contributed to entrenching rivalry among them. We had become another object of competition in the field. Second, Farah's possessive actions resulting in the exclusion of her friend Elham from our game prompted reflection on how our time with the 'project children' was perceived by their peers. They knew we visited the children in the family room. Their playmates sometimes followed us to the room, and on occasions the mothers allowed some of the children accompanying us, particularly Zarin, Elham, and Nahal to stay. Our visits to the family room however, appeared to create expectations among the children's playmates that we would visit them in their family room too. Their playmates, particularly Zarin, Elham, and Nahal, frequently asked - and sometimes even begged - us to do so. Our polite refusals did not deter them from repeatedly inviting us, which indicated that such visits were perceived as special and consequently, highly desired by the children. This insight was accompanied by an awareness that the research project, with the benefits that our time with the participating children offered, in effect privileged them in the perception of their peers. However, it was a privilege from which they were excluded. This interpretation solidified when we sometimes encountered Elham loitering in the corridor outside of Taban's or Farah's family room as we were leaving. 
This experience with the playmates was foremost an indication of the great need among preschool children in the centre for play spaces in which they could occupy themselves with creative pastimes, and create ties to significant others through social interaction. However, the researchers' decision to respect the children's preference to spend time in the family room unwittingly contributed to the creation of 'exclusive' and 'exclusionary' play spaces that were, in most instances, out of bounds for their playmates. The dilemma this posed, together with the competitive behaviours it produced, exemplifies what Bell (2014: 5) describes as "the impossibility of pre-empting the course that ethnographic fieldwork might take". The emergent situation proved a salutary reminder of the ways in which the researcher's engagement in the field disrupts it, and that the ethical imperative to do no harm is partly aspirational. Not all contingencies can be anticipated or planned for and predicaments occur for which there is no immediate, satisfactory solution. However, describing the 'ripple effects' of the researcher's participant status in the field is crucial first, to providing transparent portrayals of the complexity and 'messiness' of the social world we seek to study. Second, discussing these effects provides opportunities for reflexive engagement with the difficult trade-offs researchers may have to make in prioritising ethical obligations to their research participants in the field.

\subsection{Exiting the field}

Withdrawal from the field carries responsibilities to research participants and requires careful structuring and planning (Edmond 2005; Stebbins 1991; Taylor 1991). However, such planning is fraught with uncertainties in research with asylum seekers due to the ever-present threat of a rejection of their application and consequent deportation. The timing of the termination of the fieldwork was communicated during the informed consent procedure, but we were aware that we may have to adjust our plans to suit the families' circumstances if their applications were rejected. These anxieties fortunately proved unfounded as all the families received a positive outcome to their application shortly before the fieldwork ended.

We subsequently arranged our official exit from the field by organising a meeting with the parents and the children, attended by an interpreter, in which we thanked them for their participation, gave them a de-briefing on what we had learned during our research and provided them with the opportunity to ask us questions. In planning this event, a key consideration was how to deal with two challenges: first, how to communicate the impending end of the fieldwork in a concrete way to the children, and second, how to manage the close personal relationship, which had developed with them, once the fieldwork was 
over. As Stebbins (1991: 248) writes, "Leaving the field is not simply a matter of wrapping up a study, but of dealing with a change in how one relates to the people one has studied". However, as Iversen (2009) notes, in the ethnographic literature, scant attention is paid to "getting out" of the field, and little guidance exists on how to disengage sensitively from a field with vulnerable young children living indefinitely in refugee accommodation at such a crucial stage of their cognitive, social and emotional development.

We had anticipated the first of these challenges before the fieldwork and planned for it in a very specific way. As an activity, the children decorated a folder with a picture we had taken of them and a selection of different stickers and coloured tape. With our help, they also wrote their name on the cover. We collected the pictures they drew or coloured and placed them in the folders at the end of each visit. The folders were a portfolio of their creative achievements and served as a material symbol of our time together. During the farewell meeting, as we explained that our weekly visits were about to end, we presented each child with their folder. They reacted enthusiastically when they received it and immediately studied the pictures with obvious delight. As a way of showing our appreciation for their participation in the project each child also received a voucher for a local department store where they could purchase a toy, game, or educational material of their choice.

The challenge regarding how best to manage our relationship with the children emerged as an issue requiring serious consideration soon after the fieldwork started. Not only did genuine, reciprocal affection develop between us and the children, we also soon realised how needy they were for attention and play activities. As Taylor writes (1991: 246), research with vulnerable participants places certain obligations on researchers once the fieldwork ends:

"[...] when we cultivate close personal relationships with informants, we sometimes incur an ongoing responsibility to them. (...) some of the people we study are vulnerable and lonely and come to depend on us. Whether by phasing out of the relationship gradually, helping the person to find other friends, or staying in touch, it seems that we owe something to people whom we have encouraged to become close to".

But what did we "owe them" and what responsibilities did we have? A critical aspect of the reflexive process concerning our post-fieldwork relationship to the children was an awareness of a range of moral emotions, particularly frustration, anger, and empathy, that had permeated our subjective experiences of the field. Such affective states have a productive role in fieldwork as they are conduits to understanding phenomena in the research setting (Davies 2010). Punch (2012) notes further, that contemplating emotional reactions to issues and situations in the field helps the researcher understand the daily challenges participants face. Additionally, by "reflecting inward as well as observing 
outward" (Ellis 1991: 125), the researcher can be guided by their emotional states concerning how to act.

The indignities that the children had to bear in everyday life were distressing to witness. Seeing small children struggle to use filthy, adult sized toilets, watching them compete for limited play resources in the kindergarten, and being bystanders to the humiliation they endured as a childcare assistant regularly shouted at them were among some of the field experiences that evoked our anger and frustration. The negative emotional reactions these observations elicited compelled us to analyse the meaning of our responses. In abstract terms, our frustration and anger were a visceral reaction to the violation of a socio-cultural, moral code of autonomy that is valued as an integral aspect of childhood in German society. The ethic of autonomy involves respect for children's rights, freedoms and choices. It is codified into national legislation, stipulated in international conventions and places specific obligations on the state. Yet the discriminatory conditions that the children were forced to live in, and the socio-legal barriers attached to their status as asylum seekers infringed upon these rights and liberties (see chapter by Wihstutz, "Mittendrin und außen vor"). They were trapped in circumstances characterised by structured inequalities and vulnerabilities resulting from unresolved tensions between their social status as children and their political identity as asylum seekers. The feelings of injustice this generated created empathy for the children and produced the moral impulse to act. This reflexive process, featuring entanglements of ethical principles and moral emotions, demonstrates how ethnographic research involves negotiating three modes of engaging with reality: the emotional, the analytical and the political (Davies 2010).

In considering how we could translate our convictions into action we were mindful that we were a significant resource for the children. Over time, we had become integrated into their limited, locally based relationship network. The forms of social support we were able to provide, due to our structural positioning in the field, could mitigate some of the deficits they experienced by living in refugee accommodation. Leaving the field would deprive them for example, of access to the creative material they so much enjoyed, and which contributed to their development. Particularly in view of the emotional attachment between us, our departure and the consequent ending of our relationship had the potential of being another disruption in their lives that the children would have to endure. The likelihood of this causing them distress also featured prominently in deliberations over our ethical responsibilities during our exit and the postfieldwork phase. Consequently, we decided to ask the parents if we could continue to spend time with the children, if the children wanted to, at intervals of every three to four weeks rather than weekly. The parents responded positively to our request and the children eagerly agreed. It was on these negotiated terms that we shed our official role as researchers and took ownership of our assigned identity as "aunties". 


\subsection{Re-entering the field}

Our first visit after this meeting occurred approximately three weeks later and it was evident that the children had noticed the unusually long time since our last visit. Taban asked "why were you away so long?" and Razia pouted when she saw us, which we interpreted as a sign of her displeasure at the length of our absence. However, these initial reactions drawing attention to the change in our visiting routine soon gave way to the usual open and warm interaction that we had shared in the past. The relational pattern co-constructed by us and the children still continues as we follow the children's lead regarding how they choose to spend time with us. Another enduring pattern is the children's continued preference for creative activities such as colouring and playing with modelling clay, though they occupy themselves happily with other games and child-centred activity books and pastimes that we bring.

In the year since the change to our visiting schedule, subsequent to our formal departure from the field, the children have had to make other adjustments. Approximately five months after the fieldwork ended the families were finally moved out of the reception centre and relocated in a purpose-built collective accommodation centre in a more remote district. This was the third accommodation centre the children were moved to in the approximately twentytwo months since their arrival in Germany. We were apprehensive about the effects of the change on the children, particularly given the known association between mental distress in asylum seeking children and residential relocation (Goosen et al. 2014). On our first visit to the children in the new centre we were overwhelmed by their excitement and happiness as we arrived. We interpreted their effusive reactions partly as an indication that our visits represented an important sign of continuity in their young lives amidst the recent tumult of change precipitated by the move.

Since their relocation to the new centre there has been a shift in our relationship. The children have started to use the messaging service WhatsApp on the family smartphone as a medium to initiate contact with us. Farah, Taban and Alima have sent voice messages, and Farah, as well as Sabia and Razia randomly send emoticons or other icons such as footballs or people symbols. The researcher has also found several missed calls from Saba and Razia on her phone. The children's use of digital devices as a communication medium is in certain respects unsurprising given the skills small children develop from an early age in practices related to new technologies (Marsh et. al, 2005). However, the children's agentic act of initiating contact with us is significant in several ways. First, it shows how we have now become integrated into a virtual social network comprising family members and significant others that is spatially dispersed and includes transnational ties. Although this medium of communication is no substitute for the embodied gestures of face-to-face 
interaction, it offers the children possibilities to overcome their socio-spatial isolation. Second, by contacting us the children have demonstrated their capacity to participate in constructing social relationships in their own unique ways, as well as their interest in maintaining ties with us. We interpret this interest also as an indication of the children's need for continued social support, an important protective factor in asylum seeking children's mental and physical wellbeing (Goosen et al. 2014). Our visits will continue as long as the children's need for our support, and interest in spending time with us exist.

\section{Conclusion}

The fieldwork experiences described in this chapter have underscored the intense, relational nature of research with young children spending their formative years in collective refugee accommodation. Given their social status as young children, building a trusting relationship with them requires striving to respect their wishes, being mindful about how power is negotiated with and by children, and responding to their needs by "acting as a "responsible adult" (Christensen 2004: 174). Establishing a rapport with parents is an important aspect of the fieldwork, and in resource poor accommodation centres, where practical forms of social support are likely to be scarce, the researcher's role may become entwined with parents' agendas. The structural conditions affecting both parents and children are a material marker of their political identity as asylum seekers. Other markers defining children's position in the field include their identities as ethnic minorities and non-native German language speakers. As such, it is argued that in the field researchers should engage reflexively with their own ethnocentric understandings of childhood, and consider the inherent challenges children face in their new linguistic environment. The latter task requires researchers to be reflexive about their communication styles and attuned to each child's unique verbal and non-verbal communication patterns.

During fieldwork the attachments that form and the needs that arise may transcend expectation. Therefore, earnest ethical reflection is required to address how these relational bonds should be handled sensitively, and what responsibilities we as researchers have to our participants when the fieldwork ends. These responsibilities are determined by the interplay of intersubjective processes and material circumstances shaping relational ties in the field, and may vary according to the unique biographies of each child. It is then incumbent on us to take stock of our own resources and capacities to find ethical and meaningful ways to respect the trust, affection and time the children have unreservedly given us. 
It is further argued that given the unique living arrangements in accommodation centres, researchers should consider the fieldwork's possible effects on the wider peer network in which formally recruited child participants are relationally embedded. A defining aspect of ethnographic research in accommodation centres is the ways in which the social and the spatial are interwoven in the research setting. In these cramped conditions, where the production of private space is highly constrained, the researchers' engagement with child participants takes place in the public gaze and other young children will seek to participate in the construction of this interaction. Consequently, they become social actors in the field we study and contribute to providing insight into its characteristic social processes. This presents a compelling argument for researchers to consider their ethical responsibilities to the wider group of young children in the accommodation centre, both in the planning and disengagement phases of the fieldwork. Respecting their role in the research process could take the form of allocating budgetary resources for donations of creative material to the childcare facility, and/or the organisation of fun days providing a variety of activities and amusements, for example. In an environment where all young children are subject to the same disadvantaged conditions such measures are important material forms of distributive justice in the field.

\section{Acknowledgements}

My thanks to Professor Dr. Hella von Unger for her helpful comments on an earlier draft of the chapter.

\section{References}

Bell, Kirsten (2014): Resisting Commensurability: Against Informed Consent as an Anthropological Virtue. In: American Anthropologist 116, 3, pp. 1-12.

Bonewit, Anne (2016): Reception of female refugees and asylum seekers in the EU: Case study Germany (Policy Department C: Citizens' Rights and Constitutional Affairs). Brussels, Belgium: European Parliament.

Bourdieu, Pierre (1986): 'The Forms of Capital'. In: Richardson, John (Ed.): Handbook of Theory and Research for the Sociology of Education. New York: Greenwood Press, pp. 241-58.

Christensen, Pia (2004): Children's Participation in Ethnographic Research: Issues of Power and Representation. In: Children \& Society 18, pp. 165-176. 
Cocks, Alison (2006): The Ethical Maze - Finding an inclusive path towards gaining children's agreement to research participation. In: Childhood 13, 2, pp. $247-$ 266.

Corsaro, William \& Molinari, Luisa (2008): Entering and observing in children's worlds: A reflection on a longitudinal ethnography of early education in Italy. In: Christensen, Pia \& James, Allison (2008): Research with Children: Perspectives and Practice. Second Edition. New York; Oxford: Routledge, pp 239-259.

Davis, James (2010): Introduction: Emotions in the Field: The Psychology and Anthropology of Fieldwork. In: Davis, James \& Spencer, Dimitrina (Eds.): Emotions in the Field: The Psychology and Anthropology of Fieldwork. California: Stanford University Press, pp. 1-34.

Denzin, Norman \& Lincoln, Yvonne (2011) (Eds.): The SAGE handbook of qualitative research. Fourth Edition. London: Sage Publications.

Edmond, Ruth (2005): Ethnographic research methods with children and young people. In Greene, Sheila \& Hogan, Diane (Eds.): Researching Children's Experience: Approaches and Methods. London: Sage pp 123-140.

Ellis, Carolyn (1991): Emotional Sociology. In: Studies in Symbolic Interaction 12, pp. 123-145.

England, Paula \& Farkas, George (1986): Households, employment, and gender. New York: Aldine.

England, Paula \& Folbre, Nancy (1999): The cost of caring. In: The ANNALS of the American Academy of Political and Social Science 561, 1, pp. 39 - 51.

Erickson, Rebecca \& Stacey, Clare (2013): Attending to mind and body: Engaging the complexity of emotion practice among caring professionals. In: Grandey, Alicia. A., Diefendorff, James. M. \& Rupp, Deborah. E. (Eds.): Organization and management. Emotional labor in the 21st century: Diverse perspectives on emotion regulation at work. New York: Routledge, pp. 175-196.

Fine, Gary \& Sandstrom, Kent (1988): Knowing Children: Participant Observation with Minors. Newbury Park: Sage.

Gansen, Heidi (2017): Researcher Positionality in Participant Observation with Preschool Age Children: Challenges and Strategies for Establishing Rapport with Teachers and Children Simultaneously. In: Castro, Ingrid E., Swauger, Melissa \& Harger, Brent (Eds.): Researching Children and Youth: Methodological Issues, Strategies, and Innovations. Sociological Studies of Children and Youth 22, Emerald Publishing Limited (eBook publication), pp. 81 - 102.

Goosen, Simone, Stronks, Karien \& Kunst, Anton (2014): Frequent relocations between asylum-seeker centres are associated with mental distress in asylum-seeking children: a longitudinal medical record study. In: International Journal of Epidemiology 43, 1, pp. 94-104.

Guillemin, Marilys \& Gillam, Lynn (2004): Ethics, reflexivity, and "ethically important moments" in research. In: Qualitative Inquiry 10, 2, pp. $261-280$.

Howell, Kerry (2013): An introduction to the philosophy of methodology. London: Sage.

Iverson, Roberta (2009): 'Getting Out' in Ethnography - A Seldom-told Story. In: Qualitative Social Work 8, 1, pp. 9-26. 
Kaukko, Mervi, Dunwoodie, Karen \& Riggs, Elisha (2017): Rethinking the ethical and methodological dimensions of research with refugee children. In: Journal of International Educational Research and Development Education (Zeitschrift für internationale Bildungsforschung und Entwicklungspädagogik) 40, 1, pp. 16-21.

Kellett, Mary (2010): Rethinking children and research: Attitudes in contemporary society. Continuum International Publishing Group London: New York.

Knapp, Mark, Hall, Judith \& Horgan, Terrence (2014): Nonverbal Communication in Human Interaction. Eighth Edition. Boston: Wadsworth.

Lewek, Mirjam \& Naber, Adam (2017): Kindheit im Wartezustand. Studie zur Situation von Kindern und Jugendlichen in Flüchtlingsunterkünften in Deutschland. Köln: UNICEF.

Luna, Florencia (2009): Elucidating the Concept of Vulnerability: Layers Not Labels. In: International Journal of Feminist Approaches to Bioethics 2, 1, pp. 121139.

Mandell, Nancy (1991): The least adult role in studying children. In: Waksler, Frances (Ed.) Studying the Social Worlds of Children: Sociological Readings. London: Falmer Press, pp. 38-59.

Marsh, Jackie, Brooks, Greg, Hughes, Jane, Ritchie, Louise, Roberts, Samuel \& Wright, Katy (2005): Digital beginnings: Young children's use of popular culture, media and new technologies. Literacy Research Centre: University of Sheffield.

O'Reilly, Michelle, Dogra, Nisha \& Ronzoni, Pablo Daniel (2013): Research with Children: Theory and Practice. London: Sage.

Parker, Jeffrey, Rubin, Kenneth, Erath, Stephen, Wojslawowicz, Julie \& Buskirk, Allison (2006): Peer relationships, child development, and adjustment: A developmental psychopathology perspective. In: Cicchetti, Dante \& Cohen, Donald J. (Eds.): Developmental Psychopathology, Theory and Method. New Jersey: John Wiley \& Sons, pp. 419-493.

Pearlin, Leonard \& Bierman, Alex (2013): Current issues and future direction in research into the stress process. In: Aneshensel, Carol, Phelan, Jo \& Bierman, Alex (Eds.): Handbook of the sociology of mental health, Second Edition. New York, NY: Springer, pp. 325-340.

Punch, Samantha (2002): Research with Children The same or different from research with adults? In: Childhood 9, 3, pp. 321-341.

Punch, Samantha (2012): Hidden struggles of fieldwork: Exploring the role and use of field diaries. In: Emotion, Space and Society 5, pp. 86-93.

Scheper-Hughes, Nancy (1992): Death Without Weeping. The Violence of Everyday Life in Brazil. California: University of California Press.

Stebbins, Robert (1991): Do we ever leave the field? Notes on secondary fieldwork involvements. In: Shaffir, William \& Stebbins, Robert (Eds.): Experiencing Fieldwork: An Inside View of Qualitative Research. Newbury Park, CA: Sage, pp. 248-255. 
Taylor, Steven (1991): Leaving the field: research, relationships and responsibilities. In: Shaffir, William \& Stebbins, Robert (Eds.): Experiencing Fieldwork: An Inside View of Qualitative Research. Newbury Park, CA: Sage pp. 238-247.

Wagner, Evelyn (2016): Kindesschutz "learning by doing" Das Menschenrecht auf Schutz vor Gewalt geflüchteter Kinder in Sammelunterkünften in Deutschland. M.A. Thesis (unpublished). Berlin: Freie Universität/ Fachbereich Erziehungswissenschaft und Psychologie.

Wendel, Kay (2014): Unterbringung von Flüchtlingen in Deutschland. Regelungen und Praxis der Bundesländer im Vergleich. Frankfurt am Main, Germany: PRO ASYL e. V. 



\title{
Zentrale Erkenntnisse und Empfehlungen
}

\author{
Anne Wihstutz
}

Trotz anderslautender EU-Rechtslage werden geflüchtete Kinder mit ihren Eltern in nicht geeigneten Erstaufnahmeeinrichtungen, Not- und Gemeinschaftsunterkünften untergebracht. Dies geschieht, obwohl auch für diese Kinder die UN-Kinderrechtskonvention gilt, zumal nachdem Deutschland seinen ,,ausländerrechtlichen Vorbehalt“ 2010 zurückgenommen hat. Die vorangestellten Beiträge analysieren und diskutieren auf der Grundlage einer mehrere Monate umfassenden ethnografischen Studie, wie junge, noch nicht schulpflichtige Kinder sich in diesen Verhältnissen behaupten (können). Der Kontext der Studie ist gerahmt von kinderrechtlichen Bestimmungen auf nationaler und internationaler Ebene und geltendem Asyl- und Aufenthaltsrecht in Deutschland in den Jahren 2015 bis 2017.

Im Folgenden werden die wichtigsten Erkenntnisse aus den einzelnen Beiträgen zusammengefasst und in einem flüchtlingsdiskurstheoretischen Zusammenhang eingebettet diskutiert. In einem als Positionspapier betitelten Abschluss werden Handlungsempfehlungen an Politik, Praxis und Forschung formuliert. Ein Nachtrag zur aktuellen politischen Entwicklung seit Beendigung des Forschungsprojekts im Jahr 2017 schließt das Buch.

\section{Zusammenfassung}

Konzipiert als rechtlicher Rahmen für die nachfolgenden empirischen Beiträge untersucht Wihstutz in ihrem Beitrag für die Zeit von 2015 bis 2017 den Stellenwert der Kinderrechte im Verhältnis zum Asyl- und Migrationsrecht für geflüchtete Kinder in Deutschland. In Auseinandersetzung mit dem Hegemoniebegriff von Laclau und Mouffe (1985) zieht sie das Fazit, dass Kinderrechte in Deutschland nicht in diesem Sinne als ,universell“ gelten können, da die Kinderrechte geflüchteter Kinder als ,spezielle Rechte“ nationalstaatlichen migrations- und asylpolitischen Interessen untergeordnet werden. Trotz der Anerkennung der eigenständigen Rechtsträgerschaft von Kindern durch das Bundesverfassungsgericht wirken sich diese kaum in der Rechtsprechung und Rechtswirklichkeit insbesondere für geflüchtete Kinder aus. So werden zentrale Rechte wie der Maßstab des Kindeswohls (oder ,the best interests of the 
child“, wie es im Englischen heißt), das Verbot der Diskriminierung, das Recht auf Entwicklung und das Recht auf Schutz vor Gewalt nach wie vor nicht vorrangig bei allen Maßnahmen, die die Kinder betreffen, berücksichtigt (Artikel 3 Satz 1 UNKRK), wie Wihstutz anhand ausgewählter Bestimmungen zu Unterbringung, Versorgung, zum Schutz der Familie und Zugang zu frühkindlichen Bildungsangeboten für geflüchtete Kinder aufzeigt. Dies widerspricht auch der EU-Richtlinie 2013/33, die auf die besonderen Erfordernisse in der Unterbringung von geflüchteten Kindern abstellt. Diese widersprüchliche Rechtslage wird von Wihstutz als Ausdruck konfligierender Diskurse im Wettstreit um die hegemoniale Deutungsmacht der politischen Identität geflüchteter Kinder analysiert. Es gelte, nicht nur die Mehrdeutigkeiten und Verstrickungen zwischen den Diskursen aufzuzeigen, sondern diese auch als antagonistisch und aufeinander angewiesen zu begreifen. Wihstutz plädiert für eine Öffnung der Diskurse über die gemeinsamen grundsätzlichen Werte, die nicht nur geflüchtete Kinder, sondern das Zusammenleben in der Gesellschaft insgesamt betreffen.

Schulz-Algie bearbeitet in ihrem Beitrag das Thema der Gewalt und den Schutz von Kindern vor Gewalt in den Unterkünften. Ihr Resümee fällt eindeutig aus: Geflüchtete Eltern und ihre Kinder finden in Erstaufnahmezentren und Gemeinschaftsunterkünften in Deutschland nicht die notwendigen Bedingungen, um ein gewaltfreies Leben zu führen. In Auseinandersetzung mit Gewaltdefinitionen von Galtung (1969 und 1990), der WHO (2002 und 2003) und kindheitssoziologischen Ansätzen arbeitet sie aus dem empirischen Datenmaterial die Bedeutung verschiedener Dimensionen von Gewalt in diesen spezifischen Kontexten heraus. Daran anknüpfend entwickelt sie kontextbezogene Thesen zum Kinderschutz. Kinder und ihre Eltern erleben in der spezifischen Unterbringungsform strukturelle Gewalt, die als indirekte Gewalt in gesellschaftlichen Strukturen verankert ist und sich aufgrund asymmetrischer Machtverhältnisse in ungleichen Lebenschancen äußert (vgl. Galtung 1969). Gewalt wird als eine Erfahrung dargestellt, die auf allen Ebenen des Ö̉kologischen Modells (nach WHO 2002) eingelagert ist und diese wechselseitig durchdringt: auf der Ebene des Individuums, auf der Ebene des Mikrosystems in den Beziehungen der Kinder mit ihren Eltern, mit anderen Bewohner*innen und Mitarbeiter*innen sowie in den Beziehungen zwischen Mitarbeiter*innen und Bewohner*innen und schließlich auf der Ebene des Makrosystems, auf der politisch-asylrechtlichen Ebene wie zum Beispiel in Form von Abschiebungen. Fundamentale Rechte von Kindern werden verletzt: Soziale Exklusion, Ressourcenknappheit, mangelnde Privatsphäre, mangelnde Ruhe und Rückzugsmöglichkeiten, unhygienische Zustände der sanitären Anlagen und unzureichende Bedingungen der Essensversorgung in den Unterkünften stellen strukturelle Formen der staatlich verantworteten Gewaltausübung gegenüber geflüchteten Kindern und Eltern dar. Die vielfältigen Formen struktureller Gewalt gegenüber geflüchteten Kindern beschreibt sie als einen „Nährboden“ für 
weitere Gewalterfahrungen von Kindern. Schulz-Algie führt aus, dass und wie prekäre Arbeitsbedingungen des Personals nicht dazu beitragen, kinderschutzrelevante Aufgaben ausreichend wahrzunehmen, allein weil es an qualifizierten Mitarbeiter*innen mangelt. Engagierte und respektvoll den Kindern und Eltern zugewandte Mitarbeiter*innen in den Unterkünften werden indes als Schutzfaktor in der Verhinderung von Gewalt an Kindern identifiziert. Die emotional-positive Bedeutung der Eltern für die Kinder beschreibt sie als einen zentralen Schutzfaktor in der Entwicklung von Resilienzen. Nicht zuletzt sind es die jungen Kinder selbst, die über Ressourcen verfügen bzw. diese sich aneignen, um sich vor den verschiedenen Dimensionen der Gewalt in den Unterkünften zu schützen. Schulz-Algie diskutiert junge Kinder als kompetente Akteur*innen im Schutz gegen Gewalt am Beispiel einer Mobilisierung der Kinder gegen einen Mitarbeiter einer Unterkunft und am Beispiel eines dreijährigen Mädchens, das sich über eigene Strategien mehr Handlungsraum verschafft. Deutlich stellt sie anhand dieser Beispiele heraus, wie Kinder trotz oder gerade aufgrund der diskriminierenden Strukturen in den Unterkünften auf die Unterstützung anderer Kinder sowie auf die respektvolle Wahrnehmung ihrer Anliegen durch Erwachsene angewiesen sind.

Eine akteurszentrierte Perspektive nehmen Fichtner und Trần sowie Scott und Le ein. Die Strategien und Taktiken der Kinder im Umgang mit ihrer Unterbringungssituation stehen in beiden Beiträgen im Zentrum der Analyse. Fichtner und Trần diskutieren Raumerfahrungen, Aneignungsprozesse und Gestaltungspraxen von Kindern in den Dimensionen von physisch-materiellem Raum, Beziehungsraum und Handlungsraum. Sie ordnen die alltäglichen Praktiken der Kinder ein in den Kontext ,großer“" geopolitischer Strategien und migrationspolitischer Machtverhältnisse. In Anbetracht der umfassenden strukturellen Diskriminierung geflüchteter Kinder heben sie die Handlungsfähigkeit von Kindern als relational bedingt hervor. Damit fordern sie gleichermaßen „Opferdiskurse“ und defizitär-homogenisierende Narrative über geflüchtete Kinder heraus. Über „dichte Beschreibungen“ arbeiten sie die Akteur*innenschaft von Kindern in drei Fallstudien heraus: Die Kinder werden beschrieben als sich nicht nur mit diesen Verhältnissen arrangierend, sondern als aktive auf die Umsetzung ihrer Interessen und Bedürfnisse in diesen von Ungewissheiten, Einschränkungen und Unzulänglichkeiten markierten Raum Hinwirkende. Als „doing relations“ bzw. „doing space“ beschreiben Fichtner und Trần das Handeln der Kinder, indem sie Beziehungen und Bewegungsräume verteidigen und gestalten sowie Raum und Zeit für Spiel und Rückzug einfordern und sich Ordnung und Routinen schaffen gegenüber den restriktiven Lebensverhältnissen in den Unterkünften. Dabei werden räumliche und soziale Grenzen auch überschritten, verhandelt und Ordnungen und Verhältnisse in ihrem Status quo irritiert. Aus ihrer Analyse entwickeln sie konkrete Handlungsempfehlungen an Politik und zivilgesellschaftliche Akteur*innen. 
Das tägliche Leben von Kindern in einer ausgewählten Unterkunft und ihr in der UN-Kinderrechtskonvention verankertes Recht auf Spiel stehen im Fokus von Scott und Le. Sie analysieren, wie die drei in Beziehung stehenden Aspekte der sozialen Produktion von Raum den Alltag von Kindern und ihre Spielerfahrungen formen. Sie machen deutlich, dass „Raum“ sozial hervorgebracht wird (Lefebvre 1991). Kinder sind an dieser Hervorbringung von Raum beteiligt, indem sie diesen nach ihren eigenen Interessen aushandeln und nutzen, auch wenn sie damit unter Umständen gegen die etablierten Regeln der Unterkunft verstoßen. Scott und Le identifizieren Handlungspraxen der Kinder als Strategien und Taktiken im Umgang mit den durch Mangel und Intransparenz gekennzeichneten alltäglichen Lebensbedingungen in den Unterkünften. Dabei führen Kinder Konflikte als eine Strategie bewusst herbei, um mit wahrgenommenen Bedrohungen aktiv umzugehen. In ihrem Verhalten zeigt sich demnach, wie die Kinder ihre soziale Umwelt interpretieren und sich selbst darin als verwundbar erleben. Damit stellen die Verhältnisse selbst einen Risikofaktor für die Entwicklung und das Wohlsein geflüchteter Kinder dar. Ein angst- und konfliktfreies Spielen ist den Kindern in den Unterkünften kaum möglich. Das Recht auf Spiel ist in diesen Verhältnissen schwer umsetzbar.

Die Beiträge von Scott und Trần arbeiten schließlich die besonderen Herausforderungen der Forschung mit Kindern in extrem prekär und ungesicherten Verhältnissen heraus. Im Zentrum von Scotts Beitrag stehen Fragen von Beziehungsaufbau und -beendigung im Kontext ihrer Unterbringung in Unterkünften für Geflüchtete. Die Gestaltung und Qualität von Feldbeziehungen ist in der interpretativen, konstruktivistisch verstandenen ethnografischen Forschung zentral für die Entwicklung „dichter Beschreibungen“ vom Forschungsfeld und seinen sozialen Prozessen (Denzin \& Linclon 2011; Howell 2013). Über Scotts nuancierte Beschreibung darüber, wie ethnografische Fakten und Beziehungen im alltäglichen Miteinander zwischen den Forschungsteilnehmer*innen aufgebaut werden, lässt sie die Leser*innen daran teilhaben, wie die Ethnografin ihr (Forschungs-)Wissen entwickelt und zu ihren Schlussfolgerungen gelangt. Scott verweist auf die besondere Bedeutung der Selbstreflexion über die eigene Positionalität als Erwachsene gegenüber Kindern sowie auf die Bedeutung von Zuschreibungen als „Ausländerin“ bzw. als Mitglied einer ethnischen Minderheit für die eigene Sensibilisierung der Unterschiede unter den Kindern und ihrer je individuellen biografischen Erfahrungen. Sie beschreibt die Auseinandersetzung als ,reflexive practice“, die zu einer differenzierten Beziehung zu jedem der beteiligten Kinder führte. In Auseinandersetzung mit den durch mangelnde Ressourcen gekennzeichneten Lebensverhältnissen der Kinder in den Unterkünften weist Scott auf die Bedeutung der Beziehungen zu den Forscherinnen als soziales Kapital und als praktische, konkrete Unterstützung der Eltern hin. Aus der asymmetrischen Beziehungskonstellation erwächst der explizit formulierte Anspruch, sich bereits während 
des Forschungsprozesses für die Rechte und Interessen der Forschungsteilnehmer*innen einzusetzen bzw. unmittelbar von Nutzen für sie zu sein (O'Reilly 2013: 49). Dilemmata, die auf diesen Anspruch verweisen, beschreibt Scott als teilweise nicht lösbar und damit als besonders herausfordernd. Nach Scott tangiert ethnografische Forschung die emotionale, analytische und politische Ebene (Davis 2010). Die unterschiedlichen Ebenen sind von der Ethnografin in der eigenen Wahrnehmung zu differenzieren, um die eigenen emotionalen Verstrickungen und ethischen Dilemmata erkennen zu können.

Auch Trần stellt in ihrem Beitrag die eigenen Verstrickungen von Forscher*innen als bedeutsame Ressource für den Erkenntnisgewinn in der ethnografischen Forschung heraus. Das Bewusstsein für die verschiedenen Machtverhältnisse, in die die Forschung mit Geflüchteten und insbesondere die Forschung mit geflüchteten Kindern eingelassen ist, wird von ihr als Erfordernis für eine ethisch fundierte, reflektierte Forschungspraxis formuliert. In Auseinandersetzung mit den konkreten empirischen Erfahrungen in Unterkünften für Geflüchtete beschreibt sie vielfältige Fallstricke, die einer Forschung aus ethischer Perspektive begegnen können, im Besonderen wenn diese anstrebt, Forschungsteilnehmer*innen nicht nur als Datenquelle wahrzunehmen, sondern auch für diese von konkretem Nutzen zu sein. Als „engagierte Forschung" benennt Trần eine Forschungspraxis, die im Feld Position bezieht, andere Formen der Veröffentlichung von Forschungsergebnissen wählt und zu konkreten Veränderungen im Interesse der Forschungsteilnehmer*innen beizutragen anstrebt, über Handlungsempfehlungen für Praxis und Politik. Ohne die damit verknüpften Herausforderungen und Dilemmata für die Forscher*innen zu vernachlässigen, plädiert Trần in ihrem Beitrag für eine machtkritische, selbstreflexive Forschung, die sich ihrer Verstrickungen in machtvolle Verhältnisse bewusst ist und zum Abbau sozialer Ungleichheit beitragen will. In diesem Sinne versteht sie sozialwissenschaftliche Forschung als politisch.

„The exceptional crisis faced by refugee children renders their state of suffering an inalienable facet of their lives, yet, they show agency and some form of power to control their fate in the manner in which they engage, negotiate the condition of subalternity“ (Sen 2018: 109).

Die Geschichte der Unterdrückten (,Subalternen“) ist nie nur eine Erzählung ihrer Unterdrückung. In den vorangestellten Beiträgen wurden Kinder nicht als den Verhältnissen nur hilflos Ausgelieferte vorgestellt. Vielmehr werden Kinder als handelnde Akteur*innen ,dicht beschrieben“, ohne ihre gesellschaftliche Marginalisierung, ihre Vulnerabilität und Diskriminierungserfahrungen in Abrede zu stellen.

Über die teilnehmende Beobachtungen am Leben der Kinder in den Unterkünften konnte dargelegt werden, wie Kinder versuchen, mit Verhältnissen umzugehen, die als gewaltvoll und diskriminierend identifiziert wurden, und wie sie versuchen, sich darin zu bewegen und zu behaupten. Wie sich Normalität in ihr Leben im „Wartezustand“ (Lewek \& Naber 2017), während des 
Asylverfahrens übersetzt. Es sind die kleinen Episoden, wie wir sie in den einzelnen Beiträgen beschrieben haben, in den ,minute processes of the everyday" (Sen 2018: 108), in denen sich das Leiden der Kinder, aber auch ihre Handlungsfähigkeit zeigen. Die ethnografische Herangehensweise ermöglichte den Zugang zu diesen Prozessen, Situationen, Aktivitäten. Darüber konnten die unterschiedlichen Strategien der Kinder und die sie hemmenden oder unterstützenden Einflüsse im Umgang mit konkreten Verhältnissen vor Ort herausgearbeitet werden. Ihre Geschichte bzw. ihre Geschichten, wie sie hier zum Ausdruck gebracht worden sind, können nicht auf ein Narrativ des Leidens und der Passivität reduziert werden. In den spezifischen Beziehungen, die sich während des Forschungsprozesses entwickelten und beobachtet werden konnten, wurden die Kinder als handelnde, aktive Subjekte wahrgenommen, die sich individuell und kollektiv artikulierten. Selbstreflektierend wurde wiederholt die Frage gestellt, welche und wessen Perspektiven in der Forschung zum Ausdruck gebracht werden, um im Feld und in der Forschungsbeziehung eingelagerte Machtkonstellationen aufzudecken und kritisch zu analysieren. Diese selbstreflexive, machtkritische Analyse begleitete das Vorhaben von Anfang an, insbesondere da die Studie von dem Anspruch getragen wurde, unmittelbar von Nutzen für die Forschungsteilnehmer*innen zu sein.

\section{Fazit und Ausblick: Gegen die doppelte Viktimisierung von geflüchteten Kindern}

Die Fallanalysen und dichten Beschreibungen von Kindern in den Unterkünften fordern auf zum differenzierten Hinschauen und zum aufmerksamen Hinhören, wenn Kinder und Familien sowie Mitarbeiter*innen vom Alltag in Unterkünften erzählen. Beeinflusst von der öffentlichen Debatte sind die eigenen Beobachtungen, Interpretationen sowie auch die Äußerungen von Geflüchteten und Nichtgeflüchteten kritisch zu untersuchen: Welche Bilder und Narrative dominieren, wer wird wann gefragt und wem wird zugehört? Was gilt als legitimes bzw. legitimiertes Wissen und Sprechen? Welche Handlungsräume verschaffen sich diejenigen, die im dominanten Repräsentationsregime in Europa als „Andere“, als „Außenstehende“ als „Flüchtlinge“ positioniert werden?

Im Folgenden wird abschließend das Verhältnis der Mehrheitsgesellschaft bzw. der Aufnahmegesellschaft zu Geflüchteten aus einer politisch strukturellen Perspektive beleuchtet. In einer postkolonialen Reanalyse des Flüchtlingsbegriffs stellen Niedrig und Seukwa (2010) die identitätsstiftende Funktion bzw. Funktionalität des rechtlich-politischen und sozialen Konstrukts „Flücht- 
ling“ für die national kodierte Selbstdeutung der Mehrheitsgesellschaft heraus. ${ }^{1}$

Erst durch die Inbeziehungsetzung der Kategorie Flüchtling mit der national kodierten Selbstdeutung der Aufnahmegesellschaft, in der Figur einer Triade von Opfer - Täter - Retter werde es möglich, den Flüchtlingsbegriff in seiner diskursiven Funktionsweise zu dekonstruieren (vgl. Niedrig \& Seukwa 2010). Komplexe rechtliche Auslegungen legitimer oder illegitimer Fluchtgründe werden im Alltagsverständnis dichotom gegenübergestellt: „,echte Flüchtlinge“ versus „Scheinasylanten“ (vgl. Holmes \& Castañeda 2016). „Ein ,echter Flüchtling' wird als ,hilfloses, unschuldiges Opfer" imaginiert". Gendertheoretisch analysiert, ist das Opfer ,weiblich konnotiert: passiv, hilflos, anspruchslos/bescheiden, leidensfähig, zu Anpassung und Unterordnung bereit“ (Niedrig \& Seukwa 2010: 185). ${ }^{2}$ Aus einer ,paternalistischen Mitleidshaltung“" (ebd.: 192) heraus werden Erwachsene infantilisiert bzw. als unfähig beschrieben, um ihrer (Erziehungs-)Verantwortung ihren Kindern gegenüber gerecht zu werden (vgl. Beitrag von Schulz-Algie). Pupavac nennt es die „Viktimisierung“ von Geflüchteten (vgl. Pupavac 2008). Flüchtlinge werden zunehmend als traumatisiert, als Opfer von Kriegen, Naturkatastrophen und Konflikten dargestellt. ${ }^{3}$ Traumatisierte Menschen sind krank, benötigen Hilfe und sind zu betreuen. So richtig und wichtig die Hilfe für traumatisierte Menschen auch ist und gefordert werden muss, so folgenreich ist der Opferdiskurs in Bezug auf die Rechte von Schutzsuchenden. Statt ihre Handlungs- und Selbstentscheidungsfähigkeit zu stärken, fokussiert der Krankheitsdiskurs auf die Schwächen und verweist auf die Hilfebedürftigkeit Geflüchteter von Expert*innen (ebd.). Die Abhängigkeit Geflüchteter, insbesondere Traumatisierter, wird hervorgehoben. „In short, permissive empathy develops professional management of refugees' welfare, rather than necessarily greater refugee rights" (Pupavac 2008: 280). ${ }^{4}$

1 Als Diskursanalyse angelegt, dekonstruieren Niedrig \& Seukwa (2010) die essentialisierende Kategorie Flüchtling und machen sichtbar, dass es sich hierbei um ein politisch-rechtliches und soziales Konstrukt handelt (vgl. Niedrig \& Seukwa 2010).

2 Dieser Opferfigur gegenüber stünde die Figur vom Flüchtling als Täter. In der Triade Opfer - Täter - Retter wird diese Figur beschrieben als fordernd, durchsetzungsstark, dynamisch und aktiv. Geflüchtete mit diesem Auftreten wird unterstellt, keine ,richtigen Flüchtlinge“ zu sein und den staatlichen Schutz für ihre Zwecke zu missbrauchen.

3 Während des Kalten Krieges galten Flüchtlinge vorrangig als politisch Verfolgte, Dissidenten und Widerstandkämpfer, die im Wettkampf der Systeme gerne zur Darstellung der eigenen Systemüberlegenheit instrumentalisiert wurden. Erst in den 1980er Jahren wurden Flüchtlinge verstärkt unter gesundheitlichen Aspekten, als traumatisiert betrachtet, nachdem die American Psychiatric Association Posttraumatische Belastungsstörungen als Krankheit anerkannt hatte (Pupavac 2008: 278).

4 Pupavac bezieht sich hierbei auf den von Parsons geprägten Begriff permissiver Empathie, der die Abhängigkeit eines Kranken von einer gesunden Person bzw. von Expert*innen beschreibt: Die als krank beschriebene Person gibt ihre Selbstständigkeit auf und überträgt die Verantwortung für ihr Leben in die Hand von Expert*innen. Damit 
Die Hilfebedürftigkeit und die Adressierung als Opfer erfährt in Bezug auf Kinder eine diskursive Dopplung: Als sehr junge Kinder sind sie den Verhältnissen ausgeliefert, zumal sie weder entwicklungsbedingt noch rechtlich, politisch, wirtschaftlich oder kulturell als gleichberechtigte Akteur*innen konzeptualisiert werden, die sich den Verhältnissen von Flucht und Vertreibung, Rassismus und Gewalt aktiv widersetzen könnten. Kinder werden häufig als traumatisiert und verwundbar beschrieben (vgl. Kaukko \& Wernesjö 2016). Damit werden Kinder auf Stereotype reduziert, die ihrer Verschiedenheit im Umgang mit den Verhältnissen und ihren vielschichtigen Erfahrungen nicht gerecht werden. Auch entscheiden häufig Erwachsene über das, was für Kinder das Beste sei (vgl. Boyden zitiert nach Kaukko \& Wernesjo 2016: 3). Auch im Ankunftsland sind sie nicht sicher und weiteren Stressoren ausgesetzt, die ihr Wohlsein nachträglich gefährden können (vgl. Maragel \& Manachi 2018).

Wie in den vorangestellten Beiträgen deutlich geworden ist, werden elementare Rechte von Kindern in den Unterkünften missachtet. Kinder sind darüber hinaus mitbetroffen, wenn ihre Eltern unter den Bedingungen des Asyls psychisch und physisch leiden. Insofern verstetigt die asylrechtliche Lage den Opfer - wenn nicht sogar den Objektstatus geflüchteter Kinder. Es sind diese Verhältnisse, die insbesondere die Vulnerabilität geflüchteter Kinder mithervorbringen (vgl. Kaukko et al. 2017: 17).

Kinder verfügen unter bestimmten Umständen auch in solch widrigen Umständen über die Fähigkeit, ihre Interessen zu verteidigen bzw. Strategien zu entwickeln, um sich zu schützen. Ihre Agency muss nicht im Widerspruch zu ihrer Schutzbedürftigkeit und Vulnerabilität stehen. Wie wir es anhand konkreter Situationen beschrieben haben, können Kinder sowohl handlungsfähig als auch schutzbedürftig sein (siehe die Beiträge von Fichtner \& Trần, von Scott \& Le und von Scott; vgl. Kaukko \& Wernesjö 2016).

Die Kritik am Traumatisierungs- und Viktimisierungdiskurs wendet sich gegen die damit einhergehende Individualisierung und Depolitisierung von Flucht und Migration (Pupavac 2008). ${ }^{5}$

Weniger im Fokus der aktuellen öffentlichen Diskurse um Flucht und Migration stehen die politischen Dimensionen von Vertreibung, Elend und Gewalt oder die historische Perspektive, um die globalen Zusammenhänge verstehen und Verantwortlichkeiten benennen zu können. ${ }^{6}$ Derart ist es möglich, dass

würden die Kranken aus jeder Verantwortung für ihr Handeln entlassen (vgl. Pupavac 2008).

5 Siehe auch die Kritik von Pupavac 2001 an der UN-Kinderrechtskonvention. Sie kritisiert die Orientierung am Individuum, die den Einfluss komplexer sozioökonomischer, politischer und kultureller Zusammenhänge auf die Ausgestaltung von Kindheit vernachlässige (vgl. Pupavac 2001).

6 Flucht und Migrationsbewegungen bringen einerseits immer neue Grenzziehungen hervor, welche in territorialer, ethnischer, religiöser, kultureller, sozialer, ökonomischer oder sprachlicher Art regulierend, kontrollierend und mitunter gewaltsam einschränkend wirken. Zugleich problematisieren und verändern Migrationsbewegungen 
sich ein Aufnahmeland wie Deutschland als „Retter“" bzw. „Opfer“ inszenieren kann (s. o., Triademodell nach Niedrig \& Seukwa 2010):

„,[I]n der Dreiecksbeziehung, Täter-Opfer-Retter' kommt ,Deutschland' entweder die Position des ,Retters' (der ,wirklich Verfolgten') oder des ,Opfers' (von ,Asylmissbrauch') zu, unter keinen Umständen jedoch die Position des ,Täters““” (Niedrig \& Seukwa 2010: 185,).

Aus berufsethischer Perspektive gäbe es hinlängliche Gründe, um gegen die tagtäglichen Rechtsverletzungen an Kindern in den Unterkünften zu protestieren (vgl. Initiative ASH 2016). Initiativen wie die Entwicklung von Mindeststandards in Unterkünften für Geflüchtete (vgl. BMFSJF 2017), die Bereitstellung von Sprungbrettangeboten für Familien mit jungen Kindern (Masterplan Integration und Sicherheit, Berlin 2016) oder ein Unterbringungs-TÜV zur Messung von Kinderrechten in den Unterkünften (Save the Children Deutschland 2018) zielen darauf ab, die Verhältnisse der Menschen in diesen Unterbringungsformen konkret zu verbessern. Kritiker*innen befürchten, dass solche Ansätze von den eigentlichen Problemen ablenken, wie von der strukturellen Diskriminierung in der Unterbringung, der Versorgung und der Teilhabe von Geflüchteten (vgl. Pupavac 2008). Dazu kommt die Unsicherheit des Aufenthaltsstatus, die Angst vor Abschiebungen, das Leiden unter der Trennung von Familienangehörigen und die Sorgen um Verwandte und Freund*innen in der Heimat. Die Verhältnisse, die es überhaupt möglich machen, dass Menschen derart fremdbestimmt, unter Missachtung ihrer Rechte verwaltet werden, stehen nicht mehr im Fokus der Kritik. Damit erlebt die Flüchtlingsarbeit von Aktivist*innen und Menschenrechtsakteur*innen eine Depolitisierung (ebd.). So fassen Niedrig und Seukwa zusammen:

,[N]ur in dem Maße, in dem die (post-)kolonialen Zusammenhänge ,vergessen“, verleugnet, ausgeblendet werden“, könne sich „Deutschland als ,Retter“ oder ,Opfer" in der national-definierten Selbstwahrnehmung positionieren“ (Niedrig \& Seukwa 2010: 187).

Kein Staat ist rechtlich dazu verpflichtet, Schutzsuchende aufzunehmen. In der Genfer Flüchtlingskonvention von 1951 wurde explizit nicht die staatliche Verpflichtung zur Aufnahme Schutzsuchender eingefügt, sondern ausschließlich das Recht des Individuums, Asyl zu beantragen. Die Ungewissheit darüber, wann der Staat die Entscheidung treffen und wie sie ausfallen wird,

und -diskurse diese Grenzziehungen. Migrationsphänomene und Grenzziehungen bringen sich also wechselseitig hervor und sind in dieser wechselseitigen Gebundenheit zu untersuchen. Dabei ist die Annahme grundlegend, dass die zur Rede stehenden Grenzen - beispielsweise auch die politisch verordneten territorialen - nicht einfach „da“ sind, sondern auf verschiedenen gesellschaftlichen Ebenen durch individuelle und kollektive Akteur*innen diskursiv und performativ thematisiert, dadurch kontinuierlich neu und anders hervorgebracht, umkämpft, neu gezogen, umdefiniert werden und stets materiell erfahrbar sind. 
produziert Abhängigkeit und Unselbstständigkeit bei Geflüchteten. Umso bedeutsamer ist folglich, dass Geflüchtete in die Position versetzt werden, überhaupt Rechte für sich reklamieren zu können, also ,das Recht, Rechte zu haben" (Arendt 2001). Hierauf gilt es, politisch, sozial, kulturell und juristisch in der Aufnahmegesellschaft hinzuwirken. Die Allgemeinen Menschenrechte wie auch die universellen Rechte des Kindes bieten in diesem Bestreben die ethisch-soziale Grundlage, mit der die Möglichkeiten von (geflüchteten) Kindern, ihre Rechte überhaupt in Anspruch nehmen zu können, zu verhandeln sind. Die Wirkmächtigkeit von Kinderrechten und Menschenrechten zeigt sich folglich nicht (nur) in ihrer legalen Umsetzung in nationales Recht, sondern als ethisch-sozialer Begründungsrahmen. Auch in konkreten politischen Programmen, die den Zugang zu Wohnungen und Gesundheitsleistungen und die soziale und wirtschaftliche Beteiligung der Betroffenen ermöglichen, sind Wege der Umsetzung von Menschen- bzw. Kinderrechten. Dazu bedarf es der offenen und öffentlichen Auseinandersetzung über die ethisch-moralischen Forderungen und Ansprüche derer, deren Forderungen bislang nicht gehört oder umgesetzt wurden. , $[\mathrm{H}] \mathrm{uman}$ rights exist whether or not the makers and interpreters of law have had the wisdom and opportunity to reflect these right in actual legislation“"(Sen 2007: 236). Die ethisch-sozialen (und rechtlichen) Ansprüche geflüchteter Kinder sind Gegenstand der Auseinandersetzung auf der politischen Ebene, in den Medien, in der Verwaltung und auf Seiten von Aktivist*innen. Indem Kritik an den Verhältnissen geübt wird und (Kinder-) Rechtsverletzungen öffentlich gemacht werden, bekommen die Menschenrechte eine praktische, konkrete Relevanz.

Als Kindheitswissenschaftlerinnen sehen wir die Aufgabe sozialwissenschaftlicher Forschung darin, mit unseren spezifischen Mitteln, Methoden und Fragen zu einem besseren Verständnis von Kindheit beizutragen. Indem wir den Fokus auf den Alltag von jungen Kindern gelegt haben, konnte die Wirkmächtigkeit ineinandergreifender Ordnungen wie der migrationspolitischen und der generationalen Ordnung herausgearbeitet und in ihrer Bedeutung für das Kinderleben junger geflüchteter Kinder diskutiert werden. Dabei wurden junge Kinder als soziale Akteur*innen nachgezeichnet, die sich auf ihre Art und Weise, mit ihren je spezifischen Fähigkeiten mit den Verhältnissen aktiv und subjektiv auseinandersetzen. Indem die Studie das Ineinandergreifen diskriminierender und gewalttätiger Strukturen mit dem Handeln der Akteur*innen im Feld verzahnt analysiert, können ihre Erkenntnisse dazu beitragen, die Rechte geflüchteter Kinder und ihrer Eltern auf umfassende gesellschaftliche Partizipation, Schutz und Versorgung zu stärken. Über die (selbst-)reflexive Auseinandersetzung mit den eigenen Verstrickungen gesellschaftlicher Privilegien, Rassismen und Vorurteilen in der Forschung leistet die Studie wichtige Erkenntnisse zur Weiterentwicklung machtkritischer forschungsethischer Fragen. 


\section{Positionspapier}

Auf der Grundlage der empirischen Erkenntnisse wurde ein Positionspapier veröffentlicht mit grundsätzlichen Forderungen an Politik und Praxis (vgl. ISTA 2018). Darin heißt es:

Die Unterbringung junger geflüchteter Kinder in Deutschland in Massenunterkünften oder Notunterkünften ist weder förderlich für die kindliche Entwicklung noch im Sinne der Aufnahme in die Mehrheitsgesellschaft. Kinder und ihre Eltern leben in Unsicherheit und Ungewissheit in diesen Unterbringungsformen. Damit einhergehend ist es für sie faktisch unmöglich, ihr Familienleben selbstbestimmt zu führen. Junge geflüchtete Kinder finden in diesen Unterbringungsformen keinen „Raum“, der ihren Interessen und Bedürfnissen gerecht werden kann.

Wir fordern grundsätzlich:

1. die Abschaffung von Sonderstrukturen für Geflüchtete, die zu Ungleichbehandlung in der Unterbringung, der Bildung, der Gesundheitsversorgung und der finanziellen Versorgung geflüchteter Kinder und ihrer Familien führen und ihre gesellschaftliche Teilhabe massiv einschränken,

2. die Anerkennung der besonderen Schutzbedürftigkeit von geflüchteten Kindern und ihren Eltern. Die Bundesregierung hat ihrer Verpflichtung nachzukommen, das Menschenrecht von Kindern auf Schutz vor jeder Form von Gewalt (Artikel 19 UN-KRK), das Recht auf Nichtdiskriminierung (Artikel 2), das Recht auf Berücksichtigung des Wohles des Kindes (Artikel $3 \S 1$ ), das Recht auf Entwicklung (Artikel 6) und das Recht auf Berücksichtigung der Meinung des Kindes (Artikel 12) unverzüglich und umfassend umzusetzen.

3. Wir fordern die Umsetzung des Rechts der Kinder auf Familienzusammenführung. Die Aussetzung des Familiennachzugs für Personen mit subsidiärem Schutz muss umgehend beendet werden. Die Familie steht unter dem besonderen Schutz der staatlichen Ordnung (vgl. Artikel 6 GG, Artikel 8 der Europäischen Menschenrechtskonvention, EMRK, Artikel 24 Abs. 3 der EU Grundrechtecharta).

Wir fordern bezogen auf das Wohnen von Kindern mit ihren Familien:

1. Unabhängig von ihrem Aufenthaltsstatus sind Kinder und ihre Eltern dezentral in Wohnungen unterzubringen bzw. ist ihnen der Zugang zum Wohnungsmarkt zu ermöglichen.

2. Für den Fall, dass die Unterbringung von Kindern und ihren Eltern in eigenen Wohnungen nicht sofort möglich ist, braucht es kindgerechte Wohnverhältnisse, die Rückzugs- und Spielorte bereithalten. Privaträume müssen abschließbar sein. Verbindliche Schutzstandards sind umzusetzen, und ihre Einhaltung ist zu kontrollieren. 
3. Für den Betrieb von Unterkünften für geflüchtete Menschen ist eine Betriebserlaubnis gemäß § 45 SGB VIII einzuführen, wenn weiterhin Kinder und ihre Eltern in diesen Verhältnissen wohnen müssen. Die Unterbringungssituation, die prekäre finanzielle Lage unter dem Grundsicherungsniveau sowie das beengte Zusammenleben mit fremden Personen gefährden und unterminieren die Kompetenzen und Autonomie von Eltern, ihr Kind zu schützen und zu fördern. In der gemeinsamen Unterbringung mit ihren Eltern sind die Ressourcen für gesundheitliche und medizinische Versorgung, für die gesellschaftliche Integration sowie partizipative Strukturen und Beschwerdemöglichkeiten nach $\S 45$ SGB VIII für Kinder sicherzustellen.

4. Die Anerkennung von Kindern als soziale Akteure*innen bedeutet, dass sie an Entscheidungsprozessen beteiligt werden müssen, so auch in Fragen ihrer Unterbringung und in der Wahl des Wohnortes. In den Unterbringungsverhältnissen beinhaltet dies die partizipative Einbeziehung von Kindern in Raumplanung und -gestaltung.

5. Solange es Gemeinschaftsunterkünfte gibt, braucht es ein System von Qualitätsentwicklung und -sicherung, an dem Bewohner*innen, Mitarbeiter*innen und Trägerverbände beteiligt sind. Ziele sind Transparenz der Rollenverhältnisse und Abläufe, die Verbesserung der Arbeitsprozesse und die Entwicklung partizipativer Strukturen.

Wir fordern bezogen auf die Zusammenarbeit mit Kindern und ihren Eltern:

1. Kinder und ihre Familien sind als Individuen mit ihren jeweiligen Kompetenzen und Ressourcen anzuerkennen. Ihre unterschiedlichen Bedürfnisse und Interessen sind jeweils im Einzelfall und stets neu zu ermitteln. Die Kompetenzen der Eltern sowie ihre Verantwortung für ihre Kinder sind zu respektieren.

2. Die Bedarfe geflüchteter Kinder und ihre Eltern nach Leistungen entsprechend $\S \S 23$ ff. SGB VIII sind vom Moment ihres Schutzgesuchs bzw. ihrer Asylantragsstellung zu ermitteln und zu versorgen. Geflüchtete Eltern sind über das Hilfe- und Unterstützungssystem zu informieren und durch Unabhängige zu beraten.

3. Es sind verbindliche Beteiligungs- und Beschwerdemöglichkeiten für die Belange von Kindern und Familien in den Unterkünften zu schaffen und ernst zu nehmen, insbesondere in Bezug auf kinderschutzrelevante Entscheidungen, Aktivitäten und Maßnahmen in den Unterkünften. Räume der Selbstorganisation unter Geflüchteten sind zu fördern und zu ermöglichen.

4. Mitarbeiter*innen müssen qualifiziert sein für die (sozial-)pädagogische Arbeit mit jungen Kindern und über ein fachliches Repertoire verfügen, das ihnen hilft, sensibel mit traumabedingten Bedürfnissen der Kinder umzugehen. Der aktuelle Schlüssel von Kinderbetreuer*innen pro untergebrachter Person umfasst 0,015 Vollzeitstellen. Ein Qualifikations- und Betreuungsstandard, wie er für die frühkindliche Bildung in Kindertagesein- 
richtungen gilt, ist auch in Unterkünften für geflüchtete Menschen mit Kinderbetreuungsangeboten verbindlich einzuführen.

5. Konzeptionell und finanziell ist ein Stellenverhältnis zwischen (sozial-)pädagogischen Mitarbeiter*innen und Bewohner*innen zu gewährleisten, das den umfangreichen Beratungs- und Betreuungsaufgaben von Sozialarbeiter*innen entspricht. Der aktuelle Betreuungsschlüssel für Berlin von einer 0,006 qualifizierten Vollzeitstellen pro untergebrachter Person entspricht nicht den ethischen Standards guter Sozialer Arbeit.

6. Konzeptionell und finanziell abgesichert sind Reflexionsräume für Mitarbeiter*innen zu schaffen, die zur (eigenen) Rollen- und Auftragsklärung beitragen und die es ihnen ermöglichen, normative Annahmen und Stereotype zu hinterfragen und diskriminierende Strukturen, Handlungen und Haltungen zu erkennen und abzubauen.

7. Die Vernetzung im Sozialraum mit allen relevanten Akteuren ist als oberstes Ziel anzusehen, um tragfähige Brücken in die Aufnahmegesellschaft zu bauen.

Wir fordern eine öffentliche Auseinandersetzung in den Medien, auf Bundesund Länderebene sowie im Bildungsbereich darüber, wie die gesellschaftliche Teilhabe von geflüchteten Kindern und ihren Eltern in der Aufnahmegesellschaft gestärkt werden kann. Dabei ist von einem Kinderschutzverständnis auszugehen, welches die Agency von Kindern anerkennt und ein umfassendes und mehrdimensionales Verständnis von Gewalt berücksichtigt. Die öffentliche gesellschaftliche Debatte ist unter maßgeblicher Beteiligung von Geflüchteten jeden Alters, jeder Herkunft, jeder Sprache und jedes Geschlechts, unabhängig von ihren Bleibeaussichten zu führen. Es gibt keine Hierarchie des Leids! Die Menschenrechte der Kinder und ihrer Familien sind universell und nicht verhandelbar.

Gezeichnet von den Mitarbeiterinnen des Forschungsprojekts Berlin, Februar 2018

\subsection{Nachtrag: Aktuelle Entwicklungen}

Seit Beendigung der Forschung und der Vorstellung der Projektergebnisse vor Vertreter*innen aus Politik, Praxis und Wissenschaft am 22. September 2017 in Berlin hat sich der „Flüchtlingsdiskurs“ in Deutschland und Europa zunehmend verschärft. Aus der Bundestagswahl zur 19. Legislaturperiode ging die rechtsnationalkonservative Partei Alternative für Deutschland (AfD) als drittstärkste Fraktion im Deutschen Bundestag hervor. In den nachfolgenden Verhandlungen möglicher Regierungskoalitionen wurde die Frage der zukünftigen 
Ausrichtung der Migrations- und Asylpolitik zur Zerreißprobe. In der Neuauflage der Großen Koalition aus SPD, CDU und CSU formulieren die Regierungsparteien das erklärte Ziel, die Zuwanderung von Schutzsuchenden auf jährlich 180.000 bis 220.000 Personen einzuschränken und schnellere Abschiebungen zu ermöglichen (vgl. Koalitionsvertrag 2018: 104, 109). Die Liste sogenannter sicherer Herkunftsstaaten soll auf Algerien, Marokko und Tunesien sowie auf weitere Staaten mit einer regelmäßigen Anerkennungsquote unter 5 Prozent ausgeweitet werden. Schutzsuchende aus diesen Ländern $(\S 29 \mathrm{a}$ AsylG) sind verpflichtet, bis zum Entscheid über den Asylantrag und im Falle einer Ablehnung bis zur Ausreise bzw. Abschiebung in Erstaufnahmeeinrichtungen zu verbleiben. Das heißt, auch die Kinder von Asylsuchenden verbleiben so lange in diesen Einrichtungen. Die neue-alte Koalitionsregierung hat den Nachzug von Familienangehörigen subsidiär Schutzberechtigter nach einer zweijährigen Aussetzung (s. o.) mit Beginn 1. August 2018 auf maximal 1000 Personen pro Monat begrenzt. Asylverfahren und Abschiebungen sollen bereits überwiegend direkt an den (EU-)Außengrenzen durchgeführt werden. Der Innenminister Horst Seehofer (CSU) plant bundesweit die Etablierung sogenannter zentraler AnKER-Einrichtungen, in denen Ankunft, Entscheidung, kommunale Verteilung bzw. Rückführung (AnKER) konzentriert ablaufen sollen. Der Aufenthalt Schutzsuchender in diesen Zentren ist geknüpft an die Dauer der Bearbeitung ihres Antrags bzw. bei abschlägigem Bescheid gilt die Residenzpflicht bis zur Abschiebung oder „, freiwilligen Rückkehr“ (vgl. Koalitionsvertrag 2018). Bereits heute kann die Residenzpflicht nach geltendem Recht formal bis zu 24 Monate je nach Bundesland umfassen ( $\$ 47$ Abs. 1b Satz 1 AsylG). Eine Verschlechterung der Lebensverhältnisse insbesondere auch von Kindern ist in diesen Einrichtungen zu befürchten: In den als Vorbild für die AnKER-Zentren geltenden Massenunterkünften in Manching und Bamberg ist der Kontakt zur Außenwelt, zur einheimischen Zivilbevölkerung und Expert*innen wie Psycholog*innen und Rechtsberater*innen kaum gegeben (Zeit online, 24.1.2018; Die Zeit Nr. 5 2018, 25.1.2018). Die gesundheitliche Versorgung von Kindern wie auch ihre Ernährungssituation sind alarmierend, der Zugang zu Bildungseinrichtungen ist nicht gegeben (vgl. Hildegard Lagrenne-Stiftung 2016).

Derweil wird auf der Ebene der EU-Mitgliedsstaaten die Einrichtung sogenannter Auffanglager von Migrant*innen außerhalb Europas diskutiert, in die Schutzsuchende abgeschoben werden sollen. Zeitgleich rüstet die Europäische Union weiter in die Absicherung ihrer Außengrenzen auf.

Wie die jüngsten Meldungen des Flüchtlingshilfswerks der Vereinten Nationen zeigen, steigt die Zahl der Flüchtenden und Zwangsvertriebenen weltweit an (vgl. UNHCR 2018). Nur ein Bruchteil von ihnen kommt in Europa an. Statt die Menschenrechte von Schutzsuchenden zu stärken, setzen Europa und die deutsche Bundesregierung auf asyl- und ausländerpolitische Maßnahmen, die die Menschenrechte Schutzsuchender massiv verletzen. 
Angesichts dieser politischen Entwicklungen ist die Rückbesinnung auf die Gründungsidee der Vereinten Nationen wegweisend,

„,unseren Glauben an die Grundrechte des Menschen, an Würde und Wert der menschlichen Persönlichkeit [...], erneut zu bekräftigen, Bedingungen zu schaffen, unter denen Gerechtigkeit und die Achtung vor den Verpflichtungen aus Verträgen und anderen Quellen des Völkerrechts gewahrt werden können“ (Charta der Vereinten Nationen, Präambel, UNRIC 2018: 3).

Als eines der zentralen Ziele formulieren die Vereinten Nationen die internationale Zusammenarbeit, um ,die Achtung vor den Menschenrechten und Grundfreiheiten für alle ohne Unterschied der Rasse, des Geschlechts, der Sprache oder der Religion zu fördern und zu festigen“" (ebd.).

An diese Grundüberzeugung gilt es anzuknüpfen und sich für die bedingungslose Umsetzung der Menschenrechte aller Menschen in Deutschland einzusetzen.

\section{Literatur}

Arendt, Hannah (1943/2017): Wir Flüchtlinge. Mit einem Essay von Thomas Meyer. Ditzingen: Reclam.

Bhabha, Jacqueline (2006): The child: What sort of human. In: PMLA (Modern Language Association of America), 121 (5), S. 1526-1535.

BMSFSJ/Unicef (2017): Mindeststandards zum Schutz von Kindern, Jugendlichen und Frauen in Flüchtlingsunterkünften. o.Ortsangabe.

Cocks, Alison (2006): The Ethical Maze - Finding an inclusive path towards gaining children's agreement to research participation. In: Childhood 13 (2), S. 247266.

Davis, James (2010): Introduction: Emotions in the Field: The Psychology and Anthropology of Fieldwork. In: Davis, James \& Spencer, Dimitrina (Hrsg.): Emotions in the Field: The Psychology and Anthropology of Fieldwork. California: Stanford University Press, S. 1-34.

Galtung, Johan (1969): Violence, Peace, and Peace Research. In: Journal of Peace Research 6 (3), S. 167-191, http://www.jstor.org/stable/422690 (Zugriff 30.11.2018).

Galtung, Johan (1990): Cultural Violence. In: Journal of Peace Research 27 (3), S. 291-305, http://links.jstor.org/sici?sici=0022-3433\%28199008\%2927\%3A3 \%3C291\%3ACV\%3E2.0.CO\%3B2-6 (Zugriff 30.11.2018).

ISTA (2018): Positionspapier, https://www.situationsansatz.de/ (Zugriff 30.11. 2018).

Initiative ASH (2016): Initiative Hochschullehrender zu Sozialer Arbeit in Gemeinschaftsunterkünften: Positionspapier: Soziale Arbeit mit Geflüchteten in 
Gemeinschaftsunterkünften. Professionelle Standards und sozialpolitische Basis, Berlin, http://www.fluechtlingssozialarbeit.de/ (Zugriff 27.8.2018).

Kaukko, Mervi \& Wernesjö, Ulrika (2016): Belonging and participation in liminality: Unaccompanied children in Finland and Sweden. In: Childhood, S. 114.

Koalitionsvertrag (2018): Ein neuer Aufbruch für Europa. Eine neue Dynamik für Deutschland. Ein neuer Zusammenhalt für unser Land. Koalitionsvertrag zwischen CDU, CSU und SPD, https://www.mdr.de/nachrichten/politik/inland/ download-koalitionsvertrag-quelle-spd-100.html (Zugriff 1.6.2018).

Maragel, Michel \& Manachi, Sandra (2018): The resilience of Syrian refugee children in Lebanon. In: Pace, Michelle \& Sen, Somdeep (Hrsg.): Syrian Refugee Children in the Middle East and Europe. Integrating the young and Exiled. London \& New York: Routledge, S. 32-45.

Niedrig, Heike \& Seukwa, Henri (2010): Die Ordnung des Diskurses in der Flüchtlingskonstruktion: eine postkoloniale Re-Lektüre. In: Diskurs Kindheits- und Jugendforschung 5 (2), S. 181-193.

Pupavac, Vanessa (2001): Misanthropy without borders: the international children's rights regime. In: Disasters 25 (2), S. 95-112.

Pupavac, Vanessa (2008): Refugee Advocacy, Traumatic Representations and Political disentchantment. In: Government and Opposition 43 (2), S. 270-292.

Save the children Deutschland (2018): „Unterbringungs-TÜV“. Zur Messung von Kinderrechten in Unterkünften für geflüchtete Menschen in Deutschland. Berlin

Sen, Amartya (2007): Children and Human Rights. IN: Indian Journal of Human Development 1 (2), S. 235-245.

Sen, Somdeep (2018): Writing the ,refugee crisis': proposals for activist research. In: Pace, Michelle \& Sen, Somdeep (Hrsg.): Syrian Refugee Children in the Middle East and Europe.Integrating the young and Exiled. London \& New York: Routledge, S. 101-112.

Senat Berlin (2016): Masterplan für Integration und Sicherheit, https://www.berlin.de/rbmskzl/aktuelles/politik-aktuell/2016/langfassung-masterplan-integration-und-sicherheit.pdf (Zugriff 15.6.2018).

Stebbins, Robert (1991): Do we ever leave the field? Notes on secondary fieldwork involvements. In: Shaffir, William \& Stebbins, Robert (Hrsg.): Experiencing Fieldwork: An Inside View of Qualitative Research. Newbury Park: Sage, S. 248-255.

UNRIC (2018) Charta der Vereinten Nationen und Statut des Internationalen Gerichtshofs, https://www.unric.org/html/german/pdf/charta.pdf (29.6.2018).

Weltgesundheitsorganisation (WHO) (2002): World Report on Violence and Health. Geneva.

Weltgesundheitsorganisation (WHO) (Hrsg.) (2003): Weltbericht Gewalt und Gesundheit. Zusammenfassung.

Zeit online (2018), Neue Härte. Asylpolitik. 24.1.2018, https://www.zeit.de/2018/ 05/asylpolitik-fluechtlinge-massenunterkuenfte-sondierungspapier (Zugriff 14.10.2018). 


\section{Angaben zu den Autorinnen}

Sarah Fichtner, Dr. phil. ist Sozial- und Kulturanthropologin. Sie arbeitet als freiberufliche, überwiegend ethnografisch vorgehende Forscherin, Filmemacherin und Beraterin (u. a. für Brot für die Welt, ChangeWriters e. V. und Unicef). $\mathrm{Zu}$ ihren Forschungsschwerpunkten gehören Schule, Bildungsreformen und Lehrerweiterbildungen in alternativen Lehrmethoden in West-, Zentralafrika und Deutschland sowie Flucht, Migration und das Alltagserleben von Kindern in Unterkünften für Geflüchtete in Deutschland.

Thi Huyen Trang Le arbeitet als Wissenschaftliche Mitarbeiterin im Institut für medizinische Soziologie an der Charité im Bereich Altersforschung. Sie ist staatlich anerkannte Kindheitswissenschaftlerin und hat den Studiengang Leitung, Bildung, Diversität mit dem Schwerpunkt Gesundheits- und Sozialmanagement (M. A.) an der Evangelischen Hochschule Berlin absolviert. Neben dem Studium engagierte sie sich in diversen Projekten, wie im „Netzwerk Integration“" und „MiMi - Mädchen mit Migrationshintergrund “. Zudem war sie in verschiedenen Forschungsprojekten zum Thema Schulgesundheit, Migration und frühe Kindheit involviert. Zu ihren Forschungsinteressen gehören empirische qualitative Methoden, Humor, Bildungsungleichheit, Flucht und Migration sowie Versorgung und Pflege am Lebensende.

Evelyn Schulz-Algie hat Sozial- und Kulturanthropologie sowie den Master in Childhood Studies and Children's Rights an der FU Berlin studiert. Sie ist qualifizierte Kinderschutzfachkraft und ausgebildete Koordinatorin im Internationalen Projektmanagement. In einem Kinderschutzzentrum in Ecuador arbeitete sie im Bereich der Sozialen Arbeit mit Kindern, Jugendlichen und Eltern. Als Programm- und Projektkoordinatorin in der Kindernothilfe e. V. begleitete sie kinderrechtsbasierte Projekte zu den Themen Bildung, Kinderschutz, Kinderarbeit und Gender in Haiti, Peru und Bolivien. Zudem leitete sie in der Servicestelle Jugendbeteiligung e. V. ein Projekt im Bereich Partizipation und Demokratieförderung in Berliner Schulen. Ihre Forschungsschwerpunkte sind Bildung, Migration und Flucht, Kinderschutz und Partizipation von Kindern sowie soziale Ungleichheit.

Penelope Scott, Dr. phil. ist Sozialwissenschaftlerin und Gastwissenschaftlerin am Institut für Soziologie, Ludwig-Maximilians-Universität München. Sie hat als qualitative Forscherin sowohl an der Universität London als auch am Wissenschaftszentrum Berlin und an der LMU München gearbeitet. Ihre Forschungsschwerpunkte sind Flucht, Migration und Gesundheit. Sie lehrte auch 
an Universitäten in Rio de Janiero und Berlin zu Themen Migration, Migration und Gesundheit, Soziologie von Gesundheit und Krankheit und zu empirischen qualitativen Methoden.

Hoa Mai Trần ist Kindheitspädagogin und Bildungs- und Erziehungswissenschaftlerin. Derzeit arbeitet sie als wissenschaftliche Mitarbeiterin zur digitalen Teilhabe von Kindern sowie als Lehrbeauftragte an der Hochschule Emden/Leer. Ihre praktischen Erfahrungen erstrecken sich von verschiedenen Krippen, Kitas, Vorschulen und Sekundarschulen über Armen- und Kinderheime bis zu therapeutischen Einrichtungen. Als politische Bildungsreferentin arbeitet sie zu den Themen Migration, Flucht/Asyl, Rassismus und Empowerment. Als Erwachsenenbildnerin sind ihre Arbeitsschwerpunkte vorurteilsbewusste Bildung und Erziehung, Diversität/Heterogenität und inklusive pädagogische Praxis im Elementarbereich. Ihre Expertise in der Wissenschaft liegt in der quantitativen sowie qualitativen Forschung mit Schwerpunkt Kindheitsforschung und sozialer Ungleichheitsforschung. Sie engagiert sich darüber hinaus im Projektfonds für kulturelle Bildung und als Projektleitung in einem kollaborativen mehrsprachigen Kinderbuchprojekt.

Anne Wihstutz, Dr. phil. ist Professorin für Soziologie an der Evangelischen Hochschule Berlin, sie hat die wissenschaftliche Leitung des Projekts „Alltagserleben von geflüchteten Kindern bis 6 Jahren in Gemeinschafts- und Notunterkünften“" (2016-2017), angesiedelt am INIB e.V. und dem ISTA an der Internationalen Akademie in Berlin, inne. Ihre Lehrgebiete umfassen u. a. die Soziologie der Kindheit, Ungleichheitstheorien, internationale Kindheiten. Sie forscht u. a. zu frühkindlicher Bildung und Partizipation in Deutschland, Arbeit von Kindern, Kinderrechten, Flucht und Migration, Gender. Nach ihrem Studium der Sozialpädagogik/Sozialarbeit an der Alice-Salomon Hochschule Berlin (ASH) arbeitete sie mehrere Jahre mit Jugendlichen, Eltern und Lehrer*innen in Chile und Tansania sowie in Berlin und studierte im Anschluss an der TU Berlin Soziologie und Erziehungswissenschaft. Sie promovierte an der TU Berlin zur Bedeutung von Arbeit für Kinder. Als Postdoc an der MartinLuther-Universität Halle (Saale) forschte und lehrte sie zum Themengebiet gesellschaftlicher Sorgearbeit. Sie hat zahlreiche Publikationen veröffentlicht zu Haus- und Sorgearbeit, Citizenship und Agency von Kindern. 


\section{Zwischen Sandkasten und Abschiebung}

Basierend auf einer mehrmonatigen ethnografischen Feldforschung beschreiben die Beiträge das Leben junger Kinder in Unterkünften für geflüchtete Menschen. Insbesondere die räumlich-materielle Ausstattung der Unterkünfte wird in ihrer sozialen und emotionalen Bedeutung für die Kinder und ihre Eltern erfahrbar und vor dem Hintergrund universeller Kinderrechte diskutiert. Das Buch nimmt eine kritische Einschätzung der Verhältnisse vor und richtet Empfehlungen an Politik, Praxis und Wissenschaft. Es eignet sich als Grundlagentext für den Einsatz in der Aus- und Weiterbildung (sozial-)pädagogischer Fachkräfte.

„Nach meinem Eindruck ist ein Buch herausgekommen, in dem die Sichtweisen, Gedanken, Sorgen und Wünsche geflüchteter, in Sammelunterkünften lebender Kinder auflebendige und nachvollziehbare Weise zum Ausdruck kommen."

Manfred Liebel

Die Herausgeberin:

Prof. Dr Anne Wihstutz,

Professorin, Evangelische Hochschule Berlin

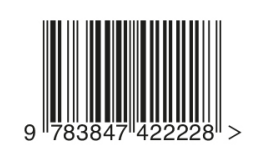

www.budrich-verlag.de 